\title{
Targeting Foam Cell Formation in Atherosclerosis: Therapeutic Potential of Natural Products
}

\author{
Dongdong Wang, Yang Yang, Yingnan Lei, Nikolay T. Tzvetkov, Xingde Liu, Andy Wai Kan Yeung, Suowen Xu, and Atanas G. Atanasov \\ The Second Affiliated Hospital of Guizhou University of Traditional Chinese Medicine, Guiyang, China (D.W., X.L.); Department of \\ Molecular Biology, Institute of Genetics and Animal Breeding of the Polish Academy of Sciences, Jastrzębiec, Poland (D.W., Y.Y., Y.L., \\ A.G.A.); Department of Pharmacognosy, University of Vienna, Vienna, Austria (A.G.A.); Institute of Clinical Chemistry, University Hospital \\ Zurich, Schlieren, Switzerland (D.W.); Institute of Molecular Biology "Roumen Tsanev," Department of Biochemical Pharmacology and \\ Drug Design, Bulgarian Academy of Sciences, Sofia, Bulgaria (N.T.T.); Pharmaceutical Institute, University of Bonn, Bonn, Germany \\ (N.T.T.); Aab Cardiovascular Research Institute, Department of Medicine, University of Rochester, Rochester, New York (S.X.); Oral and \\ Maxillofacial Radiology, Applied Oral Sciences and Community Dental Care, Faculty of Dentistry, The University of Hong Kong, Hong \\ Kong, China (A.W.K.Y.); and Institute of Neurobiology, Bulgarian Academy of Sciences, Sofia, Bulgaria (A.G.A.)
}

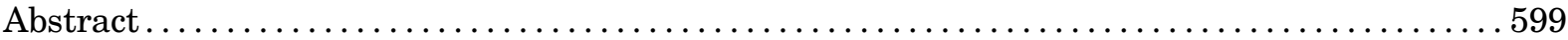

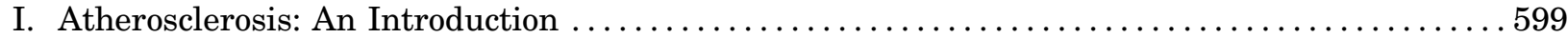

II. Foam Cell Formation as a Hallmark of Atherosclerosis . . . . . . . . . . . . . . . . . . . . 600

A. Cholesterol Uptake ................................................... 600

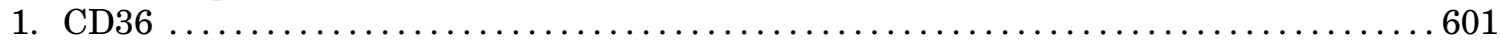

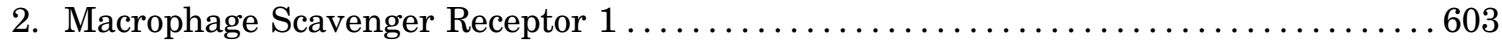

3. Lectin-like Oxidized Low-density Lipoprotein Receptor-1 ................... 604

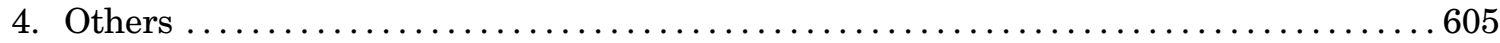

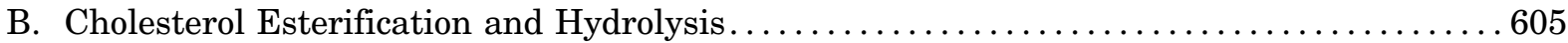

1. Acetyl-CoA Acetyltransferases 1 and Acetyl-CoA Acetyltransferases $2 \ldots \ldots \ldots \ldots 605$

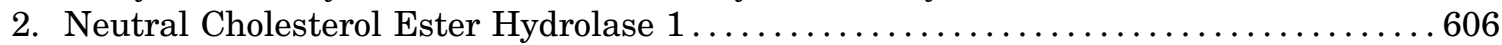

3. Lysosomal Acid Lipase (Lipase A) and Hormone-sensitive Lipase 1 (Lipase E) . . . . 606

C. Macrophage Cholesterol Efflux ...................................... 607

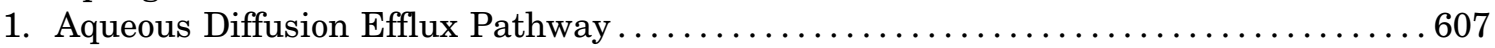

2. Transporter-Dependent Cholesterol Efflux Pathway $\ldots \ldots \ldots \ldots \ldots \ldots \ldots \ldots \ldots \ldots \ldots 67$

a. ATP-binding Cassette Transporter A1 ............................. 607

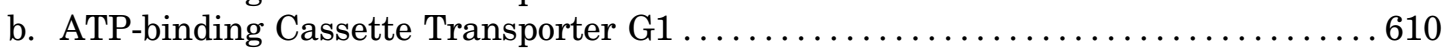

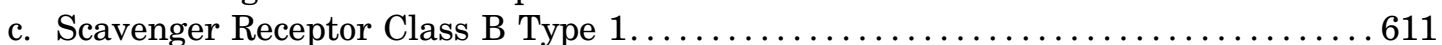

3. Acceptors that Mediate Macrophage Cholesterol Efflux .....................612

a. Apolipoprotein A-1 ........................................... 612

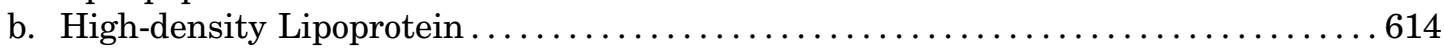

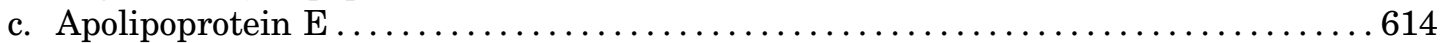

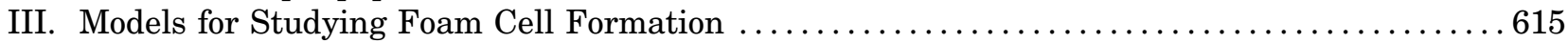

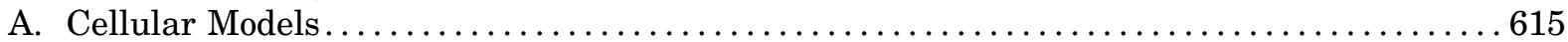

1. Models for Studying Cholesterol Uptake ..............................6 615

2. Models for Studying Cholesterol Efflux. .............................6 616

a. Loading cells with labeled lipids to form foam cells $\ldots \ldots \ldots \ldots \ldots \ldots \ldots \ldots \ldots 6$

b. Inducing cholesterol efflux to extracellular acceptors and quantification . . . . . . 617

B. Animal Models for Studying Foam Cell Formation........................ 618

Address correspondence to: Dr. Atanas G. Atanasov, Department of Molecular Biology, Institute of Genetics and Animal Breeding of the Polish Academy of Sciences, ul. Postepu 36A, Jastrzebiec, 05-552 Magdalenka, Poland. E-mail: atanas.atanasov@univie.ac.at and a.atanasov.mailbox@gmail.com; or Dr. Suowen Xu, Aab Cardiovascular Research Institute, Department of Medicine, University of Rochester, Rochester, NY, 14623. E-mail: Suowen_Xu@URMC.Rochester.edu and suowen.xu@gmail.com

This work was supported by the Cultivation Project for Clinical Medicine of the Integrated Traditional Chinese and Western Medicine and Cultivation Project for Education team of internal medicine of the integrated traditional Chinese and western medicine in the first-term subjects, with special support from the first-class universities in Guizhou province [Qin Jiao Gao Fa No. 2017-158], Polish KNOW (Leading National Research Centre) Scientific Consortium "Healthy Animal-Safe Food" [decision of Ministry of Science and Higher Education No. 05-1/KNOW2/2015], and the Peter und Traudl Engelhorn Foundation for the promotion of Life Sciences. S.X. is supported by American Heart Association Career Development Award [18CDA34110359]. https://doi.org/10.1124/pr.118.017178. 
IV. Natural Products Targeting Foam Cell Formation in Atherosclerosis..................619

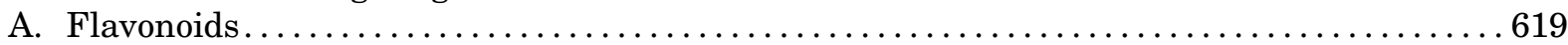

1. Alpinetin .................................................. 619

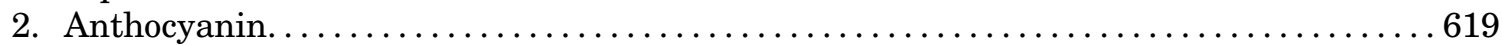

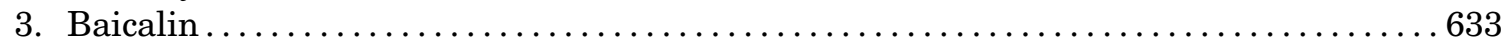

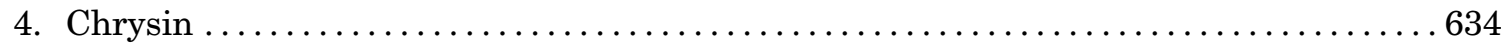

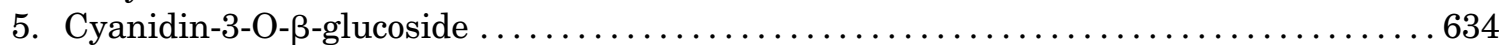

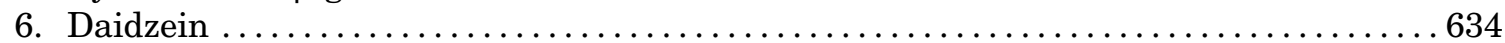

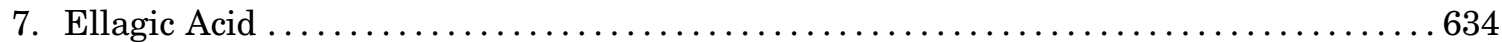

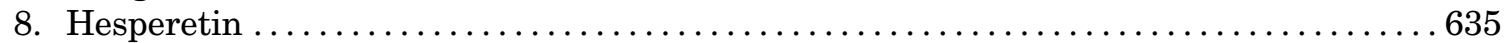

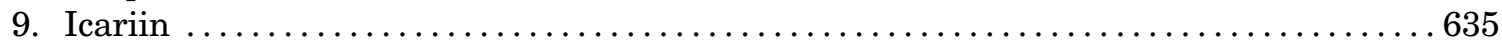

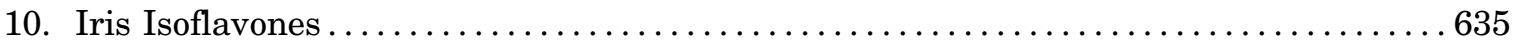

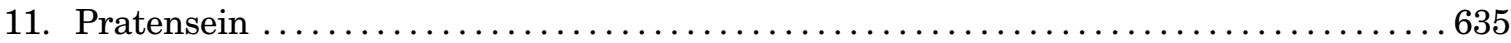

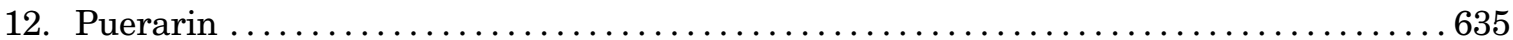

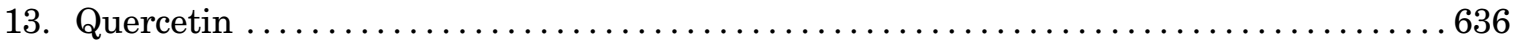

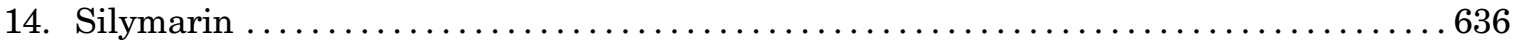

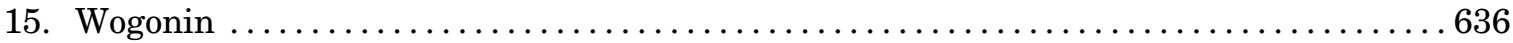

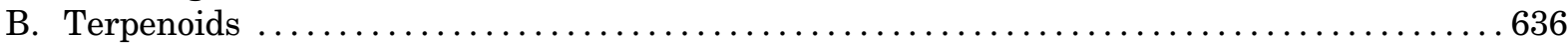

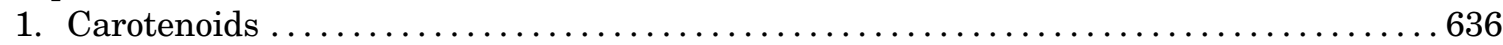

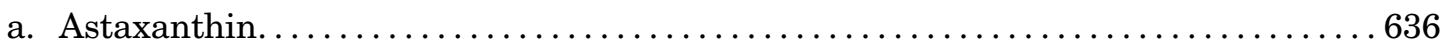

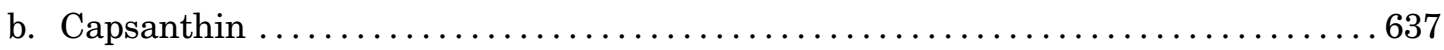

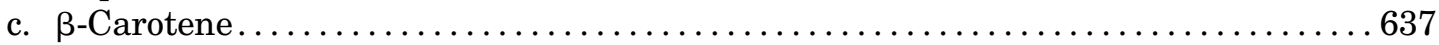

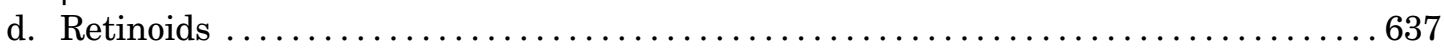

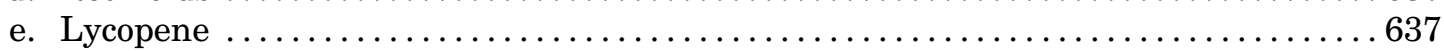

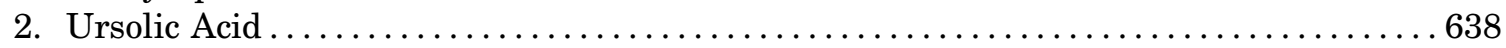

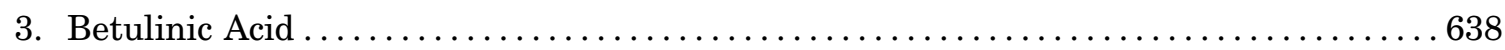

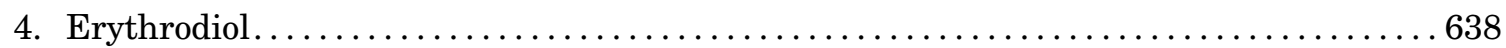

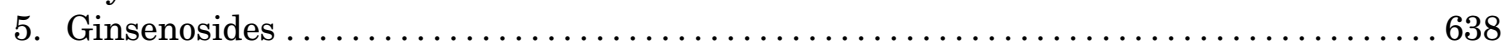

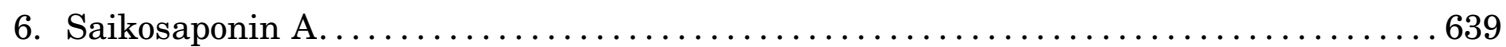

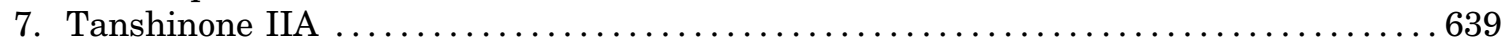

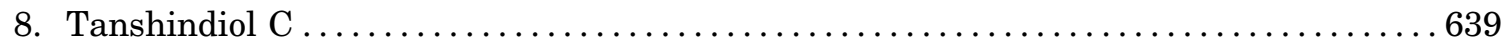

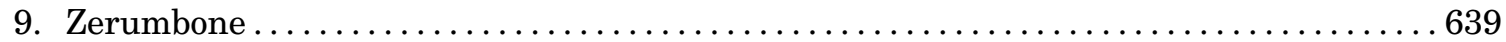

ABBREVIATIONS: ABCA1, ATP-binding cassette transporter A1; ABCG1, ATP-binding cassette transporter G1; AC, anthocyanins; ACAT, acetyl-CoA acetyltransferases; acLDL, acetylated LDL; AMPK, AMP-activated protein kinase; AP-1, activating protein-1; ApoA-1, apolipoprotein A-1; ApoE, apolipoprotein E; APS, astragalus polysaccharides; ATRA, all-trans retinoic acid; BET, extra terminal bromodomains; BMDMs, bone marrow-derived macrophages; BODIPY, dipyrromethene boron difluoride; $\mathrm{C} 3 \mathrm{G}$, cyanidin-3-O-glucoside; CAD, coronary artery disease; CE, cholesterol esters; CETP, cholesteryl ester transfer protein; 9-cis- $\beta$ c, 9-cis- $\beta$-carotene; CLA-1, clarinet 1; CVD, cardiovascular disease; CXCL16, C-X-C motif chemokine ligand 16; DHA, docosahexaenoic acid; DiI, 1,1'-dioctadecyl-3,3,3',3'-tetra-methylindocyanide percholorate; EGCG, epigallocatechin gallate; eNOS, endothelial NO synthase; EPA, eicosapentaenoic acid; ER, endoplasmic reticulum; FC, free cholesterol; F-Ch, fluorophore Pennsylvania green/ $N$-alkyl-3b-cholesterylamine-derived molecular probe; GLP-1, glucagon-like peptide-1; HDL, high-density lipoprotein; HDL-C, HDL-cholesterol; HFD, high fat diet; HMDM, human monocyte-derived macrophages; 12-HODE, 13hydroxyoctadecadienoic acid; HOTAIR, homeobox protein transcription antisense RNA; hs-CRP, high sensitivity C-reactive protein; HSL, hormone-sensitive lipase; HUVEC, human umbilical vein endothelial cells; ICA, icariin; ICAM1, intercellular adhesion molecule 1; IL, interleukin; JNK, c-Jun N-terminal kinases; LAL, lysosomal acid lipase; LCAT, lecithin cholesterol acyltransferase; LD, lipid droplets; LDL, low-density lipoprotein; LDL-C, LDL-cholesterol; LDLR, LDL receptor; lncRNA, long noncoding RNA; LOX-1, lectin-like oxidized LDL receptor 1; LPS, lipopolysaccharide; LXR, liver X receptor; MALAT1, metastasis associated lung adenocarcinoma transcript 1; MAPK, mitogenactivated protein kinase; MARCO, macrophage receptor with collagenous structure; miRNA, microRNAs; MPM, mouse peritoneal macrophages; NA, nicotinic acid; NCEH, neutral CE hydrolases; ncRNA, noncoding RNAs; Neat1, nuclear paraspeckle assembly transcript 1; NFIA, nuclear factor IA; NF- $\kappa \mathrm{B}$, nuclear transcription factor- $\kappa \mathrm{B}$; NHERF, PDZK1/ $\mathrm{Na}^{+} / \mathrm{H}^{+}$exchanger regulatory factor; NO, nitric oxide; NPC1, Niemann-Pick disease, type C1; NPC1L1, NPC1 like intracellular cholesterol transporter 1; Nrf2, nuclear erythroid-related factor 2; oxLDL, oxidized LDL; PCA, protocatechuic acid; PCSK9, proprotein convertase subtilisin/kexin type 9; PEA, pomegranate ellagic acid; PGG, 1,2,3,4,6penta-O-galloyl- $\beta$-D-glucose; PI3K, phosphoinositide 3-kinase; PKA, protein kinase A; PKC, protein kinase C; PLPEs, phellinus linteus polysaccharide extracts; PNS, panax notoginseng saponins; PON-1, paraoxonase-1; PPAR, peroxisome proliferator-activated receptor; PXR, pregnane X receptor; RCT, reverse cholesterol transport; rHDL, reconstituted HDL; ROS, reactive oxygen species; RXR, retinoid X receptor; Sirt1, sirtuin 1; SR, scavenger receptor; SR-BI, scavenger receptor class B type 1; SREBP, sterol regulatory element-binding proteins; STAT, signal transducer and activator of transcription; TC, total cholesterol; TG, triglycerides; TGF- $\beta$, transforming growth factor- $\beta$; TLR, toll-like receptor; TMAO, trimethylamine $N$-oxide; TMD, transmembrane domains; TNF- $\alpha$, tumor necrosis factor- $\alpha$; trans- $\beta$ c, trans- $\beta$-carotene; UA, ursolic acid; UTR, untranslated regions; VCAM-1, vascular cell adhesion molecule 1; VSMC, vascular smooth muscle cells. 
C. Phenolic Compounds ................................................. 639

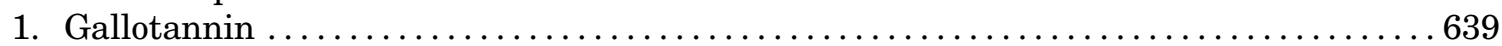

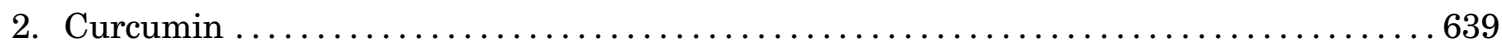

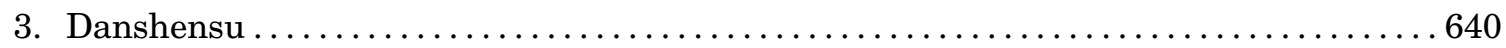

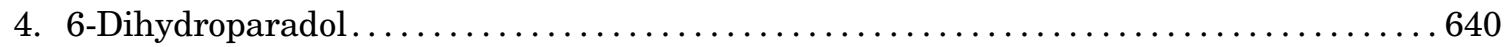

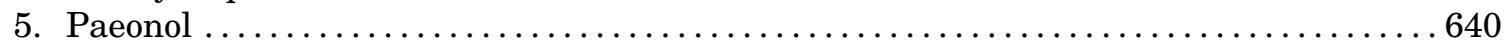

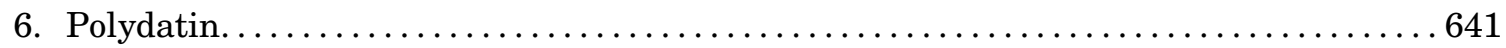

7. Protocatechuic Acid............................................... 641

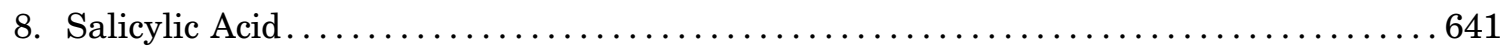

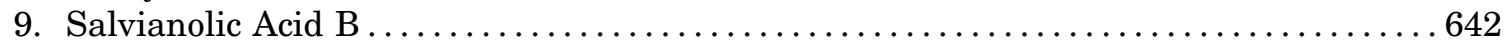

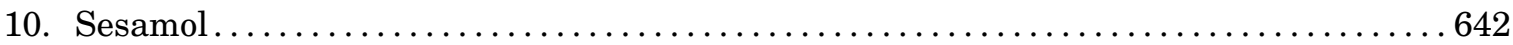

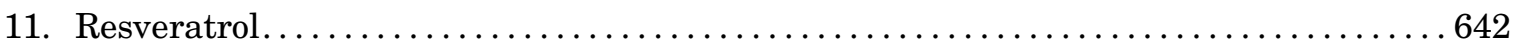

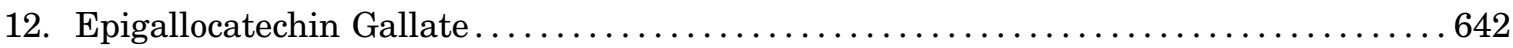

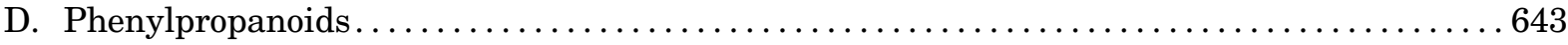

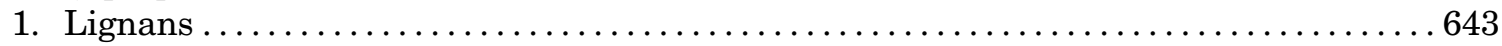

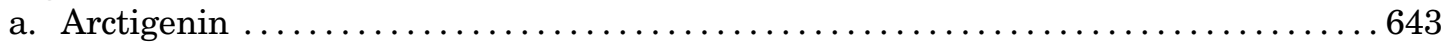

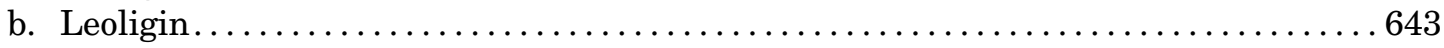

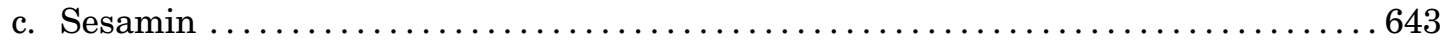

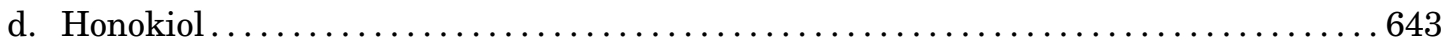

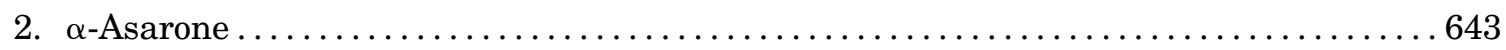

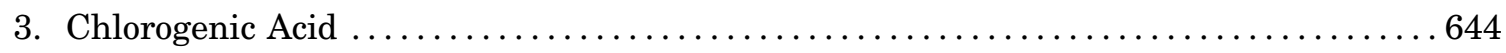

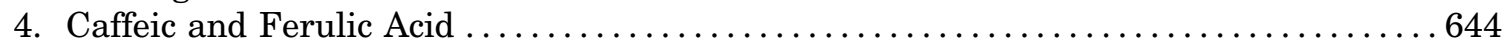

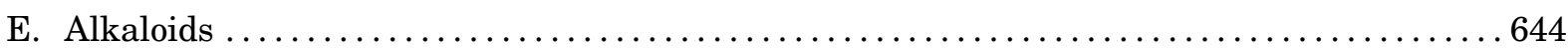

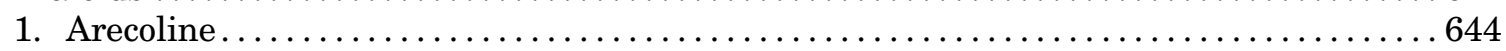

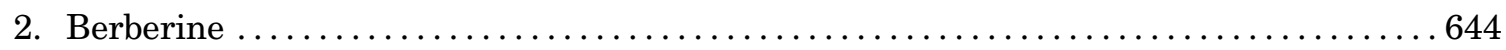

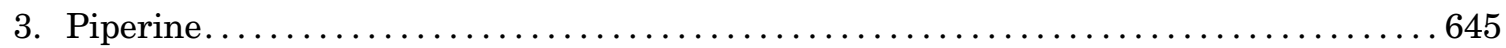

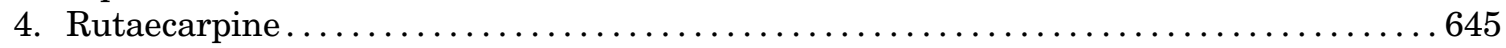

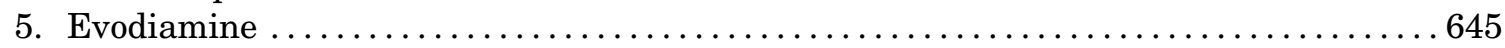

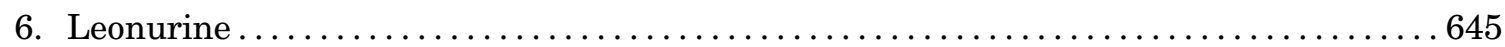

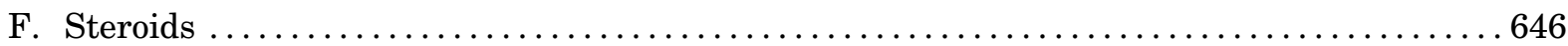

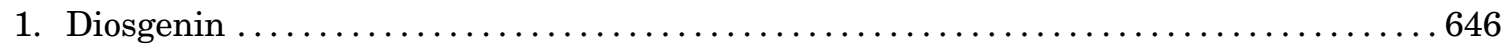

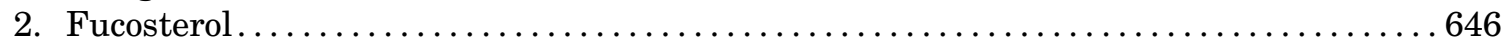

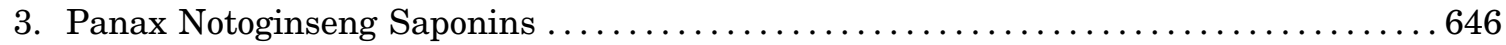

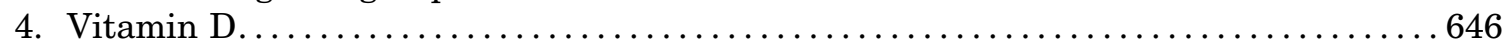

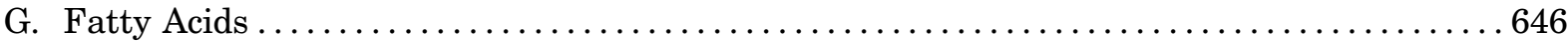

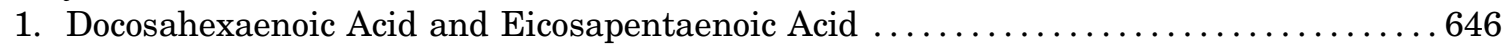

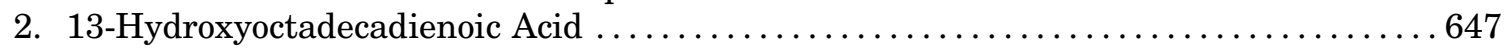

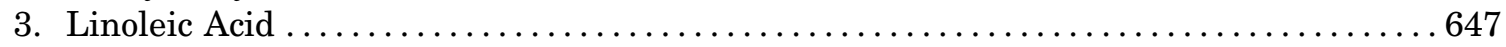

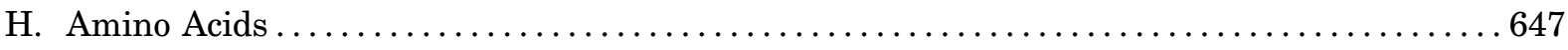

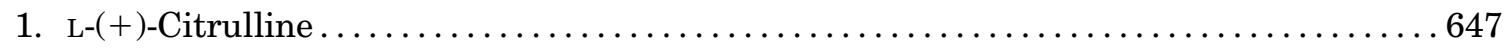

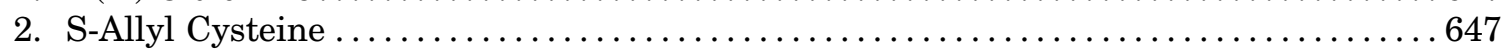

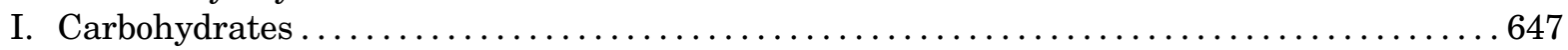

1. Phellinus Linteus Polysaccharides .............................. 647

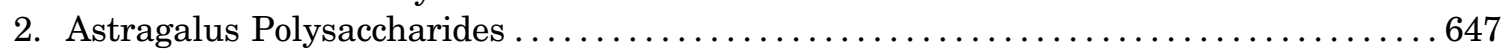

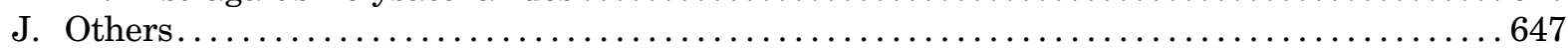

1. Organosulfur Compounds: Allicin ................................. 647

2. Pyranone Derivatives: Asperlin ................................6648

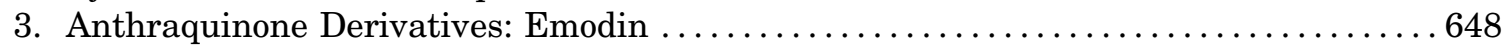

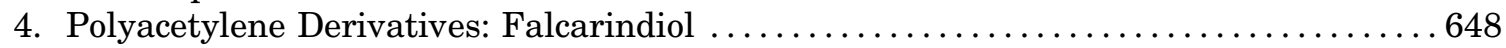

5. Marine Natural Products ....................................... 648

V. Clinically Used Drugs that Influence Foam Cell Formation ........................6 648

A. Approved Drugs ................................................... 648

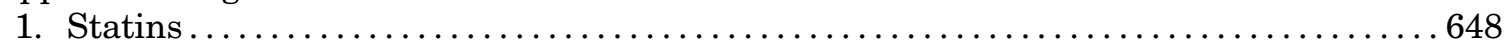

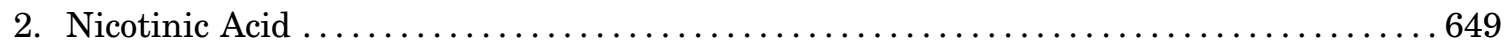

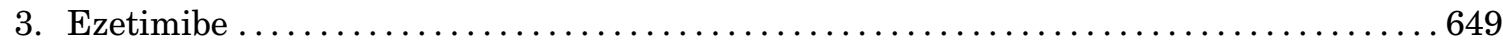

4. Proprotein Convertase Subtilisin/Kexin Type 9Antibodies ...................6 649 
B. Drugs in Clinical Trials ............................................. 649

1. Glucagon-like Peptide-1 Receptor Agonists ............................ 649

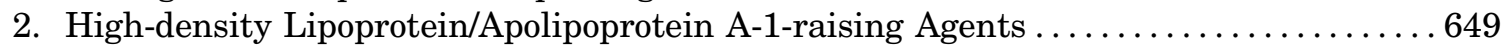

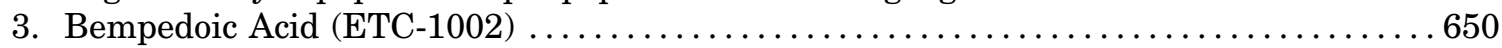

VI. The Role of Inhibition of Foam Cell Formation in Antiatherosclerosis Therapy ..........6651

VII. Concluding Remarks and Future Perspectives ............................ 652

References........................................................... 654

\begin{abstract}
Foam cell formation and further accumulation in the subendothelial space of the vascular wall is a hallmark of atherosclerotic lesions. Targeting foam cell formation in the atherosclerotic lesions can be a promising approach to treat and prevent atherosclerosis. The formation of foam cells is determined by the balanced effects of three major interrelated biologic processes, including lipid uptake, cholesterol esterification, and cholesterol efflux. Natural products are a promising source for new lead structures. Multiple natural products and pharmaceutical agents can
\end{abstract}

inhibit foam cell formation and thus exhibit antiatherosclerotic capacity by suppressing lipid uptake, cholesterol esterification, and/or promoting cholesterol ester hydrolysis and cholesterol efflux. This review summarizes recent findings on these three biologic processes and natural products with demonstrated potential to target such processes. Discussed also are potential future directions for studying the mechanisms of foam cell formation and the development of foam cell-targeted therapeutic strategies.

\section{Atherosclerosis: An Introduction}

Atherosclerosis is a chronic inflammatory (Ross, 1999; Kasikara et al., 2018), immune (Hansson and Hermansson, 2011), and epigenetic (Xu et al., 2018, 2019) disease characterized by low-density lipoprotein (LDL) retention and defective resolution of vascular inflammation in the vessel wall. It is the major cause of acute cardiovascular events, such as unstable angina pectoris, myocardial infarction, ischemic stroke, and sudden cardiovascular death (Tabas et al., 2015). The pathobiology of atherosclerosis is very complex and involves multiple cell types, such as endothelial cells, monocytes, macrophages, vascular smooth muscle cells (VSMCs), T cells, B cells, mast cells, and dendritic cells (Tabas et al., 2015). Atherosclerosis preferentially develops at arterial branching points and ascending aortic arch, where local disturbance of blood flow occurs. In contrast, regions of high laminar shear stress, such as thoracic aorta, are protected against atherosclerosis (Davies, 2009; Chiu and Chien, 2011; Rezvan et al., 2011; Abe and Berk, 2014; Baeyens et al., 2016; Niu et al., 2019). Shear stress can also regulate atherosclerosis by causing the breach of the intimal layer that leads to increase of inflammation, leukocyte adhesion, and the occurrence of late stage atherothrombotic events (Franck et al., 2019). Therefore, the integrity of the vascular endothelium is critical for maintaining endothelial function, such as regulation of vascular tone, antioxidative response, and antithrombotic effects (Vanhoutte et al., 2017; Nafisa et al., 2018). It has been well established that passive movement of LDL across the compromised endothelial layer instigates atherosclerosis. A recent study has shown that scavenger receptor class B type I (SR-BI)-mediated endothelial LDL transcytosis also promotes LDL entry into the artery wall and promotes atherosclerosis development, raising a new therapeutic target in combating atherosclerosis (Huang et al., 2019). Dysfunctional properties of endothelial cell cause endothelial dysfunction, which leads to the development of atherosclerosis (Vanhoutte et al., 2017; Nafisa et al., 2018). Under such circumstances, circulating LDLs penetrate into the injured vascular endothelium and accumulate in the subendothelial space, where LDLs are prone to various atherogenic modifications, including oxidation, glycation, enzymatic modification, and carbamylation (Alique et al., 2015). Among these modifications, oxidation is the most common form of atherogenic modification (Quinn et al., 1987). It occurs during increased oxidative stress via cellular sources and enzymatic routes [such as nicotinamide adenine dinucleotide phosphate (NADPH) oxidases, lipoxygenases, and myeloperoxidases] (Quinn et al., 1987). These types of modified LDL mimic damageand pathogen-associated molecular patterns, thus serving as the primary sources of cholesterol accumulation and chronic inflammation in atherosclerotic plaques (Tabas et al., 2015). In addition, these modified LDLs can cause oxidative stress, inflammation, NLRP3 inflammasome activation, and immune response, thus amplifying the vicious cycle of atherogenic cellular events (Steinberg and Witztum, 2010; Wang et al., 2017h). In addition to modified LDL, interleukin 1 (IL-1) (IL- $1 \alpha$ and IL- $1 \beta)$ also drives vascular inflammation, with IL- $1 \alpha$ mainly being involved in arterial remodeling during the early phase of atherosclerosis, whereas IL- $1 \beta$ drives inflammation and atheroprogression to advanced state of atherosclerosis (Vromman et al., 2019). The recent CANTOS clinical trial has shown that anti-inflammatory effect with canakinumab (a monoclonal antibody targeting IL-1 $\beta$ ) significantly reduces the rate of recurrent cardiovascular events in patients with atherosclerotic diseases without reducing the lipid levels (Ridker et al., 2017). IL-1 $\beta$, together with other proatherogenic stimuli 
such as modified LDL, can promote endothelial activation and persistent inflammatory responses lead to the proliferation, migration, phenotypic switching of VSMCs, and lipid accumulation in VSMCs [i.e., VSMC-derived foam cell formation (Allahverdian et al., 2012)]. Cholesterol loading (by incubation with cyclodextrin-cholesterol complexes) leads to an inhibited expression of the VSMC marker genes and increased expression of proinflammatory and macrophage marker genes (Rong et al., 2003; Vengrenyuk et al., 2015). These VSMCs could substantially contribute to pooled population of foam cells (Allahverdian et al., 2014). Lineage tracing experiments have shown that VSMC can form foam-like cells, and transcription factor Kruppel-like factor 4 and octamer-binding transcription factor 4 can differentially regulate VSMC-derived foam cell formation in mice (Shankman et al., 2015; Cherepanova et al., 2016). The existence of VSMC-derived foam cells in mouse and human atherosclerotic plaques significantly expand our understanding of the contribution of foam cells and the lipid-deposition theory of atherosclerosis (Allahverdian et al., 2014; Wang et al., 2019b).

Macrophages are heterogeneous immune and metabolic cells that play important roles in the initiation, growth, and ultimate rupture of atherosclerotic plaques (Koelwyn et al., 2018) and serve as an elusive therapeutic target of treating atherosclerosis and designing of novel antiatherosclerotic drugs (Santamarina-Fojo et al., 2000; Tiwari et al., 2008). It has been well recognized that monocytes in the bloodstream are recruited to the atherosclerotic lesion site after endothelial injury and increased vascular permeability. These recruited monocytes then differentiated into mature macrophages (in local prodifferentiation microenvironment), which avidly ingest modified LDL and accumulate lipids, thus giving rise to the formation of "foam cells" stuffed with lipid droplets (LD) (Tabas et al., 2015). Lesional macrophages can also proliferate, self-renew, and accumulate in advanced atherosclerotic lesions in mice. This mechanism represents an important pathway of macrophage build-up and probable macrophage-derived foam cell formation via macrophage replication, in addition to monocyte influx/infiltration (Robbins et al., 2013). The lipid in foam cells (derived from macrophages and VSMCs) represents the major source of plaque lipid content and drives the development of fatty streak and atherosclerotic plaque formation. Macrophages have migratory potential, however, under stimulation with oxidized LDL (oxLDL) the migratory capability has shown to be reduced, leading to macrophages trapping in the arterial wall and atheroprogression (Park et al., 2009b). Macrophages regulate inplaque inflammatory response and oxidative stress by secreting various cytokines and chemokines, as well as generating reactive oxygen species (ROS). In advanced plaques, the death of macrophages drives the enlarged formation of necrotic core. Normally, the necrotic core within the plaque is covered by a "protective" barrier-the fibrous cap, which serves as an "insulator" between platelets in circulating blood and prothrombotic substances within the lesion (Tabas et al., 2015). The thickness of the fibrous cap is determined by the balanced effects of collagen synthesis by VSMCs and collagen degradation by matrix metalloproteinase 2/9 (MMP-2/9). However, when the necrotic core size is increased (such as under conditions of increased primary necrosis, VSMC apoptosis, defective efferocytosis, and defective inflammation resolution by specialized proresolving mediators) and the fibrous cap gets thinner, the plaques are susceptible to rupture (Tabas et al., 2015; Bennett et al., 2016). The rupture of plaques leads to a vessel occlusion, an arterial thrombosis, and the occurrence of acute cardiovascular events (Arbab-Zadeh et al., 2012; Libby, 2013).

\section{Foam Cell Formation as a Hallmark of Atherosclerosis}

In the setting of atherosclerosis, lipid uptake and cholesterol esterification are increased, while cholesterol efflux is insufficient. The final outcome is excessive accumulation of cholesterol esters (CE) in macrophages to form foam cells (Yu et al., 2013; Maguire et al., 2019). Therefore, the formation of foam cells is determined by the balanced effects of three major interrelated biologic processes, including lipid uptake, cholesterol esterification, and cholesterol efflux. Each process is regulated by multiple transcription factors, including receptors, enzymes, and transporters, all of which work in concert to regulate lipid homeostasis (Yu et al., 2013; Maguire et al., 2019). Yearly publication counts on "foam cell" have been increasing dramatically (Fig. 1). Multiple natural products and pharmaceutical agents can inhibit foam cell formation and atherosclerosis by affecting one or more of the involved biologic processes. The gene expression of scavenger receptors (SRs) and cholesterol transporters can be regulated through transcriptional, posttranscriptional, translational, and epigenetic mechanisms [such as microRNAs (miRNA) and long noncoding RNAs (lncRNAs)]. Also, macrophage-derived foam cell formation is influenced by multiple cytokines and chemokines (McLaren et al., 2011a). Therefore, targeting the outlined major pathways (uptake, esterification, and efflux) could be effective therapeutic strategies to combat atherosclerosis (Fig. 2).

\section{A. Cholesterol Uptake}

Cholesterol uptake is a biologic process by which modified LDLs (such as oxLDL) are taken up by macrophages and VSMCs via SR-mediated pathways, as well as phagocytosis (Schrijvers et al., 2007) and pinocytosis (via receptor-independent endocytic pathway) (Kruth, 2011; Moore et al., 2013). Among these mechanisms, the SR-mediated uptake is the major pathway of cholesterol uptake. The primary functions of SRs are to clear invading pathogens and apoptotic cells as well as modified 


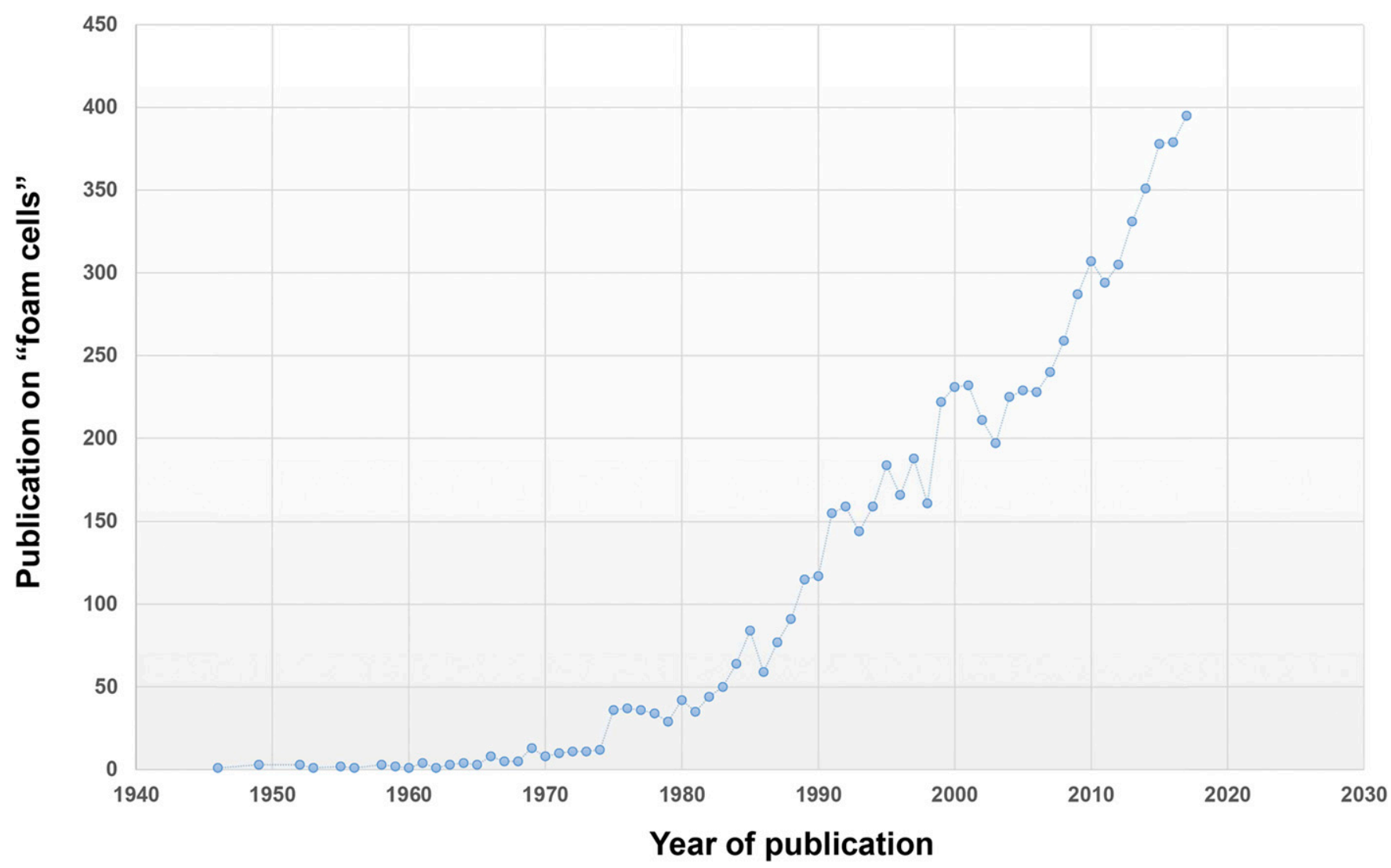

Fig. 1. Yearly publication counts on foam cells for the period 1946-2017. PubMed search was performed on Sep 11, 2018 using the subject term "foam cells."

lipids. Seminal findings from the Brown and Goldstein laboratories in 1970s (Brown et al., 1979; Goldstein et al., 1979) showed that the uptake of modified LDLs by macrophage "scavenger receptors" is faster than the uptake of native LDL. Since then, burgeoning studies have identified various SRs and elucidated the contribution of SRs to atherosclerosis development in diverse mouse models. Macrophages express several SRs that can bind, internalize, and degrade modified LDL, such as SR-A, CD36, and lectin-like oxLDL receptor-1 (LOX-1) (Xu et al., 2013c). Other SRs include macrosialin/CD68, SR expressed in endothelial cells, macrophage receptor with collagenous structure (MARCO), and C-X-C motif chemokine ligand 16 (CXCL16, also known as SR-PSOX) (Greaves and Gordon, 2005). The different SRs have different binding affinities and preference for various forms of modified LDL. Quantitative analysis in SR-A and CD36 knockout macrophages have shown that SR$\mathrm{A}$ and CD36 account for $75 \%-90 \%$ of oxLDL internalization by macrophages (Kunjathoor et al., 2002; Rahaman et al., 2006). Experiments in LOX-1-deficient macrophages indicated that LOX-1 accounts for 5\%-10\% of oxLDL uptake by murine macrophages under basal conditions, but in lysophosphatidylcholine-treated macrophages, the contribution of LOX-1 to macrophage oxLDL uptake and lipid accumulation is increased by more than 40\% (Schaeffer et al., 2009). Thus, CD36, SR-A, and LOX-1 are the major SRs responsible for the binding and subsequent uptake of modified LDL by macrophages (Kunjathoor et al., 2002). A detailed description of functions of all SRs in health and disease was reviewed elsewhere (Zani et al., 2015).

1. CD36. CD36 (also known as fatty acid translocase, FAT) belongs to class B of the SR family, representing $88 \mathrm{kDa}$ heavily glycosylated transmembrane platelet glycoprotein III b/IV (Yu et al., 2013). It was first identified in 1993 as a macrophage receptor that binds (with high affinity), internalizes, and degrades oxLDL (Endemann et al., 1993). CD36 has an extracellular domain, two cytoplasmic domains, and two transmembrane domains. CD36 is ubiquitously expressed in multiple cell types, including monocytes/macrophages, endothelial cells, platelets, and many others (Yu et al., 2013). In addition to regulating modified LDL binding, CD36 has other functions as well, such as involvement in inflammatory processes, lipid metabolism, fatty acid transport, and immunity (Febbraio et al., 2001). Another important function of CD36 is to modulate the migration of macrophages upon oxLDL stimulation, which may contribute to macrophage trapping/retention in arterial lesions (Park, 2014). CD36 can also be released to the circulation in patients with atherosclerosis, which leads to the formation of soluble CD36 (Handberg et al., 2008). Thus, soluble CD36 is a promising 


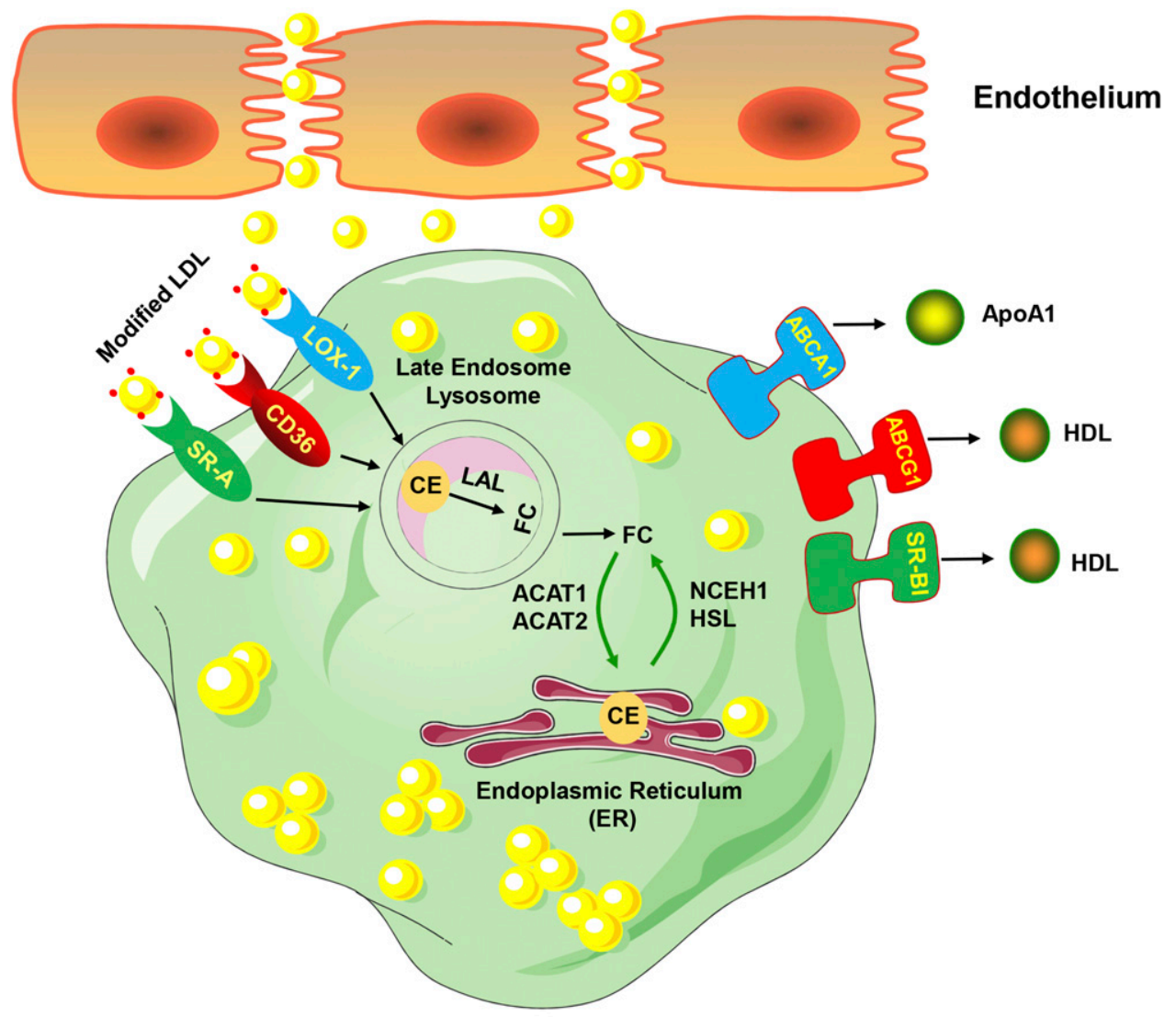

Macrophage-derived foam cell

Fig. 2. Therapeutic targets in macrophage-derived foam cell formation. After endothelial injury, LDL penetrates endothelial monolayer, then LDL is modified to form atherogenically modified LDL (such as oxidized LDL). The modified LDL is taken up by multiple scavenger receptors (SRs, such as CD36, SR-A, and LOX-1) on the cell membrane of differentiated macrophages. Then, LDL is delivered to late endosomes/lysosomes, where lysosomal acid lipase (LAL) degrades cholesteryl esters (CE) from the LDL particles to generate free cholesterol (FC) and free fatty acids. The FC can be reesterified by acyl-coenzyme A: cholesterol acyltransferase-1 and -2 (ACAT1 and ACAT2) in the endoplasmic reticulum (ER), thus contributing to CE accumulation and lipid droplet formation. $\mathrm{CE}$ can be hydrolyzed by neutral cholesteryl ester hydrolases (NCEHs), such as NCEH1 and hormonesensitive lipase (HSL) to liberate FC, which can be reverse transported outside of macrophages by cholesterol efflux transporters, ATP-binding cassette A1 (ABCA1, ApoA-1 as the acceptor) and G1 (ABCG1, HDL as the acceptor), as well as SR-B1 (HDL as the acceptor). Under physiologic conditions, cholesterol homeostasis is fine-tuned by a balance of "input" and "output" of lipids. Under pathologic conditions (such as atherosclerosis), the balance is disrupted, leading to foam cell formation. Therefore, there are three major strategies to reduce foam cell formation, i.e., by 1 ) reducing the SR-mediated lipid uptake, 2) reducing the ACAT-mediated cholesterol esterification, and 3) promoting the transporter-mediated cholesterol efflux. Other strategies to inhibit foam cell formation include the decrease of LDL modification and inhibition of monocyte to macrophage differentiation.

biomarker of plaque instability and symptomatic carotid atherosclerosis, as well as associated with carotid intima-media thickness (Handberg et al., 2008; Jiang et al., 2017b).

The expression of CD36 in macrophages is upregulated by several proatherogenic stimuli, such as oxLDL (Endemann et al., 1993), palmitate (Kim et al., 2017), and dysfunctional HDL from coronary artery disease (CAD) patients (Sini et al., 2017). The CD36 gene transcription is regulated by peroxisome proliferator-activated receptor- $\gamma($ PPAR $\gamma)$ (Nagy et al., 1998; Tontonoz et al., 1998), nuclear erythroid-related factor 2 (Nrf2) (Ishii et al., 2004; Olagnier et al., 2011), as well as by signal transducer and activator of transcription 1 (STAT1) (Kotla et al., 2017) pathways. In addition, CD36 expression can also be regulated by retinol-binding protein 4 (Liu et al., $2017 \mathrm{~b})$, protein kinase $\mathrm{C} \theta(\mathrm{PKC} \theta) /$ activating transcription factor 2 (Raghavan et al., 2018), epidermal growth factor receptor (Zeboudj et al., 2018), trimethylamine
$N$-oxide (Geng et al., 2018), NACHT, LRR, and PYD domains-containing protein 3 inflammasome (Chen et al., 2018b), transient receptor potential vanilloid 4 (TRPV4) (Goswami et al., 2017), cellular communication network factor 3 (Shi et al., 2017), melanocortin 1 receptor (Rinne et al., 2017), cluster of differentiation 146 (CD146) (Luo et al., 2017), triggering receptor expressed on myeloid cells (Joffre et al., 2016), cyclophilin A (Ramachandran et al., 2016), and many others. Furthermore, CD36-mediated foam cell formation and atherosclerosis were negatively regulated by $\mathrm{C} 1 \mathrm{q} /$ tumor necrosis factor-related protein 13 via autophagy/lysosomedependent degradation of CD36 (Wang et al., 2019a).

Recently, noncoding RNAs (ncRNA), such as miRNAs and lncRNAs, were proposed as a new layer of gene regulation in cardiovascular biology. A few miRNAs have been shown to regulate the CD36 expression, thus influencing foam cell formation and lipid accumulation in macrophages (Dai et al., 2016), including miRNA-155 
(Huang et al., 2010). The CD36 expression is also regulated by lncRNAs. For example, lncRNA RP5833A20.1 interacted with miRNA-382-5p to suppress nuclear factor I A (NFIA), thus increasing the expression of nuclear transcription factor- $\kappa \mathrm{B}(\mathrm{NF}-\kappa \mathrm{B})$, SR-A, and CD36, leading to increased cholesterol accumulation and higher inflammatory response ( $\mathrm{Hu}$ et al., 2015). lncRNAs nuclear paraspeckle assembly transcript 1 (Neat1), which has two isoforms, Neat1_1 (shorter) and Neat1_2 (longer), regulate the formation of paraspeckles (subnuclear structures). Two recent studies have shown that Neat1 is an oxLDL inducible lncRNA that regulates the macrophage inflammation, oxidative stress, apoptosis, and foam cell formation (Chen et al., 2018a; Huang-Fu et al., 2018). Similarly, metastasisassociated lung adenocarcinoma transcript 1 (MALAT1), a macrophage-enriched and oxLDL inducible lncRNA, also involves in regulation of oxLDL-induced CD36 expression and oxLDL uptake through activation of $\beta$-catenin (a transcriptional factor for CD36) (Huangfu et al., 2018). Despite reduced uptake of oxLDL by MALAT1 deficiency, heterozygous deletion of MALAT1 aggravated atherosclerosis in ApoE-deficient $\left(\mathrm{ApoE}^{-/-}\right)$ mice by inducing massive immune system dysregulation (Gast et al., 2019). Gain- and loss-of-function assays of homeobox protein transcription antisense RNA (HOTAIR) in oxLDL-stimulated macrophages suggest that HOTAIR controlled CD36 expression, oxLDL uptake, inflammation [interleukin-6 (IL-6), IL-1 $\beta$, cyclooxygenase 2 (COX2), and tumor necrosis factor- $\alpha$ (TNF- $\alpha)$ ], and macrophagederived foam cell formation via miRNA-330-5p (Liu et al., 2019). Therefore, ncRNAs present potential targets to fine-tune macrophage function within atherosclerotic lesions. For a detailed review of ncRNAs in regulating SRs, we refer to a recent review (Dai et al., 2016).

Studies performed using CD36 $6^{-1-}$ mice to dissect the role of CD36 in atherosclerosis have yield controversial results (Collot-Teixeira et al., 2007). For example, targeted disruption of CD36 reduced the oxLDL uptake, foam cell formation, and atherosclerotic plaques in $\mathrm{ApoE}^{-1-}$ mice (Febbraio et al., 2000). This was supported by an independent study using shRNA-mediated gene silencing of CD36, which showed that silencing of CD36 in bone marrow-derived cells decreased atherosclerotic plaques (Mäkinen et al., 2010). Another study showed that CD36 ${ }^{-/-}$-deficient male $\mathrm{ApoE}^{-/-}$mice, but not female mice, showed reduced atherosclerotic plaque area after feeding normal diet and Western-type diet; however, this was unexpectedly related to increased aortic sinus lesion areas (Moore et al., 2005). In contrast, a second study observed that CD36 deficiency reduced atherosclerosis in both sexes of $\mathrm{ApoE}^{-1-}$ mice (Kuchibhotla et al., 2008). A third study observed increased atherosclerosis only in male CD36 ${ }^{-1-}$ and $\mathrm{ApoE}^{-/-}$mice fed a Western-type diet, albeit with reduced foam cell formation in vitro; however, simultaneous deletion of both CD36 and SR-A significantly reduced atherosclerosis as well as plaque necrosis in $\mathrm{ApoE}^{-/-}$ mice without affecting foam cell formation (ManningTobin et al., 2009). These discrepancies in phenotypic observations suggest that CD36 in other cell types (such as endothelial cells, VSMCs, monocytes, and dendritic cells) may have lipid uptake-independent functions, and the possible reciprocal interaction network among SRs may be complicatedly regulating lipid metabolism and atherosclerosis (Maguire et al., 2019).

2. Macrophage Scavenger Receptor 1. SR-A (also known as CD204, or macrophage scavenger receptor 1) is a 77-kDa glycoprotein belonging to class $\mathrm{A}$ of the $\mathrm{SR}$ family. SR-A is the first identified and cloned SR in macrophages (Kodama et al., 1990). Alternative splicing of SR-A leads to the generation of several variants, such as SR-A1/2/3 (human SR-A) and SR-A1/2 (mouse SR-A). SR-A gene is located on chromosome 8 (8p22). SR-A protein has a cysteine-rich C-terminal domain, an extracellular domain, and a collagen-like domain (McLaren et al., 2011a). SR-A1/2s are the common functional receptors that mediate the endocytosis of modified LDLs, such as acetylated LDL (acLDL) (Terpstra et al., 1997). The expression of SR-A was upregulated by multiple proatherogenic stimuli [such as acLDL (Terpstra et al., 1997), oxLDL (Terpstra et al., 1997), TNF- $\alpha$ (Hashizume and Mihara, 2012), IL-6 (Hashizume and Mihara, 2012), high glucose (Fukuhara-Takaki et al., 2005), etc.] and downregulated by many plant-derived phytochemicals (Yu et al., 2013). SR-A1 gene transcription is primarily regulated by PU.1 (Horvai et al., 1995), NF- $\kappa \mathrm{B}$ (Hashizume and Mihara, 2012), activating protein-1 (AP-1) (Mietus-Snyder et al., 1998), and CCAAT/ enhancer binding protein (C/EBP) (Mietus-Snyder et al., 1998). Several miRNAs were shown to regulate the SR-A expression, such as miRNA-29a (Dai et al., 2016). SR-A1 expression can be upregulated through Orai1 store-operated calcium channel (Liang et al., 2016), chloride channel $\mathrm{ClC}_{3}$ (Tao et al., 2015b), and endophilin-A2 (Huang et al., 2016a).

Earlier studies in the 1990s showed that deletion of SR-A can reduce atherosclerosis development in both LDL receptor-deficient $\left(\mathrm{LDLR}^{-/-}\right.$) mice (Sakaguchi et al., 1998) and ApoE ${ }^{-/-}$mice (Suzuki et al., 1997). The pathologic role of SR-A is supported by its silencing in $\mathrm{LDLR}^{-/-}$ApoB100 mice. However, simultaneous depletion of SR-A and CD36 did not reduce atherosclerosis, due to reciprocal regulation of both receptors (Mäkinen et al., 2010). Follow up studies suggested the more complex role of SR-A in atherosclerosis. For example, deficiency of either SR-A or CD36 alone had no impact on atherosclerotic development in $\mathrm{ApoE}^{-/-}$ mice, although it attenuated foam cell formation (Moore et al., 2005), but SR-A and CD36 double knockout reduced plaque complexity without significant impact on macrophage-derived foam cell formation. Also, SR-A transgenic in either $\mathrm{LDLR}^{-/-}$mice or ApoE3 Leiden mice decreased the atherosclerosis development 
despite increased foam cell formation in SR-A overexpressed macrophages (De Winther et al., 2000). This discrepancy may be attributed to several factors, including different diet, time of feeding, and different atherosusceptible mouse strain. To avoid the systemic effects caused by global knockout, macrophage-specific overexpression of SR-A was done and unexpectedly led to attenuated atherosclerosis development in $\mathrm{LDLR}^{-/-}$ mice (Whitman et al., 2002). Similarly, overexpressing SR-A in $\mathrm{LDLR}^{-1-}$ mice (via bone marrow transplantation) does not affect atherosclerotic lesion development despite increased SR activity in vitro (Herijgers et al., 2000). In addition to regulating lipid uptake, SR-A also has other biologic functions, such as regulation of vascular inflammation, host defense, innate immunity, cell fate determination, and ischemic injury, highlighting that SR-A could be a double-edged sword implicated in cardiovascular health as well as in multiple cardiovascular diseases (CVD) (Kelley et al., 2014; Ben et al., 2015).

3. Lectin-like Oxidized Low-density Lipoprotein Receptor-1. LOX-1 (lectin-like oxLDL receptor-1) belongs to the Class $\mathrm{E}$ of scavenger receptors located at chromosome 12 (12p13.2). LOX-1 is encoded by the gene-oxLDL receptor 1 (OLR1). LOX-1 was identified in 1997 as a major SR in endothelial cells that binds, internalizes, and degrades oxLDL (Sawamura et al., 1997). Genetic variants of LOX-1 were associated with increased risk of CVD (Chen et al., 2003; Wang et al., 2010c; Morini et al., 2016). The human LOX-1 gene has six exons and five introns, encoding LOX-1 protein, which has 273 amino acid residues. LOX-1 is composed of a C-type lectin-like domain, an extracellular domain, a transmembrane domain, and an $\mathrm{N}$-terminal cytoplasmic domain (Dunn et al., 2008; Ogura et al., 2009). The C-type lectin domain is critical for oxLDL binding. In terms of expression, LOX-1 is ubiquitously expressed in vascular cells, such as endothelial cells (Sawamura et al., 1997), macrophages (Draude et al., 1999), VSMCs (Zhang et al., 2017), cardiomyocytes (Schlüter et al., 2017), and fibroblasts (Liu et al., 2016a). The ubiquitous expression pattern of LOX-1 in cardiovascular cells implicates its important role in regulating cardiovascular pathophysiology. In patients with advanced atherosclerotic plaques, LOX-1 protein expression was markedly increased in lesional macrophages and VSMCs (Kataoka et al., 1999). In addition, LOX-1 is cleaved to form soluble LOX-1, which serves as a diagnostic and prognostic biomarker for CAD (Tian et al., 2019a).

To date, oxLDL is the most common and wellcharacterized ligand for LOX-1 (Yoshimoto et al., 2011). Other ligands of LOX-1 include carbamylated LDL (Speer et al., 2014; Holy et al., 2016), glycoxidized LDL (Shiu et al., 2009), electronegative LDL fraction L5 (Wang et al., 2018g), and advanced glycation end-products (Jono et al., 2002). Expression of LOX-1 under basal unstimulated conditions is very low, but LOX-1 expression can be significantly upregulated by several atheroprone stimuli, such as disturbed blood flow (Lee et al., 2018), lipopolysaccharide (LPS) (Zhao et al., 2014), TNF- $\alpha$ (Kume et al., 2000), and high glucose ( $\mathrm{Li}$ et al., 2003). Dysfunctional HDL from patients with CAD can also cause endothelial dysfunction by reducing endothelial nitric oxide (NO) synthase (eNOS)-dependent NO production, anti-inflammatory responses, and endothelial repair capacity via LOX-1 activation (Besler et al., 2011; Xu et al., 2013b). Similarly, dysfunction of HDL could probably lead to LOX-1-mediated uptake of modified LDL and result in foam cell formation.

LOX-1 gene transcription can be regulated by several transcriptional factors, including NF- $\kappa \mathrm{B}, \mathrm{AP}-1$, and POU-domain transcription factor (Oct-1) in a contextdependent manner (Hermonat et al., 2011). LOX-1 gene expression can be epigenetically mediated by several miRNAs. For example, silencing of miRNA-155 promoted oxLDL-elicited lipid uptake by increasing the expression of LOX-1 (Huang et al., 2010). A recent miRNA transcriptional profiling assay showed that LOX-1 is the target of miRNA-30c-1-3p and miRNA28a-5p in oxLDL-stimulated RAW264.7 macrophages (Li et al., 2018b). Sirtuin 1 (Sirt1), a class III histone deacetylase, can downregulate LOX-1 expression and LOX-1-mediated foam cell formation, as well as attenuate development of atherosclerosis by deacetylating in macrophages (Stein and Matter, 2011). It remains to be investigated whether there are lncRNAs that can regulate LOX-1 expression and LOX-1-mediated foam cell formation.

LOX-1 transgene accelerates endothelial dysfunction, aortic inflammation, intramyocardial vasculopathy, and atherosclerotic development in $\mathrm{ApoE}^{-/-}$mice (Inoue et al., 2005). Experiments using endothelial cell-specific overexpression of LOX-1 obtained similar phenotype in either $\mathrm{ApoE}^{-/-}$or $\mathrm{LDLR}^{-/-}$mouse background by impairing endothelial function (White et al., 2011; Hofmann et al., 2017). In contrast, LOX-1 ${ }^{-1-}$ and $\mathrm{LDLR}^{-/-}$mice show reduced collagen deposition, oxidative stress, and developed fewer atherosclerotic plaques (Mehta et al., 2007; Hu et al., 2008). These series of gain- and loss-of-function studies provide the "proof-ofconcept" that LOX-1 is a proatherogenic SR and can serve as a promising therapeutic target for atherosclerosis. The molecular mechanisms of LOX-1 in promoting atherosclerosis include induction of endothelial dysfunction (injury, apoptosis, impaired NO production, and endothelial-mesenchymal transition), the proliferation/ migration of VSMCs, macrophage-derived foam cell formation, and platelet activation (Tian et al., 2019a).

For a detailed understanding of the functions and pharmacological modifiers of LOX-1 in atherosclerosis, we refer the readers to a recently published review 
(Tian et al., 2019a). More studies are needed to understand the specific role of LOX-1 in macrophage foam cell formation and atherosclerosis (by macrophagespecific knockout mice and/or transgenic mice) (Maguire et al., 2019). Also, LOX-1 was expressed in VSMCs in mice and human atherosclerotic plaques, the potential role of LOX-1 in lipid uptake and foam cell formation in VSMCs remains to be investigated.

4. Others. Other SRs that mediate lipid uptake include MARCO (Elomaa et al., 1998), SR expressed in endothelial cells (Adachi et al., 1997), and CXCL16 (Shimaoka et al., 2000). For example, CXCL16 was a SR highly expressed in macrophages (Shimaoka et al., 2000). Knockdown experiments have demonstrated that CXCL16 was important for foam cell formation in human macrophages (Zhang et al., 2008). It is plausible that these less common SRs also contribute to the uptake of modified LDL to certain extent or under specific disease conditions (McLaren et al., 2011a). Further studies are warranted to assess the contribution of these SRs to foam cell formation and atherosclerosis in genetically manipulated mice.

\section{B. Cholesterol Esterification and Hydrolysis}

After uptake and internalization by macrophage SRs, modified LDL is transported to the late endosome/ lysosomes where the cholesteryl esters (CEs) are further hydrolyzed to free cholesterol (FC) and free fatty acids by lysosomal acid lipase [LAL, also known as lipase A (LIPA)] (Du et al., 2004; Ouimet et al., 2011; Dubland and Francis, 2015; Schlager et al., 2017). To prevent the potential cell toxicity caused by an excessive FC accumulation, FC can be re-esterified by acetyl-CoA acetyltransferases (ACAT) at the endoplasmic reticulum (ER) to form CE, which is stored in cytoplasm in the form of lipid droplets (LD). Optimal cholesterol esterification is regarded as a protective self-defense mechanism to avoid the excessive accumulation of cytotoxic FC. The excessive accumulation of $\mathrm{CE}$ in macrophages and VSMCs leads to the formation of foam cells, which represents a major hallmark for the development of atherosclerosis. The accumulated $\mathrm{CE}$ within the cells can be hydrolyzed by two neutral CE hydrolases (NCEH), termed NCEH1 (also known as KIAA1362) (Okazaki et al., 2008; Sekiya et al., 2009) and hormone-sensitive lipase (HSL, also known as lipase E) (Escary et al., 1998, 1999; Okazaki et al., 2002; Yeaman, 2004). The result is the release of $\mathrm{FC}$ for transporters-mediated efflux. Therefore, preventing cholesterol esterification and stimulating CE hydrolysis are crucial for maintaining cholesterol homeostasis, and therefore, represent novel therapeutic modalities of atherosclerosis.

1. Acetyl-CoA Acetyltransferases 1 and Acetyl-CoA Acetyltransferases 2. ACAT has two isoforms: ACAT1 (which is expressed in macrophages, VSMCs, and other cell types) and ACAT2 (mainly expressed in the intestinal enterocytes, macrophages, and hepatocytes).
ACAT2 has $44 \%$ sequence similarity with ACAT1 (Cases et al., 1998; Lee et al., 2000b; Sakashita et al., 2003; Allahverdian et al., 2012). ACAT1 was first identified in 1993 by Chang et al. (1993) and is the well-studied ACAT isoform in mediating CE synthesis. ACAT1 is located in chromosome 11 (11q22.3). Human ACAT1 gene spans $27 \mathrm{~kb}$ and has 12 exons and 11 introns. ACAT1 gene encodes a $45.1-\mathrm{kDa}$ product, which is composed of 427 amino acids. ACAT1 was predominantly expressed in plaque macrophages in human patients, and its expression was significantly increased during monocyte differentiation to macrophages (Miyazaki et al., 1998). Moreover, several proatherogenic stimuli, including interferon- $\gamma$ (Panousis and Zuckerman, 2000b), oxLDL (Wang et al., 2016b), TNF- $\alpha$ (Lei et al., 2009), insulin (O'Rourke et al., 2002), leptin (O'Rourke et al., 2002), Chlamydia pneumoniae infection (Liu et al., 2010a), and asymmetric dimethylarginine (Zhu et al., 2010), upregulated the expression/activity of ACAT1 in macrophages. Furthermore, cholesterol loading of aortic VSMCs also leads to increase in ACAT1 activity, while ACAT2 was not expressed by arterial VSMCs (Rong et al., 2005). Also, oxLDL increases the ACAT1-dependent foam cell formation in VSMCs via toll-like receptor 4 (TLR4)/MyD88/NF- $\kappa$ B-mediated proinflammatory pathways (Yin et al., 2014). In contrast, the ACAT1 expression in macrophages can be decreased by ghrelin [via growth hormone secretagogue receptor (Wan et al., 2009)], incretins [via cyclic AMP (cAMP) activation (Nagashima et al., 2011)], and the vasoprotective gasotransmitter hydrogen sulfide $\left(\mathrm{H}_{2} \mathrm{~S}\right)$ [via $\mathrm{K}_{\mathrm{ATP}} / \mathrm{ERK} 1 / 2$ pathway (Zhao et al., 2011)]. The expression of ACAT1 can be regulated by a few miRNAs, including miRNA-9 (Xu et al., 2013a), miRNA-27a/b (Zhang et al., 2014), and miRNA-467b (Wang et al., 2017a), all of which reduced the macrophage-derived foam cell formation. Clinically relevant, two genetic variants of ACAT1 gene (such as rs1154556 and rs10913733) were associated with an increased risk of CAD in Chinese population (Wang et al., 2017i).

A large part of the scientific knowledge about ACAT1 and ACAT2 has been gained from genetically engineered mice by breeding with atherosusceptible mouse strains. Total deficiency or liver-specific deletion (by administering antisense oligonucleotide targeting ACAT2) of ACAT2 has consistently reduced atherosclerosis in mice (Willner et al., 2003; Bell et al., 2006, 2007), whereas the outcome of deletion/pharmacological inhibition of ACAT1 in experimental atherosclerosis is controversial (Rudel et al., 2005; Farese, 2006). On the one hand, global deletion of ACAT1 in either $\mathrm{ApoE}^{-/-}$or $\mathrm{LDLR}^{-/-}$mice show reduced CE accumulation and atherosclerosis development, but caused cutaneous xanthomatosis and dry eye in mice, probably due to an excessive FC accumulation (Yagyu et al., 2000). Similarly, myeloid-specific ACAT1 deficiency (by breeding with LyzM-Cre mice) reduces the content of $\mathrm{CE}$ and 
leukocyte adhesion to activated endothelium, lesional macrophage content, and atherosclerosis plaque area in $\mathrm{ApoE}^{-/-}$mice, without causing common side effects caused by the ACAT1 total deficiency (Huang et al., 2016b). Myeloid-specific ACAT1 ablation also reduces macrophage inflammation and prevents diet-induced obesity (Huang et al., 2018). On the other hand, myeloid cell-specific deletion of ACAT1 (by bone marrow transplantation) unexpectedly show accelerated atherosclerosis in both $\mathrm{ApoE}^{-/-}$(Su et al., 2005) and $\mathrm{LDLR}^{-/-}$ mice (Fazio et al., 2001). Pharmacological inhibition of ACAT by inhibitors increases atherosclerotic lesion area in $\mathrm{ApoE}^{-/-}$mouse and rabbit models of atherogenesis (Perrey et al., 2001). These unexpected findings are probably due to a FC-induced cytotoxicity and an impairment of cholesterol efflux (Fazio and Linton, 2006).

Diverse pharmacological inhibitors of ACAT have been explored as effective therapeutic strategies to assess the therapeutic potential of ACAT inhibition in atherosclerosis. Most of these inhibitors have shown promising results in ameliorating atherosclerosis development in animal models. For example, ACAT inhibitors, such as avasimibe (Nicolosi et al., 1998; Delsing et al., 2001; Kharbanda et al., 2005), CS-505 (Terasaka et al., 2007), T-2591 (Yasuhara et al., 1997), HL-004 (Ishii et al., 1998), K604 (Yoshinaka et al., 2010), F1394 (Rong et al., 2013), and tomatidine (Fujiwara et al., 2012), have been reported to inhibit foam cell formation and atherosclerosis in hyperlipidemic mice. Some ACAT inhibitors, like avasimibe, have been shown to reduce the systemic inflammation and improve the endothelial functions in patients with hypercholesterolemia (Kharbanda et al., 2005). Most of these inhibitors partially inhibited the ACAT activity and both isoforms of ACAT. Recently, Shibuya et al. (2018) identified a highly potent ACAT1 inhibitor $\left(\mathrm{IC}_{50}=4.0 \mathrm{nM}\right)$, which reduced lipid-accumulation in the aortic arch of hamsters fed with an atherogenic diet, raising the therapeutic potential of this compound to attenuate atherosclerosis in other experimental animal models and human patients with atherosclerosis. Other recent studies have shown that other treatment options, such as losartan (Rafatian et al., 2013) or inhibition of the NLRP3 inflammasome (by compound MCC950) (Chen et al., 2018b), inhibit the ACAT activity, CE accumulation, and foam cell formation. However, three randomized, double-blind, placebo-controlled trials failed to observe significant differences in carotid as well as coronary atherosclerosis between treatment with ACAT inhibitors (by pactimibe and avasimibe) and placebo (Tardif et al., 2004; Nissen et al., 2006; Meuwese et al., 2009). The evidence indicates that selective ACAT inhibition might be desired and should be used in combination with therapies that increase cholesterol acceptors (such as HDL or ApoA-1 targeted therapies), driving out excessive FC of the plaques (Fazio and Linton, 2006).
2. Neutral Cholesterol Ester Hydrolase 1. NCEH1 gene is located at chromosome 3 (3q26.31) in the ER. Compared with HSL, NCEH1 contributes more to CE hydrolysis in macrophages (Sakai et al., 2014). NCEH1 gene yields four isoforms that result from differences in mRNA cleaving. Structurally, NCEH1 is a membrane protein consisting of an $\mathrm{N}$-terminal transmembrane domain, a catalytic domain, and C-terminal lipid-binding domains (Okazaki et al., 2008). The N-terminal domain has an ER-anchoring sequence. NCEH1 gene was first cloned and biologically characterized in macrophages (Ghosh, 2000; Okazaki et al., 2008). It was recently shown that estrogen significantly increases the activity of NCEH1 in mouse macrophages, suggesting the possibility of resulting sex difference of atherosclerosis development (Chiba et al., 2011).

To dissect the role of NCEH1 in foam cell formation and atherosclerosis, Igarashi et al. (2010) showed that NCEH overexpression enhanced the CE hydrolysis and promoted cholesterol efflux from macrophages. In contrast, the NCEH1 depletion promoted foam cell formation, indicating the critical role of NCEH1 in maintaining cholesterol homeostasis (Okazaki et al., 2008). In vivo, NCEH1 deletion accelerated foam cell formation and increased atherosclerotic plaque area in $\mathrm{ApoE}^{-/-}$mice without impacting lipid profile. NCEH1 and HSL1 have comparable NCEH activity in macrophages despite one contradictory report (Buchebner et al., 2010), which showed that HSL deficiency, but not NCEH1 deficiency, abolished the NCEH activity. Mice with deficiency of both NCEH1 and HSL1 showed exaggerated atherosclerotic plaque increase in an additive manner (Sekiya et al., 2009). In contrast, mice overproducing both NCEH and ApoA4 (as cholesterol acceptor) showed decreased atherosclerosis development (Choy et al., 2003). Also, macrophage-specific overexpression of NCEH1 reduced atherosclerotic lesion area and plaque necrosis in $\mathrm{LDLR}^{-/-}$mice due to enhanced FC efflux and reverse cholesterol transport (RCT) (Zhao et al., 2007). Excessive accumulation of FC or oxysterols induces macrophage apoptosis and atherosclerosis. NCEH1-deficient, but not HSL-deficient, macrophages were more sensitive to 25 -hydroxycholesterol-induced apoptosis (Sekiya et al., 2014). Therefore, NCEH1 not only reduced the CE content in foam cells, but also prevented the oxysterols-induced apoptosis in atherosclerosis (Sekiya et al., 2014). Overall, these results highlight NCEH1 as a promising target for treating atherosclerosis.

3. Lysosomal Acid Lipase (Lipase A) and Hormonesensitive Lipase 1 (Lipase E). In addition to NCEH1, LAL is also a principal lipase that hydrolyzes lysosomal CE derived from LDL and modified LDL (Dubland and Francis, 2015). Genome-wide association studies identified several genetic variants of LAL gene, which was associated with increased risk for CAD (Morris et al., 2017). In mice, intravenous administration of recombinant 
LAL reduced coronary and aortic atheromatous lesions in $\mathrm{LDLR}^{-1-}$ mice fed an atherogenic diet (Du et al., 2004). A recent study has shown that LAL promotes $\mathrm{RCT}$ in cultured macrophages and in mice by increasing the expression of $\mathrm{ABCA} 1$ and ABCG1 (Bowden et al., 2018).

Unlike LAL, HSL is a neutral lipase expressed in macrophages. Macrophage-specific HSL-transgenic mice show enlarged aortic lesions and increased lipid accumulation in coronary arteries (Escary et al., 1999). However, the overexpression of cholesterol acceptors (ApoA4) reduces atherosclerosis in HSL-transgenic mice (Choy et al., 2003). Studies using macrophages and $\mathrm{LDLR}^{-/-}$mice have indicated that HSL has comparable neutral CE hydrolase activity to those of NCEH1. The deletion of bone marrow-derived HSL increased a diet-induced atherosclerosis (Sekiya et al., 2009).

To date, pharmacological activators of LAL and HSL1 are not available. The specific role of LAL and HSL in foam cell formation of macrophages and VSMCs, as well as eventual effect on atherosclerosis remains to be investigated in future studies.

\section{Macrophage Cholesterol Efflux}

To maintain intracellular cholesterol homeostasis, excessive cholesterol is removed from cells through multiple pathways, with the cholesterol export process being termed "macrophage cholesterol efflux." The $\mathrm{CE}$ stored in the $\mathrm{LD}$ can be hydrolyzed to $\mathrm{FC}$ by $\mathrm{CE}$ hydrolase (Hopkins, 2013). Then, FC could be outflowed from macrophages by aqueous diffusion and by ATP-binding cassette (ABC) transporters ABCA1, ABCG1, and SR-BI (Rosenson et al., 2012; Chistiakov et al., 2016a). Caveolins (Murata et al., 1995) and sterol 27-hydroxylase (CYP27A1) (Escher et al., 2003) are also known as contributors to cholesterol efflux. ABCA1 promotes FC efflux to lipid-poor apolipoprotein A-1 (ApoA-1) (often referred to as pre- $\beta$ HDL) or apolipoprotein $\mathrm{E}(\mathrm{ApoE})$. ABCG1 can promote $\mathrm{FC}$ efflux to mature HDL (HDL2 and HDL3) particles (Hopkins, 2013). ABCA1 and ABCG1 expression are positively regulated by the nuclear receptor liver $\mathrm{X}$ receptor (LXR), which forms a heterodimer by binding with the retinoid $X$ receptor (RXR), and acts as a transcription factor. The expression of ABCA1, ABCG1, SR-BI, and LXR can also be induced by activation of PPAR $\gamma$ (Lusis, 2000; Hopkins, 2013). If cholesterol cannot be exported from cells to a sufficient extent, macrophages are transformed into foam cells (Hansson, 2005; Hansson et al., 2006).

1. Aqueous Diffusion Efflux Pathway. Aqueous diffusion efflux pathway recently was reviewed elsewhere (Phillips, 2014). This pathway involves a simple diffusion process, representing in this way the nonproteinmediated cholesterol efflux pathway. It was shown that it is one major contributor to cholesterol efflux particularly in normal mouse peritoneal macrophages
(MPMs) ( 80\%) (Adorni et al., 2007). However, in cholesterol-loaded MPMs, aqueous diffusion was not changed and its contribution to cholesterol efflux became smaller (Adorni et al., 2007). In addition, the major variations in cellular cholesterol efflux rates are not due to aqueous diffusion efflux pathway, but to membrane transporters-mediated cholesterol efflux pathways (Phillips, 2014).

\section{Transporter-Dependent Cholesterol Efflux}

Pathway. A significant pathway for cholesterol efflux from macrophages involves the interaction between transporters (ABCA1 and ABCG1), SR-BI, and acceptors (ApoA-1, HDL, and ApoE) (Brunham et al., 2006; Rosenson et al., 2011). It is proposed that ABCA1 and ABCG1 can act in a sequential way, in which ABCA1 generates nascent HDL, which then further promotes cholesterol efflux via ABCG1 (Gelissen et al., 2006). Genetic knockdown studies indicate that ABCG1 and ABCA1 account for about $20 \%$ and $50 \%$ of the net cholesterol efflux from cholesterol-enriched MPMs, respectively (Adorni et al., 2007). Furthermore, both ABCA1 and ABCG1 together account for about $60 \%-70 \%$ of the net cholesterol efflux to serum or HDL from LXR-activated macrophages loaded with cholesterol (Yvan-Charvet et al., 2007a). This part of the review will focus on current understanding of the function and regulation of these transporters (ABCA1 and ABCG1), SR-BI, and the acceptors (ApoA-1, HDL, and $\mathrm{ApoE}$ ), which are related to cholesterol efflux from macrophages.

a. ATP-binding Cassette Transporter A1. ABCA1 is a member of the $A B C$ transporter superfamily that utilizes ATP as a source of energy to transport various substrates across cellular membranes (Dean et al., 2001). ABCA1, originally named as ABC1, was identified by a PCR-based approach and cloned in 1994 (Luciani et al., 1994). The ABCA 1 gene consists of 50 exons spanning $149 \mathrm{~kb}$ and encodes a 2261 amino acid integral membrane protein, consisting of two transmembrane domains (TMDs) with six helices each and two nucleotide-binding domains (NBDs) (Santamarina-Fojo et al., 2000; Wang and Smith, 2014). The NBDs exhibit a nucleotide-free state, while the two TMDs link each other via a narrow interface in the membrane. Additionally, two extracellular domains of ABCA1 form an elongated hydrophobic tunnel. There are two large extracellular loops that are linked by a disulfide linkage (Fitzgerald et al., 2002; Qian et al., 2017).

It was not until 1999 that mutations of ABCA1 gene were identified as underlying the etiology of Tangier disease (Rust et al., 1999), which is characterized in the homozygous state by an HDL deficiency, frequently premature $\mathrm{CAD}$, extremely enlarged yellow tonsils, and clouding of the cornea (Bale et al., 1971; SerfatyLacrosniere et al., 1994; Rust et al., 1999). Further studies indicate that ABCA1 plays a major role in HDL biosynthesis by mediating FC and phospholipid efflux to lipid-poor ApoA-1, thereby producing nascent HDL 
particles in the liver (Yokoyama, 2006; Phillips, 2014). Fibroblasts from subjects with Tangier disease are deficient in cholesterol efflux to ApoA-1 (Remaley et al., 1997), indicating that ABCA1 is an important transporter mediating the cholesterol efflux. Details about the interaction of the ABCA1 protein with ApoA-1 in mediation of the cholesterol efflux were reviewed recently (Wang and Smith, 2014; Phillips, 2018). To date, there is no clear consensus regarding the process by which the ABCA1 protein regulates the transport of cholesterol and lipoprotein from the plasma membrane to the acceptor ApoA-1.

Consistent with the proposed function of ABCA1 in nascent HDL formation, ABCA1-deficient mice have almost undetectable levels of HDL, with a significant decrease in total plasma cholesterol and phospholipids (McNeish et al., 2000; Orsó et al., 2000). Overexpression of ABCA1 in mice led to increased HDL-cholesterol (HDL-C) and ApoA-1 levels, facilitating hepatic RCT and biliary cholesterol excretion (Singaraja et al., 2001; Vaisman et al., 2001). However, the studies in vivo from some groups have led to opposite results about the role of ABCA1 expression in atherosclerosis development. Complete ABCA1 deficiency in wild-type, $\mathrm{LDLR}^{-/-}$, and $\mathrm{ApoE} \mathrm{E}^{-/-}$mice did not alter progression of atherosclerosis (McNeish et al., 2000), but repopulation of $\mathrm{ABCA} 1^{-/-}$mice with wild-type macrophages (McNeish et al., 2000) or macrophages from ABCA1-overexpressing mice (McNeish et al., 2000) led to a significant decrease of atherosclerotic lesion in mice. In addition to lipid regulation, ABCA1 protein is also involved in the regulation of apoptosis and inflammation (Soumian et al., 2005). ABCA1 executes its anti-inflammatory effects by modifying cell membrane lipid rafts and directly activating signaling pathways, including Janus kinase 2/STAT3, protein kinase A (PKA), Rho family G protein CDC42, and PKC (Liu and Tang, 2012).

ABCA1 protein plays a critically important role in cholesterol homeostasis and may be viewed as a protector against atherosclerosis, thus there are many studies to decipher how its expression is regulated at both transcriptional and posttranscriptional levels (Schmitz and Langmann, 2005; Zarubica et al., 2007) (Fig. 3). ABCA1 expression is regulated in a tight pathway with a short protein half-life (1 to 2 hours) (Yokoyama et al., 2012) and rapid turnover in macrophages (Soumian et al., 2005). ABCA1 protein expression is highly regulated by a variety of molecules, including secondary messengers (e.g., cAMP), nuclear receptors [e.g., LXR, RXR, PPAR, pregnane $\mathrm{X}$ receptor (PXR)], and cytokines [e.g., TNF- $\alpha$, transforming growth factor- $\beta$ (TGF- $\beta$ ), interleukin- $1 \beta$ (IL-1 $\beta$ )] (Zarubica et al., 2007). cAMP upregulates the expression of ABCA1 protein by acting at both the transcriptional and translational levels (Haidar et al., 2002; Denis et al., 2003). Nuclear receptors are ligand-dependent transcriptional factors that mediate the expression of their target genes
(Beaven and Tontonoz, 2006). The activation of LXR and RXR by physiologically occurring oxysterols (e.g., 27-hydroxycholesterol), retinoids, or synthetic agonists stimulates the transcription of ABCA1 via the DR4 element in the ABCA1 promoter (Zarubica et al., 2007). PPAR upregulates ABCA1 expression and RCT indirectly via enhancement of the transcription of LXR $\alpha$ (Chinetti et al., 2001). PXRs, regulated by many compounds including both natural and synthetic steroids, downregulate the ABCA1 gene transcription (Sporstol et al., 2005). Cytokines were shown to exhibit pleiotropic and contradictory effects on ABCA1 expression by cross-talks between the cellular process of cholesterol and inflammatory reactions (Zarubica et al., 2007). TNF- $\alpha$, IL- $1 \beta$, and interferon- $\gamma$ downregulate the LXR-mediated increase of ABCA1 protein expression (Lusis, 2000; Panousis and Zuckerman, 2000a), whereas TGF- $\beta$ positively regulates the ABCA1 expression (Panousis et al., 2001). Posttranscriptional regulation also plays a critical role in the regulation of ABCA1 protein levels by adjusting either protein stability or its transporter activity (Llaverias et al., 2005). It was reported that the protein stability of ABCA1 is regulated by the proteasomal, lysosomal, and calpain systems (Ogura et al., 2011; Yokoyama et al., 2012; Aleidi et al., 2015), whereas its activity is under the fine control of diverse protein kinases, such as PKA and protein kinase CK2 (Haidar et al., 2004; Roosbeek et al., 2004). It was shown that a proline, glutamic acid, serine, and threonine sequence in ABCA1 is very important for its degradation by calpain protease, since deletion of this motif leads to increase ABCA1 protein levels by four- to fivefold (Wang et al., 2003).

The regulation of ABCA1 protein expression by miRNA was recently investigated. miRNAs, are a class of ncRNAs that are predominantly posttranscriptional regulators of gene expression (Ambros, 2004; Bartel, 2009). To date, miRNA-10b (Wang et al., 2012a), miRNA-23a-5p, miRNA-33 (Rayner et al., 2010), miRNA-106b (Kim et al., 2012), miRNA-128-2 (Adlakha et al., 2013), miRNA-148a (Goedeke et al., 2015), miRNA-183 (Sarver et al., 2010), and miRNA-758 (Ramirez et al., 2011) have been reported to regulate the ABCA1 protein expression. MiRNA-10b directly repressed $\mathrm{ABCA} 1$ expression and also suppressed cholesterol efflux from both murine and human macrophages (Wang et al., 2012a). Transfection of miRNA23a-5p inhibitor enhanced cholesterol efflux and decreased foam cell formation through upregulating the ABCA1 expression levels. miRNA-23a-5p reduced ABCA1 expression via repressing the 3 '-untranslated regions (UTR) activity of the ABCA1 transcripts. Longterm in vivo systemically delivered miRNA-23a-5p antagomir significantly increased ABCA1 expression, reduced atherosclerosis development, and promoted plaque stability in the aorta of $\mathrm{ApoE}^{-1-}$ mice (Yang et al., 2018a). miRNA-33, located within the gene encoding 
sterol regulatory element-binding factor-2, inhibited ABCA1 expression, thus resulting in the decreased cholesterol efflux (Rayner et al., 2010). miRNA-128-2 inhibited the ABCA1 expression directly via binding to its 3 '-UTR. The administration of miRNA-128-2 led to the decrease both protein and mRNA levels of ABCA1 (Adlakha et al., 2013). miRNA-148a decreased expression of ABCA1 and circulating HDL-C levels in vivo (Goedeke et al., 2015). miRNA-758 directly targeted the 3 '-UTR of ABCA1, repressed the expression of ABCA1, and reduced cellular cholesterol efflux to ApoA-1. Conversely, the application of anti-miRNA-758 increased ABCA1 expression (Ramirez et al., 2011). miRNA-106b significantly decreased the ABCA1 levels and the cholesterol efflux in neuronal cells (Kim et al., 2012). miRNA-183 targeted the 3 '-UTR of ABCA1 mRNA to degrade ABCA1 mRNA in the colon cancer cells and other tumor cells (Sarver et al., 2010). There has been no further study to investigate the effect of miRNA-106b and miRNA-183 on macrophages and liver cells so far.

Recent studies also showed that lncRNA, a large subgroup of RNAs that are $>200$ nucleotides. Although most lncRNAs do not have apparent protein coding potential, lncRNAs also play a very important role in modulating ABCA1 protein expression. lncRNAs can regulate the $A B C A 1$ expression at both transcriptional and posttranscriptional levels. It was reported that lncRNA macrophage-expressed LXR-induced sequence (MeXis) increased the ABCA1 protein expression at the transcriptional level by interacting with and guiding promoter to bind to the transcriptional coactivator DDX17 in macrophages (Sallam et al., 2018). Furthermore, the loss of MeXis in mouse bone marrow cells impaired the cellular reaction to cholesterol overload and accelerated the atherosclerotic development (Sallam et al., 2018). Increased long intervening noncoding RNA-DYNLRB2-2 expression promoted the ABCA1-mediated cholesterol efflux by upregulation of the ABCA1 expression through the glucagon-like peptide-1 (GLP-1) receptor signaling pathway (Hu et al., 2014) and decrease of TLR2 expression ( $\mathrm{Li}$ et al., 2018c) in macrophages. A newly identified lncRNA named lnc-HC, which interacts with hnRNPA2B1 to form an lnc-HC-hnRNPA2B1 complex, decreased the ABCA1 expression at the posttranscriptional level within hepatocytes (Lan et al., 2016). However, the effect of lnc$\mathrm{HC}$ on $\mathrm{ABCA} 1$ expression in macrophages remains to be examined. On the contrary, another lncRNA, RP5833A20.1, attenuated the ABCA1 levels, thus reducing

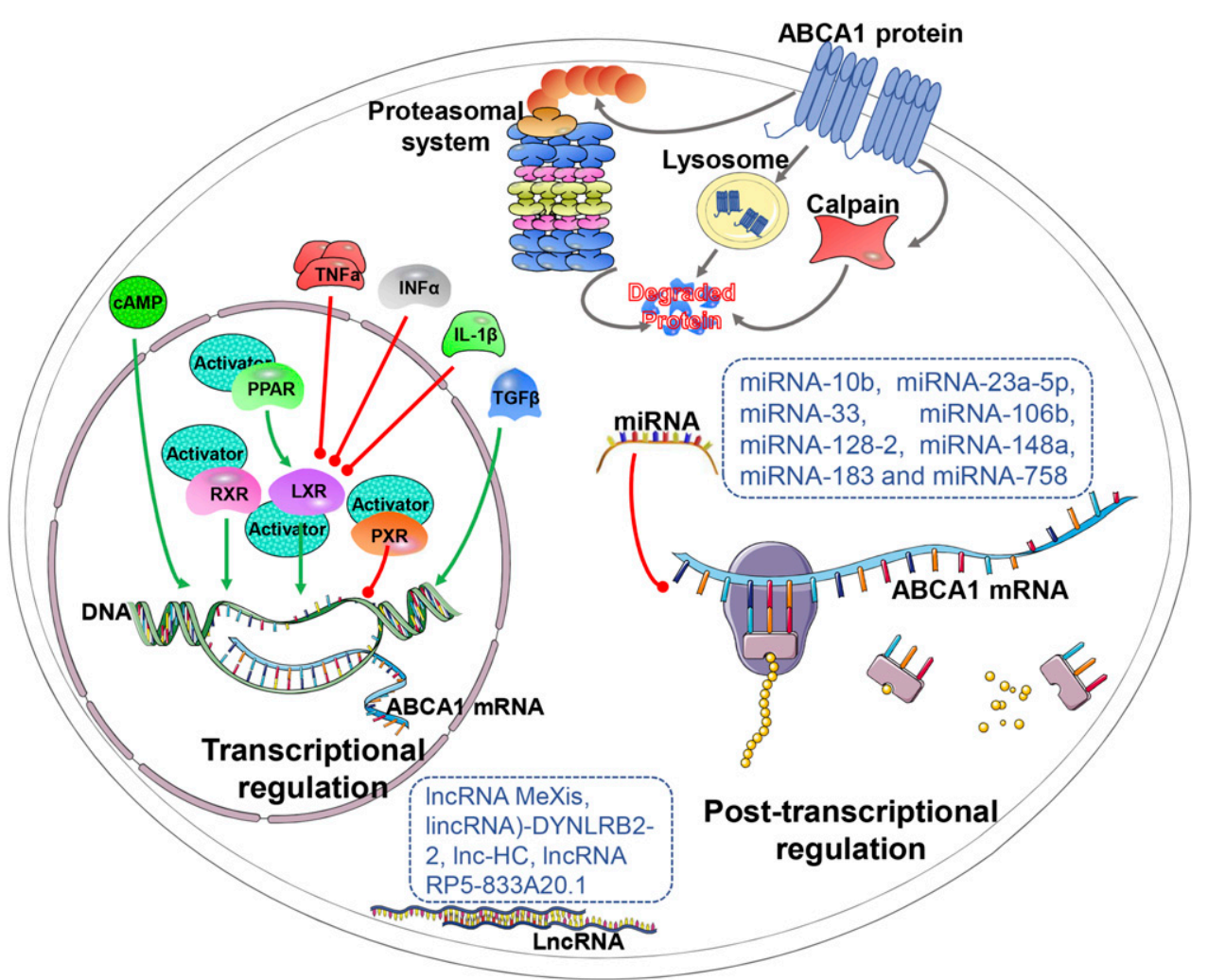

Fig. 3. Regulation of ABCA1 expression at both transcriptional and post-transcriptional levels. At the transcriptional level, ABCA1 protein expression is highly regulated by a variety of molecules, including secondary messengers [e.g., cyclic AMP (cAMP)], nuclear receptors (e.g., LXR, RXR, PPAR, and PXR), and cytokines [e.g., TNF- $\alpha$, transforming growth factor- $\beta$ (TGF- $\beta$ ), interleukin- $1 \beta$ (IL- $1 \beta)$ ]. At the post-transcriptional level, the protein stability of ABCA1 is governed by the proteasomal, lysosomal, and calpain systems. Also, miRNA-10b, miRNA-23a-5p, miRNA-33, miRNA-106b, miRNA-128-2, miRNA-148a, miRNA-183, and miRNA-758 have been reported to regulate ABCA1 protein expression. The long noncoding RNAs (lncRNAs), including lncRNA MeXis, long intervening lincRNA-DYNLRB2-2, lnc-HC, and lncRNA RP5-833A20.1, have also been involved in the regulation of ABCA1 expression (green arrows: upregulation; red lines: downregulation). 
the cholesterol efflux via the miRNA-382-mediated NFIA pathway (Hu et al., 2015).

b. ATP-binding Cassette Transporter G1. ABCG1 is another member of the ABC superfamily of transporters (Tarr et al., 2009; Tarling and Edwards, 2012). ABCG1 is a half transporter that contains a single $\mathrm{ABC}$ and a single TMD/6 transmembrane $\alpha$-helice on one polypeptide chain (Tarling, 2013). The half transporter ABCG1 is generally considered to function by forming homodimers (ABCG1:ABCG1) to transport substrates across the cell membrane (Tarling and Edwards, 2011). ABCG1 was first described and cloned in 1996 as the human homolog of the Drosophila white gene (Chen et al., 1996). After that, it took around 4 years until ABCG1 again received intensive attention because it is similar to ABCA1 in the expression pattern in monocytes (Schmitz et al., 2001). ABCG1 is involved in regulation of intracellular cholesterol homeostasis and cholesterol efflux from cells to HDL particles for RCT (Rosenson et al., 2012). ABCG1 also effluxes cholesterol to LDL, liposomes, and cyclodextrin (Wang et al., 2004; Kennedy et al., 2005) and it exports sphingomyelin, phosphatidylcholine, and oxysterols to HDL and albumin (Kobayashi et al., 2006; Xu et al., 2009). In addition to regulation of cholesterol efflux from cells, ABCG1 protein exhibits other physiologic functions as well (Sano et al., 2014). For example, ABCG1 is involved in cell proliferation, apoptosis, and immune response (Sano et al., 2014). It has been shown that ABCG1 suppresses the proliferation of $\mathrm{T}$ cells by LXR signaling (Bensinger et al., 2008). ABCG1 also induced the apoptosis of cultured cells (Seres et al., 2008), but inhibited the apoptosis of macrophages and prostate cancer cells by decreasing signaling of TLR4 and NADPH oxidase 2 (Yvan-Charvet et al., 2010a) and downregulating Akt signaling (Yvan-Charvet et al., 2010a), respectively.

Consistent with the function of ABCG1 in regulation of macrophage cholesterol efflux from cultured macrophages, ABCG1-deficient mice, first described in 2005, when fed a Western-type diet, displayed excessive lipid accumulation in macrophages within multiple organs, particularly in the lung (Kennedy et al., 2005). Unexpectedly, whole body loss of ABCG1 or overexpression of human ABCG1 had no influence on plasma lipid or lipoprotein levels (Kennedy et al., 2005; Burgess et al., 2008), which are in stark contrast to loss of ABCA1. Deeper insights about the coordinated participation of ABCA1 and ABCG1 in regulation of cholesterol efflux from macrophage have been obtained from animal studies (Rosenson et al., 2012). The combined deficiency of ABCA1 and ABCG1 resulted in markedly accelerated atherosclerotic lesion development in mice compared with the deficiency of either ABCA1 or ABCG1 (YvanCharvet et al., 2007b; Out et al., 2008). ABCA1 and ABCG1 double knockout macrophages showed apparently defective cholesterol efflux to HDL and ApoA-1 compared with either ABCA1 or ABCG1 knockout macrophages (Yvan-Charvet et al., 2007b; Out et al., 2008).

ABCG1 protein expression is mediated at both the transcriptional and posttranscriptional levels (Fig. 4). At the transcriptional level, ABCG1 shares the common regulatory pathways of gene expression with ABCA1. The ABCG1 expression is upregulated by the nuclear receptors LXR, RXR, and PPAR activated by their agonists (oxysterols, retinoids, fatty acids, or synthetic agonists) (Venkateswaran et al., 2000; Hardy et al., 2017). Upregulation of ABCG1 expression mediated by LXR agonists probably involves the presence of multiple LXR responsive elements by the ABCG1 gene promoter and likely only requires the isoform $\mathrm{LXR} \alpha$ in human macrophages (Sabol et al., 2005; Ishibashi et al., 2013). Further study suggests that LXR recruitment at the human ABCG1 locus is promoted by the G protein pathway suppressor 2 (Jakobsson et al., 2009). ABCG1 in macrophages is also transcriptionally regulated by the PPAR $\gamma$-LXR pathway (Li et al., 2004a). Retinoic acid receptor/RXR heterodimer can bind LXR responsive elements in ABCG1 promoters and transactivates ABCG1 in macrophages as well (Ayaori et al., 2012).

Other mechanisms also contribute to the stability and activity of human ABCG1. Compared with ABCA1, there is limited evidence concerning the posttranscriptional regulation of ABCG1 or protein-protein interactions involving $A B C G 1$. It was reported that calpain facilitated ABCG1 degradation by slicing ABCG1 on the cell surface (Adlakha et al., 2013). The proteasomal inhibition prevented degradation of ABCG1 and led to the accumulation of phosphorylated ABCG1 (Nagelin et al., 2009). Degradation of ABCG1 is suggested to be mediated via the ubiquitin-proteasome system-mediated non-lysosomal pathways (Nakaya et al., 2017) by the NEDD4-1 (neural precursor cell-expressed developmentally downregulated gene 4) and E3 ubiquitin ligases HUWE1 (HECT, UBA, and WWE domain containing 1 E3 ubiquitin protein ligase) (Aleidi et al., 2015). ABCG1 ubiquitination and its proteasomal degradation can be inhibited by cholesterol through interplay with a cholesterol recognition/interaction amino acid consensus (CRAC) motif located in the ABCG1 transmembrane domain (Hsieh et al., 2014; Sharpe et al., 2015).

ABCG1 protein expression was also recently investigated in relation to regulation by miRNAs. The miRNAs implicated in ABCG1 protein regulation include miRNA10b (Wang et al., 2012a), miRNA-23a-5p, miRNA-33, miRNA-128-2, miRNA-146a-5p, and miRNA-378. The inhibition of miRNA-23a-5p enhanced cholesterol efflux and decreased foam cell formation through upregulating ABCG1 expression levels. miRNA-10b repressed ABCA1 expression and downregulated cholesterol efflux from murine or human macrophages (Wang et al., 2012a). Long-term in vivo systemically delivered miRNA-23a-5p antagomir significantly increased the ABCG1 expression in the aorta of $\mathrm{ApoE}^{-/-}$mice (Yang et al., 2018a). 
miRNA-33 inhibited ABCG1 expression, thus resulting in decreasing cholesterol efflux (Rayner et al., 2010). Upregulation of miRNA-33a-5p stimulated by inflammatory cytokines (i.e., IL-6, TNF- $\alpha$ ) inhibited the ABCG1mediated cholesterol efflux from THP-1 macrophages (Mao et al., 2014). miRNA-128-2 inhibited the expression of ABCG1 directly. The administration of miRNA128-2 led to decrease in the mRNA and protein levels of ABCG1 in mice (Adlakha et al., 2013). Elevated miRNA146a-5p antagonized the increase of ABCG1 in low-dose LPS-tolerized cells ( $\mathrm{Li}$ et al., 2015b). The decrease of miRNA-378 level enhanced ABCG1-mediated macrophage cholesterol efflux to HDL by inducing ABCG1 protein expression (Wang et al., 2014a).

c. Scavenger Receptor Class B Type 1. SR-BI is an $82-\mathrm{kDa}$ integral membrane protein, which (together with lysosomal integral membrane protein-2) is a member of the CD36 superfamily of scavenger receptor proteins (Phillips, 2014). SR-BI has a hairpin-looped structure with two short N- and C-terminal transmembrane domains, two cytoplasmic tails, and a large extracellular domain (Williams et al., 1999; Meyer et al., 2013). In 1996, it was identified that SR-BI is an HDL receptor that regulates cholesterol uptake into liver cells (Acton et al., 1996). This process selectively transports the CE from mature HDL into cells without endocytosis and degradation of the HDL particles
(Acton et al., 1996). This receptor is expressed primarily in liver, where it acts in the RCT pathway (Zannis et al., 2006). In addition to promotion of delivery of HDL-C to cells, SR-BI increases the efflux of cellular cholesterol to HDL (Ji et al., 1997; Jian et al., 1998). When incubated with synthetic cholesterol-free HDL, SR-BI-transfected Chinese hamster ovary cells increased initial rates of efflux by approximately threefold compared with control cells, suggesting that SR-BI expression enhanced net cholesterol efflux regulated by HDL (Ji et al., 1997). However, compared with ABCA1 and ABCG1, the contribution of SR-BI to efflux from macrophages is small (Adorni et al., 2007). The performed studies indicated that the SR-BI is a multifunctional receptor that regulates bidirectional flux of lipids, which might be dependent on the content of cholesterol in cells (Rosenson et al., 2012).

In addition to regulating lipid metabolism, SR-BI can also mediate inflammatory responses. SR-BI interaction with HDL reduced the inflammatory response to LPS in human macrophages by markedly reducing NF$\kappa \mathrm{B}$ activation (Song et al., 2015). Furthermore, recent studies have shown that the interaction of macrophage SR-BI with apoptotic cells activated phosphoinositide 3-kinase (PI3K)/Akt signaling and induced the expression of anti-inflammatory cytokines (Tao et al., 2015a). HDL activated PI3K/Akt signaling in macrophages,

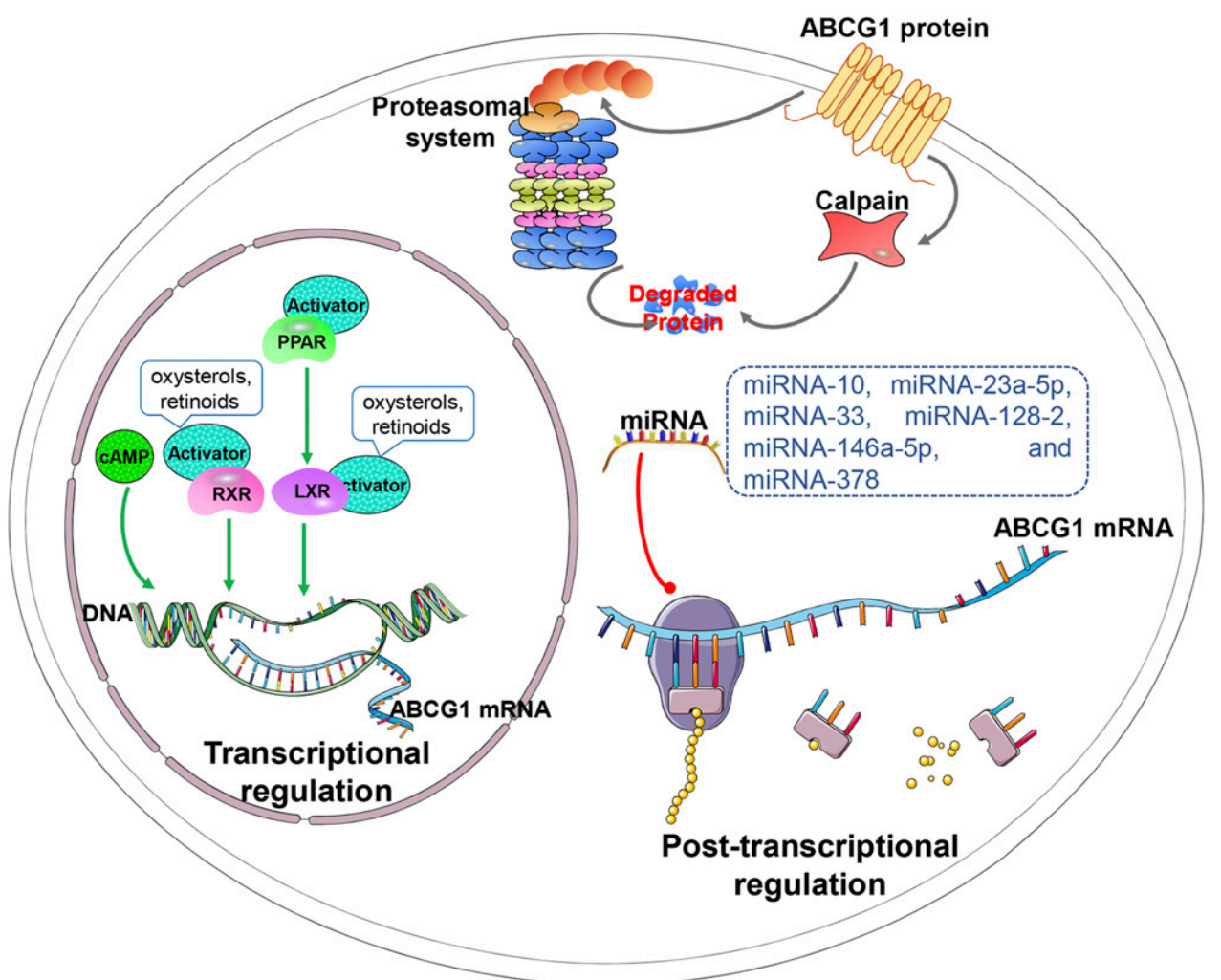

Fig. 4. Regulation of ABCG1 expression at both transcriptional and post-transcriptional levels. At the transcriptional level, ABCG1 shares common gene expression regulatory pathways with ABCA1. The ABCG1 expression is upregulated by the nuclear receptors LXR, RXR, and PPAR activated by their agonists (oxysterols, retinoids, fatty acids, or diverse synthetic agonists). At the post-transcriptional level, the protein stability of ABCA1 is governed by the proteasomal and calpain systems. Also, miRNA-10b, miRNA-23a-5p, miRNA-33, miRNA-128-2, miRNA-146a-5p, and miRNA-378 are involved in the regulation of ABCG1 protein (green arrows: upregulation; red lines: downregulation). 
which is mediated by SR-BI and involves interaction with its adaptor protein PDZK1 (PDZ domain-containing 1) and activation of sphingosine 1-phosphate receptor 1 signaling (Al-Jarallah et al., 2014). SR-BI interaction with HDL also prevents endothelial cell inflammation reaction by regulating eNOS activation and expression of the antioxidant enzyme 3-beta-hydroxysteroid-delta 24-reductase (Yuhanna et al., 2001; McGrath et al., 2009). SR-BI-mediated production of NO and 3-betahydroxysteroid-delta 24-reductase lead to alleviation of $\mathrm{TNF}-\alpha$-stimulated NF- $\kappa \mathrm{B}$ activation, resulting in the reduced expression of inflammatory monocyte adherence proteins and chemokines in endothelial cells, thereby reducing monocyte recruitment into the intima (Bess et al., 2011). Additionally, a major portion of the endothelial cell-mediated transcytosis of HDL from the apical to the basolateral side is mediated by SR-BI, suggesting that SR-BI regulated HDL transport to the subendothelium, increasing cholesterol efflux from macrophages (Rohrer et al., 2009; Vaisman et al., 2015).

In vivo studies showed that SR-BI deficiency in the bone marrow led to an accelerated atherosclerosis despite increased plasma HDL-C level, which indicated that it played a critical role in the HDL metabolism and exhibited atheroprotective effects in mice (Covey et al., 2003; Rigotti et al., 2003; Van Eck et al., 2004; Brundert et al., 2006). SR-BI overexpression in bone marrowderived cells protected against atherosclerosis in $\mathrm{LDLR}^{-/-}$ mice, as well as in $\mathrm{ApoE}^{-1-}$ mice (Covey et al., 2003; Zhang et al., 2003a). However, the physiologic effect of SR-BImediated arterial macrophage-specific cholesterol efflux in vivo is not clear (Brundert et al., 2006; Burgess et al., 2008; Yvan-Charvet et al., 2008). Some studies showed that a selective uptake of HDL-CE and the cholesterol efflux from MPMs are independent of SR-BI (Brundert et al., 2006). SR-BI in primary macrophages, although increasing cholesterol efflux in vitro, did not contribute to macrophage RCT in vivo (Wang et al., 2007a). Hepatic SR-BI modulates the changes in the composition and structure of $\mathrm{HDL}$ (El Bouhassani et al., 2011). It was indicated that SR-BI polymorphisms contributed to the functional potential of the cholesterol disposition pathway (Vergeer et al., 2011), suggesting that this receptor is involved in RCT and atherosclerosis. Human studies show that carriers of the mutation of SR-BI in humans (P297S mutation) had higher HDL-C levels, but decreased the potential for cholesterol efflux from macrophages without aggravation of atherosclerosis (Alam et al., 2001). In addition, a recent study has shown that endothelial SR-BI promoted LDL transcytosis via dedicator of cytokinesis (DOCK4) and ensuing circulating LDL entry into and retention in the artery wall to instigate atherosclerosis (Huang et al., 2019). The differential functions of SR-BI in macrophage and endothelial cells suggest the necessity to target SR-BI in a specific cell type to achieve atheroprotection.

SR-BI expression is mediated by both transcriptional and posttranscriptional mechanisms (Fig. 5).
Many molecules have been proposed to be involved in the regulation of SR-BI expression at the transcriptional level, including nuclear transcription factors [i.e., PPAR (Straus and Glass, 2007), LXR (Malerød et al., 2002), RXR, farnesoid X receptor (Malerød et al., 2005), PXR (Sporstol et al., 2005), estrogen receptors (Stangl et al., 2002), sterol regulatory element-binding proteins (SREBPs) (Lopez and McLean, 1999)] and some endogenous factors [e.g., IGF-1 (Cao et al., 2004), p38-mitogen-activated protein kinase (MAPK) cascade (Murao et al., 2008; Leiva et al., 2011]. Recent progress toward understanding the mechanisms of regulating the SR-BI expression at the transcriptional level was summarized in several reviews (Leiva et al., 2011; Shen et al., 2018a,b).

SR-BI can also be regulated posttranscriptionally, for example, by hormones such as estrogens (Zhang et al., 2007), triiodothyronine and thyromimetics (Johansson et al., 2005; Tancevski et al., 2010), insulin (Shetty et al., 2006), as well as glucagon (Nakamura et al., 2005). One important component of the posttranscriptional regulation of SR-BI is the scaffolding protein PDZK1, which regulates the SR-BI protein stability (Nakamura et al., 2005). The exact mechanisms by which the PDZK1 regulates SR-BI membrane expression remain unknown (Leiva et al., 2011). PDZK1/Na ${ }^{+} / \mathrm{H}^{+}$ exchanger regulatory factor 3 (NHERF3), NHERF1, and NHERF2 were shown to regulate hepatic SR-BI stability (Kocher et al., 2003; Hu et al., 2013b). The mechanisms involved in regulation of the SR-BI expression at the posttranscriptional level were also summarized in recently published works (Leiva et al., 2011; Shen et al., 2018a,b).

Recently, ncRNAs were also investigated in the regulation of SR-BI expression. The miRNAs, which regulate the SB-BI expression, include miRNA-96, miRNA-125a, miRNA-185, miRNA-455, and miRNA223 (Wang et al., 2013). It was shown that miRNA-96, miRNA-185, and miRNA-223 directly bound the 3 '-UTR of SR-BI mRNA to repress the level of SR-BI expression and the uptake of HDL. Furthermore, the decrease of miRNA-185 and miRNA-96 is related to the increase of SR-BI in the livers of $\mathrm{ApoE}^{-/-}$mice with a high-fat diet (HFD) (Wang et al., 2013). Another study reported that miRNA-125a and miRNA-455 negatively regulated the SR-BI expression by binding to 3'-UTR of SR-BI mRNA (Hu et al., 2012). Recent work also demonstrated that obesity induced miRNA-24 and repressed the SR-BI expression, further influencing HDL uptake, lipid metabolism, and steroid hormone synthesis (Wang et al., 2018d). However, the effect of miRNAs on the SR-BI expression and cholesterol efflux in macrophage remains to be further studied.

3. Acceptors that Mediate Macrophage Cholesterol Efflux.

a. Apolipoprotein A-1. ApoA-1 is a main protein constituent of HDL ( $\sim 70 \%$ of the HDL protein) (Phillips, 
2013). Human mature ApoA-1 (molecular weight: 28 $\mathrm{kDa}$ ) comprises a total of 243 amino acid residues. The C-terminal domain of human ApoA-1 contains 11- and 22-amino acid tandem repeats ( $\mathrm{Li}$ et al., 1988). Each repeat has an amphipathic $\alpha$-helix, which is crucial for its efficient interaction with lipids in an exchangeable manner (Segrest et al., 1992). The motifs of amphipathic $\alpha$-helices in ApoA-1 are evolutionally preserved (Bashtovyy et al., 2011). The C-terminal domain takes responsibility for the almost all ApoA-1 lipid-solubilizing property (Tanaka et al., 2008). Furthermore, the C-terminal domain performs as an intact protein, which is able to solubilize phospholipids and facilitate HDL disk formation (Lyssenko et al., 2012). The hydrophobic character of the C-terminal domain and the stability of the N-terminal helix bundle are the major factors for lipid-solubilizing and lipid-binding properties of ApoA-1 (Chistiakov et al., 2016b).

ApoA-1 regulates RCT through mediating cholesterol efflux from foam cells by interaction with the transporter ABCA1 (Mukhamedova et al., 2008). Lipidation of ApoA- 1 by cooperation with ABCA1 generates discoidal nascent HDL particles (Wang and Smith, 2014).
Since ApoA-1 is directly involved in lipid metabolism, it is considered as one of the key molecular players in the pathogenesis of atherosclerosis (Chistiakov et al., 2016b). In plasma, ApoA-1 is also involved in the esterification of FC in serum lipoproteins by stimulation of lecithin cholesterol acyltransferase (LCAT), an enzyme secreted by the liver (Zannis et al., 2006). The effect of ApoA-1 in the protection against atherosclerosis has been examined in some in vivo studies. In $\mathrm{LDLR}^{-/-}$mice, ApoA-1 deficiency resulted in aggravating atherosclerosis (Moore et al., 2003). On the contrary, human ApoA-1 overexpression and ApoA-1 infusions in mice mitigated atherosclerotic lesion formation in animal models of atherosclerosis (Duverger et al., 1996). Furthermore, mutations of ApoA-1 enhance CVD risk in humans. Most of the carriers of mutations in the ApoA-1 gene have a lower level of HDL-C and a higher risk of CVD compared with those who do not carry the mutation (Hovingh et al., 2004). Thus, ApoA-1 is widely believed as a promising target for treatment of CVD (Stoekenbroek et al., 2015). There have been some therapies to mimic ApoA-1, including full-length ApoA-1, mutated variants of ApoA-1, and ApoA-1 mimetic peptides,

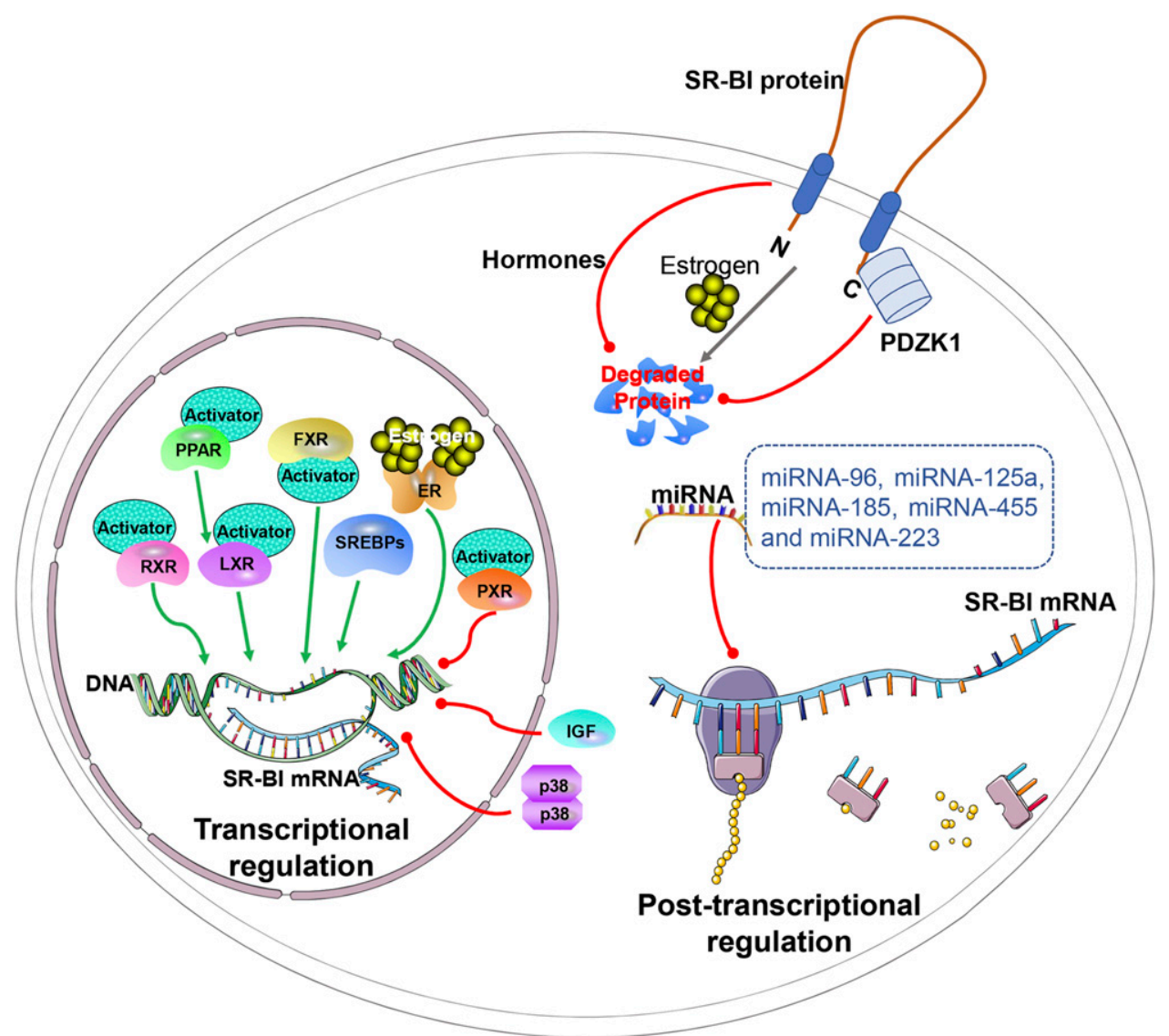

Fig. 5. Regulation of SR-BI expression at both transcriptional and post-transcriptional levels. At the transcriptional level, SR-BI protein expression is regulated by a variety of molecules, including nuclear transcription factors [such as the heterodimeric nuclear receptors PPAR, LXR, RXR, FXR (farnesoid X receptor), and PXR], estrogen receptors, sterol regulatory element-binding proteins (SREBPs), and other endogenous signaling factors (e.g., IGF-1 and p38-MAPK cascade). At the post-transcriptional level, SR-BI can be regulated by estrogens insulin and glucagon as well as other hormones [e.g., triiodothyronin (T3) and thyromimetics]. In addition, miRNA-96, miRNA-125a, miRNA-185, miRNA-455, and miRNA-223 are involved in SR-BI protein regulation. (green arrows: upregulation; red lines: downregulation). 
that were well described in several reviews (Millar and Cuchel, 2015; Stoekenbroek et al., 2015). The ApoA-1mimetic peptides (e.g., 4F, 6F, FX-5A, ATI-5261, and ETC-642) (Garber et al., 1992; Wool et al., 2008; Stoekenbroek et al., 2015) and modified ApoA-1 (Graversen et al., 2008) strongly induced the macrophage cholesterol efflux and exhibited antiatherogenic properties. In the "ApoA-1 Milano Trial," plaque thickness was significantly decreased in patients treated with ApoA-1 $1_{\text {Milano }}$ compared with the control group (Nissen et al., 2003; Nicholls et al., 2006). However, additional well-designed clinical trials are required to address the therapeutic role of ApoA-1 mimetics on CVD (Stoekenbroek et al., 2015). In addition, it remains a challenge whether increase of the ApoA-1 production or infusion can be performed effectively and safely.

The studies of regulation of ApoA-1 showed that $\operatorname{PPAR} \alpha$ was able to regulate ApoA-1 transcription. Activators of PPAR $\alpha$ (e.g., fibrate) increased HDL-C levels by promoting ApoA-1 transcription in preclinical studies (Singh et al., 2005; Fruchart, 2013). However, treatment with a PPAR $\alpha$ agonist LY518674 did not remarkably alter HDL-C levels in humans (Nissen et al., 2007). The mechanisms of the regulation of ApoA-1 expression remain to be further studied.

b. High-density Lipoprotein. In 1929, HDL was firstly discovered as a protein-rich, lipid-poor complex, which was isolated from equine serum (Kingwell et al., 2014). Later in the 1950s, HDL was also extracted from human serum as a chemical entity by ultracentrifugation (Barr et al., 1951). Among lipoprotein particles, HDL are the smaller (7-12 $\mathrm{nm}$ in diameter) but denser $(1.063-1.21 \mathrm{~g} / \mathrm{ml})$ particle due to the higher protein ratio (50\% of the content is protein) (Phillips, 2013). HDL is mainly produced by liver and intestine (Nofer et al., 2002). Nascent HDL (discoidal HDL) is formed through lipidation of ApoA-1. Discoidal HDL particles were shown to contain two ApoA-1 molecules and 140 1-palmitoyl-2oleoyl-sn-glycero-3-phosphocholine molecules (Davidson and Thompson, 2007). During more ApoA-1 incorporation to the HDL particles, conformational changes increase the $\alpha$-helix content by $40 \%$ (Koppaka et al., 1999). In human blood, the circulating HDL is predominantly presented in the form of HDL spheres. The spherical HDL particles are formed from free cholesterol-containing discoidal HDL particles by converting $\mathrm{FC}$ into $\mathrm{CE}$, which can then be internalized into the core of the HDL particles (Ferretti et al., 2006; Kuai et al., 2016). In spherical HDL, ApoA-1 plays the major role in stabilizing HDL structure and shape (Hewing et al., 2014). Spherical HDL can further internalize cholesterol from cells (which is mediated by ABCG1 and SR-BI) to form more mature and larger spherical HDL (Kennedy et al., 2005). Mature HDL transports the lipids to liver via an SR-BI-mediated endocytosis to be further metabolized (Kingwell et al., 2014).

Today, it is verified that HDL plays an important role in the lipid [cholesterol and triglycerides (TG)] transport and metabolism (Wang and Briggs, 2004). HDL particles induce the efflux of excessive lipids from cells, thus being involved in RCT from macrophages (LundKatz and Phillips, 2010). In addition to its role in the HDL-mediated macrophage cholesterol efflux, it also has anti-inflammatory, antiapoptotic, antioxidative, and vasodilatory properties, which are also protective in the cardiovascular system (Besler et al., 2012). Circulating HDL also delivers signaling lipids, vitamins, endogenous proteins, hormones, and miRNA to different organs (Kuai et al., 2016), indicating that HDL plays multiple roles in complicated intercellular communication (Vickers et al., 2011). Treatment with infusions of homologous HDL-VHDL significantly reduced the aortic surface area covered by lipid-rich lesions compared with controls (Stoekenbroek et al., 2015), and decreased the extent of preexisting lesions in a rabbit model (Badimon et al., 1990). However, recent clinical studies show that inhibitors of cholesteryl ester transfer protein (CETP) upregulated the HDL-C levels in subjects with normal or low HDL-C. Nevertheless, reduced risk of atherosclerotic diseases was not demonstrated (Schwartz et al., 2009, 2012).

Some reconstituted HDL (rHDL)-based medicines have been propelled to different stages of clinical trials (Krause and Remaley, 2013). Some rHDL products that have been evaluated in clinical trials include CSL-111, CSL-112, ETC-216, ETC-642, SRC-rHDL, and CER-001 (Kuai et al., 2016). The results of one clinical trial showed that there was no significant difference in the atheroma volume and coronary score between patients treated with rHDL CER-001 and those treated with placebo (Tardif et al., 2014). Some other studies suggest that against a background of statin treatment, there may be no clinical benefit of raising HDL-C (Schwartz et al., 2012; Niesor et al., 2015). On the contrary, preliminary clinical results at the 2013 PACE Snapshot session (ESC, Amsterdam) display that CER-001 increased RCT and might decrease the aortic atheroma volume (Goffinet et al., 2012). A clinical report also showed that most patients with elevated cardiovascular risk were treated with a statin and large meta-analyses suggest low HDL-C still represents a cardiovascular risk factor on a background of statin treatment (Baigent et al., 2005). Overall, further clinical studies are required to confirm the anti-atherosclerotic effect of rHDL.

c. Apolipoprotein E. ApoE is a soluble $34-\mathrm{kDa}$ glycoprotein, coded by three alleles (Mahley, 1988). ApoE is essential for the lipid metabolism (production, conversion, and clearance of lipoproteins) in all tissues and organs (Liehn et al., 2018) and is recognized for its ability to suppress atherosclerosis (Raffai, 2012). Although ApoE is synthesized mainly by liver (Kraft et al., 1989), many other cells or tissues are also able to synthesize ApoE, such as macrophages, adipocytes, smooth muscle cells, brain, and kidney (Driscoll and Getz, 1984; Zechner et al., 1991). The expression of 
ApoE in the macrophages has been believed to prevent atherosclerosis by inducing cholesterol efflux from foam cells (Fazio et al., 1997; Curtiss and Boisvert, 2000).

ApoE has been shown to mediate uptake of chylomicrons, very LDL (VLDL) remnants, and ApoE-containing HDL (Liehn et al., 2018), as well as to regulate myelopoiesis (Murphy et al., 2011). ApoE is also known to regulate cellular signaling through the interaction with its receptors and heparin sulfate proteoglycans and to influence several biologic effects, including macrophage plasticity, smooth muscle cell proliferation and endothelial cell activation (Curtiss and Boisvert, 2000). ApoE suppresses NF- $\kappa$ B-driven inflammation and atherosclerosis by influencing the levels of miRNA-146a in monocytes and macrophages in hyperlipidemic mice (Li et al., 2015a). ApoE inhibited the VSMC proliferation and aortic stiffening by regulating $\mathrm{p} 27$ through reducing the levels of miRNA221/222 and increasing the levels of miRNA145, respectively (Kothapalli et al., 2013). In addition, ApoE stimulated the eNOS activation and exhibited anti-inflammatory property, thus preventing neointima formation by binding to ApoE receptor 2 (ApoER2) (Ulrich et al., 2014). Additionally, there is a tight link between ApoE and neurodegenerative diseases. It was reported that the ApoE4 polymorphism is one main risk factor for the development of Alzheimer disease (AD) (Giau et al., 2015). In animal studies, $\mathrm{ApoE}^{-/-}$mice developed atherosclerotic lesions spontaneously, similar to those observed in humans, and these lesions were exacerbated when mice were fed with a HFD. Therefore, the $\mathrm{ApoE}^{-/-}$mice became one of the main animal models of atherosclerotic plaque initiation and growth in cardiovascular research (Daugherty, 2002). ApoE prevents the formation of foam cells through mediating cholesterol efflux via ABCA1/ABCG1 pathway (YvanCharvet et al., 2010b).

ApoE is mediated at both the transcriptional and posttranscriptional levels. A recent review (Kockx et al., 2018) summarized the insights regarding the regulation of ApoE production and secretion by monocytes/ macrophages, adipocytes, hepatocytes, and the central nervous system. The regulation of ApoE expression is remarkably cell, differentiation, and tissue specific. At the transcriptional level, cholesterol loading in macrophages increased transcription and secretion of ApoE and thus promoted macrophage cholesterol efflux in vitro (Kockx et al., 2012). ApoE can also be regulated by LXR at the transcriptional level (Kockx et al., 2018). At the posttranscriptional regulation, synthesized ApoE in the ER is moved via the Golgi and trans-Golgi network, during which ApoE is further glycosylated and sialylated (Kockx et al., 2018). Accumulation of excessive FC in the ER prevented the movement of ApoE from the ER to Golgi (Kockx et al., 2012). In macrophages, large ratio of $\mathrm{ApoE}$ is degraded after leaving the Golgi. Stimulation by ApoA-1 or HDL can prevent ApoE degradation and increases its secretion (Dory,
1991; Kockx et al., 2004). Adipocytes are induced to express and secrete substantial amounts of ApoE during differentiation (Zechner et al., 1991). PKA and dynamin was reported to mediate ApoE secretion in HepG2 cells (Kockx et al., 2009). The LXR-ABCA1 pathway is a common ApoE-activating pathway in astrocytes (Kockx et al., 2018). ApoE secretion by neurons in the central nervous system depends on calcium and microtubule (Dekroon and Armati, 2002). The mechanisms regulating the ApoE expression and secretion in different cell types and tissues have not been extensively studied yet.

\section{Models for Studying Foam Cell Formation}

Foam cell formation is a hallmark of atherosclerosis. Thus, numerous studies have used a variety of models to study the process of foam cell formation and to screen promising bioactive compounds targeting foam cell formation. In this section, we will discuss the models widely used for in vitro, ex vivo, and in vivo studies on both cholesterol uptake and efflux.

\section{A. Cellular Models}

So far, prevention of foam cell formation has mostly been focused on monocytes and macrophages. It was also demonstrated that different cell types present in the artery wall, such as endothelial cells, VSMCs (Maguire et al., 2019), as well as stem cells can exhibit foam cell-like characters and behavior in the growing neointima of atherosclerosis in both human beings (Daub et al., 2006) and mice (Feng et al., 2012). Thus, the cellular models for study on inhibition of foam cell formation include monocytes, macrophages (Hayden et al., 2002), endothelial cells (Constantinescu et al., 2000), VSMCs (Zhang et al., 2016b), and stem/progenitor cells (Zhang et al., 2018). At present, mouse macrophage cell types used for study on inhibition of foam cell formation include J774A.1, RAW264.7 (Li et al., 2013), P388D1 macrophages (Koren et al., 1990), primary MPMs (Sengupta et al., 2013), and bone marrowderived macrophages (BMDMs). One study has suggested that different types of mouse macrophages might not have significant difference on the cholesterol efflux assays because RAW264.7 and J774A.1 macrophages have highly correlated assay values (Li et al., 2013). Human macrophage cell types used in foam cell formation studies include phorbol 12-myristate 13-acetate (PMA)-stimulated THP-1-derived macrophages, HL-60 cell-derived macrophages and primary human monocytederived macrophages (HMDMs) (Kosaka et al., 2001).

1. Models for Studying Cholesterol Uptake. Lipid uptake can be assessed by neutral lipid-targeting lysochrome Oil red O staining, Nile red staining, 1,1'dioctadecyl-3,3,3'3'-tetra-methylindocyanide percholorate (DiI)-labeled oxLDL (DiI-oxLDL), DiI-LDL, and DiI-acLDL (Xu et al., 2010), NBD-cholesterol, as 
well as radiolabeled LDL or derived LDL (e.g., ${ }^{125}$ I-LDL, ${ }^{131}$ I-LDL) (Suits et al., 1989).

For Oil red O staining, macrophages are incubated with oxLDL with or without ACAT1 inhibitor in the presence or absence of tested natural products. The cells are then fixed with $4 \%$ formaldehyde and stained with Oil red $\mathrm{O}$ solution to identify lipid droplets containing $\mathrm{CE}$ to assess the amount of lipid inside the cell (lipid uptake) (Kosaka et al., 2001). Lipid accumulation in cells can be assessed by a microscope and separated into different grades according to the intracellular lipid droplet-occupied area. The percentage of foam cells is calculated by counting the total cell and foam cell numbers (Kosaka et al., 2001; Das et al., 2013). Oil red $\mathrm{O}$-stained lipid droplets can also be assessed spectrophotometrically at $518 \mathrm{~nm}$ after lysis of the cells (Guo et al., 2006). Nile red, 9-diethylamino-5H-benzo[alpha] phenoxazine-5-one, is also an important staining method for the determination of intracellular lipid droplets by using fluorescent microscopy and flow cytofluorometry (Greenspan et al., 1985). The increase in fluorescence intensity can be quantitatively determined by the interactive laser cytometer (Koren et al., 1990). Its staining process and application are almost the same with Oil red $\mathrm{O}$ for staining lipids. In addition, fluorescent NBD-cholesterol [22-( $N$-(7-nitrobenz-2-oxa1,3-diazol-4-yl)amino)-23,24-bisnor-5-cholen-3 $\beta$-Ol)] is also used to assess cholesterol uptake (Frolov et al., 2000; Sengupta et al., 2013). Cells are incubated with NBD-cholesterol and then lysed by adding methanol. Supernatant of cells lysate is used to measure fluorescence intensity by using fluorescence plate reader at emission spectra of $535 \mathrm{~nm}$ upon excitation at $475 \mathrm{~nm}$ (Frolov et al., 2000; Sengupta et al., 2013).

Staining with Oil Red O requires fixation of cells and staining in the presence of organic solvents (e.g., isopropanol) and is, therefore, limited to the analysis of dead cells (Majka et al., 2014). Water soluble Nile red can be used to stain both live and dead cells. However, its emission maximum at $528 \mathrm{~nm}$ may overlap with green (FITC, GFP) and yellow-orange fluorophores in flow cytometry (Majka et al., 2014). In recent years, a big progress has been made to develop new LD-specific probes [e.g., LipidTOX stains (Majka et al., 2014)], which are discussed in a recent review (Fam et al., 2018).

For cellular uptake of DiI-oxLDL, DiI-LDL, or DiIacLDL, cells are incubated with them and then lysed (Teupser et al., 1996; Xu et al., 2010). Fluorescent intensity of the cell lysates is detected by using a microtiter plate reader with excitation-emission set at 520 and $580 \mathrm{~nm}$, respectively (Teupser et al., 1996). DiIoxLDL, DiI-LDL, or DiI-acLDL uptake in macrophages can also be evaluated by confocal microscopy and flow cytometry as described previously (Xu et al., 2010). For cellular uptake of radiolabeled LDL or derived LDL, cells are incubated with modified lipoproteins and then lysed in $\mathrm{NaOH}$ (Selmer et al., 1997). Internalized LDL is determined by measuring the $\mathrm{NaOH}$ solution in the gamma-counter (Selmer et al., 1997). In addition, foam cell formation can be indirectly evaluated by measuring the total cholesterol (TC) via using cholesterol/CE quantification kit (Das et al., 2013).

2. Models for Studying Cholesterol Efflux. One cause for foam cell formation is the inability of cells to export cholesterol to a sufficient extent (Hansson, 2005; Hansson et al., 2006), which can be assessed by quantitating the rate of cholesterol efflux from the cells. Cholesterol efflux assay is usually used to assess the influence of natural products on plasma acceptorinduced cholesterol efflux from cells (Low et al., 2012). Dr. Rothblat's group (Rothblat et al., 1999) pioneered methodologies allowing the assessment of the capacity of human serum- or HDL-induced cholesterol efflux. In general, first of all, cells are loaded with labeled lipids to form foam cells and then incubated in serum-free medium to balance labeled cholesterol in cholesterol pools in cells (de la Llera-Moya et al., 2010; Khera et al., 2011), which can be combined with treatment or stimulation of tested natural products. Then the cells are incubated with acceptors to induce cholesterol efflux. The efflux of labeled lipids from the cells can be quantified (Low et al., 2012). In addition, some studies used ACAT inhibitor to prevent re-esterification of FC to CE (de la Llera-Moya et al., 2010; Khera et al., 2011) when loading lipids to cells or other steps (e.g., equilibration).

a. Loading cells with labeled lipids to form foam cells. The conversion of macrophages to foam cells by the accumulation of modified LDL is a critical step in atherosclerosis development. One of the widely used techniques to form the foam cell model is by incubation macrophages with radioactive ${ }^{3} \mathrm{H}$-cholesterol-labeled modified LDL, especially acLDL or oxLDL (Asztalos et al., 2005; Singh et al., 2009). This method mimics the critical pathologic step (uptake of modified LDL by macrophages) in the atherosclerosis development. However, the methods of labeling acLDL with radioactive or fluorescent tags have some flaws (Sengupta et al., 2013). For example, LDL is inherently unstable and with limited shelf life, and is easy to be oxidized during the long-lasting isolation processes (Parthasarathy et al., 1999). Isolation and acetylation of LDL can also differ qualitatively between different preparations, which might offer inconsistent information regarding foam cell formation (Sengupta et al., 2013).

In recent time, a novel approach to load lipids to form foam cells has been adopted for high-throughput macrophage cholesterol efflux assay (Khera et al., 2011; Li et al., 2013). Radio-labeled cholesterol $\left({ }^{3} \mathrm{H}\right.$-cholesterol, and ${ }^{14} \mathrm{C}$-cholesterol) to load macrophages was used to study cholesterol efflux in the presence of serum from human subjects (Khera et al., 2011; Li et al., 2013). For example, J774A.1 cells were plated and labeled with 
$2 \mu \mathrm{Ci}$ of ${ }^{3} \mathrm{H}$-cholesterol per milliliter (Khera et al., 2011). In fact, the basic challenge to incorporate cholesterol in cells is its high hydrophobicity (Sengupta et al., 2013). A recent study reported a biologically relevant detergent compound, lysophosphatidylcholine, form mixed micelles with cholesterol or $\mathrm{CE}$, incubation with which could efficiently generate macrophage foam cells (Sengupta et al., 2013). Further results indicated that such micelles were quite stable at $4^{\circ} \mathrm{C}$ and maintained the solubilized cholesterol at $4^{\circ} \mathrm{C}$ for at least 1 month (Sengupta et al., 2013), suggesting this technique could be less timeconsuming and thus highly reproducible. It is worth noting that there are some studies using cholesterolmethyl- $\beta$-cyclodextrin, which significantly increases cholesterol solubility in water, to load cells with cholesterol to successfully form foam cells (Wang et al., 2018a,b,d). In this method, labeled cholesterol is added to serumcontaining media. It is supposed that the labeled cholesterol is incorporated into lipoproteins in serum, which are further taken up by cells. So, it is necessary to allow sufficient time (24-48 hours) for uptake of lipoproteins (Low et al., 2012).

In recent years, fluorescently labeled cholesterol types (e.g., NBD-cholesterol, the fluorophore Pennsylvania green $/ \mathrm{N}$-alkyl-3b-cholesterylamine-derived molecular probe [F-Ch), the dipyrromethene boron difluoride (BODIPY)-cholesterol] have become an important tool to study cholesterol metabolism including cholesterol efflux (Frolov et al., 2000; Sankaranarayanan et al., 2011; Zhang et al., 2011), because they closely mimic the properties of cholesterol regarding metabolism and intracellular trafficking (Atshaves et al., 2000). In fact, other studies indicated that NBD-cholesterol has a higher aqueous solubility compared with cholesterol, and might not really represent the uptake and efflux of cholesterol (Atshaves et al., 2000). However, a report showed that the influence of higher solubility of NBD-cholesterol on cholesterol uptake and efflux was ruled out by using ${ }^{3} \mathrm{H}$-cholesterol, a common used cholesterol analog (Sengupta et al., 2013). On the contrary, it was reported that the functional property of fluorescent sterol analogs was not closely similar with that of cholesterol (Frolov et al., 2000). F-Ch is also a recently reported fluorescent mimic of cholesterol, which is structurally similar to other cholesterylamine conjugates (Zhang et al., 2011). It was demonstrated that cholesterol efflux of this fluorescent cholesterol mimic is similar compared with ${ }^{3} \mathrm{H}$-cholesterol (Zhang et al., 2011). However, the extent of export quantified using F-Ch was somewhat smaller than that of ${ }^{3} \mathrm{H}$-cholesterol, which may be due to the molecular weight of F-Ch, which is over twice that of cholesterol, and it accumulates in early endosomes that might affect its rate of export (Zhang et al., 2011). It was demonstrated that BODIPY-cholesterol efflux is significantly associated with ${ }^{3} \mathrm{H}$-cholesterol efflux when apoB-depleted sera and pre $\beta 1-\mathrm{HDL}$ were used but not total HDL-C in
J774A.1 macrophages, suggesting that the efflux of BODIPY-cholesterol from cells was regulated primarily by ABCA1 (Sankaranarayanan et al., 2011). It is important to recognize that fluorescent cholesterol cannot completely mimic the behavior of cholesterol, thus one should choose suitable labeled cholesterol according to scientific aims.

b. Inducing cholesterol efflux to extracellular acceptors and quantification. After being loaded with lipids to form foam cells, cells are incubated in serum-free medium to balance labeled cholesterol in cholesterol pools and treated or stimulated by tested natural products. After stimulation of tested natural products, macrophages are exposed to cholesterol acceptors for several hours (2-8 hours), allowing resultant cholesterol efflux from the foam cells (Rothblat et al., 1999). In general, the time of incubation with acceptors should not be very long. Long incubation time (such as 24 hours) would reflect a state of equilibrium and therefore does not reflect the rate of cholesterol efflux (Low et al., 2012). Radioactive and fluorescent cholesterol are quantified by liquid scintillation counting (Khera et al., 2011) and fluorescence, respectively (Atshaves et al., 2000).

Numerous studies have showed that various cholesterol acceptors, ranging from cyclodextrins to serum, stimulate cholesterol efflux from macrophages (Rothblat et al., 1999). The common cholesterol acceptors include cyclodextrin $(\sim 200 \mu \mathrm{g} / \mathrm{ml})$, whole serum $(\sim 1 \%$ to $2 \%)$, plasma ( $\sim 1 \%$ to $2 \%)$, apoB-depleted serum $(\sim 1 \%$ to $2 \%)$, HDL $(\sim 20 \mu \mathrm{g} / \mathrm{ml})$, and ApoA1 $(\sim 10 \mu \mathrm{g} / \mathrm{ml})$ (Khera et al., 2011; Li et al., 2013; Wang et al., 2018a). ApoB-depleted serum, also called HDL fraction, can be obtained after removal of ApoB particles using polyethylene glycol (molecular weight 8000) (de la Llera-Moya et al., 2010). It was reported that this method yielded recovery of more than $97 \%$ of ApoA1-containing HDL particles and less than $2 \%$ of ApoB-containing LDL and VLDL particles (Khera et al., 2011).

For the quantification of exported radioactive cholesterol, the relative cholesterol efflux ratio can be calculated as the radiolabeling counts in the medium divided by total radiolabeling counts (medium and cells) in samples ( $\mathrm{Li}$ et al., 2013). To validate the methods to measure cholesterol efflux, some additional studies are needed to be considered, such as relationship between cholesterol efflux capacity duplicates and stability of cholesterol efflux assay over time (Khera et al., 2011). Some studies used a pooled serum control to correct for plate to plate and day to day variations by normalizing the data to this pooled value in subsequent analyses (Khera et al., 2011; Li et al., 2013).

For NBD-cholesterol efflux measurement, confocal microscopy can be used to analyze HDL-mediated NBDcholesterol efflux in living cells as previously described (Atshaves et al., 2000). Briefly, after loading, the cells were placed in serum-free medium with tested natural products and then HDL was added to mediate cholesterol 
efflux. A medial section passing through cells was chosen, and the fluorescence intensity of NBD-cholesterol in the total area of each cell was assessed over time and used to calculate NBD-cholesterol efflux from the cells (Atshaves et al., 2000). Since only little ( $\sim 8 \%)$ NBD-cholesterol was esterified at 24 hours (Frolov et al., 2000), the ACAT inhibitor was not applied in the fluorescence experiments. The cholesterol efflux assay has several limitations (Khera and Rader, 2013). The cell-based assays require numerous efforts to standardize and rarely enter clinical studies. In addition, this method evaluates only one step of the RCT pathway.

\section{B. Animal Models for Studying Foam Cell Formation}

The knowledge on development of atherosclerosis has been greatly deepened by studies in various animal models, including rodents, rabbits, pigs, and nonhuman primates (Tamminen et al., 1999). Mouse and rabbit models have been most broadly used (Emini Veseli et al., 2017). During the past decades, knowledge about the molecular mechanisms of development of atherogenesis has been largely improved by studies performed on transgenic and gene-targeted mice (Smithies and Maeda, 1995), including $\mathrm{ApoE}^{-/-}, \mathrm{LDLR}^{-/-}$, ApoE/ LDLR double-knockout, ApoE*3-Leiden, pro-protein convertase subtilisin/kexin type 9 (PCSK9)-adeno associated virus, and $\mathrm{ApoE}^{-/-}$fibrillin-1 mutant $\left(\mathrm{ApoE}^{-/-} \mathrm{Fbn}^{\mathrm{C} 1039 \mathrm{G}+/-}\right)$ mice (Emini Veseli et al., 2017; Maguire et al., 2019). These animal models of atherosclerosis were well detailed by several recent reviews (Getz and Reardon, 2012; Emini Veseli et al., 2017; Lee et al., 2017). It is worth noting that a reliable mouse model for lesion rupture has been developed, $\mathrm{ApoE}^{-/-} \mathrm{Fbn} 1^{\mathrm{C} 1039 \mathrm{G}+/-}$ mice (Emini Veseli et al., 2017). All these mouse models, as well as rabbit models, provide a good platform to evaluate the effect of natural products on foam cell formation in vivo and to search for promising drug candidates for treatment of atherosclerosis (Getz and Reardon, 2012; Hilgendorf and Swirski, 2012). However, it should be pointed out that studies in mice have limited suitability because of significant species differences between mice and humans (Lusis, 2000). In addition, these animal models require a genetic variation of the cholesterol processing capacity, which is coupled with extreme changes in diet (e.g., Western-type diet) (Meir and Leitersdorf, 2004; Getz and Reardon, 2012). Moreover, different monocyte biology between humans and mice renders the study on the questions regarding macrophage foam cells to be more difficult (Hilgendorf and Swirski, 2012; Angelovich et al., 2017).

Some studies established the in vivo assay of macrophage RCT, which traced movement of radiolabeled cholesterol or acLDL that originated from peripheral cholesterol-enriched macrophage cells into the blood stream and subsequent fecal elimination (Zhang et al., 2003b; Rothblat and Phillips, 2010; Weibel et al., 2011).
In this method, after treatment with tested natural products, recipient mice were injected with ${ }^{3} \mathrm{H}$-cholesterol-loaded J774A.1 macrophages intraperitoneally (Zhang et al., 2003b). Transport of ${ }^{3} \mathrm{H}$-cholesterol from the macrophages into blood, liver, and bile, as well as elimination in the feces, was measured at 48 hours after injection (Zhang et al., 2003b). In this model, overexpression of ApoA1 promoted macrophage RCT compared with control mice (Zhang et al., 2003b). Although this method is believed to be a sensitive approach to monitor cholesterol movement from macrophages in vivo, there are some critical questions (Weibel et al., 2011). For example, it is not possible to monitor cellular cholesterol homeostasis by this method (Weibel et al., 2011). One study developed a novel method employing hollow fibers to re-collect the macrophage-derived foam cells at the end of the in vivo RCT experiments, allowing quantitative analysis of the changes in cholesterol mass in foam cells for the first time (Weibel et al., 2011). Simply, cholesterol-enriched peritoneal macrophages were entrapped in semipermeable hollow fibers and implanted into the peritoneum of mice. Twenty-four hours after implantation, the fibers were removed from the peritoneum, which allowed for complete re-collection of these macrophages for quantification of changes of cellular cholesterol and protein (Weibel et al., 2011). Furthermore, it was demonstrated that the cholesterol content was increased when this experiment was performed in LDLR/apobec double knockout mice (Weibel et al., 2011). So far, this method has not yet been used to evaluate the effect of natural products on changes of cellular cholesterol.

The measurement of foam cell formation is of primary interest as a critical end-point analysis for assessing the influence of natural products on atherosclerosis development in vivo (Xu et al., 2010). Atherosclerotic lesions in in vivo studies are traditionally quantified by staining lesions en face in the whole aorta or aortic cross-section from the proximal aorta and the innominate artery with Oil red O or Sudan IV to identify lipid droplets containing CE (Kobayashi et al., 2004), followed by computer-assisted image analysis (Paigen et al., 1987; Teupser et al., 2003; Xu et al., 2010; Maganto-Garcia et al., 2012). For en face preparations of the aorta, the aortic tree is fixed and opened longitudinally, from the heart to the iliac arteries, while still linked to the heart and main branching arteries in the body, and "pinned out." After fixation and rinse, the aortas are stained with Oil red O or Sudan IV and photographed for quantification of atherosclerotic lesions (Kobayashi et al., 2004). En face lesion area of the aorta is quantified relative to its surface area. The detailed protocol for this method is reviewed elsewhere (Maganto-Garcia et al., 2012). For cross-sectional analysis of the aorta, sections of the proximal aorta are obtained sequentially, starting at the aortic valve (Kobayashi et al., 2004). Sections are stained with Oil 
red $\mathrm{O}$ and then counterstained with hematoxylin (Plump et al., 1992). Several sections are used to quantify lesion areas by using appropriate software (e.g., Image Pro Plus) (Kobayashi et al., 2004). It was noted that lesions in innominate artery are normally more advanced and larger than that in other areas (Bentzon et al., 2001; Reardon et al., 2001). For crosssectional analysis of the innominate artery (also named brachiocephalic artery), the Y-shaped piece of innominate artery is sectioned distally (Teupser et al., 2003). Sections are stained with hematoxylin and eosin and used for quantification of lesion areas (Kobayashi et al., 2004). Atherosclerotic lesions in luminal and the internal elastic lamina are evaluated in equidistant Oil red O-stained sections (Teupser et al., 2003). The detailed protocol for this method is reviewed elsewhere (Maganto-Garcia et al., 2012).

\section{Natural Products Targeting Foam Cell Formation in Atherosclerosis}

Natural products from plants, fungi, and marine sources (Dias et al., 2012) represent a rich source for the discovery of new drug leads, since only about $6 \%$ of the existing higher plants have been investigated pharmacologically (Cragg and Newman, 2013). In the past several decades, an increasing number of publications have reported that diverse natural products are able to modulate foam cell formation in atherosclerosis, including naturally occurring flavonoids, terpenoids, phenolic compounds, phenylpropanoids, alkaloids, steroids, fatty acids, amino acids, carbohydrates, and among others (Table 1). In this section, we review some important natural products targeting foam cell formation in atherosclerosis.

\section{A. Flavonoids}

Flavonoids are a class of secondary metabolites from plant and fungus, possessing 15 carbon atoms and divided into 6 major subclasses, namely anthocyanidins, flavans, flavanones, flavononols, anthoxanthins, and isoflavonoids. Flavononols and anthoxanthins (particularly the group of flavones) are the two main classes and most widespread in the human diet. They have been shown to exhibit antiallergic (Yamamoto and Gaynor, 2001), anti-inflammatory (Yamamoto and Gaynor, 2001; Cazarolli et al., 2008), antioxidative (Cazarolli et al., 2008), antibacterial (Cushnie and Lamb, 2011), anticancer (de Sousa et al., 2007), and antiatherosclerotic activities through different mechanisms in vitro and in vivo. The biologic effects of flavonoids appear to be related to their ability to regulate diverse cell-signaling cascades. In this section, we review the studies focusing on natural products belonging to the class of flavonoids, which have displayed influence on macrophage foam cell formation.
1. Alpinetin. Alpinetin (also known as 7-hydroxy-5methoxyflavanone) is the main bioactive component in the seeds of Alpinia katsumadai Hayata. Alpinetin is also present in Amomum subulatum, Scutellaria rivularis, and plants from the ginger family, such as turmeric and cardamom (He et al., 2005, 2006). Recent studies have shown that alpinetin exerted multiple pharmacological properties, such as antitumor, antiinflammatory, and antioxidative activities, and inhibition of platelet aggregation (Ramírez-Tortosa et al., 1999; Tang et al., 2012). It was shown that alpinetin $(50-150 \mu \mathrm{g} / \mathrm{ml})$ promoted cholesterol efflux and elevated the expression of PPAR $\gamma$ and LXR $\alpha$ in oxLDLstimulated THP-1 macrophages and HMDMs. It also inhibited lipid accumulation by enhancing the expression of ABCA1 and ABCG1, suggesting that alpinetin may inhibit foam cell formation by targeting the PPAR $\gamma / \mathrm{LXR} \alpha / \mathrm{ABCA} 1 / \mathrm{ABCG} 1$ pathway (Jiang et al., 2015). Furthermore, it is also reported that alpinetin $(50-200 \mu \mathrm{g} / \mathrm{ml})$ had an anti-inflammatory effect by inhibiting the TNF- $\alpha$, IL-6, and IL- $1 \beta$ expression in LPS-stimulated human macrophages (Hu et al., 2013a). While TNF- $\alpha$ reduced both ABCA1 and LXR $\alpha$ expression and suppressed the cholesterol efflux from monocytes (Voloshyna et al., 2014), it is possible that alpinetin may reverse the suppression of cholesterol efflux proteins ABCA1/ABCG1 and abolish the formation of foam cells by suppressing TNF- $\alpha$ expression.

2. Anthocyanin. Anthocyanins (ACs) are a class of water-soluble natural pigments widely present in darkcolored fruits and foods, such as purple sweet potato, grape, blueberry, black rice, and black soybean. In general, ACs comprise six important types of pigments, including geranium, cyanidin, delphinidin, peony, morning glory, and mallow pigment. Both ACs and anthocyanidins (the sugar-free counterparts of ACs), have shown various biologic activities. For example, ACs regulate blood lipids by decreasing the levels of TG, TC, and LDL-cholesterol (LDL-C), suggesting that ACs may have positive effects on CVD (Wallace et al., 2016). Anthocyanin mixture also inhibited the inflammatory response in hypercholesterolemic patients by decreasing the levels of serum high sensitivity C-reactive protein (hs-CRP) (Zhu et al., 2013).

Two bioactive ACs, cyanidin-3-glucoside $(10 \mu \mathrm{M})$ and peonidin-3-glucoside $(100 \mu \mathrm{M})$, significantly increased the macrophage cholesterol efflux in MPMs through the PPAR $\gamma$-LXR $\alpha$-ABCA1 pathway (Xia et al., 2005). Moreover, in THP-1-derived macrophages, AC-rich fraction extracted from the wild blueberry (Vaccinium angustifolium) powder reduced lipid accumulation (Del Bo' et al., 2016). Another similar observation was recorded in human kidney 2 cells, cyanidin-3- $O-\beta$-glucoside chloride, or cyanidin chloride $(50 \mu \mathrm{M})$ inhibited the high-glucose-induced cholesterol accumulation and inflammation by activating $\mathrm{LXR} \alpha$ pathway, and activated the PPAR $\alpha$-LXR $\alpha$-ABCA1-dependent cholesterol efflux (Du et al., 2015). 
TABLE 1

Natural products targeting foam cell formation in atherosclerosis

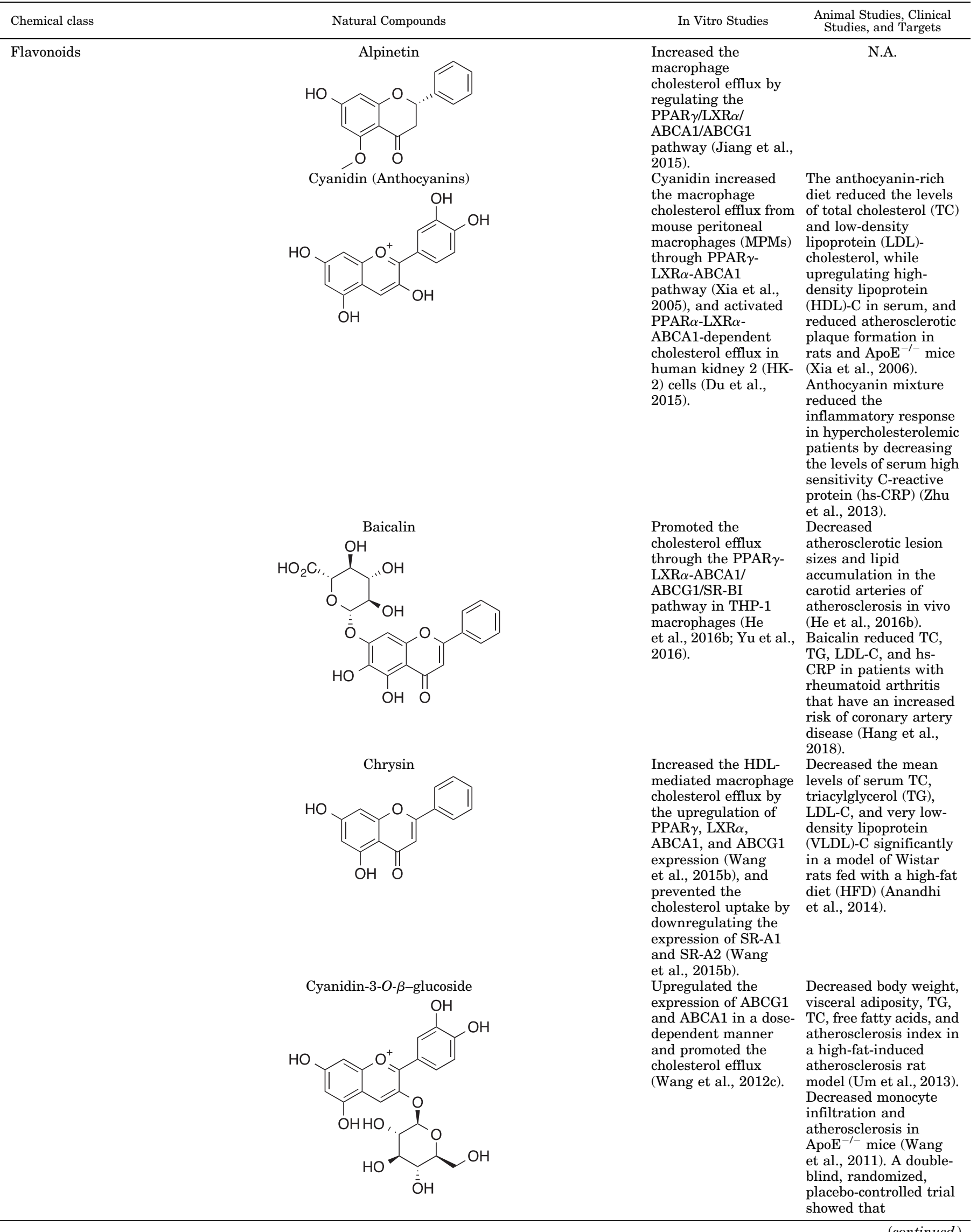




In Vitro Studies

anthocyanin

consumption increased

HDL-C, decreased

LDL-C, and promoted cellular cholesterol efflux in

hyperlipidemia

patients. Cyanidin 3-O-

$\beta$-glucosides revealed to inhibit CETP (Qin

et al., 2009).

Induced paraoxonase- Decreased the serum 1 (PON-1) activity, which may regulate cholesterol efflux by

stimulating the cholesterol, increased<smiles>O=c1c(-c2ccc(O)cc2)coc2cc(O)ccc12</smiles>

PPAR $\gamma / \mathrm{LXR} \alpha / \mathrm{ABCA} 1$ pathway (Gao et al., 2008; Robb and Stuart, 2014). triglyceride (TG) level in the male middleaged rats with HFD (Sosić-Jurjević et al., 2007), reduced plasma VLDL, LDL-C, and TG concentrations, while increased the HDL-C levels in a rheumatoid arthritis rat model (Ahmad et al., 2016). Daidzein was reported to be an inhibitor of HMG-CoA reductase, ACAT1, and ACAT2 (Borradaile et al., 2002; Sung et al., 2004).

Ellagic acid<smiles>O=c1oc2c(O)c(O)cc3c(=O)oc4c(O)c(O)cc1c4c23</smiles>

Hesperetin<smiles>COc1ccc([C@H]2CC(=O)c3c(O)cc(O)cc3O2)cc1O</smiles><smiles>COc1ccc(-c2oc3c(CC=C(C)C)c(O[C@H]4C[C@H](CO)C[C@H](O)[C@H]4O)cc(O)c3c(=O)c2O[C@@H]2O[C@H](C)[C@@H](O)[C@H](O)[C@H]2O)cc1</smiles>

Stimulated cholesterol efflux by promoting the expression of ABCA1 and SR-BI and upregulating PPAR $\gamma$ and LXR $\alpha$ (Park et al., 2011), inhibited macrophage lipid accumulation by decreasing the expression of CD36 (Aviram et al., 2008).

Increased activities of ABCA1 promoter and LXR enhancer, the expression of ABCA1, and consequently upregulated the ApoA-1-mediated cholesterol efflux (Iio et al., 2012).

Inhibited cholesterol intake and foam cell formation by the reduced expression of CD36 and upregulated SR-BI expression through p38 MAPK pathway (Yang et al., 2015).
Decreased the level of

TC and TG and upregulated the expression of LXR $\alpha$, $\operatorname{PPAR} \alpha, \operatorname{PPAR} \gamma$, and their downstream gene ABCA1 in the high-fat fed hamster model (Aviram et al., 2008). Reduced atherosclerotic lesions in $\mathrm{ApoE}^{-1-}$ mice (Aviram et al., 2008).

Reduced foam cell formation in plaques by enhancing the

expression of ABCA1 (Iio et al., 2012). Reduced plasma TC level, endothelial dysfunction, macrophage infiltration, and atherosclerotic lesion in $\mathrm{ApoE}^{-/-}$mice (Sugasawa et al., 2019). Decreased the concentrations of TC TG, and LDL-C in models of normal rats (Hu et al., 2016b) and $\mathrm{ApoE}^{-/-}$mice with HFD (Xiao et al., 2017). 
TABLE 1-Continued

\begin{tabular}{|c|c|c|c|}
\hline \multirow[t]{2}{*}{ Chemical class } & Natural Compounds & In Vitro Studies & $\begin{array}{l}\text { Animal Studies, Clinical } \\
\text { Studies, and Targets }\end{array}$ \\
\hline & Iristectorigenin $\mathrm{B}$ & $\begin{array}{l}\text { Acted as a novel LXR } \\
\text { modulator that } \\
\text { increases ABCA1 and } \\
\text { ABCG1 expression in } \\
\text { RAW } 264.7 \\
\text { macrophage (Jun } \\
\text { et al., 2012). }\end{array}$ & N.A. \\
\hline & Pratensein & $\begin{array}{l}\text { Upregulated the CLA- } \\
1 \text { expression, a human } \\
\text { homolog of SR-BI, } \\
\text { may play a potential } \\
\text { role in cholesterol } \\
\text { efflux to HDL in vitro } \\
\text { (Yang et al., 2007, } \\
2009 \text { ). }\end{array}$ & $\begin{array}{l}\text { One clinical trial } \\
\text { showed that red clover } \\
\text { isoflavones } \\
\text { significantly reduced } \\
\text { the incidence of } \\
\text { arteriosclerosis } \\
\text { (Gordon, 2003). }\end{array}$ \\
\hline & Quercetin & $\begin{array}{l}\text { Promoted ABCA1- } \\
\text { mediated cholesterol } \\
\text { efflux through the } \\
\text { pathways involving } \\
\text { miRNA-7, STK11, and } \\
\text { the AMPK/PPAR } \gamma / \\
\text { LXR } \alpha \text { /ABCA1 cascade } \\
\text { (Li et al., 2017a) } \\
\text { suppressed lipid } \\
\text { deposition by } \\
\text { downregulating the } \\
\text { expression of CD36 } \\
\text { (Zhang et al., 2015). } \\
\text { Enhanced ApoA-1- } \\
\text { mediated cholesterol } \\
\text { efflux, induced } \\
\text { ABCA1 expression, } \\
\text { and increased the } \\
\text { expression of PPAR } \gamma \\
\text { in THP-1-derived } \\
\text { foam cells (Sun et al., } \\
\text { 2015) and LXR } \alpha \\
\text { activity (Lee et al., } \\
\text { 2013). }\end{array}$ & $\begin{array}{l}\text { Quercetin inhibits } \\
\text { atherosclerosis by } \\
\text { promoting ABCA1- and } \\
\text { ABCG1-dependent } \\
\text { reverse cholesterol } \\
\text { transport in ApoE }{ }^{-/-} \\
\text {mice (Cui et al., 2017). }\end{array}$ \\
\hline & ssilybin A (Silymari & $\begin{array}{l}\text { Isosilybin A promoted } \\
\text { cholesterol efflux from } \\
\text { THP-1 macrophages } \\
\text { by activating PPAR } \\
\text { (Wang et al., 2015a). }\end{array}$ & $\begin{array}{l}\text { Supplementation of } \\
\text { silybin in food } \\
\text { decreased serum level } \\
\text { of TC, TG, VLDL-C, } \\
\text { LDL-C and increased } \\
\text { HDL-C in model of } \\
\text { hypercholesterolemic } \\
\text { rats (Wang et al., 2005). } \\
\text { Furthermore, silybin } \\
\text { reduced the formation } \\
\text { of atherosclerotic } \\
\text { plaque } \\
\text { (Gobalakrishnan et al., } \\
\text { 2016). }\end{array}$ \\
\hline & Wogonin & $\begin{array}{l}\text { Enhanced cholesterol } \\
\text { efflux through } \\
\text { increasing the ABCA1 } \\
\text { protein expression } \\
\text { (Chen et al., 2011) and } \\
\text { decreasing } \\
\text { phosphorylated level } \\
\text { of ABCA1 protein. }\end{array}$ & $\begin{array}{l}\text { Wogonin ameliorated } \\
\text { hyperglycemia and } \\
\text { dyslipidemia in } \mathrm{db} / \mathrm{db} \\
\text { mice via PPAR } \alpha \\
\text { activation (Bak et al., } \\
\text { 2014). }\end{array}$ \\
\hline Terpenoids & Astaxanthin & $\begin{array}{l}\text { Downregulated the } \\
\text { expression of SR-A } \\
\text { and CD36, which is } \\
\text { relevant to uptake of } \\
\text { cholesterol in THP-1 } \\
\text { macrophages } \\
\text { (Yoshida et al., 2010). } \\
\text { Promoted ABCA1/G1 } \\
\text { expression, resulting } \\
\text { in increased ApoA-1/ }\end{array}$ & $\begin{array}{l}\text { A randomized, placebo- } \\
\text { controlled clinical study } \\
\text { in humans with mild } \\
\text { hyperlipidemia } \\
\text { displayed that } \\
\text { astaxanthin } \\
\text { administration } \\
\text { significantly increased } \\
\text { HDL-C (Yoshida et al., } \\
\text { 2010). Astaxanthin also }\end{array}$ \\
\hline
\end{tabular}


TABLE 1-Continued

Natural Compounds

Capsanthin

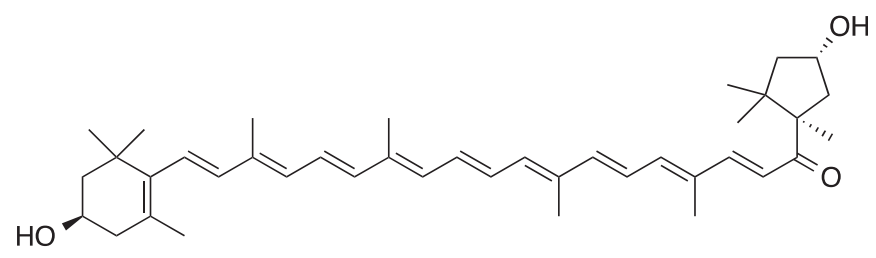

$\beta$-Carotene ( $\beta$-carotene isomers all-trans- $\beta$-carotene (all-trans- $\beta \mathrm{c}$ ), and 9-cis- $\beta$-carotene $(9-$ cis- $\beta \mathrm{c}))$<smiles>CC1=C(/C=C/C(C)=C/C=C/C(C)=C/C=C/C=C(C)/C=C/C=C(C)/C=C/C2=C(C)CCCC2(C)C)C(C)(C)CCC1</smiles>

9-cis retinoic acid (9-cis-RA, Retinoids)<smiles>CC1=C(/C=C/C(C)=C/C=C/C(C)=C/CO)C(C)(C)CCC1</smiles>

In Vitro Studies

HDL-mediated cholesterol efflux from infiltration, and plaque RAW264.7 cells via an LXR-independent manner

(Ramírez-Tortosa et al., 1999; Iizuka et al., 2012).

N.A.

Suppressed cellular cholesterol synthesis by inhibiting cellular HMG-CoA reductase activity in J774

macrophages (Fuhrman et al., 1997; Relevy et al., 2015).

9-cis-RA and ATRA acted as inducers of ABCA1, ABCG1, and ApoE expression in J774 macrophages and THP-1

macrophages (Kiss et al., 2005) and RAW264.7

macrophages

(Schwartz et al. 2000), as well as inducers of cholesterol efflux to ApoA-1 in

RAW264.7

macrophages

(Langmann et al.,

2005).

Decreased cholesterol Clinical investigation accumulation through reported that dietary downregulation of SR- supplementation of A mRNA expression lycopene reduced and lipid synthesis in plasma LDL-C level human monocytederived macrophages (HMDMs) and THP-1 macrophages

(Napolitano et al., 2007). Increased cholesterol efflux possibly through HMG-CoA reductase/ RhoA/PPAR $\gamma / \mathrm{LXR} \alpha /$ ABCA1 and caveolin pathway (Palozza et al., 2011).
An in vivo study showed that male Wistar rats fed with capsanthin exhibited an increase in plasma HDL-C, an upregulation of ApoA-5 and LCAT mRNA expression (Aizawa and Inakuma, 2009).

$\beta$-Carotene comprises several isomers (alltrans- $\beta \mathrm{c}$ and $9-$ cis- $\beta \mathrm{c}$ ), which increased plasma HDL-C and attenuated atherosclerosis in $\mathrm{LDLR}^{-/-}$mice (Harari et al., 2008), $\mathrm{ApoE}^{-/-}$ mice (Harari et al., 2013), and even in fibrate-treated patients (Shaish et al., 2006), which is related with transcriptional induction of ABCA1, ABCG1, and ApoE

(Bechor et al., 2016).

9-cis-RA inhibited foam cell formation and atherosclerosis by activation of $\mathrm{LXR} \alpha$ and upregulation of $\mathrm{ABCA} 1$ and ABCG1 expression in $\mathrm{ApoE}^{-/-}$mice fed with HFD (Zhou et al., 2015).

(Fuhrman et al., 1997; Sesso et al., 2005;

Palozza et al., 2012). Lycopene displays potent hypolipidemic PCSK9 and HMG-CoA reductase, thus increasing hepatic LDLR (Sultan Alvi et al., 2017). effects via inhibiting 
TABLE 1-Continued

\begin{tabular}{|c|c|c|c|}
\hline Chemical class & Natural Compounds & In Vitro Studies & $\begin{array}{l}\text { Animal Studies, Clinical } \\
\text { Studies, and Targets }\end{array}$ \\
\hline & Ursolic acid & $\begin{array}{l}\text { Promoted ApoA-1- } \\
\text { mediated cholesterol } \\
\text { efflux from LDL- } \\
\text { loaded macrophages } \\
\text { through autophagy } \\
\text { (Leng et al., 2016). }\end{array}$ & $\begin{array}{l}\text { Reduced atherosclerotic } \\
\text { lesion size, along with } \\
\text { an increase of } \\
\text { macrophage autophagy } \\
\text { in LDLR }{ }^{-1-} \text { mice (Leng } \\
\text { et al., 2016). Ursolic } \\
\text { acid is } \\
\text { a pharmacological } \\
\text { inhibitor of ACAT1 and } \\
\text { ACAT2 (Lee et al., } \\
\text { 2006). }\end{array}$ \\
\hline & Betulinic acid & $\begin{array}{l}\text { Induced cholesterol } \\
\text { efflux through } \\
\text { blocking NF- } \kappa \text { B/ } \\
\text { miRNA-33s/ABCA1 } \\
\text { signaling pathway in } \\
\text { LPS-treated } \\
\text { macrophages (Zhao } \\
\text { et al., 2013a) and } \\
\text { increased ABCA1/ } \\
\text { ABCG1-mediated } \\
\text { cholesterol efflux in } \\
\text { both RAW264.7 and } \\
\text { THP-1 cells (Zhao } \\
\text { et al., 2013a). } \\
\text { Increased ApoA-1- } \\
\text { mediated cholesterol } \\
\text { efflux by inhibiting } \\
\text { ABCA1 degradation } \\
\text { in THP-1 } \\
\text { macrophages (Wang } \\
\text { et al., 2017f). }\end{array}$ & $\begin{array}{l}\text { Increased ABCA1 } \\
\text { expression and } \\
\text { enhanced fecal } \\
\text { cholesterol excretion, } \\
\text { along with suppressed } \\
\text { macrophage positive } \\
\text { areas in the aorta of } \\
\text { ApoE }{ }^{-/-} \text {mice (Gui } \\
\text { et al., 2016). Betulinic } \\
\text { acid is a potent } \\
\text { pharmacological } \\
\text { inhibitor of ACAT1 and } \\
\text { ACAT2 (Lee et al., } \\
\text { 2006). } \\
\quad \text { N.A. }\end{array}$ \\
\hline & poside Rb1 (Ginsen & $\begin{array}{l}\text { Ginsenoside Rb1 } \\
\text { increased ABCA1 } \\
\text { protein expression in } \\
\text { macrophage foam } \\
\text { cells (Liu et al., 2016c; } \\
\text { Qiao et al., 2017). } \\
\text { Ginsenoside Rd } \\
\text { inhibited SR-A } \\
\text { protein expression } \\
\text { and oxLDL uptake, } \\
\text { thus decreasing } \\
\text { intracellular } \\
\text { cholesterol content (Li } \\
\text { et al., 2011). }\end{array}$ & $\begin{array}{l}\text { Ginsenoside Rb1 } \\
\text { treatment reduced lipid } \\
\text { metabolism and } \\
\text { enhanced } \\
\text { atherosclerotic plaque } \\
\text { stability via enhancing } \\
\text { macrophage autophagy } \\
\text { and polarization (Liu } \\
\text { et al., 2016c; Qiao et al., } \\
\text { 2017). Ginsenoside Rd } \\
\text { treatment reduced the } \\
\text { oxLDL uptake and } \\
\text { atherosclerotic plaque } \\
\text { areas in ApoE }{ }^{-f-} \text { mice } \\
\text { (Li et al., 2011). }\end{array}$ \\
\hline & Saikosaponin A & $\begin{array}{l}\text { Suppressed } \\
\text { lipoprotein uptake by } \\
\text { diminishing LOX-1 } \\
\text { and CD36 expression, } \\
\text { as well as stimulated } \\
\text { cholesterol efflux } \\
\text { through upregulating } \\
\text { of ABCA1 and PPAR } \\
\text { expression (He et al., } \\
\text { 2016a). }\end{array}$ & N.A. \\
\hline
\end{tabular}


TABLE 1-Continued

Natural Compounds

Tanshinone IIA<smiles>Cc1coc2c1C(=O)C(=O)c1c-2ccc2c1CCCC2(C)C</smiles>

Tanshindiol C

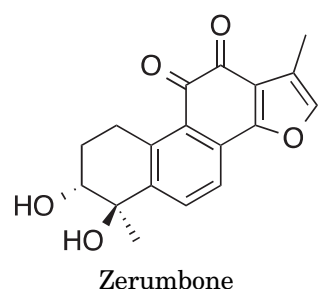<smiles>CC1=CCC(C)(C)C=CC(=O)C(C)=CC1</smiles>

Phenols

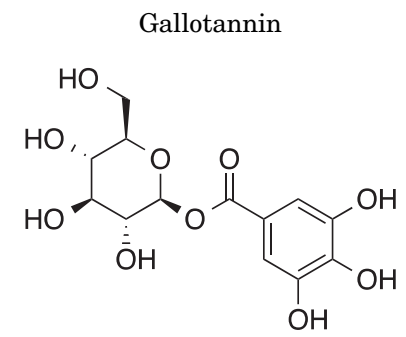

Curcumin<smiles>COc1cc(/C=C/C(=O)CC(=O)/C=C/c2ccc(O)c(OC)c2)ccc1O</smiles>

In Vitro Studies

Decreased oxLDL uptake, as well as CD36 expression in mouse macrophages (Tang et al., 2011), increased ABCA1/G1mediated cholestero efflux via the ERK/ Nrf2/HO-1 loop in THP-1-derived foam cells (Liu et al., 2014c).

Inhibited oxLDL induced foam cell formation via activation of Prdx1/ ABCA1 signaling pathway (Yang et al., 2018b).

Suppressed the SR-A and CD36 expression via regulating $\mathrm{AP}-1$ and $\mathrm{NK}-\kappa \mathrm{B}$

repression, leading to a blockade of acLDL uptake in THP-1

macrophages (Eguchi et al., 2007). Reduced cholesterol level via upregulation of ABCA1, coupled with the enhanced phosphorylation of ERK1/2 in THP-1 macrophages (Zhu and Liu, 2015)

Induced cholesterol efflux in oxLDLstimulated macrophages by increasing SR-BI/ ABCA1 expression (Zhao et al., 2015).

Ameliorated lipid accumulation in macrophages by both decreasing SR-Adependent oxLDL uptake via ubiquitin/ proteasome pathwayreduced SR-A expression, and increasing ABCA1dependent cholesterol efflux via LXR $\alpha$ induced ABCA1 protein expression (Kou et al., 2013; Min et al., 2013; Lin et al.,
Animal Studies, Clinical Studies, and Targets

Downregulated SR-A expression and ameliorated atherosclerotic lesions in aortas of $\mathrm{ApoE}^{-1}$

mice (Liu et al., 2014c).

Tanshinone IIA

promoted ABCA1dependent cholesterol efflux (Liu et al., 2014c) and upregulated LDLR in hyperlipidemic rats (Jia et al., 2016). A clinical trial has shown that tanshinone IIA reduced hs-CRP in patients with coronary artery disease (Li et al., 2017b).

N.A.

Prevented the development of atherosclerotic lesions in the cholesterol-fed rabbit model by reducing lipid level and oxidative stress (Hemn et al., 2013, 2015).

N.A.

Protected against atherosclerosis in $\mathrm{ApoE}^{-1-}$ mice (Zhao et al., 2012), and LDLR $^{-1-}$ mice (Hasan et al., 2014). Curcumin lowers LDL-C, and TG in patients at risk for CVD (Qin et al., 2017). Curcumin functions as an inhibitor of PCSK9 and upregulates hepatic LDLR expression (Tai et al., 2014). 
TABLE 1-Continued

Danshensu<smiles>O=C(O)C[C@H](O)Cc1ccc(O)c(O)c1</smiles>

6-Dihydroparadol<smiles>CCCCCCCC(O)CCc1ccc(O)c(OC)c1</smiles>

Paeonol<smiles>COc1ccc(C(C)=O)c(O)c1</smiles>

Polydatin<smiles>OC[C@H]1C[C@@H](O)[C@@H](O)C[C@@H]1Oc1cc(O)cc(/C=C/c2ccc(O)cc2)c1</smiles>

Protocatechuic acid<smiles>O=C(O)c1ccc(O)c(O)c1</smiles>

2015c; Soltani et al., 2017). Activation of AMPK/SIRT1/LXR $\alpha$ pathway (Lin et al., 2015c) and Nrf2/HO-1 pathway (Kou et al., 2013), and as well as inhibition of $\mathrm{p} 38$ MAPK pathway (Min et al., 2013) may be involved in its effects. Prevented cholesterol accumulation in mouse macrophages by inhibiting CD36mediated lipid uptake, while enhancing ABCA1/

G1-mediated cholesterol efflux (Wang et al., 2010b; Gao et al., 2016).

Promoted cholesterol efflux from THP-1 macrophages by increasing the expression of $\mathrm{ABCA} 1$ and $A B C G 1$ via preventing the proteasomedependent protein degradation (Wang et al., 2018a).

Promoted cholesterol efflux via activating LXR $\alpha /$ ABCA1

pathway in macrophages (Zhao et al., 2013b; $\mathrm{Li}$ et al., 2015c), inhibited cholesterol uptake through CD36 inhibition (Li et al., 2015c).

Activation of PPAR $\gamma$ dependent ABCA1 upregulation and decrease of CD36 expression (Wu et al., 2015b).

Promoted cholesterol efflux from macrophages by increasing $\mathrm{ABCA} 1$ and ABCG1 expression via reduction of miRNA10b expression (Wang et al., 2012a).
Attenuated high methionine-rich dietinduced accumulation of foam cells in rat aortic endothelium by attenuating TNF- $\alpha$ and ICAM1 expression

(Yang et al., 2010). Danshensu improved dyslipidemia by decreasing LDL-C and fatty acid by inhibiting HMG-CoA reductase and fatty acid synthase expression (Yang et al., 2011).

N.A.

Reduced atherosclerotic lesion formation and attenuated systemic inflammation as well as increased $\mathrm{ABCA} 1$

expression in $\mathrm{ApoE}^{-/-}$ mice (Zhao et al., 2013b). Paeonol reduced the levels of malondialdehyde and oxidzed LDL in hyperlipidemia rats (Dai et al., 2000).

Reduced TC, FC, CE, together with reduction of secretion of TNF- $\alpha$ and IL- $1 \beta$ in oxLDLstimulated $\mathrm{ApoE}^{-/-}$ mouse macrophages (Wu et al., 2015b). Polydatin improved dyslipidemia via suppressing PCSK9 and upregulation of hepatic LDLR expression (Li et al., 2018a).

Reduced the development of atherosclerosis in $\mathrm{ApoE}^{-1-}$ mice (Wang et al., 2010a, 2011; Stumpf et al., 2013). Protocatechuic acid could possibly regulate lipid metabolism via 
TABLE 1-Continued

In Vitro Studies

Salicylic acid<smiles>O=C(O)c1ccccc1O</smiles>

Salvianolic acid B<smiles>O=C(/C=C/c1ccc(O)c2c1[C@@H](C(=O)O[C@H](CO)Cc1ccc(O)c(O)c1)[C@@H](c1ccc(O)c(O)c1)O2)O[C@@H](CO)Cc1ccc(O)c(O)c1</smiles>

Sesamol<smiles>Oc1ccc2c(c1)OCO2</smiles>

Resveratrol<smiles>Oc1ccc(/C=C/c2cc(O)cc(O)c2)cc1</smiles>

Upregulated the expression of ABCA1 and SR-BI by AMPK activation or $\operatorname{PPAR} \alpha$ pathway, thereby stimulating cholesterol efflux from macrophages (Viñals et al., 2005; Lu et al., 2010).

Acted as an effective CD36 antagonist that blocks oxLDL uptake in mouse macrophages (Wang et al., 2010b) and

THP-1 macrophage (Bao et al., 2012), promoted cholesterol efflux via a PPAR $\gamma$ / LXR $\alpha /$ ABCA1-dependent pathway in THP 1 macrophages (Bao et al., 2012).

Decreased the expression of CD36, CD68, SR-A, and LOX-1 (Narasimhulu et al., 2018), and increased the expression/activity of PPAR $\gamma$ and LXR $\alpha$ in macrophages via a MAPK-dependent mechanism (Wu et al., 2015d).

Reduced oxLDL uptake (Voloshyna et al., 2013), promoted ApoA-1- and HDL mediated cholesterol efflux in both mouse and human

macrophages by increasing the expression of $\mathrm{ABCA} 1$ and ABCG1 via PPARv/LXR

(Berrougui et al., 2009; Allen and Graham, 2012) and adenosine $2 \mathrm{~A}$ receptor pathway (Voloshyna et al., 2013).
Animal Studies, Clinical Studies, and Targets

suppressing the expression of HMG-

CoA reductase (Liu et al., 2010b).

Aspirin attenuated atherosclerosis in ApoE $^{-1-}$ mice by suppressing systemic inflammation and promoting

inflammation resolution (Petri et al. 2017).

Exhibited

antiatherosclerotic effects in neointimal hyperplasia in rabbits (Yang et al., 2011) and in $\mathrm{ApoE}^{-1-}$ mice (Chen et al., 2006; Lin et al., 2007). Salvianolic acid $\mathrm{B}$ ameliorated hyperlipidemia via AMPK activation (Cho

et al., 2008) and inhibition of LDL oxidation (Yang et al., 2011).

Sesamol derivative (INV-403) and sesame oil prevent or regress atherosclerosis in

$\mathrm{LDLR}^{-/-}$mice (Narasimhulu et al., 2018) and

hyperlipidemic rabbits fed with an atherogenic diet by suppressing NF-

, $\kappa \mathrm{B}$ dependent vascular inflammation (Ying et al., 2011).

Exhibited antiatherosclerotic effects in several animal models, including $\mathrm{ApoE}^{-/-}$mice (Do et al., 2008; Chang et al., 2015), APOE*3Leiden.CETP mice (Berbée et al., 2013), and $\mathrm{ApoE}^{-/-} / \mathrm{LDLR}^{-/-}$ mice (Fukao et al., 2004). Resveratrol lowered the level of TC and TG in patients with dyslipidemia

(Simental-Mendia and Guerrero-Romero, 2019) and regulated lipid metabolism via inhibiting cholesterolester-transport protein and HMG-CoA expression/level (Cho et al., 2008). 
TABLE 1-Continued

Chemical class Natural Compounds

Phenylpropanoids<smiles>COc1cc(C[C@H]2COC(=O)[C@@H]2Cc2ccc(OC)c(OC)c2)ccc1O</smiles><smiles>C/C=C(/C)C(=O)OC[C@H]1[C@H](Cc2ccc(OC)c(OC)c2)CO[C@H]1c1ccc(OC)c(OC)c1</smiles><smiles>c1cc2c(cc1[C@@H]1OC[C@@H]3[C@H](c4ccc5c(c4)OCO5)OC[C@H]13)OCO2</smiles>

Honokiol

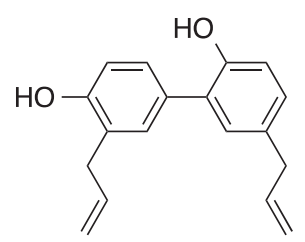

In Vitro Studies

Animal Studies, Clinical Studies, and Targets

Reduced cholesterol efflux from

macrophages by increasing ABCA1 expression via activating Nrf2dependent NF- $\kappa \mathrm{B}$ inhibitory effects (Jiang et al., 2012) and blocked oxLDLinduced upregulation of SR-A, thus reducing oxLDL uptake (Chen et al., 2017). Upregulated the expression of $\mathrm{ABCA} 1$, ABCG1, and ApoE, resulting in promotin cholesterol efflux in oxLDL-loaded THP-1 macrophages $(\mathrm{Xu}$ et al., 2013d) Increased cholesterol efflux from THP-1 macrophages by upregulating the expression of ABCA1 and ABCG1 (Wang et al., 2016a).

Inhibited oxLDLinduced cholesterol accumulation and enhanced cholesterol efflux from RAW264.7 macrophages via upregulation of PPAR $\gamma$, LXR $\alpha$, and ABCG1 (Liu et al., 2014a).

Activated the RXR/

LXR heterodimer in RAW264.7 cells, resulting in the induction of $\mathrm{ABCA} 1$ expression and enhancement of cholesterol efflux from MPMs (Kotani et al., 2010), and increased ABCG1 and ApoE expression in THP-1 macrophages (Jung et al., 2010). antiatherosclerotic and plaque-stabilizing effects in rats, rabbits, and $\mathrm{ApoE}^{-1-}$ mice (Chyu et al., 2004; Xu et al., 2014a; Wang et al., 2018e,f). EGCG prevented hyperlipidemia by increasing the expression and activity of LDLR (Lee et al., 2008).

Decreased cholesterol levels in mice (Huang et al., 2012a) and suppressed lipid accumulation and body weight gain in HFDinduced obese mice (Han et al., 2016). Reduced LDL-C level and postprandial serum glucose peaks due to the direct inhibition of 3-hydroxy-3-methylglutaryl-CoA reductase (HMGCR) and moderate PPAR $\gamma$ agonistic activity; however, no obvious effect on atherosclerotic plaque size was observed (Scharinger et al., 2016).

Prevented fat storage, decreased cholesterol level in serum (Lee et al., 2009c; Rogi et al., 2011). Attenuated atherosclerosis in $\mathrm{ApoE}^{-/-}$mice by suppressing vascular inflammation $(\mathrm{Wu}$ et al., 2010). The lipidlowering effect of sesamin was exerted through promoting the fecal excretion of sterols and inhibiting HMGCoA reductase (Liang et al., 2015). NA.
Displayed potential 
TABLE 1-Continued

Chemical class

Chlorogenic acid<smiles>O=C(/C=C/c1ccc(O)c(O)c1)O[C@@H]1C[C@@](O)(C(=O)O)C[C@H](O)[C@H]1O</smiles>

Caffeic acid<smiles>O=C(O)C=Cc1ccc(O)c(O)c1</smiles>

Ferulic acid<smiles>COc1cc(/C=C/C(=O)O)ccc1O</smiles>

Alkaloids<smiles>COC(=O)C1=CCCN(C)C1</smiles>

In Vitro Studies

Animal Studies, Clinical Studies, and Targets

Promoted macrophage Decreased level of cholesterol efflux serum cholesterol in through the PPAR $\gamma$ - hypercholesterolemic LXR $\alpha$-ABC rats by inhibition of transporters pathway HMG-CoA reductase (Park et al., 2015). (Rodriguez-Paez et al., 2003).

Enhanced HDLmediated cholesterol efflux from

Chlorogenic acid macrophages through increasing the expression of ABCG1 and SR-BI

(Uto-Kondo et al., 2010) and enhancement of $\operatorname{PPAR} \alpha$ ligand binding capacity in vitro ( $\mathrm{Kim}$ et al., 2014).

Decreased oxLDLelicited neutral lipid and cholesterol accumulation in RAW264.7

macrophages via increasing the transcription of PPAR $\gamma, \operatorname{LXR} \alpha$, ABCA1, and ABCG1 (Wu et al., 2014).

Increased the expression of ABCA1 and ABCG1 in macrophage form cells and further promoted cholesterol efflux (Chen and Wang, 2015). atherosclerosis in ApoE $\mathrm{E}^{-/-}$mice by inhibiting lipid accumulation and promoting cholesterol efflux via PPAR $\gamma /$ LXR $\alpha / \mathrm{ABCA} 1$ (G1) pathway (Wu et al., 2014).

Reduced the percentage and the total atherosclerotic lesion area as well as promoted

vasodilatation in cholesterol-rich diet-fed $\mathrm{ApoE}^{-1-}$ mice, and decreased levels of TC, LDL-C and TG in the serum (Wu et al., 2014). Caffeic acid reversed insulin resistance, dyslipidemia, hyperglycemia, inflammation, and oxidative stress in highfructose diet-induced metabolic syndrome in rats (Ibitoye and Ajiboye, 2018).

Ferulic acid suppressed atherosclerosis in ApoE $\mathrm{E}^{-/-}$mice by inhibiting the activities of hepatic ACAT and HMG-CoA reductase (Kwon et al., 2010). Ferulic acid also lowered TC, LDL-C, oxLDL, TG, and increased HDL-C in patients with dyslipidemia (Bumrungpert et al., 2018).

Promoted cholesterol Arecoline suppressed efflux by increasing ABCA1 expression (Ouyang et al., 2012). atherosclerosis in $\mathrm{ApoE}^{-1-}$ mice by

inhibiting $\mathrm{NF}-\kappa \mathrm{B}$ activation (Zhou et al., 2014).

Inhibited macrophage Suppressed foam cell formation by atherosclerosis promoting $\mathrm{LXR} \alpha /$ development in mice ABCA1-dependent (Feng et al., 2017; Shi cholesterol efflux (Lee et al., 2018; Zhu et al. et al., 2010). Prevented oxLDLinduced upregulation of LOX-1 and atherosclerosis in 2018). One report suggests that berberine promoted downregulation of SR- mice by enhancing 
Piperine<smiles>O=C(/C=C/C=C/c1ccc2c(c1)OCO2)N1CCCCC1</smiles>

Rutaecarpine<smiles>O=c1c2ccccc2nc2n1CCc1c-2[nH]c2ccccc12</smiles>

Evodiamine<smiles>CN1c2ccccc2C(=O)N2CCc3c([nH]c4ccccc34)[C@]21C</smiles><smiles>COc1cc(C(=O)OCCCCN=C(N)N)cc(OC)c1O</smiles>

Promoted ABCA1 protein expression in THP-1-differentiated macrophages by increasing ABCA1 protein stability by preventing calpainmediated ABCA1 protein degradation (Wang et al., 2017d). Upregulated expression of ABCA1 and SR-BI via $\mathrm{LXR} \alpha$ and $\operatorname{LXR} \beta$, thereby promoting cholesterol efflux (Xu et al., 2014b).

Promoted ApoA-1and HDL-mediated cholesterol efflux via the PPAR $\gamma / \mathrm{LXR} \alpha /$ ABCA1 and ABCG1 pathway (Jiang et al., 2017a). efflux from THP-1derived macrophages by directly binding to ABCA1 and thereby increasing ABCA1 stability (Wang et al., 2018c).
In Vitro Studies

BI in THP-1

macrophages (Guan et al., 2010; Chi et al. 2014a). It was reported to increase foam cell formation in human and mouse macrophages (Li et al., 2009b).

Animal Studies, Clinical Studies, and Targets

SR-A-mediated oxLDL uptake and foam cell

formation

(demonstrated in

human and mouse

nacrophages) through suppressing

phosphatase and tensin homolog expression, thus promoting the activation of Akt ( $\mathrm{Li}$ et al., 2009b). In patients with dyslipidemia, berberine reduced TC, LDL-C,

TG, and increased HDL-C, partially through inhibiting PCSK9 and increasing LDLR expression/ activity (Kong et al., 2004; Cameron et al., 2008; Ju et al., 2018).

Regulated lipid metabolism via increasing hepatic LDLR expression through proteolytic activation of SREBPs (Ochiai et al., 2015).

Reduced atherosclerotic plaque development, as well as macrophage and lipid content in

atherosclerotic plaques in $\mathrm{ApoE} \mathrm{E}^{-/-}$mice (Xu et al., 2014b). Lowered the level of TC, TG, and LDL-C, and hs-CRP in hyperlipidemic and hyperglycemic rats via AMPK activation and $\mathrm{NF}-\kappa \mathrm{B}$ inhibition (Nie et al., 2016; Tian et al., 2019b).

Increased cholesterol Decreased the size of atherosclerotic lesions and alleviated the hyperlipidemia, as well as hepatic macrovesicular steatosis in $\mathrm{ApoE}^{-/-}$ mice, probably via transient receptor potential vanilloid type 1 (TRPV1) pathway (Su et al., 2014).

Reduced atherosclerotic development in

$\mathrm{ApoE}^{-/-}$mice fed with atherogenic diet (Jiang et al., 2017a). 
TABLE 1-Continued

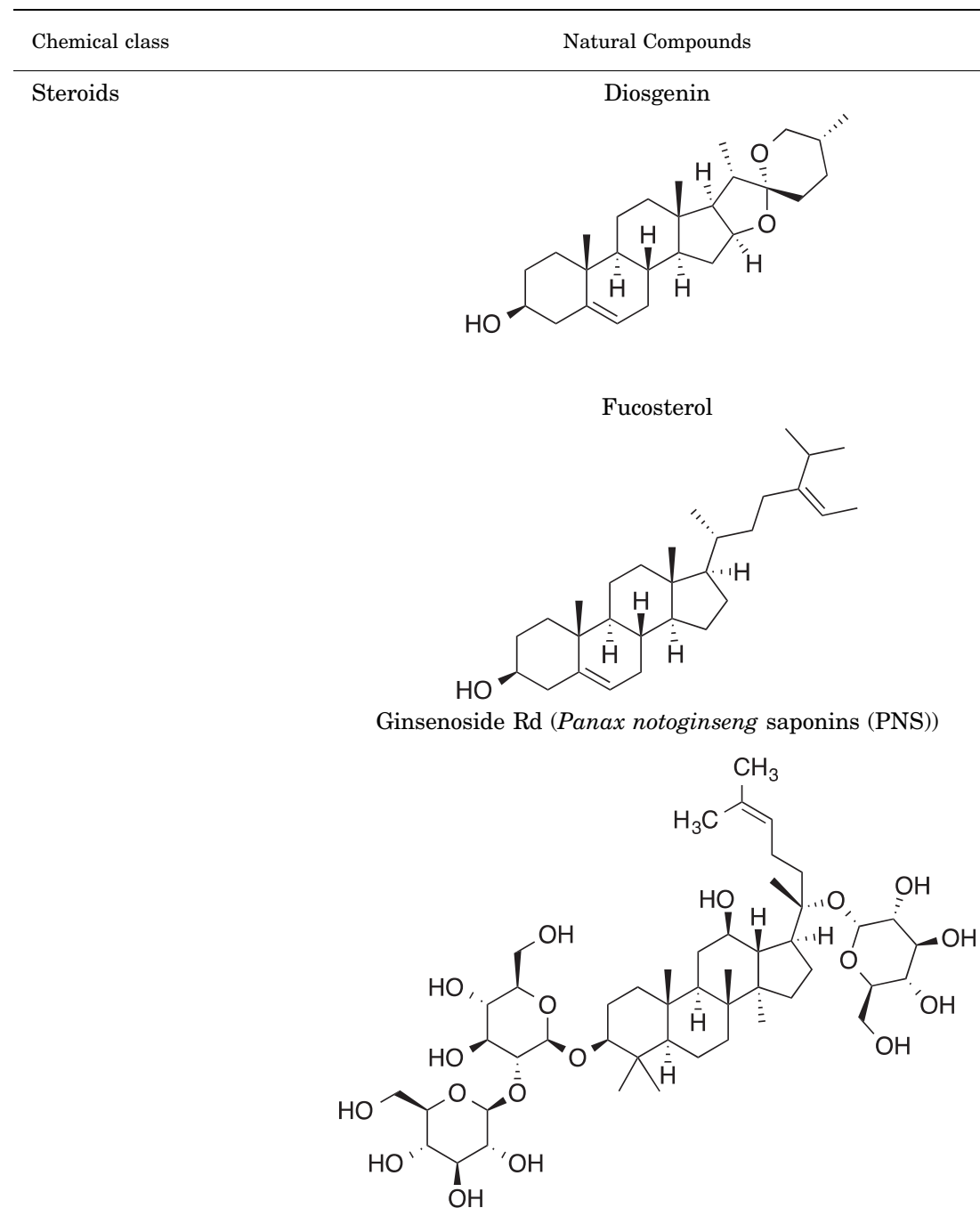

Vitamin $\mathrm{D}_{3}$ (Vitamin D)

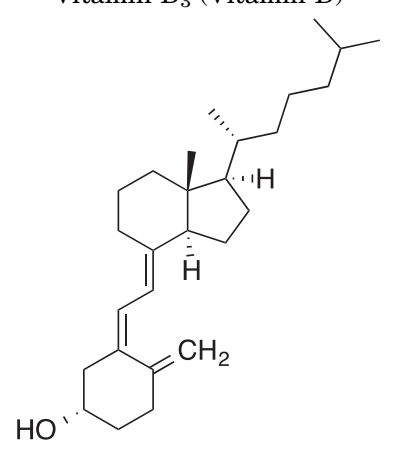

Fatty acids

Docosahexaenoic acid (DHA)

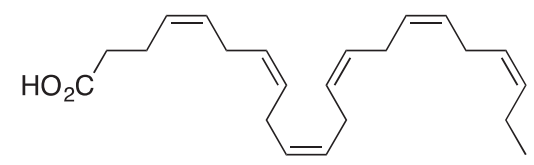

In Vitro Studies

Animal Studies, Clinical Studies, and Targets

Inhibited oxLDL

Inhibited

uptake by blocking

systemic

inflammation and

LOX-1/NF- $\kappa$ B

atherosclerosis in

$\mathrm{ApoE}^{-/-}$mice by

reducing $\mathrm{TC}$ and $\mathrm{CE}$ via promoting ABCA1-

pathway (Wang et al., dependent cholesterol

2017g). Promoted

cholesterol efflux by

increasing the $\mathrm{ABCA} 1$

expression

independent of $\operatorname{LXR} \alpha$

(Lv et al., 2015).

Promoted cholesterol efflux by increasing

the efflux

transporters ABCA1,

ABCG1, and ApoE

(Hoang et al., 2012).

Reduced LDL-C, and increased HDL-C (Hoang et al., 2012).
Decreased the accumulation of cholesterol esters via increasing ABCA1 expression (Jia et al., 2010).

Vitamin D inhibited CD36 and SR-Amediated lipid (oxLDL and ac-LDL) uptake (Oh et al., 2009; Yin et al., 2015)
Deficiency of vitamin D receptor (VDR) promoted modified LDL-induced foam cell formation of

macrophages from diabetic patients $(\mathrm{Oh}$ et al., 2015). Deficiency of macrophage VDR aggravated CD36 and SR-A-mediated lipid uptake (via JNK activation) to increase atherosclerosis in mice (Oh et al., 2015).

Vitamin D supplementation improved glycemic control, increased HDL$\mathrm{C}$ and decreased hsCRP levels in patients with CVD (Ostadmohammadi et al., 2019).

Inhibited the uptake of modified LDL in human macrophages partially through reduction of the expression of CD36 and SR-A (Pietsch et al., 1995), as well a
Reduced

atherosclerosis in

$\mathrm{ApoE}^{-1-}$ mice by reducing proinflammatory cytokine IL- $1 \beta$ (Alfaidi et al., 2018). Lowered TG in dyslipidemic 
TABLE 1-Continued

Chemical class

13-Hydroxyoctadecadienoic acid (13-HODE)

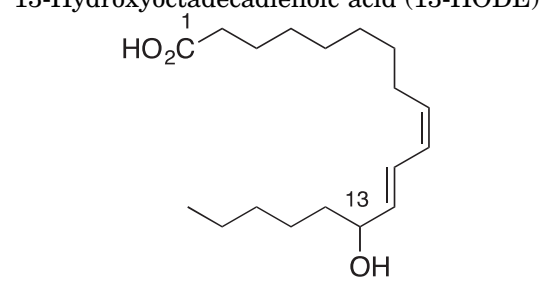

Linoleic acid

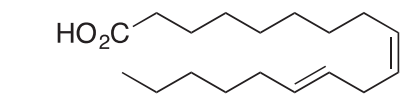

Amino acids

Carbohydrates<smiles>NC(=O)NCCC[C@H](N)C(=O)O</smiles>

$$
S \text {-allyl cysteine }
$$<smiles>C=CCSC[C@H](N)C(=O)O</smiles>

Polysaccharide isolated from Phellinus linteus N.A.

Astragalus polysaccharides

In Vitro Studies

of macropinocytosis and expression of syndecan-4 (McLaren et al., 2011b).

Inhibited the uptake of modified LDL in human macrophages partially through reduction of the expression of CD36 and SR-A (Pietsch et al., 1995), as well as of macropinocytosis and expression of syndecan-4 (McLaren et al., 2011b).

Promoted cholesterol efflux by activating PPAR/LXR $\alpha /$ ABCA1 and ABCG1 pathway (Kämmerer et al., 2011).

Functioned as an endogenous activator of $\operatorname{PPAR} \alpha, \operatorname{PPAR} \gamma$, and PGC1 $\alpha$, thus stimulating cholesterol efflux (Ringseis et al., 2008)

Promoted cholesterol efflux by increasing ABCA1 and ABCG1 expression in differentiated THP-1 macrophages (Tsuboi et al., 2018).

Increased ABCA1 expression, thus promoting cholesterol efflux in differentiated THP-1 macrophages (Malekpour-Dehkordi et al., 2013).

Promoted ApoA-1mediated cholesterol efflux by activating PPAR $\gamma / A B C A 1$ and ABCG1 pathway ( $\mathrm{Li}$ et al., 2015d).

Promoted ABCA1 expression in foam cells, thus increasing cholesterol efflux (Wang et al., 2010d).
Animal Studies, Clinical Studies, and Targets

patients (Weintraub, 2013).

Reduced and stabilized atherosclerotic plaques in $\mathrm{ApoE}^{-1-}$ and

$\mathrm{LDLR}^{-/-}$mice through its anti-inflammatory effects (Ringseis et al., 2006;

s Laguna-Fernandez et al., 2018). Lowered TG in dyslipidemic patients. EPA served as a substrate for resolvin E1 (RvE1), which promotes inflammation resolution i (Bäck and Hansson, 2019)

N.A.
Induced the regression of pre-established atherosclerotic plaques in $\mathrm{ApoE}^{-/-}$mice by promoting macrophage polarization toward a M2 antiinflammatory phenotype (McCarthy et al., 2013). Possibly reduced TC via increasing hepatic LDLR expression and activity (Ringseis et al., 2006).

Citrulline consumption promoted HDL- and ApoA-1-mediated cholesterol efflux by increasing the expression of both ABCA1 and ABCG1 (Uto-Kondo et al., 2014).

N.A

N.A.

N.A. 
TABLE 1-Continued

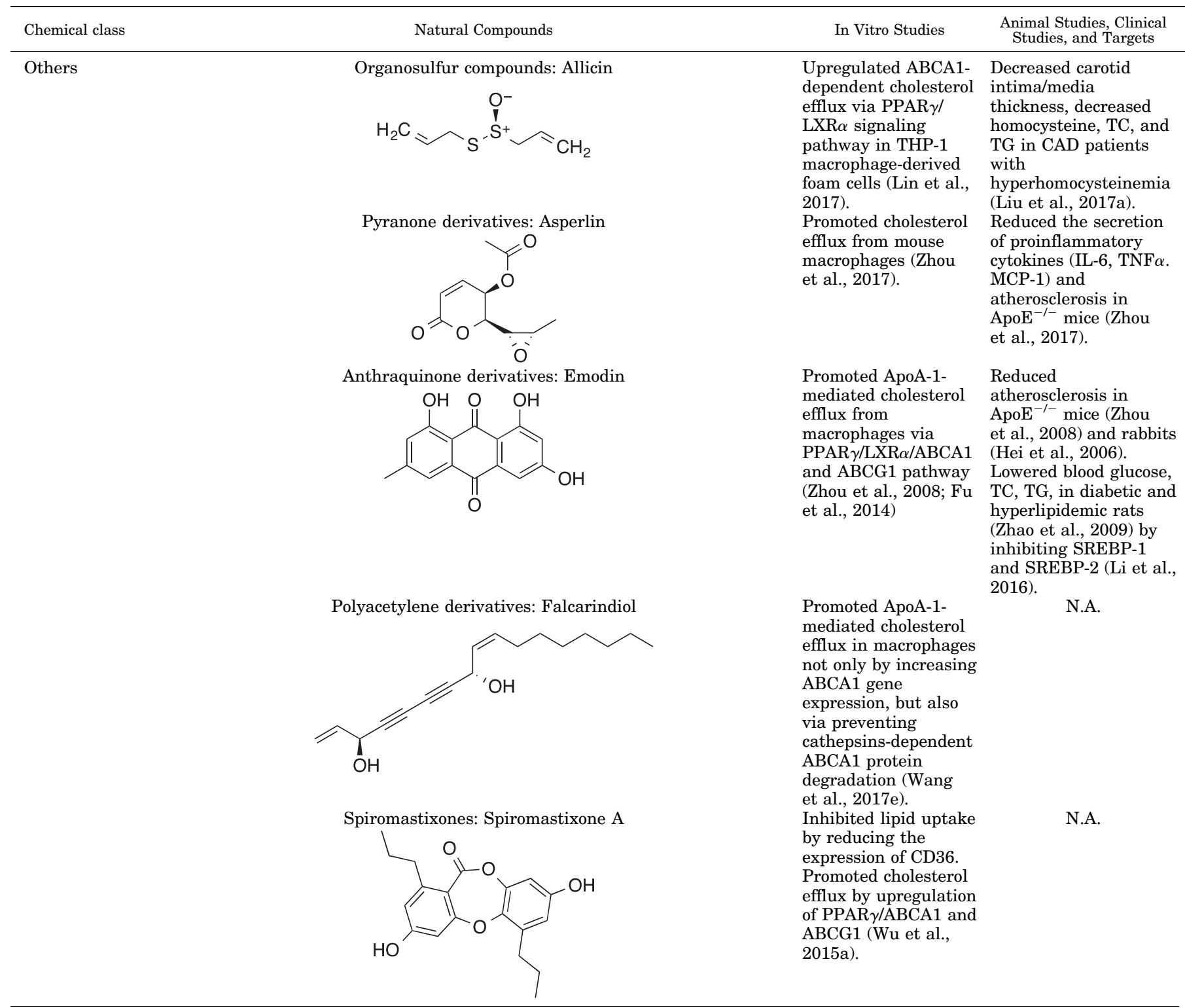

N.A., not available.

Further study indicated that the black rice AC-rich extract (300 $\mathrm{mg} / \mathrm{kg}$ body weight per day) inhibited the production of oxLDL and reduced the levels of TC and LDL-C, while upregulating the HDL-C level in serum from rats and $\mathrm{ApoE}^{-1-}$ mice, and also narrowed the area of atherosclerotic plaque and improved the stability of plaque to prevent the occurrence of embolism (Xia et al., 2006). Moreover, the ACs derived from pomegranate reduced blood lipids and exhibited an antiinflammatory activity (Wang et al., 2018b). It was reported that pomegranate peel extract containing ACs ( $1 \mathrm{~g} / \mathrm{kg}$ diet) has a slight effect on fatty streak formation and lipid metabolism in hypercholesterolemic rabbits (Sharifiyan et al., 2016). These studies indicated that the role of an AC-rich diet on the reduction of the risk of developing CVD might possibly be due to the ability of these compounds to inhibit lipid accumulation.

3. Baicalin. Baicalin, a glucuronide of baicalein, is a flavone glycoside that is present in the root of Scutellaria baicalensis Georgi with content as high as 12.1\% (Makino et al., 2008). The latter is a traditional Chinese medicine widely used to treat acute and chronic hepatitis, nephritis, and allergic diseases due to its antiinflammatory and hypolipidemic effects. One study indicated that baicalin $(50 \mu \mathrm{M})$ significantly increased HDL- but not ApoA1-mediated cholesterol efflux and upregulated the expression of SR-BI in a dose- and timedependent manner ( $\mathrm{Yu}$ et al., 2016). Another study showed that baicalin ( 50 and $100 \mu \mathrm{M}$ ) exerted potentially antiatherosclerotic effects by inhibition of form cell formation and lipid deposition via promoting cholesterol 
efflux through the PPAR $\gamma$-LXR $\alpha$-ABCA1/ABCG1 pathway in THP-1 macrophages (He et al., 2016b).

Baicalin also markedly decreased atherosclerotic lesion size and lipid accumulation in the carotid arteries in an atherosclerotic rabbit model (He et al., 2016b). Furthermore, long-term treatment with baicalin ( $80 \mathrm{mg} / \mathrm{kg}$ body weight) in rats with HFD markedly reduced the rising level of TC, LDL-C, NEFA, insulin, and TNF- $\alpha$ and also significantly ameliorated the fasting serum glucose levels (Guo et al., 2009), which suggests that baicalin may regulate the glucose/lipid metabolic disorders. Baicalin reduces TC, TG, LDL-C, and hs-CRP in patients with rheumatoid arthritis who have an increased risk of CAD (Hang et al., 2018).

4. Chrysin. Chrysin, also called 5,7-dihydroxyflavone, is a flavone present in blue passionflower (Passiflora caerulea), Pelargonium crispum, and Oroxylum indicum. It is one of the main active ingredients of propolis.

In a model of Wistar rats fed with HFD, chrysin (200 mg/kg body weight per day) decreased the mean levels of serum TC, TG, LDL-C, and VLDL-C (Anandhi et al., 2014). A recent report revealed that chrysin activated PPAR $\gamma$, the key regulator in lipid metabolism, to exert an anti-inflammatory effect (Feng et al., 2014). This suggested that chrysin might have an antiatherosclerotic effect, possibly by decelerating the formation of foam cells. Additional study displayed that HDLmediated RAW264.7 macrophages cholesterol efflux was significantly increased after chrysin $(10 \mu \mathrm{M})$ treatment by the upregulation of $\operatorname{PPAR} \gamma, \mathrm{LXR} \alpha, \mathrm{ABCA} 1$, and ABCG1 expression (Wang et al., 2015b). Additionally, chrysin also prevented the transcription of SR-A1 and SR-A2 but had no effect on CD36, suggesting that it may inhibit the cholesterol uptake by downregulating the expression of SR-A1 and SR-A2 (Wang et al., 2015b).

5. Cyanidin-3-O- $\beta$-glucoside. As a member of blueberry AC family, cyanidin-3-O- $\beta$-glucoside $(\mathrm{C} 3 \mathrm{G})$ is the most widely distributed $\mathrm{AC}$ in nature. C3G has antiinflammatory action, lowers the blood fat, as well as protects cardiovascular health through various mechanisms (Duchnowicz et al., 2012; Luo et al., 2012). It has been reported that $\mathrm{C} 3 \mathrm{G}(0.5,5$, and $50 \mu \mathrm{M})$ upregulated the expression of ABCG1 and ABCA1 in a dose-dependent manner and promoted the cholesterol efflux in human aortic endothelial cells (Wang et al., 2012c), suggesting a novel mechanism by which $\mathrm{C} 3 \mathrm{G}$ reduces oxidative damage on endothelial cells.

The antiatherosclerotic effect of C3G was recently evaluated in an HFD-induced atherosclerosis rat model. The results showed that diet supplementation with C3G $(150 \mathrm{mg} / \mathrm{kg})$ markedly decreased body weight, visceral adiposity, TG, TC, free fatty acids, and atherosclerosis index (Um et al., 2013). In $\mathrm{ApoE}^{-1-}$ mice, C3G (2 $\mathrm{g} / \mathrm{kg}$ diet) prevented the hypercholesterolemiainduced endothelial dysfunction and the development of atherosclerosis by inhibiting cholesterol and 7-oxysterol accumulation in the aorta (Wang et al., 2012b). Interestingly, it was shown that protocatechuic acid, a gut microbiota metabolite of $\mathrm{C} 3 \mathrm{G}$ in $\mathrm{ApoE}^{-/-}$mice, displayed a promising antiatherogenic effect at physiologically reachable concentrations $(0.25-1 \mu \mathrm{M})$, accelerating the cholesterol efflux in acLDL-loaded MPMs or THP-1 macrophages by interfering with the regulation of miRNA-10b-ABCA1/ABCG1 cascade (Wang et al., 2012a). Therefore, the gut metabolites of AC could also be explored as potential novel molecules for atherosclerotic prevention and treatment. Furthermore, a double-blind, randomized, placebo-controlled trial showed that consumption of anthocyanin increased HDL-C, decreased LDL-C, and promoted cellular cholesterol efflux in hyperlipidemia patients (Qin et al., 2009).

6. Daidzein. Daidzein is a naturally occurring isoflavone found in soybeans and other legumes. Because it is a nonsteroidal compound with estrogen-like biologic activities, daidzein is also regarded as a phytoestrogen. There are many clinical studies that investigated the effects of daidzein on the treatment of hypertension and menopausal syndrome, as well as alcoholism (Liu et al., $2014 d, e)$. Studies conducted over the last several decades showed that it has antiosteoporosis, antioxidative, hypolipidemic, and cardiovascular-protective effects (Robb and Stuart, 2014). Daidzein $(25 \mu \mathrm{M})$ induced paraoxonase-1 (PON-1) activity and protected LDL from oxidation in Huh7 cells, thereby displaying an antiatherosclerotic potential (Schrader et al., 2012). It was known that PON-1 may regulate cholesterol efflux by stimulating the $\operatorname{PPAR} \gamma-\mathrm{LXR} \alpha-\mathrm{ABCA} 1$ pathway (Ikhlef et al., 2016), indicating that daidzein may regulate macrophage cholesterol efflux. Additionally, in another study utilizing cultured HepG2 cells, daidzein $\left(\mathrm{EC}_{50}=3.21 \mu \mathrm{M}\right)$ upregulated the activity of CD36 and lysosomal integral membrane protein-II analogous1 (CLA-1), which is a homolog of SR-BI, suggesting that daidzein may affect lipid uptake (Yang et al., 2009). Daidzein was also reported to be an inhibitor of HMGCoA reductase, ACAT1, and ACAT2 (Borradaile et al., 2002; Sung et al., 2004).

In addition, in male middle-aged rats with HFD used to induce atherosclerosis, treatment with daidzein (30 mg/kg body weight) decreased the serum cholesterol and increased TG level (Sosić-Jurjević et al., 2007). In another study using rheumatoid arthritis rat model, it was shown that intragastric administration of daidzein $(20 \mathrm{mg} / \mathrm{kg}$ body weight) and hesperidin $(50 \mathrm{mg} / \mathrm{kg}$ body weight) reduced plasma VLDL, LDL-C, and TG concentrations, while increasing HDL-C levels (Ahmad et al., 2016).

7. Ellagic Acid. Ellagic acid, a dilactone of hexahydroxydiphenic acid, is an important antioxidant found in numerous fruits, such as cranberries, raspberries, grapes, pomegranates, as well as in walnuts. Ellagic acid has a wide range of biologic activities, including 
antiproliferative and antioxidative properties (Larrosa et al., 2010). One study utilizing J774A.1 murine macrophages suggested that ellagic acid ( 1 and $5 \mu \mathrm{M})$ stimulated cholesterol efflux by promoting the ABCA1 expression and upregulating PPAR $\gamma$ and LXR $\alpha$ levels (Park et al., 2011). This study also displayed that ellagic acid could modulate the SR-BI expression by counteracting PPAR $\gamma$-responsive early signaling. Additionally, it was demonstrated that pomegranate ellagic acid (PEA) $(10,20$, and $40 \mu \mathrm{g} / \mathrm{ml})$ regulated the expression of PPAR $\gamma$, ABCA1, and cholesterol $7 \alpha$-hydroxylase (CYP7A1) in the hepatic cell line L-02, thus regulating cholesterol metabolism (Lv et al., 2016). Also, pomegranate peel polyphenols, especially PEA ( 25 and $50 \mu \mathrm{g} / \mathrm{ml}$ ), inhibited macrophage lipid accumulation by decreasing the expression of CD36 and promoted ApoA1-mediated macrophage cholesterol efflux by upregulating ABCA1 and LXR $\alpha$ (Zhao et al., 2016).

In a hamster model with HFD, a high-dose of PEA ( $177 \mathrm{mg} / \mathrm{kg}$ body weight) decreased TC and TG in a dosedependent manner and upregulated the expression of $\mathrm{LXR} \alpha, \operatorname{PPAR} \alpha, \operatorname{PPAR} \gamma$, and their downstream gene ABCA1 (Liu et al., 2015). Moreover, using ApoE ${ }^{-1-}$ mice, it was shown that treatment of pomegranate phenolic compounds (200 $\mu \mathrm{g} / \mathrm{mouse}$ per day) for 3 months, mainly including gallic acid and ellagic acid, reduced atherosclerotic lesions (Aviram et al., 2008).

8. Hesperetin. Hesperetin is an aglycone of hesperidin, a natural bioflavonoid that is present in Leguminosae, Dimorphicaceae, Labiatae, and Rutaceae plants. Hesperetin has many biologic and pharmacological activities, such as antioxidative, anti-inflammatory, and antiatherosclerotic effects (Yang et al., 2012; Ren et al., 2016). It was shown that hesperetin $(5,10$, and $15 \mu \mathrm{M}$ ) reduced THP-1-derived foam cell formation by enhancing the expression of ABCA1 through increasing the activities of ABCA1 promoter and LXR enhancer, thus upregulating the ApoA1-mediated cholesterol efflux (Iio et al., 2012). Hesperetin $(25 \mu \mathrm{M})$ may act as an antiatherogenic agent possibly by also inhibiting oxLDLtriggered ROS in human umbilical vein endothelial cells (HUVECs) (Choi et al., 2008), as well as the proliferation and migration of VSMCs (Wei et al., 2016). A recent study showed that hesperetin reduced plasma TC level, endothelial dysfunction, macrophage infiltration, and atherosclerotic lesion in $\mathrm{ApoE}^{-1-}$ mice (Sugasawa et al., 2019).

9. Icariin. Icariin (ICA) is present in the traditional Chinese herbal medicine Epimedium brevicornum Maxim. In recent years, ICA has been studied for its effect on CVD. It was shown that ICA possessed atheroprotective functions through various mechanisms, including counteracting endothelial dysfunction, suppressing the proliferation and migration of VSMCs, as well as inhibiting foam cell formation and inflammatory responses (Fang and Zhang, 2017). In models of normal rats (Hu et al., 2016b) or $\mathrm{ApoE}^{-/-}$mice with HFD (Xiao et al., 2017), ICA (10-60 mg/kg body weight per day) significantly decreased the concentrations of TC, TG, and LDL-C. It was demonstrated that the inhibitory effects of ICA $(4 \mu \mathrm{M})$ on cholesterol intake and foam cell formation were accompanied by a reduced expression of CD36 and an upregulated SR-BI expression through p38 MAPK pathway in THP-1 cells (Yang et al., 2015). Additionally, ICA (5 and $10 \mu \mathrm{M})$ lessened RAW264.7 macrophage infiltration at atherosclerosis lesion by blocking the CX3CR1-CX3CL1 interaction, which is highly related to monocyte adhesion and migration (Wang et al., 2016c).

10. Iris Isoflavones. Iris isoflavones (tectorigenin, irstectorigenins, and iristectorins) present in rhizomes of Iris germanica L. have many biologic activities, such as antioxidative, anti-inflammatory, and antiangiogenic effects (Jung et al., 2003). Some of these nature products could also regulate the metabolism of blood sugar and cholesterol (Lee et al., 2000a; Jung et al., 2002). It was reported that iristectorigenin B (5 and $10 \mu \mathrm{M})$ acted as a novel LXR modulator by regulating the transcriptional activity of LXR $\alpha / \beta$ in RAW264.7 cells. It also induced the activation of ABCA1 and ABCG1, thus increasing the macrophage cholesterol efflux (Jun et al., 2012).

11. Pratensein. Pratensein is an isoflavone, which is present in Trifolium pretense L. (red clover). It was reported that pratensein $(10 \mu \mathrm{M})$ upregulated ABCA1 protein expression to increase the HDL levels in HepG2 cells (Gao et al., 2008). In the same line, another study indicated that pratensein $\left(\mathrm{EC}_{50}=1.08 \mu \mathrm{M}\right)$ upregulated the CLA-1 expression, which is a human homolog of SR-BI, suggesting that it can play a potential role in the process of cholesterol efflux in vitro (Yang et al., 2007, 2009).

12. Puerarin. Puerarin is present in the roots of Pueraria (Radix puerariae). Due to positive action on dilating coronary artery, protecting ischemic myocardium, resisting myocardial ischemia and re-injury, and preventing atherosclerosis (Bao et al., 2015), puerarin has been extensively studied for the treatment of CVD. It is demonstrated that puerarin $(25,50$, and $100 \mu \mathrm{g} / \mathrm{ml})$ decreased the cellular lipid accumulation in THP-1 macrophages by promoting ABCA1-mediated cholesterol efflux through pathways involving miRNA-7, serine/threonine kinase 11 (STK11), and the AMPactivated protein kinase (AMPK)-PPAR $\gamma$-LXR $\alpha$-ABCA1 cascade ( $\mathrm{Li}$ et al., 2017a). In the same model, puerarin (50 and $100 \mu \mathrm{g} / \mathrm{ml}$ ) suppressed lipid deposition and foam cell formation by downregulating the expression of CD36 (She et al., 2014). It (10, 50, and $100 \mu \mathrm{g} / \mathrm{ml})$ also suppressed oxLDL-induced macrophage activation and release of TNF- $\alpha$ and IL1- $\beta$ by inhibiting the TLR4/NF$\kappa \mathrm{B}$ pathway. Furthermore, treatment with puerarin (140 and $200 \mathrm{mg} / \mathrm{kg}$ body weight per day) decreased the level of blood glucose, TC, TG, LDL-C, and increased the HDL$\mathrm{C}$ level in the streptozotocin-induced diabetic rat model (Smith et al., 2006). 
13. Quercetin. Quercetin, a polyhydroxy flavonoid, is one of the most abundant natural polyphenols in different foods, such as onions, apples, broccoli, and ginkgo. It has broad biologic activities, including antiinflammatory, immunomodulatory, and cardiovascularprotective effects (Rauf et al., 2018). Some studies indicated that quercetin could interfere with foam cell formation (Lara-Guzman et al., 2012). Quercetin (50, 100, and $200 \mu \mathrm{M}$ ) enhanced ApoA1-mediated cholesterol efflux, as well as induced ABCA1 expression and the expression of PPAR $\gamma$ through activating the PPAR $\gamma$ signaling in THP-1-derived foam cells (Sun et al., 2015). Another study indicated that quercetinincreased ABCA1 expression possibly due to the p38dependent pathway (Chang et al., 2012). It was also shown that quercetin-driven ABCA1 upregulation could be mediated by enhancing $\mathrm{LXR} \alpha$ activity (Lee et al., 2013). Interestingly, a quercetin metabolite, quercetin3 -glucuronide, inhibited the formation of foam cells at $20 \mu \mathrm{M}$ by suppressing the expression of SR-A1 and CD36 in RAW264.7 cells (Kawai et al., 2008). Moreover, quercetin was reported to inhibit atherosclerosis by promoting ABCA1- and ABCG1-dependent RCT in $\mathrm{ApoE}^{-/-}$ mice (Cui et al., 2017).

14. Silymarin. Silymarin, a mixture of flavonolignans from the medicinal plant Silybum marianum, includes silybin (synonymous with silibinin), isosilybin, silydianin, silychristin, and little amounts of other phenolic compounds. Within the last decade, several studies have suggested that, in addition to its use in the treatment of liver diseases, silymarin also has a protective effect on CVD. It was shown that four compounds from silymarin (isosilybin A, silybin B, silychristin, and isosilychristin) induced the expression of ABCA1 protein in THP-1 cells. Especially isosilybin A (10 and $30 \mu \mathrm{M})$ promoted cholesterol efflux from THP-1 macrophages due to its PPAR $\gamma$-activating properties (Wang et al., 2015a). It is also reported that silymarin not only reduced the LDL and cholesterol levels, but also protected endothelial cells (Skottova and Krecman, 1998).

Furthermore, in the hypercholesterolemic rat model, supplementation of silybin (300 and $600 \mathrm{mg} / \mathrm{kg}$ body weight per day) in food significantly decreased serum levels of TC, TG, VLDL-C, LDL-C, and increased HDL-C, as well as reduced the formation of atherosclerotic plaques (Gobalakrishnan et al., 2016).

15. Wogonin. Wogonin, an $O$-methylated flavone, is present in the roots of Scutellaria baicalensis Georgi and the rhizomes of Scutellaria barbata L. It was shown that wogonin is effective in the treatment of inflammation, cancer, and anxiety (Tai et al., 2005; Li-Weber, 2009). Wogonin $(40 \mu \mathrm{M})$ attenuated oxLDL-induced cholesterol accumulation in murine J774.A1 macrophages by enhancing cholesterol efflux through increasing the ABCA1 protein expression (Chen et al., 2011). In addition, this flavonoid decreased phosphorylated levels of ABCA1 protein. Another beneficial effect of wogonin in the context of prevention and therapy of atherosclerosis is to protect physiologic function in vascular endothelial cells and VSMCs (Oche et al., 2016). An in vivo study suggests that wogonin ameliorated hyperglycemia and dyslipidemia in $\mathrm{db} / \mathrm{db}$ mice via PPAR $\alpha$ activation (Bak et al., 2014).

\section{B. Terpenoids}

Terpenoids, also known as isoprenoids, constitute one of the largest families of natural products, which, in contrast to terpenes, contain additional functional groups, mostly oxygen containing. Several bioactive terpenoids have been shown to be promising for therapeutic purposes (Goto et al., 2010). In this section, we focus on the inhibitory effects of terpenoids on foam cell formation and the associated molecular mechanisms of action.

1. Carotenoids. Carotenoids, also called tetraterpenoids, are a complex group of organic pigments with a basic tetraterpene skeleton and a variety of biochemical functions. A number of carotenoids have been reported to be associated with a wide range of bioactivities and potential health benefits (Rao and Rao, 2007; Fiedor and Burda, 2014). Epidemiologic studies, such as research regarding the association between $\beta$-carotene and CVD and case-control trails testing the concentration of $\beta$-carotene of patients with different CVD events, have demonstrated that the consumption of carotenoids can reduce the risk of CVD (Kohlmeier and Hastings, 1995; Riccioni et al., 2012). Here, the published studies regarding the effect of several main carotenoids, i.e., astaxanthin, $\beta$-carotene, capsanthin, retinoids, and lycopene, on cholesterol efflux and foam cells formation are discussed.

a. Astaxanthin. Astaxanthin is a major naturally occurring keto-carotenoid, mainly found in various microorganisms and marine animals and responsible for their pink-red pigmentation. Recently, there is a rapidly growing interest for its application in counteracting foam cell formation and stabilization and/or regression of atherosclerosis. It was shown that THP-1 macrophages incubated in the presence of astaxanthin (5-10 $\mu \mathrm{M})$ downregulated the SR-A and CD36 mRNA expression (48\% and $58 \%$, respectively), which is relevant to cholesterol uptake (Kishimoto et al., 2010). Further studies showed that astaxanthin promotes the ABCA1/G1 expression (up to 2.0- and 3.2-fold in protein level), resulting in increasing the ApoA-1/HDLmediated cholesterol efflux (116\% and $25 \%$, respectively) from RAW264.7 cells via an LXR-independent manner, but only at high concentrations (50 and $100 \mu \mathrm{M})$ (Ramírez-Tortosa et al., 1999; Iizuka et al., 2012). In agreement with the in vitro observation, a randomized, placebo-controlled clinical study was conducted in humans (aged 25-60 years) with mild hyperlipidemia (levels of $120-200 \mathrm{mg} / \mathrm{dl}$ ) for 12 -week 
astaxanthin administration (at doses of $0,6,12$, and $18 \mathrm{mg} /$ day). The results showed that 6 and $12 \mathrm{mg} /$ day doses of astaxanthin significantly increased HDL-C in correlation with increased serum adiponectin (Yoshida et al., 2010).

b. Capsanthin. Capsanthin is the main carotenoid in paprika and it does not possess provitamin A activity. There is some scientific evidence supporting the view that capsanthin has the potential to increase cholesterol efflux. An in vivo study showed that male Wistar rats fed with capsanthin (the low dose: $0.16 \mathrm{~g} / \mathrm{kg}$, the high dose: $0.32 \mathrm{~g} / \mathrm{kg}$ ) for 2 weeks exhibited an increase in plasma HDL-C and an upregulation of ApoA-5 and LCAT mRNA expression, without significantly influencing mRNA levels of other genes related to cholesterol metabolism (such as ABCA1, ApoA-1, and SR-BI) (Aizawa and Inakuma, 2009). In the same line, it was shown that supplementation of paprika significantly decreased the TC, HDL-C, atherogenic index values in rats fed with a high-cholesterol diet (Park et al., 2010).

c. $\beta$-Carotene. $\beta$-Carotene is a carotenoid with a hydrocarbon skeleton and high pro-vitamin A activity (Olson, 1989). Several studies provide insights into the effects of $\beta$-carotene on the cholesterol metabolism (Fuhrman et al., 1997; Relevy et al., 2015). An in vitro study indicated that treatment with $\beta$-carotene $(0-10 \mu \mathrm{M})$ results in a dose-dependent suppression of cholesterol synthesis by inhibiting the cellular HMGCoA reductase activity in $\mathrm{J} 774$ macrophages. On the other hand, a study demonstrated that $\beta$-carotene $(5 \mu \mathrm{M})$ could not significantly upregulate the ABCA1, ABCG1, and ApoE mRNA levels in human monocytes compared with retinoic acid (Langmann et al., 2005).

Natural $\beta$-carotene comprises several isomers, including all-trans- $\beta$-carotene (all-trans- $\beta \mathrm{c}$ ) and 9-cis$\beta$-carotene (9-cis- $\beta \mathrm{c})$. There is a lot of evidence from cellular, animal, and clinical studies supporting the association between the $\beta$-carotene isomers and the cholesterol levels. For example, a recent study conducted in RAW264.7 macrophages revealed that alltrans- $\beta$ c and 9 -cis- $\beta$ c-regulated cholesterol efflux to HDL was related to transcriptional induction of ABCA1, ABCG1, and ApoE (Bechor et al., 2016). In addition, previous study showed that the alga Dunaliella bardawil (containing high levels of all-trans- $\beta \mathrm{c}$ and 9 -cis- $\beta \mathrm{c}$ ) increased plasma HDL-C and attenuated atherosclerosis in both $\mathrm{ApoE}^{-/-}$(Harari et al., 2013) and $\mathrm{LDLR}^{-/-}$mice (Harari et al., 2008). A clinical study indicated that 9 -cis- $\beta$ c-rich powder of the alga Dunaliella bardawil increased plasm HDL-C in fibratetreated patients (Shaish et al., 2006). It was also reported that 9 -cis- $\beta \mathrm{c}$ can be cleaved by endogenous $\beta$-carotene $15,15^{\prime}$-monooxygenase 1 to form 9-cis retinoic acid or other retinoids, subsequently activating the RXR and finally inhibiting foam cell formation and atherosclerosis progress (Zolberg Relevy et al., 2015). d. Retinoids. Plentiful evidence suggests that retinol (also known as vitamin $\mathrm{A}_{1}$ ) and its natural derivatives 9-cis retinoic acid (9-cis-RA) and all-trans retinoic acid (ATRA) can increase macrophage cholesterol efflux and, therefore, are promising therapeutic agents for the prevention of atherosclerosis progression. It was reported that 9-cis-RA and ATRA $(5 \mu \mathrm{M})$ are potent inducers of ABCA1, ABCG1, and ApoE expression in THP-1 macrophages, as well as exhibited a very strong induction of cholesterol efflux to ApoA-1 in RAW264.7 macrophages (Langmann et al., 2005). Another study revealed that retinoids are able to induce the CYP27 expression, which is likely to act as a modulator of the LXR activation, subsequently mediating cholesterol efflux from macrophages (Szanto et al., 2004). This notion is further underscored by the observation that the cholesterol efflux induced by retinoids involves the activation of steroidogenic acute regulatory protein expression and the upregulation of LXR-targeted genes (SREBP-1c and ABCA1) in mouse macrophages (Manna et al., 2015).

Furthermore, 9-cis-RA has been reported to increase ABCA1-mediated cholesterol efflux from J774 macrophages, THP-1-derived macrophages (Kiss et al., 2005), and RAW264.7 macrophages (Schwartz et al., 2000). To further characterize the underlying molecular mechanisms of 9-cis-RA on cholesterol efflux, a study using J774A.1 cells and primary peritoneal macrophages showed that 9-cis-RA-mediated inhibition of foam cell formation and amelioration of atherosclerosis were regulated by the activation of $\operatorname{LXR} \alpha$ and the upregulation of ABCA1 and ABCG1 expression (Zhou et al., 2015). Interestingly, treatment with 9-cis-RA $(2 \mathrm{mg} / \mathrm{kg})$ can ameliorate the atherosclerosis in $\mathrm{ApoE}^{-/-}$ mice fed with HFD (Zhou et al., 2015).

Another study indicated that ATRA-induced upregulation of CD36 mRNA and protein expression were related to the retinoic acid receptor-dependent signaling in THP-1 cells, which may contribute to the foam cell formation and the progress of atherosclerosis (Wuttge et al., 2001). Further work also studied regulatory action of retinoids in this context; ATRA could increase the cholesterol efflux to ApoA- 1 and enhance the ABCA1 expression, both at the mRNA and protein level in MPMs and HMDMs, in a dose-dependent manner $(0.5-10 \mu \mathrm{M})$. Furthermore, ATRA treatment also increased the ABCG1 and SREBP-1c mRNA expression (Costet et al., 2003). Moreover, ATRA-increased levels of ABCA1 protein and mRNA may partly depend on the induction of LXR in THP-1 cells (Wågsäter et al., 2003).

e. Lycopene. Lycopene is the most abundant carotenoid pigment in tomato and contributes to the red color of tomatoes. It does not have pro-vitamin A activity but has many other biologic effects. Recently, this carotenoid has received marked attention for its potential in preventing CVD and interference with foam cell formation (Mozos et al., 2018). It was shown that lycopene 
$(10 \mu \mathrm{M})$ decreased cholesterol accumulation through downregulation of the SR-A mRNA expression and lipid synthesis, along with increase in the secretion of IL-10 in HMDMs and THP-1 macrophages (Napolitano et al., 2007). These data are in agreement with another study in THP-1 cells, which showed that lycopene $(10 \mu \mathrm{M})$ suppressed the cholesterol synthesis and efflux (Palozza et al., 2011). Furthermore, the potential role of lycopene $(0.5-2 \mu \mathrm{M})$ in attenuating foam cell formation through a cascade mechanism may involve HMG-CoA reductase inhibition, RhoA inactivation, subsequent increase in PPAR $\gamma$ and $\mathrm{LXR} \alpha$ activation, and enhancement of ABCA1 and caveolin 1 expression ultimately (Palozza et al., 2011). In this line, there is a known association between the PPAR $\gamma$ activation and cholesterol efflux from peritoneal macrophages in inflammation $(\mathrm{He}$ et al., 2014).

In agreement with these in vitro observations, the studies conducted on animal models (rabbits, rats, and mice) showed that lycopene reduced the serum LDL-C and increased the serum HDL-C levels (Palozza et al., 2012). Mechanistically, lycopene displays potent hypolipidemic effects via inhibiting PCSK9 and HMG-CoA reductase, thus increasing hepatic LDLR (Sultan Alvi et al., 2017). A human intervention trial also suggested that diet with dietary supplementation of lycopene $(60 \mathrm{mg} /$ day $)$ in healthy men resulted in a significant decrease $(14 \%)$ in the LDL-C concentration (Fuhrman et al., 1997). However, another clinical investigation reported that dietary supplementation of lycopene is not associated with a reduced risk of CVD in middleaged and older men (Sesso et al., 2005).

2. Ursolic Acid. Ursolic acid (UA), a natural ursanetype pentacyclic triterpenoid, is abundantly distributed in the plant kingdom. Recently, one study showed that UA $(10 \mu \mathrm{M})$ promoted cholesterol flux from LDL-loaded macrophages to ApoA-1 (not HDL) through autophagy, without altering mRNA or protein levels of ABCA1 and ABCG1 in MPMs (Leng et al., 2016). Furthermore, UA $\left(50 \mathrm{mg} / \mathrm{kg}\right.$ ) treatment in $\mathrm{LDLR}^{-/-}$mice significantly reduced atherosclerotic lesion size, along with increase of macrophage autophagy (Leng et al., 2016). Taken together, these results provided evidence that UA may have potential to be further studied as an antiatherogenic agent.

3. Betulinic Acid. Betulinic acid, which is derived from the bark of yellow and white birch trees, is a pentacyclic triterpenoid with a wide range of pharmacological properties. One study indicated that betulinic acid may induce cholesterol efflux through blocking the NF- $\kappa$ B-miRNA-33s-ABCA1 signal pathway in LPS-treated macrophages (Zhao et al., 2013a). Consistently, in both RAW264.7 and THP-1 cells, betulin (a betulinic acid derivative) significantly increased ABCA1/ABCG1-mediated cholesterol efflux via suppressing the expression of SREBPs, which bind to E-box motifs in the ABCA1 promoter (Gui et al., 2016).
Betulinic acid was reported to be a potent pharmacological inhibitor of ACAT1 and ACAT2 (Lee et al., 2006), suggesting its potential utility in reduced $\mathrm{CE}$ accumulation. Moreover, an in vivo study was done using $\mathrm{ApoE}^{--}$mice in which increased ABCA1 expression and enhanced fecal cholesterol excretion were observed upon long-term administration of betulin $(40 \mathrm{mg} / \mathrm{kg})$, along with suppressed macrophage-positive areas in the aortic sinuses (Gui et al., 2016). Another study confirmed that betulinic acid $(50 \mathrm{mg} / \mathrm{kg})$ reduced atherosclerotic lesions and reduced TG, TC, and LDL-C levels in $\mathrm{ApoE}^{-/-}$mice (Zhao et al., 2013a).

4. Erythrodiol. Erythrodiol is a pentacyclic triterpenoid present in olive oil. Recently, a study was conducted in THP-1 macrophages to screen the effect of various components of olive oil on foam cell formation. The results showed that among several compounds, only erythrodiol $(10 \mu \mathrm{M})$ has a positive effect on ApoA-1mediated cholesterol efflux by inhibiting ABCA1 degradation (Wang et al., 2017f). Therefore, erythrodiol may serve as a good candidate for further studies related to the prevention of atherosclerosis progression.

5. Ginsenosides. Ginseng, the original sources of which are the roots of the plant species Panax ginseng, is therapeutically used throughout the world for its possible curative and health-restorative properties. Most of the pharmacological actions of ginseng are attributed to its major constituents, ginsenosides. There are several types of ginsenosides, including Rb1, Rg1, Rg3, Rh1, Re, and Rd. Numerous studies focused on the potential benefits of the ginseng extract and its purified ginsenoside monomers in the area of CVD (Lee and Kim, 2014). Among these various ginsenosides, ginsenosides $\mathrm{Rb} 1$ and $\mathrm{Rg} 1$ are regarded as the most abundant active components from ginseng. A study showed that ginsenoside $\mathrm{Rb} 1(10 \mu \mathrm{M})$ significantly increased cholesterol efflux from foam cells to ApoA-1 by about 21\% (Wang et al., 2007b). Similarly, a recent publication indicated that $\mathrm{Rb1}(10-80 \mu \mathrm{M})$ treatment showed a significant increase in ABCA1 protein expression in macrophage foam cells, which provided the indirect evidence for a possible effect on the cholesterol efflux. Rb1 treatment also reduced the lipid metabolism and enhanced atherosclerotic plaque stability via enhancing macrophage autophagy in primary peritoneal macrophages isolated from C57BL/6 mice and $\mathrm{ApoE}^{-/-}$mice (Qiao et al., 2017).

Ginsenoside Rd is another important constituent of ginseng. Data obtained from a RAW264.7 cell model revealed that $\mathrm{Rd}(20 \mu \mathrm{M})$ inhibited SR-A protein expression, followed by the decrease of oxLDL uptake and decreased intracellular cholesterol content, which is probably mediated by the inhibition of $\mathrm{Ca}^{2+}$ influx through $\mathrm{Ca}^{2+}$ channels (Li et al., 2011). Consistently, an in vivo study indicated that $R d$ treatment $(20 \mathrm{mg} / \mathrm{kg}$ per day) reduced the oxLDL uptake and atherosclerotic plaque areas in $\mathrm{ApoE}^{-/-}$mice. 
6. Saikosaponin A. Saikosaponin A, a triterpenoid glycoside (saponin), is the major bioactive constituent from the Chinese herb Radix bupleuri. Saikosaponin A (at concentrations of $0-50 \mu \mathrm{M}$ ) ameliorated oxLDLinduced foam cell formation and suppressed lipoprotein uptake by diminishing LOX-1 and CD36 expression, as well as stimulated cholesterol efflux through upregulating the ABCA1 and PPAR $\gamma$ expression (He et al., 2016a). Saikosaponin A also regulated the immune inflammatory reaction via PI3K/Akt/NF- $\kappa \mathrm{B} / \mathrm{NLRP} 3$ signaling pathway and decreased the diffusion of proinflammatory cytokines (IL-1 $\beta$, IL-18, IL-6, TNF- $\alpha$, and MCP-1), revealing new insight on the potential of saikosaponin A in the context of atherosclerosis ( He et al., 2016a).

7. Tanshinone IIA. Danshen, the rhizome of Salvia miltiorrhiza Bunge, has been widely used to treat various diseases for centuries (Gao et al., 2012). The chemical constituents and biologic activities of Danshen have been extensively studied. Among its constituents, tanshinone IIA is the major bioactive lipophilic compound (Xu and Liu, 2013; Fang et al., 2018). An in vitro study demonstrated that tanshinone IIA (at concentrations of $0.1-10 \mu \mathrm{M}$ ) decreased cellular cholesterol level, oxLDL uptake, as well as CD36 expression at both the mRNA and protein levels in oxLDL-treated MPMs (Tang et al., 2011). Furthermore, tanshinone IIA (1-10 $\mu \mathrm{M})$ induced the reduction of CD36, which might be related to a PPAR $\gamma$ antagonism (Tang et al., 2011). Tanshinone IIA also increased the ABCA1- and ABCG1-mediated cholesterol efflux through the ERK/ Nrf2/HO-1 loop and decreased the SR-A-mediated oxLDL uptake by inhibition of AP-1, resulting in decreasing cholesterol accumulation in cells (Liu et al., 2014c).

In vivo, tanshinone IIA (at doses of $10-90 \mathrm{mg} / \mathrm{kg}$ ) also downregulated the SR-A mRNA expression and ameliorated the atherosclerotic lesions in the aortas of $\mathrm{ApoE}^{-/-}$mice (Tang et al., 2011). Consistently, another study also showed that tanshinone IIA $(30 \mathrm{mg} / \mathrm{kg}$ per day) lessened atherosclerotic plaques in $\mathrm{ApoE}^{-1-}$ mice (Liu et al., 2014c). Recently, another study conducted in macrophages from the peritoneal cavity of rats and THP-1-derived macrophages showed that ABCA1 mRNA and protein expression were significantly increased, while CD36 was significantly reduced upon tanshinone IIA treatment, thereby demonstrating simultaneous effects on cholesterol intake and efflux (Jia et al., 2016). In addition, tanshinone IIA was also reported to upregulate LDLR in hyperlipidemic rats (Jia et al., 2016). Furthermore, a clinical trial has shown that tanshinone IIA reduces hs-CRP in patients with CAD (Li et al., 2017b).

8. Tanshindiol $C$. Tanshindiol $\mathrm{C}$ is another bioactive compound isolated from Danshen. A recent report showed that tanshindiol $\mathrm{C}(1,3$, and $10 \mu \mathrm{M})$ concentration dependently inhibits oxLDL-induced foam cell formation via activation of Prdx1/ABCA1 signaling pathway. Also, tanshindiol $\mathrm{C}$ treatmentinduced Prdx1 transcription was coregulated by Nrf2 and Sirt1 expression (Yang et al., 2018b). Furthermore, tanshindiol $\mathrm{C}$ also significantly inhibited the secretion of TNF- $\alpha$, IL- $1 \beta$, and IL-8 (Yang et al., 2018b). These data indicated that the potential therapeutic effects of tanshindiol $\mathrm{C}$ on atherosclerosis could be due to the modulation of both foam cell formation and inflammation. However, more conclusive evidence is needed to confirm the significance of tanshindiol $\mathrm{C}$ in the context of atherosclerosis in animal models and clinical trials.

9. Zerumbone. Zingiber zerumbet is a wild ginger commonly found in Asia. It has been used as a traditional herbal medicine to treat various diseases for a long time. A major bioactive component isolated from Zingiber zerumbet is zerumbone, which is a natural cyclic sesquiterpene known to possess numerous biologic activities. Zerumbone was reported to have therapeutic potential in the context of atherosclerosis. In vitro experiments showed that zerumbone (5 and $10 \mu \mathrm{M})$ suppressed the SR-A and CD36 mRNA expression via regulating $\mathrm{AP}-1$ and $\mathrm{NF}-\kappa \mathrm{B}$ repression, leading to a blockade of acLDL uptake in THP-1 macrophages (Eguchi et al., 2007). Furthermore, THP-1 macrophages treated with zerumbone $(10-100 \mu \mathrm{M})$ showed a significant reduction in the cholesterol levels via upregulation of mRNA and protein levels of ABCA1, but not ABCG1, coupled with the enhanced phosphorylation of ERK1/2 (Zhu and Liu, 2015). The consensus regarding the beneficial effects of zerumbone is strongly supported by experiments conducting in a cholesterol-fed rabbit model, which displayed that zerumbone $(8-20 \mathrm{mg} / \mathrm{kg})$ could prevent the development of atherosclerotic lesions (Hemn et al., 2013, 2015).

\section{Phenolic Compounds}

1. Gallotannin. 1,2,3,4,6-Penta-O-galloyl- $\beta$-d-glucose (PGG) is a prodrug of gallotannin, which is present in diverse medicinal herbs. Zhao et al. (2015) found that PGG $(2.5$ and $5 \mu \mathrm{M})$ induced cholesterol efflux in oxLDL-stimulated J774A.1 and THP-1 macrophages by increasing SR-BI/ABCA1 expression, indicating that PGG has the potential to be further studied for promoting RCT and conferring atheroprotective effects.

2. Curcumin. Curcumin is a potent antioxidant present in Curcuma longa (turmeric or Jiang Huang) that has been demonstrated to confer protection against atherosclerosis in $\mathrm{ApoE}^{-/-}$(Zhao et al., 2012) and $\mathrm{LDLR}^{-/-}$mice (Hasan et al., 2014). It has been shown that curcumin and other bioactive phenolic compounds from turmeric have powerful bioactivities, including antioxidation associated with preventing lipid peroxidation (Ramirez Bosca et al., 1997). Curcumin was also used as a tool compound for inhibiting histone methyltransferase p300, c-Jun N-terminal kinases (JNK), and transcriptional factor AP-1 (Li et al., 2004b; Lee et al., 
2009b; Huwait et al., 2011). By modifying AP-1, curcumin inhibited the resistin-induced dysregulation of SR-A, CD36, and ABCA1 (Lee et al., 2009b). In 2012, Zhao et al. (2012) systematically analyzed the effects of curcumin on macrophage-derived foam cell formation. The authors observed that curcumin $(20$ and $40 \mu \mathrm{M})$ significantly ameliorated lipid accumulation in J774.A1 macrophages induced by oxLDL by both decreasing the SR-A-dependent oxLDL uptake and increasing the ABCA1-dependent cholesterol efflux, without significant effect on other cholesterol transporters. Mechanistic experiments indicate that curcumin reduces SR-A expression via a ubiquitin/proteasome pathway. Furthermore, curcumin upregulated ABCA1 expression via $\mathrm{LXR} \alpha$-dependent transcriptional regulation (Zhao et al., 2012). Additional mechanisms, such as an activation of the AMPK/Sirt1/LXR $\alpha$ (Lin et al., 2015c) and the Nrf2/ HO-1 pathway (Kou et al., 2013) and inhibition of the p38MAPK pathway (Min et al., 2013) were also involved in its effects. These concerted mechanisms together contribute to the inhibitory effect on foam cell formation mediated by curcumin. Recently, several curcumin derivatives, such as demethoxycurcumin $(50 \mu \mathrm{M})$ (Kou et al., 2013) and nicotinate-curcumin (10 $\mu \mathrm{M})$ (Gu et al., 2016), have also been shown to inhibit THP-1 macrophagederived foam cell formation. In addition, curcumin functions as an inhibitor of PCSK9 and upregulates hepatic LDLR expression (Tai et al., 2014).

More importantly, curcumin (500-1000 mg/kg diet) attenuated the atherosclerosis development in $\mathrm{ApoE}^{-/-}$ mice by reducing the SR-A expression and increasing the ABCA1 expression in vivo (Hasan et al., 2014). The inhibitory activity against foam cell formation and the antiatherosclerotic effects of curcumin were also confirmed by several other studies (Kou et al., 2013; Min et al., 2013; Lin et al., 2015c; Soltani et al., 2017). Interestingly, curcumin lowered the level of LDL-C and TG in patients at risk for CVD (Qin et al., 2017). Therapeutic efficacy and molecular targets of curcumin in cardiovascular diseases have been reviewed elsewhere (Li et al., 2019).

3. Danshensu. Danshensu is an active component isolated from an eminent Chinese medicinal herb Salvia miltiorrhiza (Danshen), which is known for improving blood microcirculation. Danshen is widely used in China and other countries for treating various cardiovascular disorders, including atherosclerosis, angina pectoris, and myocardial infarction, due to potent anti-inflammatory, antioxidative, and lipid-lowering effects (Xu and Liu, 2013). It was shown that danshensu suppressed the LPS- and oxLDL-induced lipid accumulation in RAW 264.7 macrophages at the concentrations of 5-20 $\mu \mathrm{M}$ by activating LXR and inhibiting NF- $\kappa \mathrm{B}$, as well as the LPS-induced expression of fatty acid binding protein 4 and perilipin-2 (Xie et al., 2011; Wang et al., 2017c). Further studies also showed that danshensu (1-10 $\mu \mathrm{M})$ prevented cholesterol accumulation and foam cell formation in RAW264.7 mouse macrophages by inhibiting CD36-mediated lipid uptake, while enhancing ABCA1 (G1)-mediated cholesterol efflux (Wang et al., 2010b; Gao et al., 2016). In addition, danshensu could improve dyslipidemia by decreasing the levels of LDL-C and fatty acid by inhibiting HMG-CoA reductase and fatty acid synthase expression (Yang et al., 2011).

An earlier in vivo study has revealed that danshensu $(67.5 \mathrm{mg} / \mathrm{kg}$ body weight per day) attenuated high methionine-rich diet-induced accumulation of foam cells in rat aortic endothelium by suppressing the expression of TNF- $\alpha$ and intercellular adhesion molecule 1 (ICAM1) (Yang et al., 2010). Furthermore, Danshensu Bingpian Zhi (20 and $40 \mathrm{mg} / \mathrm{kg}$ body weight), which has the main bioactive constituent Danshensu, attenuated atherosclerosis in $\mathrm{ApoE}^{-/-}$mice.

4. 6-Dihydroparadol. Ginger (Zingiber officinale) is a common food-derived health supplement, used worldwide due to its potential preventive effects in multiple diseases, including atherosclerosis and other inflammatory disorders (Aktan et al., 2006). Gingerols are the main bioactive compounds from ginger with potent antiinflammatory properties in cultured cells. 6-Dihydroparadol, a bioactive metabolite of gingerols, displayed potent antiinflammatory effects by blocking the NF- $\kappa$ B/inducible $\mathrm{NO}$ synthase/NO pathway in LPS-stimulated macrophages (Aktan et al., 2006). 6-Dihydroparadol $(30 \mu \mathrm{M})$ was recently shown to promote cholesterol efflux from human THP-1 macrophages by increasing the expression of ABCA1 and ABCG1 via preventing the proteasome-dependent protein degradation, underscoring its therapeutic potential in preventing foam cell formation and possibly atherosclerosis (Wang et al., 2018a).

5. Paeonol. Paeonol is a bioactive phenolic compound isolated from traditional Chinese medicine Paeonia suffruticosa (Cortex Moutan), which is commonly used to treat inflammatory disorders including atherosclerosis (Zhao et al., 2013b). The antiatherosclerotic effects of paeonol have been well documented in several experimental animal models of atherosclerosis, including rabbits (Shi et al., 1988; Li et al., 2009a), quails (Dai et al., 1999), and $\mathrm{ApoE}^{-/-}$mice (Zhao et al., 2013b; Li et al., 2015c). Four major antiatherosclerotic mechanisms of paeonol include 1) endothelial protection (reducing monocyte adhesion to inflamed endothelium, endothelial apoptosis, and senescence) (Nizamutdinova et al., 2007; Pan and Dai, 2009; Wang et al., 2012d; Bao et al., 2013; Chen et al., 2013; Jamal et al., 2014; Liu et al., 2014b; Yuan et al., 2016); 2) inhibiting the proliferation and migration of VSMC (Chen et al., 2014; $\mathrm{Hu}$ et al., 2016a); 3) antiplatelet activation (Shi et al., 1988); and 4) anti-foam cell formation (Zhao et al., 2013b; Li et al., 2015c).

Studies performed in isolated macrophages from $\mathrm{ApoE}^{-1-}$ mice treated with paeonol (50 and $100 \mu \mathrm{g} / \mathrm{ml}$ ) showed that it significantly reduced the oxLDL-induced 
lipid accumulation, probably by promoting cholesterol efflux via activating the LXR $\alpha / A B C A 1$ pathway, which was suggested by study in J774.A1 macrophages (Zhao et al., 2013b). Silencing ABCA1 or LXR $\alpha$ abolished the paeonol-induced effects on cholesterol efflux and lipid accumulation, supporting the indispensable effect of LXR $\alpha$ and ABCA1 in mediating the protective effects. The inhibition of foam cell formation and antiatherosclerotic effects of paeonol were reproduced by another independent group, who confirmed the ABCA1activating effects of this compound (5, 10 and $50 \mu \mathrm{M})$ in RAW264.7 macrophages and suggested that the antifoam cell formation effects of paeonol also depended on CD36 inhibition and activation of HO-1 (Li et al., 2015c).

More importantly, paeonol treatment led to reduced atherosclerotic lesions formation and attenuated systemic inflammation, as well as increased ABCA1 expression in $\mathrm{ApoE}^{-/-}$mice (Zhao et al., 2013b). In addition to effects mentioned above, paeonol also reduced the levels of malondialdehyde and oxidized LDL in hyperlipidemia rats (Dai et al., 2000).

6. Polydatin. Polydatin represents one of the major bioactive ingredients from Rhizoma Polygoni Cuspidati (Da Huang), an eminent Chinese medicinal herb, which dispels dampness, alleviates jaundice, reduces fever, subsides toxins, and removes stasis (Du et al., 2009; Deng et al., 2011; Liu et al., 2012b; Ma et al., 2016; Gugliandolo et al., 2017). Pharmacological studies in the past decade have shown that polydatin exhibits a broad range of bioactivities with cardiovascular relevance, including antioxidation, anti-inflammation, cardioprotection, blood vessel dilation, lipid-lowering effect, as well as inhibitory effects on platelet aggregation, monocyte adhesion to activated endothelium, thrombus formation, and atherosclerosis development (Du et al., 2009; Deng et al., 2011; Liu et al., 2012b; Ma et al., 2016; Gugliandolo et al., 2017). The preventive effects of polydatin on foam cell formation were reported recently (Wu et al., 2015b). Polydatin treatment of 48 hours reduced the level of TC, FC, and CE, together with reducing the secretion of TNF- $\alpha$ and IL- $1 \beta$ in oxLDL-stimulated ApoE $\mathrm{E}^{-1-}$ mouse macrophages. The mechanism of these effects is linked to the activation of PPAR $\gamma$-dependent ABCA1 upregulation and the decrease of CD36 expression (Wu et al., 2015b). Polydatin also improved dyslipidemia via suppressing PCSK9 and upregulating hepatic LDLR expression ( $\mathrm{Li}$ et al., 2018a).

7. Protocatechuic Acid. Protocatechuic acid (PCA) is a bioactive compound present in some medicinal herbs such as Danshen ( $\mathrm{Li}$ et al., 2018d)). It is a major metabolite of fruit/vegetable-derived anthocyanins (such as C3G) generated by gut microflora (Hidalgo et al., 2012; Wang et al., 2012a; Lin et al., 2015b). Consumption of the PCA-rich vegetable chicory ( $5 \mathrm{~g} / \mathrm{kg}$ diet) reduced the TC and $\mathrm{CE}$ accumulation in isolated macrophages via upregulation of the ABCA1/ABCG1-mediated cholesterol efflux
(Lin et al., 2015b). Because of the fact that PCA is the major component of chicory, a later study found that PCA, rather than its parent drug C3G, promoted the ABCA1 (G1)-dependent cholesterol efflux from macrophages. miRNA microarray assay elucidated that PCA reduced the miRNA-10b expression, thereby upregulating the expression of ABCA1 (G1) in MPMs (Wang et al., 2012a). Protocatechuic acid could also regulate lipid metabolism via suppressing the expression of HMGCoA reductase (Liu et al., 2010b). In in vivo study, C3G (50 mg/kg body weight) induced RCT in the $\mathrm{ApoE}^{-/-}$ mice model and the atheroregression could be inhibited by antibiotic treatment, suggesting that the atheroprotective effect of $\mathrm{C} 3 \mathrm{G}$ is gut microbiota dependent (Wang et al., 2012a).

In addition to influence of cholesterol metabolism, PCA $(10,20$, and $40 \mu \mathrm{g} / \mathrm{ml})$ was also shown to inhibit ICAM1 and vascular cell adhesion molecule 1 (VCAM1)-dependent monocyte adhesion to activated HUVECs, as well as CCL2-mediated monocyte transmigration, thereby reducing the development of atherosclerosis in $\mathrm{ApoE}^{-1-}$ mice at the dose of $0.03 \mathrm{~g} / \mathrm{kg}$ (Wang et al., 2010a, 2011; Stumpf et al., 2013). PCA (150 $\mu \mathrm{g} / \mathrm{ml})$ also exhibited inhibitory effects on VSMC proliferation in A7r5 smooth muscle cell line (induced by oleic acid) by activating AMPK and arresting cell cycle at $G_{0} / G_{1}$ phase (Lin et al., 2015a). Also, PCA increased the endotheliumdependent vasorelaxation as well as tetrahydrobiopterin levels and combated eNOS uncoupling (Liu et al., 2016b).

8. Salicylic Acid. Aspirin (acetyl salicylic acid) is an anti-inflammatory drug used as a cardiovascular therapeutic. It (600 and $1200 \mu \mathrm{M}$ ) upregulated the expression of ABCA1 and SR-BI, thereby stimulating the HDL-mediated cholesterol efflux in THP-1 macrophages (Viñals et al., 2005; Lu et al., 2010). In light of the key role of AMPK in regulating lipid and energy metabolism, Fullerton et al. (2015) explored whether AMPK was involved in the induction of ABCA1 and ABCG1 expression by aspirin. The authors observed that AMPK $\beta 1$ deletion significantly impaired cholesterol efflux, without affecting lipid uptake in macrophages. Moreover, the AMPK activation by salicylate (salts and esters of salicylic acid) prevented foam cell formation via promoting $\mathrm{HDL}$ and ApoA-1-mediated cholesterol efflux by increasing the ABCA1 and ABCG1 expression in BMDMs. However, the preventive effect of salicylate was reduced in AMPK $\beta 1$-deficient BMDMs, indicating that the effect of salicylate on cholesterol efflux and foam cell formation is dependent on AMPK (Fullerton et al., 2015). Another study confirmed this result and observed that a low-dose of aspirin $(<0.5 \mathrm{mM})$ also upregulates the ABCA1dependent cholesterol efflux via activating the PPAR $\alpha$ pathway, which provides additional insights to explain the cardiovascular actions of aspirin (Wang et al., 2010e). In addition, aspirin attenuated atherosclerosis in $\mathrm{ApoE}^{-1-}$ mice partly by suppressing systemic 
inflammation and promoting inflammation resolution (Petri et al., 2017).

9. Salvianolic Acid B. Salvianolic acid B is a major hydrophilic constituent from Danshen, which has been demonstrated to exhibit antiatherosclerotic effects in a model of neointimal hyperplasia in rabbits (Yang et al., 2011) and in $\mathrm{ApoE}^{-I-}$ mice (Chen et al., 2006; Lin et al., 2007). Salvianolic acid B has shown to inhibit lipid peroxidation (LDL oxidation) (Yang et al., 2011) and activate AMPK (Cho et al., 2008). A high-throughput screening assay identified salvianolic acid B (15 and $30 \mu \mathrm{M})$ as an effective CD36 antagonist that blocks the oxLDL uptake in RAW264.7 macrophages (Wang et al., $2010 b)$. Further follow up studies showed that salvianolic acid B directly bound to CD36 $\left(K_{\mathrm{d}}=3.74 \mu \mathrm{M}\right)$ and inhibited the CD36 expression. By doing so, salvianolic acid $\mathrm{B}(1,10$, and $100 \mu \mathrm{M})$ reduced the CD36-dependent lipid uptake and foam cell formation in mouse macrophages, as well as in PMA-stimulated THP-1 human macrophages (Bao et al., 2012). Salvianolic acid B has been reported to promote the HDL- and ApoA-1mediated cholesterol efflux in differentiated THP-1 macrophages via inducing the expression of ABCA1 (Yue et al., 2015). Further mechanistic studies showed that the upregulation of ABCA1, promoted by salvianolic acid B ( 1 and $10 \mu \mathrm{M})$, was reversed by PPAR $\gamma$ and LXR $\alpha$ inhibitors. This evidence indicates that salvianolic acid $\mathrm{B}$ reduces lipid accumulation by promoting cholesterol efflux via a PPAR $\gamma / \mathrm{LXR} \alpha / \mathrm{ABCA} 1-$ dependent pathway in THP-1 macrophages (Yue et al., 2015).

10. Sesamol. Sesamol is an essential bioactive compound of sesame oil (Sesamum indicum), which is a cardiovascular protective dietary supplement. Previous studies have shown that a sesame oil aqueous extract $(0.75 \mathrm{mg} / \mathrm{mouse}$ per day) prevented or regressed atherosclerosis in $\mathrm{LDLR}^{-/-}$mice (Narasimhulu et al., 2018) and sesamol derivative (INV-403) (20 mg/kg per day) played a positive role in hyperlipidemic rabbits fed with an atherogenic diet (Ying et al., 2011). Mechanistic studies showed that both sesame oil and sesame oil aqueous extract slightly increased the ABCA1 expression, while decreasing the mRNA expression of CD36, CD68, SR-A, and LOX-1 in the aortic arch segments of $\mathrm{LDLR}^{-/-}$mice model (Narasimhulu et al., 2018).

Furthermore, it is possible that sesamol is mainly responsible for the observed atheroprotective effects of sesame oil. A recent study identified that both sesamol $(25-100 \mu \mathrm{M})$ and sesame oil (1-10 $\mu \mathrm{g} / \mathrm{ml})$ increase the expression/activity of PPAR $\gamma$ and $\operatorname{LXR} \alpha$ in Chinese hamster ovary cells via an MAPK-dependent mechanism. Most importantly, sesamol and sesame oil boosted cholesterol efflux from MPM (Majdalawieh and Ro, 2015). This evidence, together with a previous report showing that sesamol $(10,30$, and $100 \mu \mathrm{M})$ suppressed inflammatory response in LPS-stimulated RAW264.7 mouse macrophages (via AMPK-dependent NF- $\kappa \mathrm{B}$ suppression) (Wu et al., 2015d), collectively indicates that sesamol has the potential to reduce foam cell formation and atherogenesis (Majdalawieh and Ro, 2015).

11. Resveratrol. Resveratrol is a major stilbenoid compound isolated from red wine. Resveratrol displays reproducible antiatherosclerotic effects in several animal models, including $\mathrm{ApoE}^{-/-}$mice (Do et al., 2008; Chang et al., 2015), ApoE*3-Leiden CETP mice (Berbée et al., 2013), and Apo: ${ }^{-/-} / \mathrm{LDLR}^{-/-}$mice (Fukao et al., 2004). The atheroprotective mechanisms of resveratrol include inhibition of LDL oxidation (Berrougui et al., 2009), enhancement of endothelial protection, decrease of TMAO via gut microbiota (Chen et al., 2016), inhibition of the proliferation and migration of VSMCs, monocyte/macrophage differentiation, and platelet activation (Vasamsetti et al., 2016). It was reported that resveratrol $(2.5 \mu \mathrm{M})$ reduced the LPS-induced RAW264.7 foam cell formation via attenuating the NADPH oxidase 1 (Nox1)-dependent ROS production and MCP1 expression via Akt/Foxo3a and AMPK/Sirt1 pathways (Park et al., 2009a; Dong et al., 2014). Follow up studies in human macrophages identified that resveratrol at the concentrations of 10 or $100 \mu \mathrm{M}$ reduced oxLDL uptake in THP-1 macrophages (Voloshyna et al., 2013). Resveratrol promoted ApoA1- and HDL-mediated cholesterol efflux in both mouse (RAW264.7, J774.A1, MPMs) and human macrophages (THP-1) by increasing the expression of ABCA1 (Berrougui et al., 2009; Allen and Graham, 2012) and ABCG1 via PPARv/LXR $\alpha$ and adenosine 2A receptor pathway (Voloshyna et al., 2013). In addition to the therapeutic effects in foam cell formation induced by oxLDL, resveratrol $(10 \mu \mathrm{M})$ also ameliorated foam cell formation in J774.A1 macrophages caused by Chlamydia pneumonia infection by inhibiting the synthesis of IL-17A (Di Pietro et al., 2013). Clinical studies have shown that resveratrol lowers the level of TC and TG in patients with dyslipidemia (Simental-Mendia and Guerrero-Romero, 2019). Resveratrol also regulated lipid metabolism via inhibiting cholesterol-ester-transport protein and HMG-CoA expression/level (Cho et al., 2008).

12. Epigallocatechin Gallate. EGCG is the most studied polyphenol (catechin), derived from tea and possessing antiatherosclerotic and plaque-stabilizing effects in rats, rabbits, and $\mathrm{ApoE}^{-/-}$mice (Chyu et al., 2004; Xu et al., 2014a; Wang et al., 2018e,f). Previous studies have shown that EGCG $(25 \mu \mathrm{M})$ attenuated the oxLDL-induced apoptosis of HUVECs by blocking JNK activation (Choi et al., 2008). Furthermore, EGCG (40 and $80 \mu \mathrm{g} / \mathrm{ml}$ ) reversed the TNF- $\alpha$-induced ABCA1 downregulation and reduced the cholesterol efflux from THP-1 macrophages by activating the Nrf2-dependent NF- $\kappa$ B inhibitory effects (Jiang et al., 2012). In the same cell line, EGCG $(10 \mu \mathrm{M})$ also blocked the oxLDL-induced upregulation of SR-A, thus blocking the oxLDL uptake and foam cell formation (Chen et al., 2017). Mechanistic studies also showed that EGCG is having a preventing 
action on hyperlipidemia by increasing the expression and activity of LDLR (Lee et al., 2008).

\section{Phenylpropanoids}

Phenylpropanoids are diverse natural aromatic products comprising a hydroxy- and/or alkoxy-substituted aromatic phenyl moiety and a three-carbon propene tail of coumaric acid, and the key intermediate in the biosynthesis of phenylpropanoid-derived plant compounds (Barros et al., 2016). These compounds have a wide variety of biologic activities, including antimicrobial, antitumor, anti-inflammatory activities, and a cholesterol-lowering effect (Okonkwo et al., 2016).

1. Lignans. Lignans represent a class of natural polyphenols, which are dimers derived from two molecules of a phenylpropanoid derivative (a C6-C3 monomer). They are present in flaxseed, sesame, soybeans, and some fruits. By acting as phytoestrogens, dietary lignans exhibit potent antiviral, antioxidative, antitumor, and antiatherosclerotic activities (Peterson et al., 2010).

a. Arctigenin. Arctigenin, a phenylpropanoid dibenzylbutyrolactone lignin, has many beneficial biologic effects, such as immune modulation and regulation of metabolic disorders (Huang et al., 2012a; He et al., 2018). In oxLDL-loaded THP-1 macrophages, the expression of ApoE, ABCA1, and ABCG1 were increased by arctigenin (50 and $100 \mu \mathrm{M})$, resulting in promoting cholesterol efflux (Xu et al., 2013d). Arctigenin has no obvious effects on the expression of SRs, such as SR-BI, SR-A1, and CD36 (Xu et al., 2013d). Further studies indicated that arctigenin $(200 \mathrm{mg} / \mathrm{kg}$ body weight per day) decreased the cholesterol levels without altering serum TG and adiponectin levels in mice (Huang et al., 2012a), and suppressed the lipid accumulation and body weight gain in HFD-induced obese mice (Han et al., 2016).

b. Leoligin. Leoligin, the major lignan of the alpine flower Edelweiss (Leontopodium alpinum Cass.), has shown obvious antihyperplastic effects and regulatory activity of lipoprotein metabolism through interference with CETP (Reisinger et al., 2009; Duwensee et al., 2011). Recently, it was reported that leoligin reduced LDL-C levels and postprandial serum glucose peaks due to the direct inhibition of 3-hydroxy-3-methylglutaryl-CoA reductase and moderated PPAR $\gamma$ agonistic activity in $\mathrm{ApoE}^{-/-}$mice (Scharinger et al., 2016). Additionally, it (3-20 $\mu \mathrm{M})$ increased cholesterol efflux from THP-1 macrophages by upregulating both protein and mRNA levels of the ABCA1 and ABCG1 (Wang et al., 2016a). These novel activities suggest that leoligin may be of promise to be further studied as a therapeutic agent for preventing the formation of foam cells.

c. Sesamin. Sesamin is a phytochemical that possesses diverse bioactivities, such as prevention of fat storage, decrease of cholesterol in serum, antiinflammatory, and antioxidative activities (Lee et al., 2009c; Rogi et al., 2011). It was shown that sesamin (0.1, 1, and $10 \mu \mathrm{M})$ inhibited the oxLDL-induced cholesterol accumulation and enhanced cholesterol efflux from RAW264.7 macrophages, possibly via an upregulation of PPAR $\gamma, \mathrm{LXR} \alpha$, and ABCG1 (Liu et al., 2014a). This study also indicated that PPAR $\gamma$ played an essential role in sesamin-mediated cholesterol efflux, since a PPAR $\gamma$ antagonist (GW9662) could abolish the cholesterol efflux-promoting effect of sesamin. Furthermore, sesamin increased the PPAR $\gamma$ and LXR $\alpha$ transcriptional activity in a concentration- and time-dependent manner via MAPK signaling (Majdalawieh and Ro, 2014). In vivo studies showed that sesamin attenuated atherosclerosis in $\mathrm{ApoE}^{-/-}$mice by suppressing vascular inflammation (Wu et al., 2010). The lipid-lowering effect of sesamin was exerted through promoting the fecal excretion of sterols and inhibiting HMG-CoA reductase (Liang et al., 2015).

d. Honokiol. Honokiol is a biphenolic natural product, which is present in the traditional Chinese herbal medicine Magnolia bark. During the last decades, Magnolia bark has been used as an analgesic to treat anxiety and mood disorders (Lee et al., 2011; Sarris et al., 2013). With more and more biologic activities being discovered, this neolignan is also being extensively studied for its therapeutic potential in atherosclerosis. It was found that honokiol $(30 \mu \mathrm{M})$ was capable of activating the RXR/LXR heterodimer in RAW264.7 cells, resulting in the induction of ABCA1 expression and enhancement of cholesterol efflux from MPMs (Kotani et al., 2010). Consistent with these data, another study showed that honokiol increased ABCA1 expression by binding to $\mathrm{RXR} \beta$. It also increased the ABCG1 and ApoE expression in THP-1 macrophages (Jung et al., 2010). Honokiol and the structurally related neolignan magnolol have also been characterized as dual ligands for the transcriptional activity of the RXR/PPAR $\gamma$ dimer (Fakhrudin et al., 2010; Atanasov et al., 2013; Wang et al., 2014b). It has been also shown that hepatic PPAR $\gamma$ and its target genes could be upregulated by this neolignan at $10 \mu \mathrm{M}$ (Zhong and Liu, 2018).

2. $\alpha$-Asarone. As a major active constituent of Acorus tatarinowii Schott, $\alpha$-asarone exhibits a wide range of bioactivities. It was observed that purple perilla extract with $\alpha$-asarone increased the expression of ABCA1 and ABCG1 and accelerated the cholesterol efflux from lipid-loaded J774A.1 macrophages. It also enhanced the expression of LXR $\alpha$ and PPAR $\gamma$ in vitro (Park et al., 2015). These data indicated that $\alpha$-asarone promoted the macrophage cholesterol efflux through the PPAR $\gamma$-LXR $\alpha$-ABC transporters pathway.

In terms of lipid metabolism, a previous study has shown that there is an association between the intake of $\alpha$-asarone ( $80 \mathrm{mg} / \mathrm{kg}$ body weight per day) and the decreased level of serum cholesterol in hypercholesterolemic rats by inhibition of HMG-CoA reductase (Rodríguez-Páez 
et al., 2003). Another animal study revealed that 2,4,5trimethoxycinnamic acid, the major and nontoxic metabolite of $\alpha$-asarone, had most of the pharmacological properties of $\alpha$-asarone. Both compounds lowered TC, LDL-C, and HDL-C in hypercholesterolemic rats (Antunez-Solis et al., 2009).

3. Chlorogenic Acid. Chlorogenic acid, a naturally occurring phenolic acid present in coffee, as well as in the leaves and fruits of diverse dicotyledonous plants, acts as an important intermediate of lignin biosynthesis. Chlorogenic acid exhibited various effects, like antioxidative activity and modulation of blood glucose and cholesterol metabolism (Rodriguez de Sotillo and Hadley, 2002; Tošović et al., 2017). In vivo, it reduced (at 200 and $400 \mathrm{mg} / \mathrm{kg}$ body weight per day) the percentage and the total atherosclerotic lesion area and aortic dilatation in cholesterol-rich diet-fed $\mathrm{ApoE}^{-1-}$ mice, as well as decreased levels of TC, LDL-C, and TG in serum (Wu et al., 2014).

Recent reports suggested that chlorogenic acid also displays a modulatory effect on foam cell formation. Chlorogenic acid ( 1 and $10 \mu \mathrm{M})$ inhibited foam cell formation and decreased the oxLDL-elicited neutral lipid and cholesterol accumulation in RAW264.7 macrophages via increasing the transcription of PPAR $\gamma$, LXR $\alpha$, ABCA1, and ABCG1 (Wu et al., 2014). Interestingly, the serum containing chlorogenic acid metabolites inhibited the oxLDL-induced lipid accumulation and increased the cholesterol efflux in RAW264.7 cells. Furthermore, five serum metabolites of chlorogenic acid were tested and the results showed that caffeic, ferulic, and gallic acids significantly increased the HDL-mediated cholesterol efflux from RAW264.7 cells (Wu et al., 2014). These data suggest that these metabolites might be potential bioactive compound, accounting for the in vivo effect of chlorogenic acid. In addition, it is likely that other mechanisms are involved in the lipid regulation by this natural product. In this line, it was reported that chlorogenic acid $(30 \mu \mathrm{M})$ promoted the efflux of TC and triacylglycerol and increased mRNA expression of ABCA1, CYP7A1, and AMPK $\alpha 2$ in HepG2 cells (Hao et al., 2016).

4. Caffeic and Ferulic Acid. Caffeic acid is a wellknown phenolic phytochemical present in coffee and diverse other plants, since it represents a key intermediate in the biosynthesis of lignin. The closely related ferulic acid is a hydroxycinnamic acid formed by the conversion of caffeic acid and it is also present in the cell walls of diverse plants. Both caffeic acid and ferulic acid exhibit anticancer, antioxidative, and diverse other biologic activities (Sato et al., 2011; Rosendahl et al., 2015). Both compounds are being extensively studied for their potential benefits in various disorders, such as inflammation, neurodegenerative diseases, cancer, CVDs, and atherosclerosis (Zhao and Moghadasian, 2008).
It was reported that caffeic and ferulic acid, the major phenolic acids of coffee, enhanced HDL-mediated cholesterol efflux, but not the ApoA-1-mediated efflux in THP-1 macrophages. Both compounds $(1 \mu \mathrm{M})$ increased the expression of ABCG1 and SR-BI, but not ABCA1 (Uto-Kondo et al., 2010). Further experiments showed that caffeic acid was identified as a PPAR $\alpha$ agonist in vitro (Kim et al., 2014). As a metabolite of chlorogenic acid, ferulic acid $(0.25,0.5$, and $1 \mu \mathrm{M})$ has also been shown to have an enhancement effect on HDL-mediated cholesterol efflux from macrophages through increasing the expression of ABCG1 and SR-BI (Uto-Kondo et al., 2010). Furthermore, it was demonstrated that ferulic acid $(1 \mu \mathrm{M})$ displays antiatherosclerotic potential by increasing the ABCA1 and ABCG1 expression in macrophage form cells and further promoting cholesterol efflux (Chen and Wang, 2015). In this light, counteraction of foam cell formation can be also considered in relation to the potential antiatherogenic properties of phenylpropanoids, in addition to their antioxidative/anti-inflammatory functions.

\section{E. Alkaloids}

1. Arecoline. Arecoline is a nicotinic acid-based bioactive alkaloid isolated from areca nut, which has a medicinal potential in the treatment of neurodegenerative diseases (Ghelardini et al., 2001). Cardiovascular benefits of arecoline are largely unknown. In oxLDLstimulated macrophages, arecoline reduced the cholesterol accumulation in a dose-dependent manner by reducing $\mathrm{TC}, \mathrm{FC}$, and $\mathrm{CE}$. In addition, arecoline also promoted the cholesterol efflux by inducing ABCA1 expression. Further studies were warranted to evaluate the effects of arecoline on lipid uptake in RAW264.7 macrophage and its therapeutic effect on atherosclerosis in vivo (Ouyang et al., 2012). In vivo study showed that arecoline suppressed atherosclerosis in $\mathrm{ApoE}^{-/-}$ mice by inhibiting NF- $\kappa \mathrm{B}$ activation (Zhou et al., 2014).

2. Berberine. Berberine is a bioactive alkaloid isolated from several medicinal plants, including Berberis (Huang Lian). Berberine-containing plants have been used in China for a long time to treat various disorders, including CVD (Feng et al., 2019). Berberine protects against atherosclerosis in various animal models due to its lipid-modulating effects. Two well-elucidated molecular targets of berberine in modulating lipid levels are LDLR (Kong et al., 2004) and proprotein convertase subtilisin/kexin type 9 (PCSK9) (Dong et al., 2015). In 2010, Lee et al. (2010) observed that berberine $(5,10$, and $20 \mu \mathrm{M}$ ) inhibited THP-1 macrophage foam cell formation by promoting LXR $\alpha /$ ABCA1-dependent cholesterol efflux. However, berberine did not affect ABCG1, SR-BI, CD36, and SR-A. Further studies indicate that berberine ( 5 and $10 \mathrm{mg} / \mathrm{l}$ ) also prevented the oxLDL-induced upregulation of LOX-1 and downregulation of SR-BI in THP-1 macrophages, without affecting the SR-A and ABCA1 expression (Guan et al., 2010; 
Chi et al., 2014a). In addition, several derivatives of berberine, such as 13-hexylberberine (Li et al., 2010), were shown to act as potential CD36 inhibitors. Possible inhibition of foam cell formation and antiatherosclerotic potential of these derivatives needs to be further evaluated in vitro and in vivo. Other mechanisms linked to the effect of berberine on foam cell formation include activation of AMPK/Sirt1 (Chi et al., 2014b), autophagy induction (Kou et al., 2017), as well as inhibition of adipocyte enhancer-binding protein 1 (Huang et al., 2012b).

However, a contradictory study reported that berberine $(5 \mathrm{mg} / \mathrm{kg}$ per day) unexpectedly promoted atherosclerosis in mice by enhancing the SR-A-mediated oxLDL uptake and foam cell formation in human and mouse macrophages ( $\mathrm{Li}$ et al., 2009b). Mechanistic studies revealed that berberine-mediated SR-A upregulation was exerted through suppressing the phosphatase and tensin homolog expression, thus promoting the activation of Akt in RAW264.7 macrophages ( $\mathrm{Li}$ et al., 2009b). The discrepancy of the atherosclerosismodulating effects of berberine may be due to different animal models and doses, which were used in these studies. Despite most of the literature indicating that berberine has beneficial effects in cardiovascular and metabolic diseases, the precise effects and mechanisms of berberine action in the modulation of foam cell formation need to be further evaluated. In patients with dyslipidemia, berberine reduces TC, LDL-C, TG and increases HDL-C, partially through inhibiting PCSK9 and increasing LDLR expression/activity (Kong et al., 2004; Cameron et al., 2008; Ju et al., 2018).

3. Piperine. Piperine is a biologically active ingredient isolated from the fruits of black pepper (Piper nigrum). It has been shown that piperine $(100 \mu \mathrm{g} / \mathrm{ml})$ decreases cholesterol uptake dose dependently in Caco2 cells by reducing the levels of the membrane localized cholesterol transporter protein-Niemann-Pick disease, type C1 (NPC1) like intracellular cholesterol transporter 1 (NPC1L1) and SR-BI (Duangjai et al., 2013). More recently, piperine $(25,50$, and $100 \mu \mathrm{M})$ was also found to promote the ABCA1 protein expression in THP-1-differentiated human macrophages, without affecting the ABCG1 and SR-BI expression (Wang et al., 2017d). Further studies revealed that piperine did not affect the gene expression of ABCA1, but increased the ABCA1 protein stability by preventing calpain-mediated ABCA1 protein degradation (Wang et al., 2017d). In addition, piperine regulated lipid metabolism via increasing hepatic LDLR expression via proteolytic activation of SREBPs in HepG2 cells (Ochiai et al., 2015). These pharmacological effects placed piperine as a promising food-derived bioactive compound with potential therapeutic implications in atherosclerosis.

4. Rutaecarpine. Rutaecarpine (or rutecarpine) is a nonbasic alkaloid isolated from the unripe fruits of the medicinal herb Evodia rutaecarpa (Wu Zhu Yu), which has been used in treating cardiovascular and cerebrovascular diseases (Tian et al., 2019b). A high-throughput screening assay for ABCA1 upregulators identified rutaecarpine as a potential active compound that increased the ABCA1 promotor activity in HepG2 cells $\left(\mathrm{EC}_{50}=0.27 \mu \mathrm{M}\right)(\mathrm{Xu}$ et al., 2014b). Further studies showed that rutaecarpine upregulated the expression of ABCA1 and SR-BI (without affecting ABCG1 and CD36) via $\operatorname{LXR} \alpha$ and $\operatorname{LXR} \beta$, thereby reducing the lipid accumulation and foam cell formation via promoting cholesterol efflux in vitro (RAW264.7 macrophages and HepG2 cells) and in vivo (ApoE $\mathrm{E}^{-/-}$mouse). By this mechanism, rutaecarpine $(20 \mathrm{mg} / \mathrm{kg}$ body weight per day) reduced atherosclerotic plaque development in $\mathrm{ApoE}^{-1-}$ mice. The atheroprotective effects of rutacarpine are accompanied by reduced macrophage and lipid content in atherosclerotic plaques (Xu et al., 2014b). Rutaecarpine lowered the level of TC, TG, LDL-C, and hs-CRP in hyperlipidemic and hyperglycemic rats via AMPK activation and $\mathrm{NF}-\kappa \mathrm{B}$ inhibition (Nie et al., 2016; Tian et al., 2019b). Several derivatives of rutaecarpine also exhibited antiatherosclerotic potential by enhancing cholesterol efflux from RAW264.7 macrophages to HDL and thus inhibiting foam cell formation ( $\mathrm{Li}$ et al., 2014).

5. Evodiamine. Evodiamine, an indoloquinazoline alkaloid, is present in the traditional Chinese medicine Fructus Evodiae (Chinese name: Wuzhuyu) (Shoji et al., 1986; Wagner et al., 2011). Evodiamine $(3-20 \mu \mathrm{M})$ increased cholesterol efflux from THP-1-derived human macrophages significantly by directly binding to ABCA 1 and thereby increasing ABCA1 stability and protein level (Wang et al., 2018c). Moreover, treatment of evodiamine (10 mg/kg body weight) for 4 weeks decreased the size of atherosclerotic lesions and alleviated the hyperlipidemia, as well as hepatic macrovesicular steatosis in $\mathrm{ApoE}^{-/-}$mice, probably through transient receptor potential vanilloid type 1 (TRPV1) pathway (Su et al., 2014).

6. Leonurine. Leonurine is an anti-inflammatory, antioxidative, and antiatherosclerotic pseudoalkaloid isolated from Herba leonuri (Jiang et al., 2017a). Recently, Jiang et al. (2017a) have demonstrated that leonurine $(5-80 \mu \mathrm{M})$ dose dependently inhibited the lipid accumulation (TC, FC, and CE) and foam cell formation in oxLDL-stimulated THP-1 macrophages. Mechanistic investigations indicated that leonurine inhibited foam cell formation by promoting ApoA-1and HDL-mediated cholesterol efflux via the PPAR $\gamma /$ LXR $\alpha / A B C A 1$ (G1) pathway (Jiang et al., 2017a). In this line, leonurine also increased the expression of key proteins in cholesterol efflux, including $\operatorname{PPAR} \gamma, \operatorname{LXR} \alpha$, ABCA1 (G1), and reduced atherosclerotic development in $\mathrm{ApoE}^{-/-}$mice fed with an atherogenic diet $(10 \mathrm{mg} / \mathrm{kg}$ body weight per day). More studies are warranted to study potential effects of leonurine on suppressing lipid uptake and the related mechanisms. 


\section{F. Steroids}

1. Diosgenin. Diosgenin and its glycoside form dioscin are pharmacologically active steroidal sapogenins present in Dioscorea plant species. Both diosgenin and dioscin have been shown to exhibit antioxidative, hypolipidemic, antithrombotic, and endothelial-protective effects (Son et al., 2007; Gong et al., 2011; Liu et al., 2012a; Lv et al., 2015; Wu et al., 2015c; Wang et al., 2017g). Due to their structural similarity to cholesterol, diosgenin and dioscin were used in industrial production of steroidal drugs and are known to be increasing cholesterol secretion as well as inhibiting cholesterol absorption (Son et al., 2007). Diosgenin displayed antiapoptotic effects against $\mathrm{H}_{2} \mathrm{O}_{2}$-induced apoptosis (Gong et al., 2010), as well as antiinflammatory effects in VSMCs by inhibiting the MAPK/ Akt/NF- $\kappa$ B pathway (Choi et al., 2010). Diosgenin $(14 \mu \mathrm{M})$ also blocked atherosclerosis by inhibiting the nuclear translocation of notch intracellular domain in THP-1 cells (Binesh et al., 2018). It also inhibited the TNF- $\alpha$-induced leukocyte adhesion to activated endothelial cells by inhibiting the upregulation of ICAM1, VCAM1, and endothelial lipase (Wu et al., 2015c). In addition, dioscin (1-4 $\mu \mathrm{M}$ ) inhibited oxLDL uptake by blocking systemic inflammation and the LOX-1/NF- $\kappa$ B pathway in MPMs from atherosclerotic rats (Wang et al., 2017g). Also, diosgenin promoted cholesterol efflux by increasing the ABCA1 expression, independent of $\mathrm{LXR} \alpha$ ( $\mathrm{Lv}$ et al., 2015). The potential mechanism of diosgenin $(10-80 \mu \mathrm{M})$ in reducing foam cell formation in THP-1 macrophages as well as in atherogenesis is proposed to be through inhibiting of miRNA-19b (Lv et al., 2015).

2. Fucosterol. Fucosterol is a sterol that is present in marine algae. It has beneficial effects in hypercholesterolemia, due to its activity to reduce LDL-C and increase HDL-C (Hoang et al., 2012). A recent study (Hoang et al., 2012) has shown that fucosterol is a dual LXR $\alpha / \beta$ agonist that promotes cholesterol efflux from THP-1 macrophages at 100 and $200 \mu \mathrm{M}$, by increasing the expression of efflux transporters-ABCA1, ABCG1, as well as ApoE; meanwhile, it also reduces the intestinal NPC1L1-mediated cholesterol absorption. This is a beneficial effect, as it can avoid the side effects associated with the LXR activation, probably due to upregulating Insig-2a, which is a negative regulator of the lipogenic transcription factor SREBP-1c (Hoang et al., 2012).

3. Panax Notoginseng Saponins. PNS are the major bioactive ingredients of the medicinal herb $P$. notoginseng (Duan et al., 2017). PNS have extensive cardiovascular protective effects, including preventing endothelial dysfunction and increasing blood flow, antioxidation, anti-inflammation, antithrombosis, inhibition of foam cell formation, and regulation of cardiac function (Jia et al., 2010; Yuan et al., 2011). PNS (40 and $80 \mathrm{mg} / \mathrm{l}$ ) decreased the accumulation of cholesterol esters via increasing the ABCA1 expression in macrophages (Jia et al., 2010). In vivo study shows that PNS inhibited foam cell formation in zymosan A-induced atherosclerosis rats at the dose of $100 \mathrm{mg} / \mathrm{kg}$ per day (Yuan et al., 2011). Recently, ginsengoside Rd, a purified constituent from PNS, also demonstrated antiatherosclerotic effects in $\mathrm{ApoE}^{-/-}$fed with atherogenic diet by attenuating foam cell formation ( $\mathrm{Li}$ et al., 2011). Mechanistic studies indicated that ginsengoside $\mathrm{Rd}(20 \mu \mathrm{M})$ blocked SR-Amediated oxLDL uptake via blocking voltage-independent $\mathrm{Ca}^{2+}$ channels in RAW264.7 cells (Li et al., 2011). It remains to be elucidated whether other saponins from PNS have protective effects against foam cell formation and may contribute to the atheroprotective effects of PNS.

4. Vitamin D. Vitamin D deficiency is associated with an increased risk of CVDs, such as hypertension and atherosclerosis (Weng et al., 2013). A landmark study in 2009 showed that deficiency of vitamin D promoted the modified LDL-induced foam cell formation of macrophages from patients with diabetes $(\mathrm{Oh}$ et al., 2009). Similarly, deficiency of macrophage vitamin D aggravated CD36 and SR-A-mediated lipid uptake (via JNK activation), as well as promoted insulin resistance to increase atherosclerosis in mice (Oh et al., 2015). Vitamin D deficiency-induced hypertension and atherosclerosis in $\mathrm{LDLR}^{-1-}$ mice were reversed by JNK2 deficiency (Oh et al., 2018). A recent study has shown that vitamin $\mathrm{D}$ deficiency decreased the HDL level and LXR/ABCA1(G1) expression, thus augmenting cholesterol accumulation and atherosclerosis in hypercholesterolemic microswine (Yin et al., 2015). On the other side, vitamin $D$ supplementation inhibited the CD36 and SR-A-mediated lipid (oxLDL and ac-LDL) uptake and promoted the nascent HDL generation in HepG2 cells via ABCA1-dependent cholesterol efflux. The final outcome of these effects is to reduce foam cell formation in vitamin D-treated cells (Oh et al., 2009; Yin et al., 2015). Vitamin D supplementation improves glycemic control, increases HDL-C, and decreases hsCRP levels in patients with CVD (Ostadmohammadi et al., 2019).

\section{G. Fatty Acids}

1. Docosahexaenoic Acid and Eicosapentaenoic Acid. Increasing evidence has shown that the consumption of fish oil and its main bioactive components EPA and DHA leads to a reduced risk of CVD. One major mechanism of the cardiovascular effects of fish oil is modulation of lipid homeostasis and foam cell formation (McLaren et al., 2011b). Studies observed that free polyunsaturated fatty acids buffer including DHA and EPA at $5 \mu \mathrm{M}$ significantly reduced the CD36 expression in human U937 monocytes (Pietsch et al., 1995). Furthermore, DHA and EPA $(25,50$, and $100 \mu \mathrm{M})$ inhibited acLDL uptake in human THP-1 macrophages partially through reduction of mRNA and protein expression of CD36 and SR-A. Other SR-independent mechanisms include reduction of macropinocytosis and 
expression of syndecan-4, which is involved in the uptake of other forms of modified LDL (McLaren et al., 2011b). Moreover, an earlier study in 1992 showed that EPA $(100,300 \mathrm{mg} / \mathrm{kg}$ body weight per day) inhibits the $\mathrm{CE}$ accumulation in macrophages by decreasing the expression of receptors of acLDL in Wistar rats (Saito et al., 1992). These studies underscore the preventive and therapeutic potential of EPA and DHA in atherosclerosis.

2.

13-Hydroxyoctadecadienoic

Acid. 13-Hydroxyoctadecadienoic acid (also known as 13HODE, 13-hydroxy linoleic acid) is a major oxidized lipid component of oxLDL and a natural and endogenous PPAR agonist (Nagy et al., 1998; Kämmerer et al., 2011). Treatment with 13-hydroxyoctadecadienoic acid (1 and $2.5 \mu \mathrm{M}$ ), but not with linoleic acid, increased the transactivation activity of PPAR, and downstream targets of PPAR/LXR $\alpha$ pathway, including ABCA1 (G1) and SR-BI. By doing so, 13-HODE (2.5 $\mu \mathrm{M})$ promoted ApoA-1-mediated cholesterol efflux in RAW264.7 macrophages, thus decreasing cellular cholesterol level and foam cell formation (Kämmerer et al., 2011).

3. Linoleic Acid. Dietary supplementation of isomers of conjugated linoleic acids has been known for a long time to promote the regression of pre-established atherosclerotic plaques (Mooney et al., 2012; Song et al., 2013). These compounds conferred atheroprotection mainly by functioning as endogenous activators of PPAR $\alpha, \operatorname{PPAR} \gamma$, and PPARG coactivator 1 alpha (PGC1 $\alpha$ ) (Ringseis et al., 2008). Conjugated linoleic acids isomers, such as c9t11-CLA $(50 \mu \mathrm{M})$ and t10c12-CLA $(50 \mu \mathrm{M})$, were shown to stimulate the ApoA-1-mediated cholesterol efflux, thus reducing lipid accumulation by increasing the expression of NPC-1, NPC-2, ABCA1, and LXR $\alpha$ (Ringseis et al., 2008; Reza et al., 2009). In addition, $1 \%$ conjugated linoleic acid blend (80:20 cis9,trans-11-CLA:trans-10,cis-12-CLA) also induced the regression of pre-established atherosclerotic plaques in $\mathrm{ApoE}^{-/-}$mice by promoting macrophage polarization toward a M2 anti-inflammatory phenotype (McCarthy et al., 2013). Published literature suggests that linoleic acid could possibly reduce TC via increasing the hepatic LDLR expression and activity (Ringseis et al., 2006).

\section{H. Amino Acids}

1. $L$-(+)-Citrulline. In the NO cycle, $\mathrm{L}-(+)$-citrulline is a side product of the eNOS-mediated NO production which uses L-arginine as a substrate. $\mathrm{L}-(+)$-Citrulline reduces endothelial senescence, VSMC proliferation, and atherosclerosis in several animal models, partially through increasing the NO bioavailability (Ruiz et al., 1999; Hayashi et al., 2005, 2006; Morita et al., 2014; Tsuboi et al., 2018). Citrulline ( $1 \mathrm{mM})$ also increased the ABCA1 and ABCG1 expression in differentiated THP-1 macrophages, thereby promoting HDL- and ApoA-1mediated cholesterol efflux (Uto-Kondo et al., 2014). Of clinical relevance, citrulline consumption (3.2 g/day for
1 week) increased the level of citrulline and arginine post citrulline sera and promoted the HDL- and ApoA-1mediated cholesterol efflux by increasing the expression of both ABCA1 and ABCG1 in BMDM (Uto-Kondo et al., 2014). This finding provides additional support for citrulline consumption conferring antiatherogenic effects by regulating cholesterol homeostasis (UtoKondo et al., 2014).

2. S-Allyl Cysteine. S-allyl cysteine is the most abundant bioactive compound from aged garlic extract. $S$-allyl cysteine exhibits potent antioxidative and antiinflammatory effects by inhibiting LDL oxidation and $\mathrm{NF}-\kappa \mathrm{B}$ activation and by counteracting atherogenic events, including foam cell formation (Ho et al., 2001). Furthermore, $S$-allyl cysteine (10, 20, and $40 \mathrm{mM}$ ) can increase the ABCA1 expression in differentiated THP-1 macrophages, indicating a potential to reduce lipid accumulation by promoting cholesterol efflux (MalekpourDehkordi et al., 2013).

\section{Carbohydrates}

1. Phellinus Linteus Polysaccharides. Phellinus linteus polysaccharide extracts (PLPEs) have immunomodulatory effects. In lipid-laden THP-1 macrophages, low concentration of PLPEs (from 5 to $20 \mu \mathrm{g} / \mathrm{ml}$ ) promoted ApoA-1-mediated cholesterol efflux by upregulating PPAR $\gamma / \mathrm{ABCA} 1(\mathrm{G} 1)$ in dose-dependent manner. However, high concentration of PLPEs (up to $100 \mu \mathrm{g} / \mathrm{ml}$ ) inhibited ApoA-1-mediated cholesterol efflux, increased the NADPH oxidase-dependent ROS production, and decreased the mitochondrial membrane potential and ATP release (Li et al., 2015d). Thus, the dose is an important consideration for future studies with experimental animals or human patients.

2. Astragalus Polysaccharides. Astragalus polysaccharides (APS) represent the polysaccharide fraction of the medicinal herb Astragalus membranaceus. APS (from 25 to $100 \mu \mathrm{g} / \mathrm{ml}$ ) has been shown to promote the ABCA1 expression in foam cells exposed to TNF- $\alpha$. As a consequence, APS promoted the cholesterol efflux and affected lipid accumulation (Wang et al., 2010d). Further studies indicate that APS $(100 \mu \mathrm{g} / \mathrm{ml})$ reversed TNF- $\alpha$-induced NF- $\kappa \mathrm{B}$ activation in THP-1-derived foam cells. The antiatherosclerotic properties of APS and their molecular mechanisms of action remain to be further elucidated (Wang et al., 2010d).

\section{J. Others}

1. Organosulfur Compounds: Allicin. Allicin is a sulfur-containing compound present in garlic, which has been shown to inhibit cholesterol synthesis in modified liver homogenates (Sendl et al., 1992). It also has inhibitory effects on inducible NO synthase expression in LPS-stimulated mouse macrophages (Dirsch et al., 1998). Moreover, allicin inhibited the LDL oxidation. The lipid-lowering, anti-inflammatory, and antioxidative properties are underlying its atheroprotective 
effects observed in hyperlipidemic mice and rabbits (Abramovitz et al., 1999; Gonen et al., 2005; Yokoyama et al., 2012). Importantly, allicin ( $40 \mathrm{mg} 3$ times daily for 12 weeks) also decreased the carotid intima/media thickness in CAD patients with hyperhomocysteinemia (Liu et al., 2017a). A recent study (Lin et al., 2017) also showed that allicin reduced the TC, FC, and CE levels in THP-1 macrophage-derived foam cells by upregulating the ABCA1-dependent cholesterol efflux via PPAR $\gamma /$ LXR $\alpha$ signaling pathway.

2. Pyranone Derivatives: Asperlin. Asperlin is a natural compound from the marine fungus Aspergillus versicolor LZD4403, which has potent anti-inflammatory activity (Zhou et al., 2017). A recent study showed that asperlin $(1-10 \mu \mathrm{M})$ has specific protective effects against LPS-induced foam cell formation by promoting cholesterol efflux from RAW264.7 mouse macrophages (Zhou et al., 2017).

3. Anthraquinone Derivatives: Emodin. Emodin is a pharmacologically active compound isolated from the roots of Rheum palmatum (Da Huang). A recent study showed that emodin (5 and $10 \mu \mathrm{M})$ promoted ApoA-1mediated cholesterol efflux from THP-1 macrophages via PPAR $\gamma / \mathrm{LXR} \alpha / \mathrm{ABCA} 1$ (G1) signaling pathway (Zhou et al., 2008; Fu et al., 2014) and thereby ameliorated diet-induced atherosclerosis in rabbits at the dose of $10 \mathrm{mg} / \mathrm{kg}$ body weight (Hei et al., 2006). Emodin was also reported to reduce atherosclerosis in $\mathrm{ApoE}^{-1-}$ mice (Zhou et al., 2008) and rabbits (Hei et al., 2006). In addition, emodin lowered blood glucose, TC, and TG in diabetic and hyperlipidemic rats (Zhao et al., 2009) by inhibiting SREBP-1 and SREBP-2 (Li et al., 2016).

4. Polyacetylene Derivatives: Falcarindiol. Falcarindiol is a common bioactive compound found in some vegetables and medicinal herbs. Falcarindiol $(10 \mu \mathrm{M})$ has been reported to promote ApoA-1-mediated cholesterol efflux in differentiated macrophages by increasing the mRNA and protein expression of ABCA1 via PPAR $\gamma$ (Wang et al., 2017e). One unique mechanism of falcarindiol is that it not only increased ABCA1 gene expression but also prevented cathepsin-dependent ABCA1 protein degradation (Wang et al., 2017e).

5. Marine Natural Products. In addition to the compounds mentioned above, several marine natural products have also demonstrated an antiatherosclerotic potential by inhibiting foam cell formation. For example, spiromastixones 6 and $14(10 \mu \mathrm{M})$ (Wu et al., 2015a) significantly inhibited oxLDL-induced RAW264.7 foam cell formation in mouse macrophages by inhibiting lipid uptake, as well as promoting cholesterol efflux mediated by HDL and ApoA-1. Mechanistic studies reveal that both compounds significantly reduced the expression of CD36, while upregulating PPAR $\gamma / \mathrm{ABCA} 1$ (G1) (Wu et al., 2015a), indicating that both compounds could serve as promising leads with an antiatherogenic potential.

\section{Clinically Used Drugs that Influence Foam Cell Formation}

Macrophage foam cells play a critical role in the development of atherosclerosis (Sharma et al., 2010). The inhibition of foam cell formation is emerging as an attractive strategy for therapeutic intervention of atherosclerosis in clinics.

\section{A. Approved Drugs}

1. Statins. Statins, used as cholesterol-lowering drugs, effectively reduce CVD and mortality in patients with high risk of CVD (Robson, 2008). The mechanism of their action is related to decrease in cholesterol biosynthesis mediated through competitive inhibition of HMG-CoA reductase and their cholesterol-independent pleiotropic effects. Statins are highly efficacious (in lowering atherogenic LDL), safe, and generally well tolerated in patients with CVD. Statins are reported to reduce the risk for developing nonfatal myocardial infarction, ischemic stroke, cardiovascular, and allcause mortality. Statins have also been reported to promote the stabilization and regression of preestablished atherosclerotic plaques (Toth and Banach, 2019). Clinically used statins can be grouped in two categories, the lipophilic statins (such as simvastatin and atorvastatin) and hydrophilic statins (such as fluvastatin, pravastatin, and rosuvastatin). Although statins share a common mechanism of action by inhibiting HMG-CoA reductase, they differ in terms of chemical structures/water solubility, pharmacokinetic properties (absorption, distribution, metabolism, and excretion), and efficacy of modulating lipids (Schachter, 2005). The lipophilicity/hydrophilicity of statins may have differential effects and mechanisms in suppressing endothelial dysfunction, foam cell formation, and CVD development. For example, a systematic metaanalysis showed that hydrophilic statins, but not hydrophobic statins, significantly reduced the level of asymmetric dimethylarginine (which inhibits NO formation and promotes endothelial dysfunction) (Serban et al., 2015). Another meta-analysis showed that both the hydrophilic and the lipophilic statins showed similar risk reduction for major adverse cardiac events, cardiovascular death, all-cause mortality, and statinassociated muscle symptoms. However, the authors observed that hospitalization caused by CVDs was lower and elevation of alanine aminotransferase was higher in lipophilic statin- than in hydrophilic statintreated patients (Bytyçi et al., 2017). In a separate study, Kim et al. (2011) observed that treatment of patients with myocardial infarction with lipophilic statins lead to a better short-term cardiovascular outcome; however, both lipophilic and hydrophilic statins lead to a similar reduction of lipids and 1-year cardiovascular outcomes. Therefore, we might expect different clinical outcomes in different patient populations after 
treatment with lipophilic or hydrophilic statins for different periods.

In cultured macrophages, both lipophilic and hydrophilic statins can ameliorate foam cell formation. For example, rosuvastatin reduces oxLDL-induced foam cell formation in macrophages by reducing CD36 expression, without affecting SR-A expression (Yu et al., 2018). Another study demonstrated that preincubation of THP-1 macrophages with atorvastatin enhanced cholesterol efflux to ApoA-1 and HDL3 dose dependently (Argmann et al., 2005). Atorvastatin also increased PPAR $\gamma$ activity, enhanced LXR activation, and increased ABCA1 expression (Argmann et al., 2005). A recent study showed that reduced protein expression of calpain-1 is accountable for simvastatin-mediated protective effects against foam cell formation (Yang et al., 2016). However, there are several other studies that indicated that simvastatin decreased the ABCA1 protein levels in THP-1 via miRNA-33 (Horie et al., 2010; Niesor et al., 2015). Therefore, overall the effects of statins on macrophage foam cell formation are still controversial. A genome-wide comparison of the differentially expressed genes in macrophage foam cells treated with hydrophilic or lipophilic statins will provide a clear picture of the cardiovascular protective transcriptome of different statins.

2. Nicotinic Acid. Nicotinic acid (NA) has been used for decades as a drug to reduce the progression of atherosclerosis by decreasing LDL-C and increasing HDL-C levels in plasma (Meyers et al., 2004; Carlson, 2005). However, one recent trial suggests that there were no additional clinical effects upon adding niacin to statin therapy during a 36-month follow up period, though HDL-C and TG levels were significantly increased in the patients with atherosclerotic CVDs (Boden et al., 2011). In macrophages from wild-type mice, NA enhanced ABCG1 expression and promoted cholesterol efflux (Lukasova et al., 2011). It is reported that NA increased the production rate of ApoA-1 in both liver and intestinal cells (Rubic et al., 2004; LamonFava et al., 2008), probably through an activation of MAPK and PPAR pathways (Lamon-Fava and Micherone, 2004; Pandey et al., 2008). On the contrary, other studies reported that there are no effects of NA on ApoA-1 production rate (Jin et al., 1997). Furthermore, NA did not exhibit effects on either cholesterol efflux or key RCT gene transcription in THP-1-derived foam cells (Chai et al., 2013).

3. Ezetimibe. Ezetimibe, a selective inhibitor of intestinal cholesterol absorption, has been shown to reduce plasma cholesterol levels and exhibit an antiatherosclerotic effect (Al-Shaer et al., 2004). One study showed that ezetimibe inhibited foam cell formation via the caveolin-1/MAPK signaling pathway, which might be another mechanism of its anti-atherosclerotic effect (Qin et al., 2016).
4. Proprotein Convertase Subtilisin/Kexin Type 9Antibodies. PCSK9 is an enzyme encoded by the PCSK9 gene on chromosome 1 in human beings (Seidah et al., 2003). PCSK9 reduced the amount of LDLRs in hepatocytes by promoting their degradation (Denis et al., 2012). Blocking PCSK9 expression/activity can increase the LDLR amount on the cellular surface and decrease blood LDL-particle concentrations (Weinreich and Frishman, 2014; Joseph and Robinson, 2015). Monoclonal PCSK9 antibodies have been developed as a very effective approach to inhibit PCSK9 and reduce LDL levels (Chaudhary et al., 2017). Several monoclonal antibodies have been or are being tested in clinical studies. Among them, Alirocumab and Evolocumab were recently approved by the Food and Drug Administration for adult patients with heterozygous familial hypercholesterolemia or with clinically significant atherosclerotic CVD requiring additional LDL lowering (Chaudhary et al., 2017). One study displayed that PCSK9 directly decreased cholesterol efflux by the downregulation of ABCA1 expression (Adorni et al., 2017), which might be another mechanism of the antiCVD effect conferred by inhibition of PCSK9.

\section{B. Drugs in Clinical Trials}

1. Glucagon-like Peptide-1 Receptor Agonists. GLP-1 is a gut hormone that activates a G proteincoupled receptor (GLP-1R) in a glucose-dependent manner to stimulate pancreatic $\beta$ cells and secretion of insulin postprandially (Campbell and Drucker, 2013). GLP-1R agonists (e.g., liraglutide, lixisenatide, albiglutide) or incretin mimetics are used as medicines for the treatment of type 2 diabetes. Recent evidence has suggested that the augmentation of GLP-1R signaling by administration of GLP-1R agonists has beneficial effects on the cardiovascular system in patients with diabetes (Ussher and Drucker, 2012). Further study indicated that GLP-1R signaling induced autophagy, thereby suppressing foam cell formation in nonobese subjects. In obese patients, stimulation of GLP-1R promoted formation of foam cell and production of TNF- $\alpha$, IL-1 $\beta$, and IL-6 (Tanaka et al., 2016).

2. High-density Lipoprotein / Apolipoprotein A-1-raising Agents. Current treatment of atherosclerotic CVD is dominated by lowering LDL-C, while increased plasma levels of cholesterol efflux acceptors HDL-C and ApoA-1 expression have been viewed as an alternative therapeutic strategy against CVD for more than three decades (Bailey et al., 2010). The idea to treat CVD by raising HDL comes from a concept relying on the inverse correlation of CVD and human plasma HDL-C (Brewer, 2004), which retrieves excess cholesterol from peripheral cells, including vessel wall macrophages, to the liver for excretion into the bile (Duffy and Rader, 2006). Although there are some animal studies and human trials demonstrating that infusion of HDL or ApoA-1 limit the progression of atherosclerosis (Tall, 
1990; Nissen et al., 2003), a randomized, double-blind study involving 15,067 patients at high cardiovascular risk indicated that the CETP inhibitor torcetrapib raised the levels of HDL particles but failed to counteract atherosclerosis (Barter et al., 2007). Two trials have also compared niacin with placebo, added to simvastatin, and although niacin raised HDL-C by $\sim 15 \%$, there was no clinical benefit to atherosclerosis (Boden et al., 2011; HPS2-THRIVE Collaborative, 2013). These studies indicate the complexity of HDL biology and suggest that not only HDL-C levels but also size, composition, and functionality of the HDL particles may be important. The focus of drug development should shift from raising HDL-C levels to enhancing HDL function. Current knowledge on the role of HDL in preventing various diseases is threefold: 1) HDL itself is not a reliable biomarker/predictor of CVD and/or cancer; 2) the quality/functionality (such as cholesterol efflux assay in patients derived macrophages), rather than the quantity of HDL, is more important for atheroprotection and CVD prevention; and 3) the functionality of HDL plays an important role not only in CVD (such as atherosclerosis), but also in cancer diagnosis/monitoring (Otocka-Kmiecik et al., 2012; Toth et al., 2014; Ganjali et al., 2019; Penson et al., 2019).

RVX-208 (apabetalone) is a selective antagonist of bromodomain and extra terminal (BET) bromodomains (McLure et al., 2013; Picaud et al., 2013). It interferes with the BET protein bromodomain 4 (BRD4), resulting in an increased expression of ApoA-1 in cells, mice, monkeys, and humans (Bailey et al., 2010; Jahagirdar et al., 2014). RVX-208 is being developed as an orally active small molecule for the treatment of CVD (Nicholls et al., 2011). A clinical study showed that RVX-208 increased ApoA-1, pre- $\beta$-HDL, and HDL functionality and increased cholesterol efflux in vitro (Bailey et al., 2010). In two Phase II clinical trials, RVX208 increased the HDL-C and ApoA-1 levels, exhibiting the ability to treat atherosclerosis in patients with established CVD (Johansson et al., 2014). An international, multicenter Phase III trial for treatment of atherosclerosis commenced in October 2015. In 2018, Resverlogix Corp. announced that it has successfully surpassed the planned enrollment target of over 2400 patients in the ongoing Phase III trial (BETonMACE). The scientific community is looking forward to the results of the Phase III trial. The findings discussed here indicate that the regulation of ApoA-1 expression would be a very promising approach for treating atherosclerosis.

One potential therapeutic strategy related to ApoA-1 is the application of small peptides that mimic the ApoA-1 function. ApoA-1 comprises a total of 243 amino acids and involves 10 amphipathic $\alpha$-helices, which are very important for its interaction with lipids. Peptides that mimic the amphipathic helices in ApoA-1 (ApoA-1 mimetic peptides) are lately used as therapeutic agents (such as 4F, 6F, FX-5A, ATI-5261, ETC-642) (Stoekenbroek et al., 2015). At present, these peptides are being tested in preclinical models, which show that they increase macrophage cholesterol efflux and exhibit antiatherosclerosis action (Stoekenbroek et al., 2015). A small pilot study (ApoA-1 Milano clinical trial) indicated that a complex of recombinant ApoA-1 Milano with phospholipid carriers (ETC-216) significantly reduced percentage and total atheroma volume and plaque thickness in patients (Nissen et al., 2003). Pfizer purchased the producer of ETC-216, Esperion Therapeutics in 2003, in the hope of developing a more effective treatment than ETC-216. However, Pfizer did announce a progress with the development of such product. Currently, no drugs based on ApoA-1 Milano are commercially available.

CSL-111, an rHDL, is composed of human ApoA-1 and soybean phosphatidylcholine, which mimics native HDL (Stoekenbroek et al., 2015). In the "Effect of rHDL on Atherosclerosis-Safety and Efficacy" (ERASE) trial, although CSL-111 remarkably decreased atheroma volume compared with baseline, it induced a high incidence of liver function abnormalities (Tardif et al., 2007). Therefore, further clinical study on CSL-111 was not continued. CSL-112, a second-generation of CSL-111 with no liver toxicity, increased the ApoA-1, pre- $\beta$ HDL levels, and ABCA1-mediated cholesterol efflux in two Phase I studies (Krause and Remaley, 2013; Easton et al., 2014). In the Phase II trial, infusion of CSL112 for 4 weeks was well tolerated and did not induce any significant side effects in liver or kidney function. CSL112 significantly enhanced cholesterol efflux (Michael Gibson et al., 2016). In 2018, CSL Behring has started the "ApoA-1 Event reducinG in Ischemic Syndromes II" (AEGIS-II) Phase III clinical trial of CSL112, which is a multicenter, double-blind, randomized, placebo-controlled, parallel-group study. The scientific community is looking forward to the results of the Phase III trial of CSL112.

CER-001, a pre- $\beta$ HDL mimetic, comprises recombinant ApoA-1, diphosphatidylglycerol, and sphingomyelin (Stoekenbroek et al., 2015). In a Phase I trial, infusion of CER-001 was well tolerated and did not show any side effects at any doses (Keyserling et al., 2011). The Phase II trials indicated that CER-001 increased RCT and might significantly decrease aortic atheroma volume. CER-001 has already reached Phase III for the treatment of patients with genetic HDL deficiencies. However, CER-001 has failed in Phase II trial for the treatment of patients with coronary atherosclerosis following acute coronary syndromes (Nicholls et al., 2018). The results show that CER-001 did not produce plaque regression in statin-treated patients following acute coronary syndrome (Nicholls et al., 2018).

3. Bempedoic Acid (ETC-1002). Bempedoic acid (ETC-1002), a small-molecule inhibitor of ATP citrate lyase, has been shown to reduce the level of low-density lipoprotein (LDL) cholesterol and the development of 
atherosclerosis in hypercholesterolemic $\mathrm{ApoE}^{-/-}$mice (Pinkosky et al., 2016), $\mathrm{LDLR}^{-1-}$ mice (Samsoondar et al., 2017), LDLR-deficient miniature pigs (Samsoondar et al., 2017), and statin-intolerant hypercholesterolemic patients (Ballantyne et al., 2018; Laufs et al., 2019). Bempedoic acid, as a prodrug, is converted to the active agent in liver, but not in skeletal muscle; therefore, bempedoic acid may avoid myotoxicity associated with statin therapy (Penson et al., 2017; Ruscica et al., 2019). A recent Phase III clinical trial has shown that treatment with bempedoic acid for 52-weeks added to the maximally tolerated statin therapy significantly lower LDL-C levels without causing higher incidence of overall adverse events (Ray et al., 2019). To date, no information is available whether bempedoic acid can affect foam cell formation. Based on the promising LDLlowering effects of bempedoic acid, it is plausible that bempedoic acid could also affect lipid uptake and cholesterol efflux.

\section{The Role of Inhibition of Foam Cell Formation in Antiatherosclerosis Therapy}

Atherosclerotic lesions in the early stages of atherosclerosis are characterized by the subendothelial accumulation of lipid-laden macrophages (foam cells) in the large arteries (Lusis, 2000). With time, the foam cells eventual demise within the atherosclerotic lesion contributes to their lipid-filled contents in the necrotic core of the advanced lesion, as shown in animal models (Cookson, 1971; Tamminen et al., 1999). In humans, such fatty streak lesions were also found in the aorta, the coronary arteries, and the cerebral arteries (Lusis, 2000), suggesting that foam cell formation also play a critical role in atherosclerotic development in humans. Thus, foam cell formation could be a promising therapeutic target for atherosclerosis.

As we discussed in section II above, disruption of macrophage cholesterol uptake, prevention of CE formation, and enhancement of macrophage cholesterol efflux in animals generally reduced foam cell formation and atherosclerotic plaque area. Although animal models are widely used to study the molecular mechanisms that connect altered cholesterol metabolism to the atherosclerotic plaque progression, the possible differences in atherosclerosis development between animal models and humans must be taken into consideration (Maguire et al., 2019). Therefore, it is very important to ascertain whether inhibition of foam cell formation also contributes to atherosclerosis regression in humans.

As we discussed in section V.A, current clinically used drugs (e.g., statins, NA, ezetimibe, Alirocumab, and Evolocumab) to treat CVDs also exhibit inhibitory effects on macrophage foam cells in vitro and in animal models (Ference et al., 2017). However, it remains to be verified how much their therapeutic effects are attributed to their inhibition on foam cell formation in humans. One clinical study showed that pravastatin has the capacity to reduce lipid content and foam cell accumulation in carotid atherosclerotic lesions (Crisby et al., 2001), which indicates that inhibition of foam cell formation might contribute to its therapeutic effects. NA was demonstrated to significantly increase HDL-C in patients (Lee et al., 2009a), which is an acceptor to mediate cholesterol efflux from macrophages, suggesting that NA might possibly also exhibit its effects by influencing foam cell formation. Intriguingly, treatment with PCSK9 monoclonal antibodies in patients with familial hypercholesterolemia reduced intracellular lipid accumulation in circulating monocytes (Bernelot Moens et al., 2017). Furthermore, this study indicated that LDL-C lowering by inhibition of PCSK9 was paralleled by decreased intracellular lipid accumulation, indicating that LDL-C lowering itself is associated with inhibited foam cell formation (Bernelot Moens et al., 2017). It also suggests that inhibition of PCSK9 reduces monocyte levels of intracellular lipids, and might thereby prevent progression and enhance regression of atherosclerosis (Wu and Ballantyne, 2017). Although these drugs exhibit inhibitory effects on foam cell formation in humans, it is unclear how much the inhibition of foam cell formation contributes to their efficacy on CVDs, since their effect on CVDs is mainly due to pleiotropic activities, such as decrease of cholesterol and LDL-C, antioxidant activity and others.

Also, as we discussed in section V.B, enhancement of HDL-C and ApoA-1 levels has been viewed as alternative therapeutic strategies against CVD. Although HDL mediates a number of atheroprotective processes, such as antioxidative, anti-inflammatory, vasodilatory activities, protection against endothelial cell activation and apoptosis and others; very importantly it is also the critical acceptor to mediate cholesterol efflux from foam cells to inhibit foam cell formation (Linton et al., 2000). Recent clinical reports indicated that HDL cholesterol efflux capacity from macrophages has a strong inverse association with both the likelihood of angiographic $\mathrm{CAD}$ and carotid intima-media thickness, independent on the HDL-C levels (Khera et al., 2011). HDL cholesterol efflux capacity was also inversely related with the incidence of cardiovascular events in a large and multiethnic population cohort (Rohatgi et al., 2014) and with incident coronary heart disease events (Saleheen et al., 2015). These clinical studies indicated that enhancement of cholesterol efflux might decrease CVDs, providing compelling evidence that the inhibition of foam cell formation is antiatherogenic in humans. Moreover, RVX-208 increased the expression of cholesterol efflux acceptor ApoA-1 in humans, reducing major adverse cardiovascular events in treated patients in several Phase II trials as discussed above (section V.B), suggesting targeting foam cell formation might be a promising target to treat CVDs. Unfortunately, these clinical studies were not able to test the 
foam cell formation and accumulation. Therefore, there is no direct and clear evidence to verify how much the inhibition of foam cell formation contributes to the respective therapeutic effects on CVDs. At present, the examined endpoints in these clinical studies include death from $\mathrm{CAD}$, ischemic stroke, nonfatal myocardial infarction, carotid intima-media wall thickness (Cheng et al., 2016), hospitalization for an acute coronary syndrome, symptom-driven coronary or cerebral revascularization (Boden et al., 2011), lipid parameters (e.g., ApoA-1, LDL, HDL) (Rohatgi et al., 2014), none of which can directly and unambiguously represent the form cell formation. In this context, we suggest that future clinical trials may use foam cell formation as a further clinical endpoint to confirm whether inhibition of foam cell formation can directly contribute to anti-CVDs therapy.

\section{Concluding Remarks and Future Perspectives}

Atherosclerosis is traditionally considered as a lipid disorder, in which multiple pathologic factors, such as immunity disorders (Hansson and Hermansson, 2011), unresolved inflammation (Kasikara et al., 2018), and epigenetics (Xu et al., 2018), add to the complexity of the disease. Targeting diverse mechanisms of atherosclerosis could facilitate the therapeutic interventions to combat atherosclerosis (Maguire et al., 2019).

Based on the lipid and inflammation hypothesis of atherosclerosis, lipid-modulating strategies (i.e., by statins, fibrates, and PCSK9 inhibitors) and antiinflammatory therapies (i.e., by IL- $1 \beta$ monoclonal antibody injection) remain the mainstream therapy for atherosclerosis. Macrophages are one main cell type that handles inflammatory response, immunity, and lipid metabolism in the atherosclerotic lesions. Our understanding of how macrophages govern the inflammatory response, lipid transport, and storage within lesions has significantly progressed over the last 10 years. In humans, atherosclerotic lesions (fatty streaks), characterized by the subendothelial accumulation of lipid-laden macrophages (foam cells), were found in the aorta, the coronary arteries, and the cerebral arteries (Lusis, 2000), which were also confirmed in animal models (Cookson, 1971; Tamminen et al., 1999). It was assumed that foam cell formation plays a critical role in atherosclerotic development in humans, and inhibition of foam cell formation would be a promising target to treat atherosclerosis. The formation of foam cell is a multistep cellular process, which involves lipid uptake, cholesterol ester accumulation, and cholesterol efflux. These three processes seem to be independent of each other, but indeed constitute an interrelated gene regulation network. The SR-mediated uptake is the major pathway of cholesterol uptake. CD36, SR-A, and LOX-1 are the major SRs responsible for the binding and following uptake of modified LDL by macrophages (Kunjathoor et al., 2002), whose regulation and functions are addressed in the review. Cholesterol esterification and CE hydrolysis involved in foam cell formation could also be alternative targets for the treatment of atherosclerosis. ACAT1 and ACAT2 involving in cholesterol esterification, as well as NCEH1, LIPA, and lipase E involving in CE hydrolysis are also concluded here. In addition, current understanding of the function and regulation of the cholesterol transporters (ABCA1 and ABCG1), SR-BI, and the acceptors (ApoA-1, HDL and ApoE), which are related to cholesterol efflux from macrophages, are reviewed. Inhibition of LDL uptake, prevention of cholesterol esterification, and promotion of CE hydrolysis, as well as increase of cholesterol efflux in macrophages could represent novel therapeutic modalities of atherosclerosis. A multifaceted approach, which blocks lipid uptake and $\mathrm{CE}$ accumulation while promoting cholesterol efflux, could be the most effective way to reduce foam cell formation with reduced side effects (such as FC accumulation) (Maguire et al., 2019).

There are a variety of models to study the process of foam cell formation and to screen the promising compounds targeting foam cell formation. We reviewed the cellular models for studying cholesterol uptake, cholesterol efflux, and animal models for study on macrophage RCT and foam cell formation. All these models provide a good platform to evaluate the effect of natural products on foam cell formation in vitro and in vivo and to further identify promising drug candidates for treatment of atherosclerosis (Getz and Reardon, 2012; Hilgendorf and Swirski, 2012). However, it should be pointed out that studies in animal models have limited suitability because of significant species differences compared with humans (Lusis, 2000). For example, contradictory results related to the pathologic roles of CD36, SR-A, and ACAT1 in atherosclerosis have indicated the translation of the findings from mice to human as a big challenge for cardiovascular biology and drug discovery. Thus, it is critical to select a unique animal model(s) that mimic human atherosclerosis pathophysiology and to minimize the variables arising from different sexes, study duration, and type of atherogenic diet. Differences in lipid profile, topography of arterial lesions, and the susceptibility to plaque rupture are key factors that must be taken into consideration when choosing the suitable animal model (Emini Veseli et al., 2017).

Natural products remain a promising source for new lead structures. Their great structural diversity and biodiversity makes them an attractive pool in the quest for new therapeutics. In this review, natural products regulating foam cell formation in different models are outlined, including flavonoids, terpenoids, phenolic compounds, phenylpropanoids, alkaloids, steroids, fatty acids, amino acids, carbohydrates, and others. Among them, cyanidin-3-glucoside, ICA, iristectorigenin B, pratensein, 
isosilybin A, $\beta$-carotene, lycopene, erythrodiol, ginsenoside Rb1, tanshindiol C, PGG, berberine, 13-HODE, and spiromastixones appeared to be very active in cellular models. Thus, in vivo corroboration of their activity appears worthwhile. For some of the natural compounds, like anthocyanins, baicalin, chrysin, cyanidin3-O- $\beta$-glucoside, daidzein, ellagic acid, hesperetin, quercetin, isosilybin A, ATRA, 9-cis- $\beta$ c, 9-cis-RA, UA, ginsenoside $\mathrm{Rd}$, zerumbone, curcumin, danshensu, paeonol, protocatechuic acid, salvianolic acid, sesamol derivative (INV-403), resveratrol, EGCG, sesamin, chlorogenic acid, caffeic acid, ferulic acid, rutaecarpine, leonurine, diosgenin, DHA, linoleic acid, allicin, asperlin, and emodin, in vivo studies in models for atherosclerosis already exist, which makes these compounds even more promising. The therapeutic effects of these compounds on foam cell formation and atherosclerosis remain to be investigated in clinical studies. Some natural compounds, like pratensein, astaxanthin, betulinic acid, tanshinone IIA, vitamin D, have even been investigated in clinical trials. Large and multiethnic population cohorts are required to further confirm their therapeutic effect on atherosclerosis.

We also reviewed the effect of clinically used drugs on foam cell formation, including statins, NA, ezetimibe, and PCSK9 antibodies, as well as drugs in clinical trials, such as GLP-1 receptor agonists and HDL/ApoA-1raising agents. Although these drugs exhibit positive effects on foam cell formation in humans, there is no direct evidence to verify how much the inhibition of foam cell formation contributes to the therapeutic effects of these drugs on CVDs.

Future directions in studying the mechanisms of foam cell formation and the discovery of foam celltargeted therapies include:

- Further understanding the regulatory mechanisms of foam cell formation by new biotechnologies (such as RNA-sequencing, lncRNA arrays, RNA interference (RNAi), or CRISPR/Cas9 library screening). Although significant progress has been made recently in characterizing regulatory mechanisms controlling the formation of foam cells, targeting some of these known regulatory mechanisms has yielded negative or controversial results, such as pathologic roles of CD36, SR-A, ACAT1, ABCA1, and rHDL in atherosclerosis. Therefore, it is important to further understand the regulatory mechanisms of foam cell formation by new biotechnologies. The widespread use of RNA-sequencing and lncRNA arrays (Xu, 2017) has empowered the discovery of new epigenetic regulators (such as lncRNAs) that are associated with or regulate foam cell formation, atherosclerosis, and related vascular diseases. Further elucidation of the role of these new regulators will provide us a clear picture of how foam cell formation is regulated and can be therapeutically targeted. Methods of systems biology, such as RNAi or CRISPR/Cas9 library screening are very useful high-throughput methods to elucidate novel regulators of foam cell formation at the genome-wide scale (Domschke et al., 2018). The establishment of new screening systems based on RNAi or CRISPR/Cas9 library screening will significantly accelerate understanding the regulatory mechanisms of foam cell formation.

- High-throughput screening of natural products targeting foam cell formation. Natural products are a well-established source for the development of new drugs in general (Atanasov et al., 2015; Waltenberger et al., 2016). They display a great structural diversity and also cover a large range of biologic activity compared with combinatorial and synthetic compounds (Hiebl et al., 2018). A huge number of natural products were found to regulate ABCA1 expression. Nevertheless, many natural products have never been investigated concerning a potential regulation of foam cell formation. To identify potential drug leads with capability of suppressing foam cell formation from such large pool, high-throughput drug screening platforms are important. For example, a recent study has used a high-throughput screening platform to identify novel candidate genes involved in lipid uptake by human macrophages (Domschke et al., 2018). This platform can similarly be used to identify natural products that have inhibitory effects on lipid uptake.

- Validation of anti-foam cell formation and antiatherosclerotic effect of bioactive natural products in vitro, in vivo, and in clinical studies. Most of studies evaluating the anti-foam cell formation capabilities of natural products were performed using RAW264.7 macrophages and differentiated THP-1 macrophages, two common cell lines used in macrophage biology research. To further evaluate the potential of promising natural products, pharmacological activities need to be validated in primarily cultured HMDMs and, most importantly, in animal models of atherosclerosis. However, we have to bear in mind that the mechanisms of actions of these natural products with potential anti-foam cell formation effects are very wide, ranging from reduction of proinflammatory factors/biomarkers (such as CRP), inhibition of oxidative stress, and direct inhibition of lipid synthesis (by inhibiting the activity of HMG-CoA reductase, PCSK9, or SREBPs). Those natural products may be working in a similar ways to some nutraceuticals (such as polyunsaturated fatty acids) or current lipidmodulating therapies (Sahebkar et al., 2016a,b; 
Serban et al., 2016; Cicero et al., 2017; Momtazi et al., 2017; Ursoniu et al., 2017; Banach et al., 2018; Patti et al., 2018). Another important consideration in future research is to verify whether the anti-foam cell formation effects of natural products directly contribute to their antiatherosclerotic effects, since the progression of atherosclerosis is a complex process. In the light of recent observations that impaired ABCA1-dependent cholesterol efflux associates with markers of inflammasome activation in mouse and human patients with loss-of-function mutations in ABCA1 (Tangier disease) (Westerterp et al., 2018), it is possible that the vicious cycle of "foam cell formation-inflammation/ inflammasome-foam cell formation-atherosclerosis" could perpetuate the pathogenesis of atherosclerosis. Some of the natural compounds exhibit very strong inhibitory effects on foam cell formation in vitro, in vivo, and in small clinical trials. It is very valuable to conduct large and multi-ethnic population cohorts to confirm their therapeutic effect on atherosclerosis.

- Improvement of druggability of promising natural products. Some of the natural products with cardiovascular potential have the disadvantage of low oral bioavailability and low efficacy and have systemic and complex pharmacological effects in vivo. Further structural modification of these promising compounds can lead to antiatherosclerotic drug candidates with higher efficacy and less toxicity/nonspecific effects. In addition, the use of nanotechnology, for example, nanoencapsulation can significantly enhance the stability and antiatherogenic efficacy of natural products, as shown for EGCG in macrophages (Zhang et al., 2013, 2016a; Hong et al., 2014). In the future, it is feasible to design nanomedicines by coupling natural products with macrophage specific marker proteins to achieve targeted delivery of drugs to foam cells in atherosclerotic plaques in vivo.

\section{Authorship Contributions}

Wrote or contributed to the writing of the manuscript: Wang, Yang, Lei, Tzvetkov, Liu, Yeung, Xu, Atanasov.

\section{References}

Abe J and Berk BC (2014) Novel mechanisms of endothelial mechanotransduction. Arterioscler Thromb Vasc Biol 34:2378-2386.

Abramovitz D, Gavri S, Harats D, Levkovitz H, Mirelman D, Miron T, Eilat-Adar S, Rabinkov A, Wilchek M, Eldar M, et al. (1999) Allicin-induced decrease in formation of fatty streaks (atherosclerosis) in mice fed a cholesterol-rich diet. Coron Artery Dis 10:515-519.

Acton S, Rigotti A, Landschulz KT, Xu S, Hobbs HH, and Krieger M (1996) Identification of scavenger receptor SR-BI as a high density lipoprotein receptor. Science 271:518-520.

Adachi H, Tsujimoto M, Arai H, and Inoue K (1997) Expression cloning of a novel scavenger receptor from human endothelial cells. J Biol Chem 272:31217-31220.

Adlakha YK, Khanna S, Singh R, Singh VP, Agrawal A, and Saini N (2013) Proapoptotic miRNA-128-2 modulates ABCA1, ABCG1 and RXR $\alpha$ expression and cholesterol homeostasis. Cell Death Dis 4:e780.
Adorni MP, Cipollari E, Favari E, Zanotti I, Zimetti F, Corsini A, Ricci C, Bernini F, and Ferri N (2017) Inhibitory effect of PCSK9 on Abca1 protein expression and cholesterol efflux in macrophages. Atherosclerosis 256:1-6.

Adorni MP, Zimetti F, Billheimer JT, Wang N, Rader DJ, Phillips MC, and Rothblat GH (2007) The roles of different pathways in the release of cholesterol from macrophages. J Lipid Res 48:2453-2462.

Ahmad S, Alam K, Hossain MM, Fatima M, Firdaus F, Zafeer MF, Arif Z, Ahmed M, and Nafees KA (2016) Anti-arthritogenic and cardioprotective action of hesperidin and daidzein in collagen-induced rheumatoid arthritis. Mol Cell Biochem 423: $115-127$.

Aizawa K and Inakuma T (2009) Dietary capsanthin, the main carotenoid in paprika (Capsicum annuum), alters plasma high-density lipoprotein-cholesterol levels and hepatic gene expression in rats. Br J Nutr 102:1760-1766.

Aktan F, Henness S, Tran VH, Duke CC, Roufogalis BD, and Ammit AJ (2006) Gingerol metabolite and a synthetic analogue capsarol inhibit macrophage NFkappaB-mediated iNOS gene expression and enzyme activity. Planta Med $\mathbf{7 2}$ : $727-734$

Alam K, Meidell RS, and Spady DK (2001) Effect of up-regulating individual steps in the reverse cholesterol transport pathway on reverse cholesterol transport in normolipidemic mice. J Biol Chem 276:15641-15649.

Aleidi SM, Howe V, Sharpe LJ, Yang A, Rao G, Brown AJ, and Gelissen IC (2015) The E3 ubiquitin ligases, HUWE1 and NEDD4-1, are involved in the post-translational regulation of the ABCG1 and ABCG4 lipid transporters. J Biol Chem 290: 24604-24613.

Alfaidi MA, Chamberlain J, Rothman A, Crossman D, Villa-Uriol MC, Hadoke P, Wu J, Schenkel T, Evans PC, and Francis SE (2018) Dietary docosahexaenoic acid reduces oscillatory wall shear stress, atherosclerosis, and hypertension, most likely mediated via an IL-1-mediated mechanism. J Am Heart Assoc 7:e008757.

Alique M, Luna C, Carracedo J, and Ramírez R (2015) LDL biochemical modifications: a link between atherosclerosis and aging. Food Nutr Res 59:29240.

Al-Jarallah A, Chen X, González L, and Trigatti BL (2014) High density lipoprotein stimulated migration of macrophages depends on the scavenger receptor class B, type I, PDZK1 and Akt1 and is blocked by sphingosine 1 phosphate receptor antagonists. PLoS One 9:e106487.

Allahverdian S, Chehroudi AC, McManus BM, Abraham T, and Francis GA (2014) Contribution of intimal smooth muscle cells to cholesterol accumulation and macrophage-like cells in human atherosclerosis. Circulation 129:1551-1559.

Allahverdian S, Pannu PS, and Francis GA (2012) Contribution of monocyte-derived macrophages and smooth muscle cells to arterial foam cell formation. Cardiovasc Res 95:165-172.

Allen AM and Graham A (2012) Mitochondrial function is involved in regulation of cholesterol efflux to apolipoprotein (apo)A-I from murine RAW 264.7 macrophages. Lipids Health Dis 11:169.

Al-Shaer MH, Choueiri NE, and Suleiman ES (2004) The pivotal role of cholesterol absorption inhibitors in the management of dyslipidemia. Lipids Health Dis 3:22. Ambros V (2004) The functions of animal microRNAs. Nature 431:350-355.

Anandhi R, Thomas PA, and Geraldine P (2014) Evaluation of the anti-atherogenic potential of chrysin in Wistar rats. Mol Cell Biochem 385:103-113.

Angelovich TA, Hearps AC, Maisa A, Kelesidis T, and Jaworowski A (2017) Quantification of monocyte transmigration and foam cell formation from individuals with chronic inflammatory conditions. J Vis Exp 128:56293.

Antunez-Solis J, Hernández-Derramadero F, Aquino-Vega M, Ibarra-Ramírez S, Rodríguez-Páez L, Baeza I, and Wong C (2009) 2,4,5-trimethoxycinnamic acid: the major metabolite of alpha-asarone, retains most of the pharmacological properties of alpha-asarone. J Enzyme Inhib Med Chem 24:903-909.

Arbab-Zadeh A, Nakano M, Virmani R, and Fuster V (2012) Acute coronary events. Circulation 125:1147-1156.

Argmann CA, Edwards JY, Sawyez CG, O’Neil CH, Hegele RA, Pickering JG, and Huff MW (2005) Regulation of macrophage cholesterol efflux through hydroxymethylglutaryl-CoA reductase inhibition: a role for RhoA in ABCA1mediated cholesterol efflux. J Biol Chem 280:22212-22221.

Asztalos BF, de la Llera-Moya M, Dallal GE, Horvath KV, Schaefer EJ, and Rothblat GH (2005) Differential effects of HDL subpopulations on cellular ABCA1- and SR BI-mediated cholesterol efflux. J Lipid Res 46:2246-2253.

Atanasov AG, Waltenberger B, Pferschy-Wenzig EM, Linder T, Wawrosch C, Uhrin P, Temml V, Wang L, Schwaiger S, Heiss EH, et al. (2015) Discovery and resupply of pharmacologically active plant-derived natural products: a review. Biotechnol Adv 33:1582-1614.

Atanasov AG, Wang JN, Gu SP, Bu J, Kramer MP, Baumgartner L, Fakhrudin N, Ladurner A, Malainer C, Vuorinen A, et al. (2013) Honokiol: a non-adipogenic PPAR $\gamma$ agonist from nature. Biochim Biophys Acta 1830:4813-4819.

Atshaves BP, Starodub O, McIntosh A, Petrescu A, Roths JB, Kier AB, and Schroeder F (2000) Sterol carrier protein-2 alters high density lipoprotein-mediated cholesterol efflux. J Biol Chem 275:36852-36861.

Aviram M, Volkova N, Coleman R, Dreher M, Reddy MK, Ferreira D, and Rosenblat M (2008) Pomegranate phenolics from the peels, arils, and flowers are antiatherogenic: studies in vivo in atherosclerotic apolipoprotein e-deficient (E 0$)$ mice and in vitro in cultured macrophages and lipoproteins. J Agric Food Chem 56: 1148-1157.

Ayaori M, Yakushiji E, Ogura M, Nakaya K, Hisada T, Uto-Kondo H, Takiguchi S, Terao Y, Sasaki M, Komatsu T, et al. (2012) Retinoic acid receptor agonists regulate expression of ATP-binding cassette transporter G1 in macrophages. Biochim Biophys Acta 1821:561-572.

Bäck M and Hansson GK (2019) Omega-3 fatty acids, cardiovascular risk, and the resolution of inflammation. FASEB J 33:1536-1539.

Badimon JJ, Badimon L, and Fuster V (1990) Regression of atherosclerotic lesions by high density lipoprotein plasma fraction in the cholesterol-fed rabbit. J Clin Invest 85:1234-1241.

Baeyens N, Bandyopadhyay C, Coon BG, Yun S, and Schwartz MA (2016) Endothelial fluid shear stress sensing in vascular health and disease. J Clin Invest 126: 821-828. 
Baigent C, Keech A, Kearney PM, Blackwell L, Buck G, Pollicino C, Kirby A, Sourjina T, Peto R, Collins R, et al.; Cholesterol Treatment Trialists' (CTT) Collaborators (2005) Efficacy and safety of cholesterol-lowering treatment: prospective metaanalysis of data from 90,056 participants in 14 randomised trials of statins [published correction appears in Lancet (2005) 366:1358; Lancet (2008) 371:2084] Lancet 366:1267-1278.

Bailey D, Jahagirdar R, Gordon A, Hafiane A, Campbell S, Chatur S, Wagner GS, Hansen HC, Chiacchia FS, Johansson J, et al. (2010) RVX-208: a small molecule that increases apolipoprotein A-I and high-density lipoprotein cholesterol in vitro and in vivo. $J$ Am Coll Cardiol 55:2580-2589.

Bak EJ, Kim J, Choi YH, Kim JH, Lee DE, Woo GH, Cha JH, and Yoo YJ (2014) Wogonin ameliorates hyperglycemia and dyslipidemia via PPAR $\alpha$ activation in $\mathrm{db} /$ db mice. Clin Nutr 33:156-163.

Bale PM, Clifton-Bligh P, Benjamin BN, and Whyte HM (1971) Pathology of Tangier disease. J Clin Pathol 24:609-616.

Ballantyne CM, Banach M, Mancini GBJ, Lepor NE, Hanselman JC, Zhao X and Leiter LA (2018) Efficacy and safety of bempedoic acid added to ezetimibe in statin-intolerant patients with hypercholesterolemia: a randomized, placebocontrolled study. Atherosclerosis 277:195-203.

Banach M, Patti AM, Giglio RV, Cicero AFG, Atanasov AG, Bajraktari G, Bruckert E Descamps O, Djuric DM, Ezhov M, et al.; International Lipid Expert Panel (ILEP) (2018) The role of nutraceuticals in statin intolerant patients. J Am Coll Cardiol 72:96-118.

Bao L, Zhang Y, Wei G, Wang Y, Ma R, Cheng R, Ren X, and Agula B (2015) The antiatherosclerotic effects of puerarin on induced-atherosclerosis in rabbits. Biomed Pap Med Fac Univ Palacky Olomouc Czech Repub 159:53-59.

Bao MH, Zhang YW, and Zhou HH (2013) Paeonol suppresses oxidized low-density lipoprotein induced endothelial cell apoptosis via activation of LOX-1/p38MAPK NF-кB pathway. J Ethnopharmacol 146:543-551.

Bao Y, Wang L, Xu Y, Yang Y, Wang L, Si S, Cho S, and Hong B (2012) Salvianolic acid $B$ inhibits macrophage uptake of modified low density lipoprotein (mLDL) in a scavenger receptor CD36-dependent manner. Atherosclerosis 223:152-159.

Barr DP, Russ EM, and Eder HA (1951) Protein-lipid relationships in human plasma. II. In atherosclerosis and related conditions. Am J Med 11:480-493.

Barros J, Serrani-Yarce JC, Chen F, Baxter D, Venables BJ, and Dixon RA (2016) Role of bifunctional ammonia-lyase in grass cell wall biosynthesis. Nat Plants 2:16050.

Bartel DP (2009) MicroRNAs: target recognition and regulatory functions. Cell 136 215-233.

Barter PJ, Caulfield M, Eriksson M, Grundy SM, Kastelein JJ, Komajda M, LopezSendon J, Mosca L, Tardif JC, Waters DD, et al.; ILLUMINATE Investigators (2007) Effects of torcetrapib in patients at high risk for coronary events. $N$ Engl J Med 357:2109-2122.

Bashtovyy D, Jones MK, Anantharamaiah GM, and Segrest JP (2011) Sequence conservation of apolipoprotein A-I affords novel insights into HDL structurefunction. J Lipid Res 52:435-450.

Beaven SW and Tontonoz P (2006) Nuclear receptors in lipid metabolism: targeting the heart of dyslipidemia. Annu Rev Med 57:313-329.

Bechor S, Zolberg Relevy N, Harari A, Almog T, Kamari Y, Ben-Amotz A, Harats D, and Shaish A (2016) 9-cis $\beta$-carotene increased cholesterol efflux to HDL in macrophages. Nutrients 8:E435.

Bell TA III, Brown JM, Graham MJ, Lemonidis KM, Crooke RM, and Rudel LL (2006) Liver-specific inhibition of acyl-coenzyme a:cholesterol acyltransferase 2 with antisense oligonucleotides limits atherosclerosis development in apolipoprotein B100-only low-density lipoprotein receptor-/- mice. Arterioscler Thromb Vasc Biol 26:1814-1820.

Bell TA III, Kelley K, Wilson MD, Sawyer JK, and Rudel LL (2007) Dietary fatinduced alterations in atherosclerosis are abolished by ACAT2-deficiency in ApoB100 only, LDLr-/- mice. Arterioscler Thromb Vasc Biol 27:1396-1402.

Ben J, Zhu X, Zhang H, and Chen Q (2015) Class A1 scavenger receptors in cardiovascular diseases. Br J Pharmacol 172:5523-5530.

Bennett MR, Sinha S, and Owens GK (2016) Vascular smooth muscle cells in atherosclerosis. Circ Res 118:692-702.

Bensinger SJ, Bradley MN, Joseph SB, Zelcer N, Janssen EM, Hausner MA, Shih R, Parks JS, Edwards PA, Jamieson BD, et al. (2008) LXR signaling couples sterol metabolism to proliferation in the acquired immune response. Cell 134:97-111.

Bentzon JF, Skovenborg E, Hansen C, Møller J, de Gaulejac NS, Proch J, and Falk E (2001) Red wine does not reduce mature atherosclerosis in apolipoprotein E-deficient mice. Circulation 103:1681-1687.

Berbée JF, Wong MC, Wang Y, van der Hoorn JW, Khedoe PP, van Klinken JB, Mol IM, Hiemstra PS, Tsikas D, Romijn JA, et al. (2013) Resveratrol protects agains atherosclerosis, but does not add to the antiatherogenic effect of atorvastatin, in APOE*3-Leiden.CETP mice. J Nutr Biochem 24:1423-1430.

Bernelot Moens SJ, Neele AE, Kroon J, van der Valk FM, Van den Bossche J, Hoeksema MA, Hoogeveen RM, Schnitzler JG, Baccara-Dinet MT, Manvelian G, et al. (2017) PCSK9 monoclonal antibodies reverse the pro-inflammatory profile of monocytes in familial hypercholesterolaemia. Eur Heart $J$ 38:1584-1593.

Berrougui H, Grenier G, Loued S, Drouin G, and Khalil A (2009) A new insight into resveratrol as an atheroprotective compound: inhibition of lipid peroxidation and enhancement of cholesterol efflux. Atherosclerosis 207:420-427.

Besler C, Heinrich K, Rohrer L, Doerries C, Riwanto M, Shih DM, Chroni A, Yonekawa K, Stein S, Schaefer N, et al. (2011) Mechanisms underlying adverse effects of HDL on eNOS-activating pathways in patients with coronary artery disease. $J$ Clin Invest 121:2693-2708.

Besler C, Lüscher TF, and Landmesser U (2012) Molecular mechanisms of vascular effects of high-density lipoprotein: alterations in cardiovascular disease. $E M B O$ Mol Med 4:251-268.

Bess E, Fisslthaler B, Frömel T, and Fleming I (2011) Nitric oxide-induced activation of the AMP-activated protein kinase $\alpha 2$ subunit attenuates I $_{\kappa} \mathrm{B}$ kinase activity and inflammatory responses in endothelial cells. PLoS One 6:e20848.

Binesh A, Devaraj SN, and Devaraj H (2018) Inhibition of nuclear translocation of notch intracellular domain (NICD) by diosgenin prevented atherosclerotic disease progression. Biochimie 148:63-71.
Borradaile NM, de Dreu LE, Wilcox LJ, Edwards JY, and Huff MW (2002) Soya phytoestrogens, genistein and daidzein, decrease apolipoprotein B secretion from HepG2 cells through multiple mechanisms. Biochem $J$ 366:531-539.

Boden WE, Probstfield JL, Anderson T, Chaitman BR, Desvignes-Nickens P, Koprowicz K, McBride R, Teo K, and Weintraub W; AIM-HIGH Investigators (2011) Niacin in patients with low HDL cholesterol levels receiving intensive statin therapy [published correction appears in $N$ Engl J Med (2012) 367:189]. N Engl I Med 365:2255-2267.

Bowden KL, Dubland JA, Chan T, Xu YH, Grabowski GA, Du H, and Francis GA (2018) LAL (lysosomal acid lipase) promotes reverse cholesterol transport in vitro and in vivo. Arterioscler Thromb Vasc Biol 38:1191-1201.

Brewer HB Jr (2004) High-density lipoproteins: a new potential therapeutic target for the prevention of cardiovascular disease. Arterioscler Thromb Vasc Biol 24: 387-391.

Brown MS, Goldstein JL, Krieger M, Ho YK, and Anderson RG (1979) Reversible accumulation of cholesteryl esters in macrophages incubated with acetylated lipoproteins. J Cell Biol 82:597-613.

Brundert M, Heeren J, Bahar-Bayansar M, Ewert A, Moore KJ, and Rinninger F (2006) Selective uptake of HDL cholesteryl esters and cholesterol efflux from mouse peritoneal macrophages independent of SR-BI. J Lipid Res 47:2408-2421.

Brunham LR, Kruit JK, Iqbal J, Fievet C, Timmins JM, Pape TD, Coburn BA, Bissada N, Staels B, Groen AK, et al. (2006) Intestinal ABCA1 directly contributes to HDL biogenesis in vivo. J Clin Invest 116:1052-1062.

Buchebner M, Pfeifer T, Rathke N, Chandak PG, Lass A, Schreiber R, Kratzer A Zimmermann R, Sattler W, Koefeler H, et al. (2010) Cholesteryl ester hydrolase activity is abolished in HSL-/- macrophages but unchanged in macrophages lacking KIAA1363. J Lipid Res 51:2896-2908.

Bumrungpert A, Lilitchan S, Tuntipopipat S, Tirawanchai N, and Komindr S (2018) Ferulic acid supplementation improves lipid profiles, oxidative stress, and inflammatory status in hyperlipidemic subjects: a randomized, double-blind, placebocontrolled clinical trial. Nutrients 10:E713.

Burgess B, Naus K, Chan J, Hirsch-Reinshagen V, Tansley G, Matzke L, Chan B, Wilkinson A, Fan J, Donkin J, et al. (2008) Overexpression of human ABCG1 does not affect atherosclerosis in fat-fed ApoE-deficient mice. Arterioscler Thromb Vasc Biol 28:1731-1737.

Bytyçi I, Bajraktari G, Bhatt DL, Morgan CJ, Ahmed A, Aronow WS, and Banach M; Lipid and Blood Pressure Meta-analysis Collaboration (LBPMC) Group (2017) Hydrophilic vs lipophilic statins in coronary artery disease: a meta-analysis of randomized controlled trials. J Clin Lipidol 11:624-637.

Cameron J, Ranheim T, Kulseth MA, Leren TP, and Berge KE (2008) Berberine decreases PCSK9 expression in HepG2 cells. Atherosclerosis 201:266-273.

Campbell JE and Drucker DJ (2013) Pharmacology, physiology, and mechanisms of incretin hormone action. Cell Metab 17:819-837.

Cao WM, Murao K, Imachi H, Yu X, Dobashi H, Yoshida K, Muraoka T, Kotsuna N, Nagao S, Wong NC, et al. (2004) Insulin-like growth factor-i regulation of hepatic scavenger receptor class BI. Endocrinology 145:5540-5547.

Carlson LA (2005) Nicotinic acid: the broad-spectrum lipid drug. A 50th anniversary review. J Intern Med 258:94-114.

Cases S, Novak S, Zheng YW, Myers HM, Lear SR, Sande E, Welch CB, Lusis AJ, Spencer TA, Krause BR, et al. (1998) ACAT-2, a second mammalian acyl-CoA cholesterol acyltransferase. Its cloning, expression, and characterization. $J$ Biol Chem 273:26755-26764.

Cazarolli LH, Zanatta L, Alberton EH, Figueiredo MS, Folador P, Damazio RG, Pizzolatti MG, and Silva FR (2008) Flavonoids: prospective drug candidates. Mini Rev Med Chem 8:1429-1440.

Chai JT, Digby JE, Ruparelia N, Jefferson A, Handa A, and Choudhury RP (2013) Nicotinic acid receptor GPR109A is down-regulated in human macrophage-derived foam cells. PLoS One 8:e62934.

Chang CC, Huh HY, Cadigan KM, and Chang TY (1993) Molecular cloning and functional expression of human acyl-coenzyme A:cholesterol acyltransferase cDNA in mutant Chinese hamster ovary cells. J Biol Chem 268:20747-20755.

Chang GR, Chen PL, Hou PH, and Mao FC (2015) Resveratrol protects against dietinduced atherosclerosis by reducing low-density lipoprotein cholesterol and inhibiting inflammation in apolipoprotein E-deficient mice. Iran J Basic Med Sci 18:1063-1071.

Chang YC, Lee TS, and Chiang AN (2012) Quercetin enhances ABCA1 expression and cholesterol efflux through a p38-dependent pathway in macrophages. J Lipid Res 53:1840-1850.

Chaudhary R, Garg J, Shah N, and Sumner A (2017) PCSK9 inhibitors: a new era of lipid lowering therapy. World J Cardiol 9:76-91.

Chen CY, Shyue SK, Ching LC, Su KH, Wu YL, Kou YR, Chiang AN, Pan CC, and Lee TS (2011) Wogonin promotes cholesterol efflux by increasing protein phosphatase 2B-dependent dephosphorylation at ATP-binding cassette transporter-A1 in macrophages. $J$ Nutr Biochem 22:1015-1021.

Chen DD, Hui LL, Zhang XC, and Chang Q (2018a) NEAT1 contributes to ox-LDLinduced inflammation and oxidative stress in macrophages through inhibiting miR-128. J Cell Biochem DOI: 10.1002/jcb.27541 [published ahead of print]

Chen FX and Wang LK (2015) Effect of ferulic acid on cholesterol efflux in macrophage foam cell formation and potential mechanism. Zhongguo Zhong Yao Za Zhi 40:533-537.

Chen H, Rossier C, Lalioti MD, Lynn A, Chakravarti A, Perrin G, and Antonarakis SE (1996) Cloning of the cDNA for a human homologue of the Drosophila white gene and mapping to chromosome 21q22.3. Am J Hum Genet 59:66-75.

Chen J, Dai M, and Wang Y (2014) Paeonol inhibits proliferation of vascular smooth muscle cells stimulated by high glucose via Ras-Raf-ERK1/2 signaling pathway in coculture model. Evid Based Complement Alternat Med 2014:484269.

Chen JJ, Dai M, and Chen P (2013) Effect of paeonol on LPS-induced rat vascular endothelial cell adhesion reaction. Zhong Yao Cai 36:433-437.

Chen L, Yao Q, Xu S, Wang H, and Qu P (2018b) Inhibition of the NLRP3 inflammasome attenuates foam cell formation of THP-1 macrophages by suppressing oxLDL uptake and promoting cholesterol efflux. Biochem Biophys Res Commun 495 : $382-387$. 
Chen ML, Yi L, Zhang Y, Zhou X, Ran L, Yang J, Zhu JD, Zhang QY, and Mi MT (2016) Resveratrol attenuates trimethylamine-N-oxide (TMAO)-induced atherosclerosis by regulating TMAO synthesis and bile acid metabolism via remodeling of the gut microbiota. MBio 7:e02210-e02215.

Chen Q, Reis SE, Kammerer C, Craig WY, LaPierre SE, Zimmer EL, McNamara DM, Pauly DF, Sharaf B, Holubkov R, et al. (2003) Genetic variation in lectin-like oxidized low-density lipoprotein receptor 1 (LOX1) gene and the risk of coronary artery disease. Circulation 107:3146-3151.

Chen SJ, Kao YH, Jing L, Chuang YP, Wu WL, Liu ST, Huang SM, Lai JH, Ho LJ, Tsai MC, et al. (2017) Epigallocatechin-3-gallate reduces scavenger receptor A expression and foam cell formation in human macrophages. J Agric Food Chem $\mathbf{6 5}$ 3141-3150.

Chen YL, Hu CS, Lin FY, Chen YH, Sheu LM, Ku HH, Shiao MS, Chen JW, and Lin SJ (2006) Salvianolic acid B attenuates cyclooxygenase-2 expression in vitro in LPS-treated human aortic smooth muscle cells and in vivo in the apolipoprotein-Edeficient mouse aorta. J Cell Biochem 98:618-631.

Cheng JM, Oemrawsingh RM, Garcia-Garcia HM, Boersma E, van Geuns RJ, Serruys PW, Kardys I, and Akkerhuis KM (2016) PCSK9 in relation to coronary plaque inflammation: results of the ATHEROREMO-IVUS study. Atherosclerosis 248:117-122.

Cherepanova OA, Gomez D, Shankman LS, Swiatlowska P, Williams J, Sarmento OF, Alencar GF, Hess DL, Bevard MH, Greene ES, et al. (2016) Activation of the pluripotency factor OCT4 in smooth muscle cells is atheroprotective. Nat Med 22: 657-665.

Chi L, Peng L, Hu X, Pan N, and Zhang Y (2014a) Berberine combined with atorvastatin downregulates LOX-1 expression through the ET-1 receptor in monocyte/ macrophages. Int $J$ Mol Med 34:283-290.

Chi L, Peng L, Pan N, Hu X, and Zhang Y (2014b) The anti-atherogenic effects of berberine on foam cell formation are mediated through the upregulation of sirtuin 1. Int J Mol Med 34:1087-1093.

Chiba T, Ikeda M, Umegaki K, and Tomita T (2011) Estrogen-dependent activation of neutral cholesterol ester hydrolase underlying gender difference of atherogenesis in apoE-/- mice. Atherosclerosis 219:545-551.

Chinetti G, Lestavel S, Bocher V, Remaley AT, Neve B, Torra IP, Teissier E, Minnich A, Jaye M, Duverger N, et al. (2001) PPAR-alpha and PPAR-gamma activators induce cholesterol removal from human macrophage foam cells through stimulation of the ABCA1 pathway. Nat Med 7:53-58.

Chistiakov DA, Bobryshev YV, and Orekhov AN (2016a) Macrophage-mediated cholesterol handling in atherosclerosis. J Cell Mol Med 20:17-28.

Chistiakov DA, Orekhov AN, and Bobryshev YV (2016b) ApoA1 and ApoA1-specific self-antibodies in cardiovascular disease. Lab Invest 96:708-718.

Chiu JJ and Chien S (2011) Effects of disturbed flow on vascular endothelium: pathophysiological basis and clinical perspectives. Physiol Rev 91:327-387.

Cho IJ, Ahn JY, Kim S, Choi MS, and Ha TY (2008) Resveratrol attenuates the expression of HMG-CoA reductase mRNA in hamsters. Biochem Biophys Res Commun 367:190-194.

Choi JS, Choi YJ, Shin SY, Li J, Kang SW, Bae JY, Kim DS, Ji GE, Kang JS, and Kang YH (2008) Dietary flavonoids differentially reduce oxidized LDL-induced apoptosis in human endothelial cells: role of MAPK- and JAK/STAT-signaling. J Nutr 138:983-990.

Choi KW, Park HJ, Jung DH, Kim TW, Park YM, Kim BO, Sohn EH, Moon EY, Um $\mathrm{SH}$, Rhee DK, et al. (2010) Inhibition of TNF- $\alpha$-induced adhesion molecule expression by diosgenin in mouse vascular smooth muscle cells via downregulation of the MAPK, Akt and NF-кB signaling pathways. Vascul Pharmacol 53:273-280.

Choy HA, Wang XP, and Schotz MC (2003) Reduced atherosclerosis in hormonesensitive lipase transgenic mice overexpressing cholesterol acceptors. Biochim Biophys Acta 1634:76-85.

Chyu KY, Babbidge SM, Zhao X, Dandillaya R, Rietveld AG, Yano J, Dimayuga P, Cercek B, and Shah PK (2004) Differential effects of green tea-derived catechin on developing versus established atherosclerosis in apolipoprotein E-null mice. Circulation 109:2448-2453.

Cicero AFG, Colletti A, Bajraktari G, Descamps O, Djuric DM, Ezhov M, Fras Z, Katsiki N, Langlois M, Latkovskis G, et al. (2017) Lipid lowering nutraceuticals in clinical practice: position paper from an International Lipid Expert Panel. Arch Med Sci 13:965-1005.

Collot-Teixeira S, Martin J, McDermott-Roe C, Poston R, and McGregor JL (2007) CD36 and macrophages in atherosclerosis. Cardiovasc Res 75:468-477.

Constantinescu E, Alexandru D, Alexandru V, Raicu M, and Simionescu M (2000) Endothelial cell-derived foam cells fail to express adhesion molecules (ICAM-1 and VCAM-1) for monocytes. J Submicrosc Cytol Pathol 32:195-201.

Cookson FB (1971) The origin of foam cells in atherosclerosis. Br J Exp Pathol 52: 62-69.

Costet P, Lalanne F, Gerbod-Giannone MC, Molina JR, Fu X, Lund EG, Gudas LJ, and Tall AR (2003) Retinoic acid receptor-mediated induction of ABCA1 in macrophages. Mol Cell Biol 23:7756-7766.

Covey SD, Krieger M, Wang W, Penman M, and Trigatti BL (2003) Scavenger receptor class B type I-mediated protection against atherosclerosis in LDL receptornegative mice involves its expression in bone marrow-derived cells. Arterioscler Thromb Vasc Biol 23:1589-1594.

Cragg GM and Newman DJ (2013) Natural products: a continuing source of novel drug leads. Biochim Biophys Acta 1830:3670-3695.

Crisby M, Nordin-Fredriksson G, Shah PK, Yano J, Zhu J, and Nilsson J (2001) Pravastatin treatment increases collagen content and decreases lipid content, in flammation, metalloproteinases, and cell death in human carotid plaques: implications for plaque stabilization. Circulation 103:926-933.

Cui Y, Hou P, Li F, Liu Q, Qin S, Zhou G, Xu X, Si Y, and Guo S (2017) Quercetin improves macrophage reverse cholesterol transport in apolipoprotein E-deficient mice fed a high-fat diet. Lipids Health Dis 16:9.

Curtiss LK and Boisvert WA (2000) Apolipoprotein E and atherosclerosis. Curr Opin Lipidol 11:243-251.

Cushnie TP and Lamb AJ (2011) Recent advances in understanding the antibacterial properties of flavonoids. Int J Antimicrob Agents 38:99-107.
Dai M, Liu QY, Gu CG, and Zhang HY (2000) Inhibitory effect of paeonol on lipid peroxidational reaction and oxidational decorate of low density lipoprotein. Zhongguo Zhong Yao Za Zhi 25:625-627.

Dai M, Zhi X, Peng D, and Liu Q (1999) Inhibitory effect of paeonol on experimental atherosclerosis in quails. Zhongguo Zhong Yao Za Zhi 24:488-490.

Dai Y, Condorelli G, and Mehta JL (2016) Scavenger receptors and non-coding RNAs: relevance in atherogenesis. Cardiovasc Res 109:24-33.

Das R, Ganapathy S, Mahabeleshwar GH, Drumm C, Febbraio M, Jain MK, and Plow EF (2013) Macrophage gene expression and foam cell formation are regulated by plasminogen [published correction appears in Circulation (2014) 129: e326]. Circulation 127:1209-1218, e1-16.

Daub K, Langer H, Seizer P, Stellos K, May AE, Goyal P, Bigalke B, Schönberger T, Geisler T, Siegel-Axel D, et al. (2006) Platelets induce differentiation of human CD34+ progenitor cells into foam cells and endothelial cells. FASEB J 20: $2559-2561$.

Daugherty A (2002) Mouse models of atherosclerosis. Am J Med Sci 323:3-10.

Davidson WS and Thompson TB (2007) The structure of apolipoprotein A-I in high density lipoproteins. J Biol Chem 282:22249-22253.

Davies PF (2009) Hemodynamic shear stress and the endothelium in cardiovascular pathophysiology. Nat Clin Pract Cardiovasc Med 6:16-26.

Dean M, Hamon Y, and Chimini G (2001) The human ATP-binding cassette (ABC) transporter superfamily. J Lipid Res 42:1007-1017.

Dekroon RM and Armati PJ (2002) The effects of oxidative stress and altered intracellular calcium levels on vesicular transport of apoE-EGFP. Cell Biol Int 26: $407-420$

de la Llera-Moya M, Drazul-Schrader D, Asztalos BF, Cuchel M, Rader DJ, and Rothblat GH (2010) The ability to promote efflux via ABCA1 determines the capacity of serum specimens with similar high-density lipoprotein cholesterol to remove cholesterol from macrophages. Arterioscler Thromb Vasc Biol 30:796-801.

Del Bo' C, Cao Y, Roursgaard M, Riso P, Porrini M, Loft S, and Møller P (2016) Anthocyanins and phenolic acids from a wild blueberry (Vaccinium angustifolium) powder counteract lipid accumulation in THP-1-derived macrophages. Eur J Nutr 55:171-182.

Delsing DJ, Offerman EH, van Duyvenvoorde W, van Der Boom H, de Wit EC, Gijbels MJ, van Der Laarse A, Jukema JW, Havekes LM, and Princen HM (2001) AcylCoA:cholesterol acyltransferase inhibitor avasimibe reduces atherosclerosis in addition to its cholesterol-lowering effect in ApoE*3-Leiden mice. Circulation 103: 1778-1786.

Deng YH, Alex D, Huang HQ, Wang N, Yu N, Wang YT, Leung GP, and Lee SM (2011) Inhibition of TNF- $\alpha$-mediated endothelial cell-monocyte cell adhesion and adhesion molecules expression by the resveratrol derivative, trans-3,5,4'-trimethoxystilbene. Phytother Res 25:451-457.

Denis M, Bissonnette R, Haidar B, Krimbou L, Bouvier M, and Genest J (2003) Expression, regulation, and activity of ABCA1 in human cell lines. Mol Genet Metab 78:265-274.

Denis M, Marcinkiewicz J, Zaid A, Gauthier D, Poirier S, Lazure C, Seidah NG, and Prat A (2012) Gene inactivation of proprotein convertase subtilisin/kexin type 9 reduces atherosclerosis in mice. Circulation 125:894-901.

de Sousa RR, Queiroz KC, Souza AC, Gurgueira SA, Augusto AC, Miranda MA, Peppelenbosch MP, Ferreira CV, and Aoyama H (2007) Phosphoprotein levels, MAPK activities and NFkappaB expression are affected by fisetin. J Enzyme Inhib Med Chem 22:439-444.

De Winther MP, Gijbels MJ, Van Dijk KW, Havekes LM, and Hofker MH (2000) Transgenic mouse models to study the role of the macrophage scavenger receptor class A in atherosclerosis. Int J Tissue React 22:85-91.

Dias DA, Urban S, and Roessner U (2012) A historical overview of natural products in drug discovery. Metabolites 2:303-336.

Di Pietro M, De Santis F, Schiavoni G, Filardo S, and Sessa R (2013) Resveratrol in Chlamydia pneumoniae-induced foam cell formation and interleukin-17A synthesis. J Biol Regul Homeost Agents 27:509-518.

Dirsch VM, Kiemer AK, Wagner H, and Vollmar AM (1998) Effect of allicin and ajoene, two compounds of garlic, on inducible nitric oxide synthase. Atherosclerosis 139:333-339.

Do GM, Kwon EY, Kim HJ, Jeon SM, Ha TY, Park T, and Choi MS (2008) Long-term effects of resveratrol supplementation on suppression of atherogenic lesion formation and cholesterol synthesis in apo E-deficient mice. Biochem Biophys Res Commun 374:55-59.

Domschke G, Linden F, Pawig L, Hafner A, Akhavanpoor M, Reymann J, Doesch AO, Erbel C, Weber C, Katus HA, et al. (2018) Systematic RNA-interference in primary human monocyte-derived macrophages: a high-throughput platform to study foam cell formation. Sci Rep 8:10516.

Dong B, Li H, Singh AB, Cao A, and Liu J (2015) Inhibition of PCSK9 transcription by berberine involves down-regulation of hepatic HNF1 $\alpha$ protein expression through the ubiquitin-proteasome degradation pathway. $J$ Biol Chem 290: 4047-4058.

Dong W, Wang X, Bi S, Pan Z, Liu S, Yu H, Lu H, Lin X, Wang X, Ma T, et al. (2014) Inhibitory effects of resveratrol on foam cell formation are mediated through monocyte chemotactic protein-1 and lipid metabolism-related proteins. Int J Mol Med 33:1161-1168

Dory L (1991) Regulation of apolipoprotein E secretion by high density lipoprotein 3 in mouse macrophages. J Lipid Res 32:783-792.

Draude G, Hrboticky N, and Lorenz RL (1999) The expression of the lectin-like oxidized low-density lipoprotein receptor (LOX-1) on human vascular smooth muscle cells and monocytes and its down-regulation by lovastatin. Biochem Pharmacol $\mathbf{5 7}$ $383-386$.

Driscoll DM and Getz GS (1984) Extrahepatic synthesis of apolipoprotein E. J Lipid Res 25:1368-1379.

Du C, Shi Y, Ren Y, Wu H, Yao F, Wei J, Wu M, Hou Y, and Duan H (2015) Anthocyanins inhibit high-glucose-induced cholesterol accumulation and inflammation by activating LXR $\alpha$ pathway in HK-2 cells. Drug Des Devel Ther 9: 5099-5113 
Du H, Schiavi S, Wan N, Levine M, Witte DP, and Grabowski GA (2004) Reduction of atherosclerotic plaques by lysosomal acid lipase supplementation. Arterioscler Thromb Vasc Biol 24:147-154.

Du J, Sun LN, Xing WW, Huang BK, Jia M, Wu JZ, Zhang H, and Qin LP (2009) Lipid-lowering effects of polydatin from Polygonum cuspidatum in hyperlipidemic hamsters. Phytomedicine 16:652-658.

Duan L, Xiong X, Hu J, Liu Y, Li J, and Wang J (2017) Panax notoginseng saponins for treating coronary artery disease: a functional and mechanistic overview. Front Pharmacol 8:702.

Duangjai A, Ingkaninan K, Praputbut S, and Limpeanchob N (2013) Black pepper and piperine reduce cholesterol uptake and enhance translocation of cholesterol transporter proteins. J Nat Med 67:303-310.

Dubland JA and Francis GA (2015) Lysosomal acid lipase: at the crossroads of normal and atherogenic cholesterol metabolism. Front Cell Dev Biol 3:3

Duchnowicz P, Broncel M, Podsędek A, and Koter-Michalak M (2012) Hypolipidemic and antioxidant effects of hydroxycinnamic acids, quercetin, and cyanidin 3-glucoside in hypercholesterolemic erythrocytes (in vitro study). Eur J Nutr 51:435-443.

Duffy D and Rader DJ (2006) Emerging therapies targeting high-density lipoprotein metabolism and reverse cholesterol transport. Circulation 113:1140-1150.

Dunn S, Vohra RS, Murphy JE, Homer-Vanniasinkam S, Walker JH, and Ponnambalam S (2008) The lectin-like oxidized low-density-lipoprotein receptor: a pro-inflammatory factor in vascular disease. Biochem J 409:349-355.

Duverger N, Kruth H, Emmanuel F, Caillaud JM, Viglietta C, Castro G, Tailleux A Fievet C, Fruchart JC, Houdebine LM, et al. (1996) Inhibition of atherosclerosis development in cholesterol-fed human apolipoprotein A-I-transgenic rabbits. Circulation 94:713-717.

Duwensee K, Schwaiger S, Tancevski I, Eller K, van Eck M, Markt P, Linder T, Stanzl U, Ritsch A, Patsch JR, et al. (2011) Leoligin, the major lignan from Edelweiss, activates cholesteryl ester transfer protein. Atherosclerosis 219:109-115.

Easton R, Gille A, D'Andrea D, Davis R, Wright SD, and Shear C (2014) A multiple ascending dose study of CSL112, an infused formulation of ApoA-I. J Clin Pharmacol 54:301-310

Eguchi A, Kaneko Y, Murakami A, and Ohigashi H (2007) Zerumbone suppresses phorbol ester-induced expression of multiple scavenger receptor genes in THP-1 human monocytic cells. Biosci Biotechnol Biochem 71:935-945.

El Bouhassani M, Gilibert S, Moreau M, Saint-Charles F, Tréguier M, Poti F, Chapman MJ, Le Goff W, Lesnik P, and Huby T (2011) Cholesteryl ester transfer protein expression partially attenuates the adverse effects of SR-BI receptor deficiency on cholesterol metabolism and atherosclerosis. $J$ Biol Chem 286: 17227-17238.

Elomaa O, Sankala M, Pikkarainen T, Bergmann U, Tuuttila A, Raatikainen-Ahokas A, Sariola H, and Tryggvason K (1998) Structure of the human macrophage MARCO receptor and characterization of its bacteria-binding region. $J$ Biol Chem 273:4530-4538.

Emini Veseli B, Perrotta P, De Meyer GRA, Roth L, Van der Donckt C, Martinet W, and De Meyer GRY (2017) Animal models of atherosclerosis. Eur J Pharmacol 816: $3-13$.

Endemann G, Stanton LW, Madden KS, Bryant CM, White RT, and Protter AA (1993) CD36 is a receptor for oxidized low density lipoprotein. J Biol Chem 268: 11811-11816.

Escary JL, Choy HA, Reue K, and Schotz MC (1998) Hormone-sensitive lipase overexpression increases cholesteryl ester hydrolysis in macrophage foam cells. Arterioscler Thromb Vasc Biol 18:991-998.

Escary JL, Choy HA, Reue K, Wang XP, Castellani LW, Glass CK, Lusis AJ, and Schotz MC (1999) Paradoxical effect on atherosclerosis of hormone-sensitive lipase overexpression in macrophages. J Lipid Res 40:397-404.

Escher G, Krozowski Z, Croft KD, and Sviridov D (2003) Expression of sterol 27hydroxylase (CYP27A1) enhances cholesterol efflux. J Biol Chem 278: 11015-11019.

Fakhrudin N, Ladurner A, Atanasov AG, Heiss EH, Baumgartner L, Markt P, Schuster D, Ellmerer EP, Wolber G, Rollinger JM, et al. (2010) Computer-aided discovery, validation, and mechanistic characterization of novel neolignan activators of peroxisome proliferator-activated receptor gamma. Mol Pharmacol 77: 559-566.

Fam TK, Klymchenko AS, and Collot M (2018) Recent advances in fluorescent probes for lipid droplets. Materials (Basel) 11:E1768.

Fang J, Little PJ, and Xu S (2018) Atheroprotective effects and molecular targets of tanshinones derived from herbal medicine danshen. Med Res Rev 38 $201-228$

Fang J and Zhang Y (2017) Icariin, an anti-atherosclerotic drug from Chinese medicinal herb horny goat weed. Front Pharmacol 8:734.

Farese RV Jr (2006) The nine lives of ACAT inhibitors. Arterioscler Thromb Vasc Biol 26:1684-1686

Fazio S, Babaev VR, Murray AB, Hasty AH, Carter KJ, Gleaves LA, Atkinson JB, and Linton MF (1997) Increased atherosclerosis in mice reconstituted with apolipoprotein E null macrophages. Proc Natl Acad Sci USA 94:4647-4652.

Fazio S and Linton M (2006) Failure of ACAT inhibition to retard atherosclerosis. $N$ Engl J Med 354:1307-1309.

Fazio S, Major AS, Swift LL, Gleaves LA, Accad M, Linton MF, and Farese RV Jr (2001) Increased atherosclerosis in LDL receptor-null mice lacking ACAT1 in macrophages. J Clin Invest 107:163-171.

Febbraio M, Hajjar DP, and Silverstein RL (2001) CD36: a class B scavenger receptor involved in angiogenesis, atherosclerosis, inflammation, and lipid metabolism. $J$ Clin Invest 108:785-791.

Febbraio M, Podrez EA, Smith JD, Hajjar DP, Hazen SL, Hoff HF, Sharma K, and Silverstein RL (2000) Targeted disruption of the class B scavenger receptor CD36 protects against atherosclerotic lesion development in mice. J Clin Invest 105:1049-1056.

Feng M, Zou Z, Zhou X, Hu Y, Ma H, Xiao Y, Li X, and Ye X (2017) Comparative effect of berberine and its derivative 8-cetylberberine on attenuating atherosclerosis in $\mathrm{ApoE}^{-/-}$mice. Int Immunopharmacol 43:195-202.
Feng X, Qin H, Shi Q, Zhang Y, Zhou F, Wu H, Ding S, Niu Z, Lu Y, and Shen P (2014) Chrysin attenuates inflammation by regulating M1/M2 status via activating PPAR $\gamma$. Biochem Pharmacol 89:503-514.

Feng X, Sureda A, Jafari S, Memariani Z, Tewari D, Annunziata G, Barrea L, Hassan STS, Smejkal K, Malaník M, et al. (2019) Berberine in cardiovascular and metabolic diseases: from mechanisms to therapeutics. Theranostics 9:1923-1951.

Feng Y, Schouteden S, Geenens R, Van Duppen V, Herijgers P, Holvoet P, Van Veldhoven PP, and Verfaillie CM (2012) Hematopoietic stem/progenitor cell proliferation and differentiation is differentially regulated by high-density and lowdensity lipoproteins in mice. PLoS One 7:e47286.

Ference BA, Ginsberg HN, Graham I, Ray KK, Packard CJ, Bruckert E, Hegele RA Krauss RM, Raal FJ, Schunkert H, et al. (2017) Low-density lipoproteins cause atherosclerotic cardiovascular disease. 1. Evidence from genetic, epidemiologic, and clinical studies. A consensus statement from the European Atherosclerosis Society Consensus Panel. Eur Heart $J$ 38:2459-2472.

Ferretti G, Bacchetti T, Nègre-Salvayre A, Salvayre R, Dousset N, and Curatola G (2006) Structural modifications of HDL and functional consequences. Atherosclerosis 184:1-7.

Fiedor J and Burda K (2014) Potential role of carotenoids as antioxidants in human health and disease. Nutrients 6:466-488.

Fitzgerald ML, Morris AL, Rhee JS, Andersson LP, Mendez AJ, and Freeman MW (2002) Naturally occurring mutations in the largest extracellular loops of ABCA1 can disrupt its direct interaction with apolipoprotein A-I. J Biol Chem 277: 33178-33187.

Franck G, Even G, Gautier A, Salinas M, Loste A, Procopio E, Gaston AT, Morvan M, Dupont S, Deschildre C, et al. (2019) Haemodynamic stress-induced breaches of the arterial intima trigger inflammation and drive atherogenesis. Eur Heart $J$ 40: 928-937.

Frolov A, Petrescu A, Atshaves BP, So PT, Gratton E, Serrero G, and Schroeder F (2000) High density lipoprotein-mediated cholesterol uptake and targeting to lipid droplets in intact L-cell fibroblasts. A single- and multiphoton fluorescence approach. J Biol Chem 275:12769-12780.

Fruchart JC (2013) Selective peroxisome proliferator-activated receptor $\alpha$ modulators $(\mathrm{SPPARM} \alpha)$ : the next generation of peroxisome proliferator-activated receptor $\alpha$-agonists. Cardiovasc Diabetol 12:82.

Fu X, Xu AG, Yao MY, Guo L, and Zhao LS (2014) Emodin enhances cholesterol efflux by activating peroxisome proliferator-activated receptor- $\gamma$ in oxidized low density lipoprotein-loaded THP1 macrophages. Clin Exp Pharmacol Physiol 41:679-684.

Fuhrman B, Elis A, and Aviram M (1997) Hypocholesterolemic effect of lycopene and beta-carotene is related to suppression of cholesterol synthesis and augmentation of LDL receptor activity in macrophages. Biochem Biophys Res Commun 233 $658-662$.

Fujiwara Y, Kiyota N, Tsurushima K, Yoshitomi M, Horlad H, Ikeda T, Nohara T, Takeya M, and Nagai R (2012) Tomatidine, a tomato sapogenol, ameliorates hyperlipidemia and atherosclerosis in apoE-deficient mice by inhibiting acyl-CoA: cholesterol acyl-transferase (ACAT). J Agric Food Chem 60:2472-2479.

Fukao H, Ijiri Y, Miura M, Hashimoto M, Yamashita T, Fukunaga C, Oiwa K, Kawai Y, Suwa M, and Yamamoto J (2004) Effect of trans-resveratrol on the thrombogenicity and atherogenicity in apolipoprotein E-deficient and low-density lipoprotein receptor-deficient mice. Blood Coagul Fibrinolysis 15:441-446.

Fukuhara-Takaki K, Sakai M, Sakamoto Y, Takeya M, and Horiuchi S (2005) Expression of class A scavenger receptor is enhanced by high glucose in vitro and under diabetic conditions in vivo: one mechanism for an increased rate of atherosclerosis in diabetes. J Biol Chem 280:3355-3364.

Fullerton MD, Ford RJ, McGregor CP, LeBlond ND, Snider SA, Stypa SA, Day EA, Lhoták S. Schertzer JD, Austin RC, et al. (2015) Salicylate improves macrophage cholesterol homeostasis via activation of Ampk. J Lipid Res 56:1025-1033.

Ganjali S, Ricciuti B, Pirro M, Butler AE, Atkin SL, Banach M, and Sahebkar A (2019) High-density lipoprotein components and functionality in cancer: state-ofthe-art. Trends Endocrinol Metab 30:12-24.

Gao H, Li L, Li L, Gong B, Dong P, Fordjour PA, Zhu Y, and Fan G (2016) Danshensu promotes cholesterol efflux in RAW264.7 macrophages. Lipids 51:1083-1092.

Gao J, Xu Y, Yang Y, Yang Y, Zheng Z, Jiang W, Hong B, Yan X, and Si S (2008) Identification of upregulators of human ATP-binding cassette transporter A1 via high-throughput screening of a synthetic and natural compound library. J Biomol Screen 13:648-656.

Gao S, Liu Z, Li H, Little PJ, Liu P, and Xu S (2012) Cardiovascular actions and therapeutic potential of tanshinone IIA. Atherosclerosis 220:3-10.

Garber DW, Venkatachalapathi YV, Gupta KB, Ibdah J, Phillips MC, Hazelrig JB Segrest JP, and Anantharamaiah GM (1992) Turnover of synthetic class A amphipathic peptide analogues of exchangeable apolipoproteins in rats. Correlation with physical properties. Arterioscler Thromb 12:886-894.

Gast M, Rauch BH, Nakagawa S, Haghikia A, Jasina A, Haas J, Nath N, Jensen L, Stroux A, Bohm A, et al. (2019) Immune system-mediated atherosclerosis caused by deficiency of long non-coding RNA MALAT1 in ApoE-/-mice. Cardiovasc Res 115:302-314.

Gelissen IC, Harris M, Rye KA, Quinn C, Brown AJ, Kockx M, Cartland S, Packianathan M, Kritharides L, and Jessup W (2006) ABCA1 and ABCG1 synergize to mediate cholesterol export to apoA-I. Arterioscler Thromb Vasc Biol 26: $534-540$

Geng J, Yang C, Wang B, Zhang X, Hu T, Gu Y, and Li J (2018) Trimethylamine $\mathrm{N}$-oxide promotes atherosclerosis via CD36-dependent MAPK/JNK pathway. Biomed Pharmacother 97:941-947.

Getz GS and Reardon CA (2012) Animal models of atherosclerosis. Arterioscler Thromb Vasc Biol 32:1104-1115.

Ghelardini C, Galeotti N, Lelli C, and Bartolini A (2001) M1 receptor activation is a requirement for arecoline analgesia. Farmaco 56:383-385.

Ghosh S (2000) Cholesteryl ester hydrolase in human monocyte/macrophage: cloning, sequencing, and expression of full-length cDNA. Physiol Genomics 2:1-8.

Giau VV, Bagyinszky E, An SSA, and Kim SY (2015) Role of apolipoprotein E in neurodegenerative diseases. Neuropsychiatr Dis Treat 11:1723-1737. 
Gobalakrishnan S, Asirvatham SS, and Janarthanam V (2016) Effect of silybin on lipid profile in hypercholesterolaemic rats. J Clin Diagn Res 10:FF01-FF05.

Goedeke L, Rotllan N, Canfrán-Duque A, Aranda JF, Ramírez CM, Araldi E, Lin CS, Anderson NN, Wagschal A, de Cabo R, et al. (2015) MicroRNA-148a regulates LDL receptor and ABCA1 expression to control circulating lipoprotein levels. Nat Med 21:1280-1289.

Goffinet M, Tardy C, Bluteau A, Boubekeur N, Baron R, Keyserling C, Barbaras R, Lalwani N, and Dasseux JL (2012) Anti-atherosclerotic effect of CER-001, an engineered HDL-mimetic, in the high-fat diet-fed LDLr knockout mice (Abstract). Circulation 126:A18667.

Goldstein JL, Ho YK, Basu SK, and Brown MS (1979) Binding site on macrophages that mediates uptake and degradation of acetylated low density lipoprotein, producing massive cholesterol deposition. Proc Natl Acad Sci USA 76:333-337.

Gonen A, Harats D, Rabinkov A, Miron T, Mirelman D, Wilchek M, Weiner L, Ulman E, Levkovitz H, Ben-Shushan D, et al. (2005) The antiatherogenic effect of allicin: possible mode of action. Pathobiology 72:325-334.

Gong G, Qin Y, and Huang W (2011) Anti-thrombosis effect of diosgenin extract from Dioscorea zingiberensis C.H. Wright in vitro and in vivo. Phytomedicine 18 458-463.

Gong G, Qin Y, Huang W, Zhou S, Wu X, Yang X, Zhao Y, and Li D (2010) Protective effects of diosgenin in the hyperlipidemic rat model and in human vascular endothelial cells against hydrogen peroxide-induced apoptosis. Chem Biol Interact 184:366-375

Gordon A (2003) Red clover extracts not effective for hot flushes. J Fam Pract 52: 846-847.

Goswami R, Merth M, Sharma S, Alharbi MO, Aranda-Espinoza H, Zhu X, and Rahaman SO (2017) TRPV4 calcium-permeable channel is a novel regulator of oxidized LDL-induced macrophage foam cell formation. Free Radic Biol Med 110 $142-150$

Goto T, Takahashi N, Hirai S, and Kawada T (2010) Various terpenoids derived from herbal and dietary plants function as PPAR modulators and regulate carbohydrate and lipid metabolism. PPAR Res 2010:483958.

Graversen JH, Laurberg JM, Andersen MH, Falk E, Nieland J, Christensen J, Etzerodt M, Thøgersen HC, and Moestrup SK (2008) Trimerization of apolipoprotein A-I retards plasma clearance and preserves antiatherosclerotic properties. $J$ Cardiovasc Pharmacol 51:170-177.

Greaves DR and Gordon S (2005) Thematic review series: the immune system and atherogenesis. Recent insights into the biology of macrophage scavenger receptors. J Lipid Res 46:11-20.

Greenspan P, Mayer EP, and Fowler SD (1985) Nile red: a selective fluorescent stain for intracellular lipid droplets. J Cell Biol 100:965-973.

Gu HF, Li HZ, Tang YL, Tang XQ, Zheng XL, and Liao DF (2016) Nicotinatecurcumin impedes foam cell formation from THP-1 cells through restoring autophagy flux. PLoS One 11:e0154820.

Guan S, Wang B, Li W, Guan J, and Fang X (2010) Effects of berberine on expression of LOX-1 and SR-BI in human macrophage-derived foam cells induced by ox-LDL. Am J Chin Med 38:1161-1169.

Gugliandolo E, Fusco R, Biundo F, D'Amico R, Benedetto F, Di Paola R, and Cuzzocrea S (2017) Palmitoylethanolamide and polydatin combination reduces inflammation and oxidative stress in vascular injury. Pharmacol Res 123:83-92.

Gui YZ, Yan H, Gao F, Xi C, Li HH, and Wang YP (2016) Betulin attenuates atherosclerosis in apoE ${ }^{-/-}$mice by up-regulating ABCA1 and ABCG1. Acta Pharmacol Sin 37:1337-1348.

Guo HX, Liu DH, Ma Y, Liu JF, Wang Y, Du ZY, Wang X, Shen JK, and Peng HL (2009) Long-term baicalin administration ameliorates metabolic disorders and hepatic steatosis in rats given a high-fat diet. Acta Pharmacol Sin 30:1505-1512.

Guo Y, Wang QZ, Tang BS, Zuo YF, Li FM, Jiang X, Wang L, and Ma KF (2006) Effects of aspirin on atherosclerosis and the cyclooxygenase-2 expression in atherosclerotic rabbits. Chin Med J (Engl) 119:1808-1814.

Haidar B, Denis M, Krimbou L, Marcil M, and Genest J Jr (2002) cAMP induces ABCA1 phosphorylation activity and promotes cholesterol efflux from fibroblasts. J Lipid Res 43:2087-2094.

Haidar B, Denis M, Marcil M, Krimbou L, and Genest J Jr (2004) Apolipoprotein A-I activates cellular cAMP signaling through the ABCA1 transporter. $J$ Biol Chem 279:9963-9969.

Han YH, Kee JY, Park J, Kim HL, Jeong MY, Kim DS, Jeon YD, Jung Y, Youn DH, Kang J, et al. (2016) Arctigenin inhibits adipogenesis by inducing AMPK activation and reduces weight gain in high-fat diet-induced obese mice. J Cell Biochem 117 2067-2077.

Handberg A, Skjelland M, Michelsen AE, Sagen EL, Krohg-Sørensen K, Russell D, Dahl A, Ueland T, Oie E, Aukrust P, et al. (2008) Soluble CD36 in plasma is increased in patients with symptomatic atherosclerotic carotid plaques and is related to plaque instability. Stroke 39:3092-3095.

Hang Y, Qin X, Ren T, and Cao J (2018) Baicalin reduces blood lipids and inflammation in patients with coronary artery disease and rheumatoid arthritis: a randomized, double-blind, placebo-controlled trial. Lipids Health Dis 17:146.

Hansson GK (2005) Inflammation, atherosclerosis, and coronary artery disease. $N$ Engl $J$ Med 352:1685-1695.

Hansson GK and Hermansson A (2011) The immune system in atherosclerosis. Nat Immunol 12:204-212.

Hansson GK, Robertson AK, and Söderberg-Nauclér C (2006) Inflammation and atherosclerosis. Annu Rev Pathol 1:297-329.

Hao S, Xiao Y, Lin Y, Mo Z, Chen Y, Peng X, Xiang C, Li Y, and Li W (2016) Chlorogenic acid-enriched extract from Eucommia ulmoides leaves inhibits hepatic lipid accumulation through regulation of cholesterol metabolism in HepG2 cells. Pharm Biol 54:251-259.

Harari A, Abecassis R, Relevi N, Levi Z, Ben-Amotz A, Kamari Y, Harats D, and Shaish A (2013) Prevention of atherosclerosis progression by 9 -cis- $\beta$-carotene rich alga Dunaliella in apoE-deficient mice. BioMed Res Int 2013:169517.

Harari A, Harats D, Marko D, Cohen H, Barshack I, Kamari Y, Gonen A, Gerber Y, Ben-Amotz A, and Shaish A (2008) A 9-cis beta-carotene-enriched diet inhibits atherogenesis and fatty liver formation in LDL receptor knockout mice. J Nutr 138 : $1923-1930$

Hardy LM, Frisdal E, and Le Goff W (2017) Critical role of the human ATP-binding cassette G1 transporter in cardiometabolic diseases. Int J Mol Sci 18:E1892.

Hasan ST, Zingg JM, Kwan P, Noble T, Smith D, and Meydani M (2014) Curcumin modulation of high fat diet-induced atherosclerosis and steatohepatosis in LDL receptor deficient mice. Atherosclerosis 232:40-51.

Hashizume M and Mihara M (2012) Atherogenic effects of TNF- $\alpha$ and IL-6 via upregulation of scavenger receptors. Cytokine 58:424-430.

Hayashi T, Juliet PA, Matsui-Hirai H, Miyazaki A, Fukatsu A, Funami J, Iguchi A, and Ignarro LJ (2005) l-Citrulline and l-arginine supplementation retards the progression of high-cholesterol-diet-induced atherosclerosis in rabbits. Proc Natl Acad Sci USA 102:13681-13686.

Hayashi T, Matsui-Hirai H, Miyazaki-Akita A, Fukatsu A, Funami J, Ding QF, Kamalanathan S, Hattori Y, Ignarro LJ, and Iguchi A (2006) Endothelial cellular senescence is inhibited by nitric oxide: implications in atherosclerosis associated with menopause and diabetes. Proc Natl Acad Sci USA 103:17018-17023.

Hayden JM, Brachova L, Higgins K, Obermiller L, Sevanian A, Khandrika S, and Reaven PD (2002) Induction of monocyte differentiation and foam cell formation in vitro by 7-ketocholesterol. J Lipid Res 43:26-35.

He D, Wang H, Xu L, Wang X, Peng K, Wang L, Liu P, and Qu P (2016a) Saikosaponin-a attenuates oxidized LDL uptake and prompts cholesterol efflux in THP-1 cells. J Cardiovasc Pharmacol 67:510-518.

He K, Li Y, Yang K, Gong JP, and Li PZ (2014) Effect of peroxisome proliferatoractivated receptor $\gamma$ on the cholesterol efflux of peritoneal macrophages in inflammation. Mol Med Rep 10:373-378.

He W, Li Y, Tang J, Luan F, Jin J, and Hu Z (2006) Comparison of the characterization on binding of alpinetin and cardamonin to lysozyme by spectroscopic methods. Int J Biol Macromol 39:165-173.

He W, Li Y, Xue C, Hu Z, Chen X, and Sheng F (2005) Effect of Chinese medicine alpinetin on the structure of human serum albumin. Bioorg Med Chem 13: $1837-1845$

He XW, Yu D, Li WL, Zheng Z, Lv CL, Li C, Liu P, Xu CQ, Hu XF, and Jin XP (2016b) Anti-atherosclerotic potential of baicalin mediated by promoting cholesterol efflux from macrophages via the PPAR $\gamma-\mathrm{LXR} \alpha-\mathrm{ABCA} 1 / \mathrm{ABCG} 1$ pathway. Biomed Pharmacother 83:257-264.

He Y, Fan Q, Cai T, Huang W, Xie X, Wen Y, and Shi Z (2018) Molecular mechanisms of the action of arctigenin in cancer. Biomed Pharmacother 108:403-407.

Hei ZQ, Huang HQ, Tan HM, Liu PQ, Zhao LZ, Chen SR, Huang WG, Chen FY, and Guo FF (2006) Emodin inhibits dietary induced atherosclerosis by antioxidation and regulation of the sphingomyelin pathway in rabbits. Chin Med $J$ (Engl) 119:868-870.

Hemn HO, Hazilawati H, Noordin MM, Heshu SR, and Zuki ABZ (2013) Influence of zerumbone supplementation a natural dietary product from zingiber zerumbet smith on early-developed atherosclerotic lesions in cholesterol. Open Conf Proc J 4 61-64.

Hemn HO, Noordin MM, Rahman HS, Hazilawati H, Zuki A, and Chartrand MS (2015) Antihypercholesterolemic and antioxidant efficacies of zerumbone on the formation, development, and establishment of atherosclerosis in cholesterol-fed rabbits. Drug Des Devel Ther 9:4173-4208.

Herijgers N, de Winther MP, Van Eck M, Havekes LM, Hofker MH, Hoogerbrugge PM, and Van Berkel TJ (2000) Effect of human scavenger receptor class A overexpression in bone marrow-derived cells on lipoprotein metabolism and atherosclerosis in low density lipoprotein receptor knockout mice. J Lipid Res 41 1402-1409.

Hermonat PL, Zhu H, Cao M, and Mehta JL (2011) LOX-1 transcription. Cardiovasc Drugs Ther 25:393-400.

Hewing B, Parathath S, Barrett T, Chung WK, Astudillo YM, Hamada T, Ramkhelawon B, Tallant TC, Yusufishaq MS, Didonato JA, et al. (2014) Effects of native and myeloperoxidase-modified apolipoprotein a-I on reverse cholesterol transport and atherosclerosis in mice. Arterioscler Thromb Vasc Biol 34:779-789.

Hidalgo M, Martin-Santamaria S, Recio I, Sanchez-Moreno C, de Pascual-Teresa B, Rimbach G, and de Pascual-Teresa S (2012) Potential anti-inflammatory, antiadhesive, anti/estrogenic, and angiotensin-converting enzyme inhibitory activities of anthocyanins and their gut metabolites. Genes Nutr 7:295-306.

Hiebl V, Ladurner A, Latkolik S, and Dirsch VM (2018) Natural products as modulators of the nuclear receptors and metabolic sensors LXR, FXR and RXR. Biotechnol Adv 36:1657-1698.

Hilgendorf I and Swirski FK (2012) Making a difference: monocyte heterogeneity in cardiovascular disease. Curr Atheroscler Rep 14:450-459.

Ho SE, Ide N, and Lau BH (2001) S-allyl cysteine reduces oxidant load in cells involved in the atherogenic process. Phytomedicine 8:39-46.

Hoang MH, Jia Y, Jun HJ, Lee JH, Lee BY, and Lee SJ (2012) Fucosterol is a selective liver $\mathrm{X}$ receptor modulator that regulates the expression of key genes in cholesterol homeostasis in macrophages, hepatocytes, and intestinal cells. J Agric Food Chem 60:11567-11575.

Hofmann A, Brunssen C, Poitz DM, Langbein H, Strasser RH, Henle T, Ravens U, and Morawietz H (2017) Lectin-like oxidized low-density lipoprotein receptor-1 promotes endothelial dysfunction in LDL receptor knockout background. Atheroscler Suppl 30:294-302.

Holy EW, Akhmedov A, Speer T, Camici GG, Zewinger S, Bonetti N, Beer JH Lüscher TF, and Tanner FC (2016) Carbamylated low-density lipoproteins induce a prothrombotic state via LOX-1: impact on arterial thrombus formation in vivo. $J$ Am Coll Cardiol 68:1664-1676.

Hong Z, Xu Y, Yin JF, Jin J, Jiang Y, and Du Q (2014) Improving the effectiveness of (-)-epigallocatechin gallate (EGCG) against rabbit atherosclerosis by EGCG-loaded nanoparticles prepared from chitosan and polyaspartic acid. J Agric Food Chem $\mathbf{6 2}$ 12603-12609.

Hopkins PN (2013) Molecular biology of atherosclerosis. Physiol Rev 93:1317-1542.

Horie T, Ono K, Horiguchi M, Nishi H, Nakamura T, Nagao K, Kinoshita M, Kuwabara Y, Marusawa H, Iwanaga Y, et al. (2010) MicroRNA-33 encoded by an 
intron of sterol regulatory element-binding protein 2 (Srebp2) regulates HDL in vivo. Proc Natl Acad Sci USA 107:17321-17326.

Horvai A, Palinski W, Wu H, Moulton KS, Kalla K, and Glass CK (1995) Scavenger receptor A gene regulatory elements target gene expression to macrophages and to foam cells of atherosclerotic lesions. Proc Natl Acad Sci USA 92:5391-5395.

Hovingh GK, Brownlie A, Bisoendial RJ, Dube MP, Levels JH, Petersen W, Dullaart RP, Stroes ES, Zwinderman AH, de Groot E, et al. (2004) A novel apoA-I mutation (L178P) leads to endothelial dysfunction, increased arterial wall thickness, and premature coronary artery disease. J Am Coll Cardiol 44:1429-1435.

HPS2-THRIVE Collaborative Group (2013) HPS2-THRIVE randomized placebocontrolled trial in 25673 high-risk patients of ER niacin/laropiprant: trial design, pre-specified muscle and liver outcomes, and reasons for stopping study treatment. Eur Heart $J$ 34:1279-1291.

Hsieh V, Kim MJ, Gelissen IC, Brown AJ, Sandoval C, Hallab JC, Kockx M, Traini M, Jessup W, and Kritharides L (2014) Cellular cholesterol regulates ubiquitination and degradation of the cholesterol export proteins ABCA1 and ABCG1. J Biol Chem 289:7524-7536.

Hu C, Dandapat A, Sun L, Chen J, Marwali MR, Romeo F, Sawamura T, and Mehta JL (2008) LOX-1 deletion decreases collagen accumulation in atherosclerotic plaque in low-density lipoprotein receptor knockout mice fed a high-cholesterol diet. Cardiovasc Res 79:287-293.

Hu K, Yang Y, Tu Q, Luo Y, and Ma R (2013a) Alpinetin inhibits LPS-induced inflammatory mediator response by activating PPAR- $\gamma$ in THP-1-derived macrophages. Eur J Pharmacol 721:96-102.

Hu WJ, Zhang Z, and Dai M (2016a) Paeonol affects proliferation activity of rat vasular endothelial cells induced by lipopolysaccharide and co-cultured with smooth muscle cells via inhibiting pathway of PI3K/AKT-NF-кB signaling. Zhongguo Zhong Yao Za Zhi 41:2298-2302.

Hu Y, Sun B, Liu K, Yan M, Zhang Y, Miao C, and Ren L (2016b) Icariin attenuates high-cholesterol diet induced atherosclerosis in rats by inhibition of inflammatory response and p38 MAPK signaling pathway. Inflammation 39:228-236.

Hu YW, Yang JY, Ma X, Chen ZP, Hu YR, Zhao JY, Li SF, Qiu YR, Lu JB, Wang YC, et al. (2014) A lincRNA-DYNLRB2-2/GPR119/GLP-1R/ABCA1-dependent signal transduction pathway is essential for the regulation of cholesterol homeostasis. J Lipid Res 55:681-697.

Hu YW, Zhao JY, Li SF, Huang JL, Qiu YR, Ma X, Wu SG, Chen ZP, Hu YR, Yang JY, et al. (2015) RP5-833A20.1/miR-382-5p/NFIA-dependent signal transduction pathway contributes to the regulation of cholesterol homeostasis and inflammatory reaction. Arterioscler Thromb Vasc Biol 35:87-101.

$\mathrm{Hu}$ Z, Hu J, Zhang Z, Shen WJ, Yun CC, Berlot CH, Kraemer FB, and Azhar S (2013b) Regulation of expression and function of scavenger receptor class B, type I (SR-BI) by $\mathrm{Na}+/ \mathrm{H}+$ exchanger regulatory factors (NHERFs). $J$ Biol Chem 288 11416-11435.

Hu Z, Shen WJ, Kraemer FB, and Azhar S (2012) MicroRNAs 125a and 455 repress lipoprotein-supported steroidogenesis by targeting scavenger receptor class B type I in steroidogenic cells. Mol Cell Biol 32:5035-5045.

Huang EW, Liu CZ, Liang SJ, Zhang Z, Lv XF, Liu J, Zhou JG, Tang YB, and Guan YY (2016a) Endophilin-A2-mediated increase in scavenger receptor expression contributes to macrophage-derived foam cell formation. Atherosclerosis $\mathbf{2 5 4}$ 133-141.

Huang L, Chambliss KL, Gao X, Yuhanna IS, Behling-Kelly E, Bergaya S, Ahmed M, Michaely P, Luby-Phelps K, Darehshouri A, et al. (2019) SR-B1 drives endothelial cell LDL transcytosis via DOCK4 to promote atherosclerosis. Nature 569:565-569.

Huang LH, Melton EM, Li H, Sohn P, Jung D, Tsai CY, Ma T, Sano H, Ha H, Friedline RH, et al. (2018) Myeloid-specific Acat1 ablation attenuates inflammatory responses in macrophages, improves insulin sensitivity, and suppresses diet-induced obesity. Am J Physiol Endocrinol Metab 315:E340-E356.

Huang LH, Melton EM, Li H, Sohn P, Rogers MA, Mulligan-Kehoe MJ, Fiering SN, Hickey WF, Chang CC, and Chang TY (2016b) Myeloid Acyl-CoA:cholesterol acyltransferase 1 deficiency reduces lesion macrophage content and suppresses atherosclerosis progression. $J$ Biol Chem 291:6232-6244.

Huang RS, Hu GQ, Lin B, Lin ZY, and Sun CC (2010) MicroRNA-155 silencing enhances inflammatory response and lipid uptake in oxidized low-density lipoprotein-stimulated human THP-1 macrophages. J Investig Med 58:961-967.

Huang SL, Yu RT, Gong J, Feng Y, Dai YL, Hu F, Hu YH, Tao YD, and Leng Y (2012a) Arctigenin, a natural compound, activates AMP-activated protein kinase via inhibition of mitochondria complex I and ameliorates metabolic disorders in ob/ ob mice. Diabetologia 55:1469-1481.

Huang Z, Dong F, Li S, Chu M, Zhou H, Lu Z, and Huang W (2012b) Berberineinduced inhibition of adipocyte enhancer-binding protein 1 attenuates oxidized low-density lipoprotein accumulation and foam cell formation in phorbol 12 myristate 13-acetate-induced macrophages. Eur J Pharmacol 690:164-169.

Huang-Fu N, Cheng JS, Wang Y, Li ZW, and Wang SH (2018) Neat1 regulates oxidized low-density lipoprotein-induced inflammation and lipid uptake in macrophages via paraspeckle formation. Mol Med Rep 17:3092-3098.

Huangfu N, Xu Z, Zheng W, Wang Y, Cheng J, and Chen X (2018) LncRNA MALAT1 regulates oxLDL-induced CD36 expression via activating $\beta$-catenin. Biochem Biophys Res Commun 495:2111-2117.

Huwait EA, Greenow KR, Singh NN, and Ramji DP (2011) A novel role for c-Jun $\mathrm{N}$-terminal kinase and phosphoinositide 3-kinase in the liver $\mathrm{X}$ receptor-mediated induction of macrophage gene expression. Cell Signal 23:542-549.

Ibitoye OB and Ajiboye TO (2018) Dietary phenolic acids reverse insulin resistance, hyperglycaemia, dyslipidaemia, inflammation and oxidative stress in high-fructose diet-induced metabolic syndrome rats. Arch Physiol Biochem 124:410-417.

Igarashi M, Osuga J, Uozaki H, Sekiya M, Nagashima S, Takahashi M, Takase S, Takanashi M, Li Y, Ohta K, et al. (2010) The critical role of neutral cholesterol ester hydrolase 1 in cholesterol removal from human macrophages. Circ Res 107: 1387-1395.

Iio A, Ohguchi K, Iinuma M, Nozawa Y, and Ito M (2012) Hesperetin upregulates ABCA1 expression and promotes cholesterol efflux from THP-1 macrophages. $J$ Nat Prod 75:563-566.
Iizuka M, Ayaori M, Uto-Kondo H, Yakushiji E, Takiguchi S, Nakaya K, Hisada T, Sasaki M, Komatsu T, Yogo M, et al (2012) Astaxanthin enhances ATP-binding cassette transporter A1/G1 expressions and cholesterol efflux from macrophages. $J$ Nutr Sci Vitaminol (Tokyo) 58:96-104.

Ikhlef S, Berrougui H, Kamtchueng Simo O, and Khalil A (2016) Paraoxonase 1treated oxLDL promotes cholesterol efflux from macrophages by stimulating the PPAR $\gamma$-LXR $\alpha$-ABCA1 pathway. FEBS Lett 590:1614-1629.

Inoue K, Arai Y, Kurihara H, Kita T, and Sawamura T (2005) Overexpression of lectin-like oxidized low-density lipoprotein receptor-1 induces intramyocardial vasculopathy in apolipoprotein E-null mice. Circ Res 97:176-184.

Ishibashi M, Filomenko R, Rébé $\mathrm{C}$, Chevriaux A, Varin A, Derangère $\mathrm{V}$, Bessède $\mathrm{G}$, Gambert P, Lagrost L, and Masson D (2013) Knock-down of the oxysterol receptor LXR $\alpha$ impairs cholesterol efflux in human primary macrophages: lack of compensation by LXR $\beta$ activation. Biochem Pharmacol 86:122-129.

Ishii I, Yokoyama N, Yanagimachi M, Ashikawa N, Hata M, Murakami S, Asami Y, Morisaki N, Saito Y, Ohmori S, et al. (1998) Stimulation of cholesterol release from rabbit foam cells by the action of a new inhibitor for acyl CoA:cholesterol acyltransferase (ACAT), HL-004. J Pharmacol Exp Ther 287:115-121.

Ishii T, Itoh K, Ruiz E, Leake DS, Unoki H, Yamamoto M, and Mann GE (2004) Role of Nrf2 in the regulation of CD36 and stress protein expression in murine macrophages: activation by oxidatively modified LDL and 4-hydroxynonenal. Circ Res 94:609-616.

Jahagirdar R, Zhang H, Azhar S, Tobin J, Attwell S, Yu R, Wu J, McLure KG, Hansen HC, Wagner GS, et al. (2014) A novel BET bromodomain inhibitor, RVX208, shows reduction of atherosclerosis in hyperlipidemic ApoE deficient mice. Atherosclerosis 236:91-100.

Jakobsson T, Venteclef N, Toresson G, Damdimopoulos AE, Ehrlund A, Lou X, Sanyal S, Steffensen KR, Gustafsson J-Å, and Treuter E (2009) GPS2 is required for cholesterol efflux by triggering histone demethylation, LXR recruitment, and coregulator assembly at the ABCG1 locus. Mol Cell 34:510-518.

Jamal J, Mustafa MR, and Wong PF (2014) Paeonol protects against premature senescence in endothelial cells by modulating Sirtuin 1 pathway. J Ethnopharmacol 154:428-436.

Ji Y, Jian B, Wang N, Sun Y, Moya ML, Phillips MC, Rothblat GH, Swaney JB, and Tall AR (1997) Scavenger receptor BI promotes high density lipoproteinmediated cellular cholesterol efflux. J Biol Chem 272:20982-20985

Jia LQ, Zhang N, Xu Y, Chen WN, Zhu ML, Song N, Ren L, Cao HM, Wang JY, and Yang GL (2016) Tanshinone IIA affects the HDL subfractions distribution not serum lipid levels: involving in intake and efflux of cholesterol. Arch Biochem Biophys 592:50-59.

Jia Y, Li ZY, Zhang HG, Li HB, Liu Y, and Li XH (2010) Panax notoginseng saponins decrease cholesterol ester via up-regulating ATP-binding cassette transporter A1 in foam cells. $J$ Ethnopharmacol 132:297-302.

Jian B, de la Llera-Moya M, Ji Y, Wang N, Phillips MC, Swaney JB, Tall AR and Rothblat GH (1998) Scavenger receptor class B type I as a mediator of cellular cholesterol efflux to lipoproteins and phospholipid acceptors. J Biol Chem 273: $5599-5606$.

Jiang J, Mo ZC, Yin K, Zhao GJ, Lv YC, Ouyang XP, Jiang ZS, Fu Y, and Tang CK (2012) Epigallocatechin-3-gallate prevents TNF- $\alpha$-induced NF-kB activation thereby upregulating ABCA1 via the Nrf2/Keap1 pathway in macrophage foam cells. Int J Mol Med 29:946-956.

Jiang T, Ren K, Chen Q, Li H, Yao R, Hu H, Lv YC, and Zhao GJ (2017a) Leonurine prevents atherosclerosis via promoting the expression of ABCA1 and ABCG1 in a Ppar $/$ Lxr $\alpha$ signaling pathway-dependent manner. Cell Physiol Biochem 43: 1703-1717.

Jiang X, Zhao X, Chen R, Jiang Q, and Zhou B (2017b) Plasma soluble CD36, carotid intima-media thickness and cognitive function in patients with type 2 diabetes. Arch Med Sci 13:1031-1039.

Jiang Z, Sang H, Fu X, Liang Y, and Li L (2015) Alpinetin enhances cholesterol efflux and inhibits lipid accumulation in oxidized low-density lipoprotein-loaded human macrophages. Biotechnol Appl Biochem 62:840-847.

Jin FY, Kamanna VS, and Kashyap ML (1997) Niacin decreases removal of highdensity lipoprotein apolipoprotein A-I but not cholesterol ester by Hep G2 cells. Implication for reverse cholesterol transport. Arterioscler Thromb Vasc Biol 17 2020-2028.

Joffre J, Potteaux S, Zeboudj L, Loyer X, Boufenzer A, Laurans L, Esposito B, Vandestienne M, de Jager SC, Hénique C, et al. (2016) Genetic and pharmacological inhibition of TREM-1 limits the development of experimental atherosclerosis. J Am Coll Cardiol 68:2776-2793.

Johansson JO, Gordon AF, Halliday C, and Wong NC (2014) Effects of RVX-208 on major adverse cardiac events (MACE), apolipoprotein A-I and high-density-lipoproteins; a post-hoc analysis from the pooled SUSTAIN and ASSURE clinical trials. Eur Heart $J$ 35:723-724.

Johansson L, Rudling M, Scanlan TS, Lundåsen T, Webb P, Baxter J, Angelin B, and Parini P (2005) Selective thyroid receptor modulation by GC-1 reduces serum lipids and stimulates steps of reverse cholesterol transport in euthyroid mice. Proc Natl Acad Sci USA 102:10297-10302.

Jono T, Miyazaki A, Nagai R, Sawamura T, Kitamura T, and Horiuchi S (2002) Lectin-like oxidized low density lipoprotein receptor-1 (LOX-1) serves as an endothelial receptor for advanced glycation end products (AGE). FEBS Lett 511: 170-174.

Joseph L and Robinson JG (2015) Proprotein convertase subtilisin/kexin type 9 (PCSK9) inhibition and the future of lipid lowering therapy. Prog Cardiovasc Dis 58:19-31.

Ju J, Li J, Lin Q, and Xu H (2018) Efficacy and safety of berberine for dyslipidaemias: a systematic review and meta-analysis of randomized clinical trials. Phytomedicine 50:25-34.

Jun HJ, Hoang MH, Lee JW, Yaoyao J, Lee JH, Lee DH, Lee HJ, Seo WD, Hwang BY, and Lee SJ (2012) Iristectorigenin B isolated from Belamcanda chinensis is a liver $\mathrm{X}$ receptor modulator that increases ABCA1 and ABCG1 expression in macrophage RAW 264.7 cells. Biotechnol Lett 34:2213-2221. 
Jung CG, Horike H, Cha BY, Uhm KO, Yamauchi R, Yamaguchi T, Hosono T, Iida K, Woo JT, and Michikawa M (2010) Honokiol increases ABCA1 expression level by activating retinoid X receptor beta. Biol Pharm Bull 33:1105-1111.

Jung SH, Lee YS, Lee S, Lim SS, Kim YS, Ohuchi K, and Shin KH (2003) Antiangiogenic and anti-tumor activities of isoflavonoids from the rhizomes of Belamcanda chinensis. Planta Med 69:617-622.

Jung SH, Lee YS, Lee S, Lim SS, Kim YS, and Shin KH (2002) Isoflavonoids from the rhizomes of Belamcanda chinensis and their effects on aldose reductase and sorbitol accumulation in streptozotocin induced diabetic rat tissues. Arch Pharm Res 25:306-312.

Kämmerer I, Ringseis R, Biemann R, Wen G, and Eder K (2011) 13-hydroxy linoleic acid increases expression of the cholesterol transporters ABCA1, ABCG1 and SRBI and stimulates apoA-I-dependent cholesterol efflux in RAW264.7 macrophages. Lipids Health Dis 10:222.

Kasikara C, Doran AC, Cai B, and Tabas I (2018) The role of non-resolving inflammation in atherosclerosis. J Clin Invest 128:2713-2723.

Kataoka H, Kume N, Miyamoto S, Minami M, Moriwaki H, Murase T, Sawamura T, Masaki T, Hashimoto N, and Kita T (1999) Expression of lectinlike oxidized lowdensity lipoprotein receptor-1 in human atherosclerotic lesions. Circulation 99: 3110-3117.

Kawai Y, Nishikawa T, Shiba Y, Saito S, Murota K, Shibata N, Kobayashi M, Kanayama M, Uchida K, and Terao J (2008) Macrophage as a target of quercetin glucuronides in human atherosclerotic arteries: implication in the antiatherosclerotic mechanism of dietary flavonoids. J Biol Chem 283:9424-9434.

Kelley JL, Ozment TR, Li C, Schweitzer JB, and Williams DL (2014) Scavenger receptor-A (CD204): a two-edged sword in health and disease. Crit Rev Immunol 34:241-261.

Kennedy MA, Barrera GC, Nakamura K, Baldán A, Tarr P, Fishbein MC, Frank J, Francone OL, and Edwards PA (2005) ABCG1 has a critical role in mediating cholesterol efflux to HDL and preventing cellular lipid accumulation. Cell Metab $\mathbf{1}$ 121-131.

Keyserling CH, Hunt TL, Klepp HM, Scott RA, Barbaras R, Schwendeman A, Lalwani N, and Dasseux JL (2011) CER-001, a synthetic HDL-mimetic, safely mobilizes cholesterol in healthy dyslipidemic volunteers (Abstract). Circulation 124: A15525.

Kharbanda RK, Wallace S, Walton B, Donald A, Cross JM, and Deanfield J (2005) Systemic Acyl-CoA:cholesterol acyltransferase inhibition reduces inflammation and improves vascular function in hypercholesterolemia. Circulation 111:804-807.

Khera AV, Cuchel M, de la Llera-Moya M, Rodrigues A, Burke MF, Jafri K, French BC, Phillips JA, Mucksavage ML, Wilensky RL, et al. (2011) Cholesterol efflux capacity, high-density lipoprotein function, and atherosclerosis. N Engl J Med 364:127-135.

Khera AV and Rader DJ (2013) Cholesterol efflux capacity: full steam ahead or a bump in the road? Arterioscler Thromb Vasc Biol 33:1449-1451.

Kim B, Kim JE, and Kim HS (2014) Caffeic acid induces keratinocyte differentiation by activation of PPAR- $\alpha$. J Pharm Pharmacol 66:84-92.

Kim DH, Cho YM, Lee KH, Jeong SW, and Kwon OJ (2017) Oleate protects macrophages from palmitate-induced apoptosis through the downregulation of CD36 expression. Biochem Biophys Res Commun 488:477-482.

Kim J, Yoon H, Ramírez CM, Lee SM, Hoe HS, Fernández-Hernando C, and Kim J (2012) MiR-106b impairs cholesterol efflux and increases A $\beta$ levels by repressing ABCA1 expression. Exp Neurol 235:476-483.

Kim MC, Ahn Y, Jang SY, Cho KH, Hwang SH, Lee MG, Ko JS, Park KH, Sim DS, Yoon NS, et al. (2011) Comparison of clinical outcomes of hydrophilic and lipophilic statins in patients with acute myocardial infarction. Korean J Intern Med (Korean Assoc Intern Med) 26:294-303.

Kingwell BA, Chapman MJ, Kontush A, and Miller NE (2014) HDL-targeted therapies: progress, failures and future. Nat Rev Drug Discov 13:445-464.

Kishimoto Y, Tani M, Uto-Kondo H, Iizuka M, Saita E, Sone H, Kurata H, and Kondo K (2010) Astaxanthin suppresses scavenger receptor expression and matrix metalloproteinase activity in macrophages. Eur J Nutr 49:119-126.

Kiss RS, Maric J, and Marcel YL (2005) Lipid efflux in human and mouse macrophagic cells: evidence for differential regulation of phospholipid and cholesterol efflux. J Lipid Res 46:1877-1887.

Kobayashi A, Takanezawa Y, Hirata T, Shimizu Y, Misasa K, Kioka N, Arai H, Ueda $\mathrm{K}$, and Matsuo M (2006) Efflux of sphingomyelin, cholesterol, and phosphatidylcholine by ABCG1. J Lipid Res 47:1791-1802.

Kobayashi T, Tahara Y, Matsumoto M, Iguchi M, Sano H, Murayama T, Arai H, Oida H, Yurugi-Kobayashi T, Yamashita JK, et al. (2004) Roles of thromboxane A(2) and prostacyclin in the development of atherosclerosis in apoE-deficient mice. J Clin Invest 114:784-794.

Kocher O, Yesilaltay A, Cirovic C, Pal R, Rigotti A, and Krieger M (2003) Targeted disruption of the PDZK1 gene in mice causes tissue-specific depletion of the high density lipoprotein receptor scavenger receptor class B type I and altered lipoprotein metabolism. J Biol Chem 278:52820-52825.

Kockx M, Dinnes DL, Huang KY, Sharpe LJ, Jessup W, Brown AJ, and Kritharides L (2012) Cholesterol accumulation inhibits ER to Golgi transport and protein secretion: studies of apolipoprotein E and VSVGt. Biochem $J$ 447:51-60.

Kockx M, Guo DL, Traini M, Gaus K, Kay J, Wimmer-Kleikamp S, Rentero C Burnett JR, Le Goff W, Van Eck M, et al. (2009) Cyclosporin A decreases apolipoprotein $\mathrm{E}$ secretion from human macrophages via a protein phosphatase $2 \mathrm{~B}$ dependent and ATP-binding cassette transporter A1 (ABCA1)-independent pathway. J Biol Chem 284:24144-24154.

Kockx M, Rye KA, Gaus K, Quinn CM, Wright J, Sloane T, Sviridov D, Fu Y, Sullivan D, Burnett JR, et al. (2004) Apolipoprotein A-I-stimulated apolipoprotein E secretion from human macrophages is independent of cholesterol efflux. J Biol Chem 279:25966-25977.

Kockx M, Traini M, and Kritharides L (2018) Cell-specific production, secretion, and function of apolipoprotein E. J Mol Med (Berl) 96:361-371.

Kodama T, Freeman M, Rohrer L, Zabrecky J, Matsudaira P, and Krieger M (1990) Type I macrophage scavenger receptor contains alpha-helical and collagen-like coiled coils. Nature 343:531-535.
Koelwyn GJ, Corr EM, Erbay E, and Moore KJ (2018) Regulation of macrophage immunometabolism in atherosclerosis. Nat Immunol 19:526-537.

Kohlmeier L and Hastings SB (1995) Epidemiologic evidence of a role of carotenoids in cardiovascular disease prevention. Am J Clin Nutr 62 (6 Suppl):1370S-1376S. Kong W, Wei J, Abidi P, Lin M, Inaba S, Li C, Wang Y, Wang Z, Si S, Pan H, et al. (2004) Berberine is a novel cholesterol-lowering drug working through a unique mechanism distinct from statins. Nat Med 10:1344-1351.

Koppaka V, Silvestro L, Engler JA, Brouillette CG, and Axelsen PH (1999) The structure of human lipoprotein A-I. Evidence for the "belt" model. J Biol Chem 274: 14541-14544.

Koren E, Franzen J, Fugate RD, and Alaupovic P (1990) Analysis of cholesterol ester accumulation in macrophages by the use of digital imaging fluorescence microscopy. Atherosclerosis 85:175-184.

Kosaka S, Takahashi S, Masamura K, Kanehara H, Sakai J, Tohda G, Okada E, Oida K, Iwasaki T, Hattori H, et al. (2001) Evidence of macrophage foam cell formation by very low-density lipoprotein receptor: interferon-gamma inhibition of very lowdensity lipoprotein receptor expression and foam cell formation in macrophages. Circulation 103:1142-1147.

Kotani H, Tanabe H, Mizukami H, Makishima M, and Inoue M (2010) Identification of a naturally occurring rexinoid, honokiol, that activates the retinoid $\mathrm{X}$ receptor. J Nat Prod 73:1332-1336.

Kothapalli D, Castagnino P, Rader DJ, Phillips MC, Lund-Katz S, and Assoian RK (2013) Apolipoprotein E-mediated cell cycle arrest linked to p27 and the Cox2dependent repression of miR221/222. Atherosclerosis 227:65-71.

Kotla S, Singh NK, and Rao GN (2017) ROS via BTK-p300-STAT1-PPARy signaling activation mediates cholesterol crystals-induced CD36 expression and foam cell formation. Redox Biol 11:350-364.

Kou JY, Li Y, Zhong ZY, Jiang YQ, Li XS, Han XB, Liu ZN, Tian Y, and Yang LM (2017) Berberine-sonodynamic therapy induces autophagy and lipid unloading in macrophage. Cell Death Dis 8:e2558.

Kou MC, Chiou SY, Weng CY, Wang L, Ho CT, and Wu MJ (2013) Curcuminoids distinctly exhibit antioxidant activities and regulate expression of scavenger receptors and heme oxygenase-1. Mol Nutr Food Res 57:1598-1610.

Kraft HG, Menzel HJ, Hoppichler F, Vogel W, and Utermann G (1989) Changes of genetic apolipoprotein phenotypes caused by liver transplantation. Implications for apolipoprotein synthesis. J Clin Invest 83:137-142.

Krause BR and Remaley AT (2013) Reconstituted HDL for the acute treatment of acute coronary syndrome. Curr Opin Lipidol 24:480-486.

Kruth HS (2011) Receptor-independent fluid-phase pinocytosis mechanisms for in duction of foam cell formation with native low-density lipoprotein particles. Curr Opin Lipidol 22:386-393.

Kuai R, Li D, Chen YE, Moon JJ, and Schwendeman A (2016) High-density lipoproteins: nature's multifunctional nanoparticles. ACS Nano 10:3015-3041.

Kuchibhotla S, Vanegas D, Kennedy DJ, Guy E, Nimako G, Morton RE, and Febbraio M (2008) Absence of CD36 protects against atherosclerosis in ApoE knock-out mice with no additional protection provided by absence of scavenger receptor A I/II. Cardiovasc Res 78:185-196.

Kume N, Moriwaki H, Kataoka H, Minami M, Murase T, Sawamura T, Masaki T, and Kita T (2000) Inducible expression of LOX -1, a novel receptor for oxidized LDL, in macrophages and vascular smooth muscle cells. Ann N Y Acad Sci 902: 323-327.

Kunjathoor VV, Febbraio M, Podrez EA, Moore KJ, Andersson L, Koehn S, Rhee JS, Silverstein R, Hoff HF, and Freeman MW (2002) Scavenger receptors class A-I/II and CD36 are the principal receptors responsible for the uptake of modified low density lipoprotein leading to lipid loading in macrophages. J Biol Chem 277: $49982-49988$.

Kwon EY, Do GM, Cho YY, Park YB, Jeon SM, and Choi MS (2010) Anti-atherogenic property of ferulic acid in apolipoprotein E-deficient mice fed Western diet: comparison with clofibrate. Food Chem Toxicol 48:2298-2303.

Laguna-Fernandez A, Checa A, Carracedo M, Artiach G, Petri MH, Baumgartner R, Forteza MJ, Jiang X, Andonova T, Walker ME, et al. (2018) ERV1/ChemR23 signaling protects against atherosclerosis by modifying oxidized low-density lipoprotein uptake and phagocytosis in macrophages. Circulation 138:1693-1705.

Lamon-Fava S, Diffenderfer MR, Barrett PH, Buchsbaum A, Nyaku M, Horvath KV, Asztalos BF, Otokozawa S, Ai M, Matthan NR, et al. (2008) Extended-release niacin alters the metabolism of plasma apolipoprotein (Apo) A-I and ApoB containing lipoproteins. Arterioscler Thromb Vasc Biol 28:1672-1678.

Lamon-Fava S and Micherone D (2004) Regulation of apoA-I gene expression: mechanism of action of estrogen and genistein. $J$ Lipid Res 45:106-112.

Lan X, Yan J, Ren J, Zhong B, Li J, Li Y, Liu L, Yi J, Sun Q, Yang X, et al. (2016) A novel long noncoding RNA Lnc-HC binds hnRNPA2B1 to regulate expressions of Cyp7a1 and Abca1 in hepatocytic cholesterol metabolism. Hepatology 64:58-72.

Langmann T, Liebisch G, Moehle C, Schifferer R, Dayoub R, Heiduczek S, Grandl M, Dada A, and Schmitz G (2005) Gene expression profiling identifies retinoids as potent inducers of macrophage lipid efflux. Biochim Biophys Acta 1740:155-161.

Lara-Guzman OJ, Tabares-Guevara JH, Leon-Varela YM, Älvarez RM, Roldan M, Sierra JA, Londoño-Londoño JA, and Ramirez-Pineda JR (2012) Proatherogenic macrophage activities are targeted by the flavonoid quercetin. $J$ Pharmacol Exp Ther 343:296-306.

Larrosa M, García-Conesa MT, Espín JC, and Tomás-Barberán FA (2010) Ellagitannins, ellagic acid and vascular health. Mol Aspects Med 31:513-539.

Laufs U, Banach M, Mancini GBJ, Gaudet D, Bloedon LT, Sterling LR, Kelly S, and Stroes ESG (2019) Efficacy and safety of bempedoic acid in patients with hypercholesterolemia and statin intolerance. $J$ Am Heart Assoc 8:011662.

Lee $\mathrm{CH}$ and Kim JH (2014) A review on the medicinal potentials of ginseng and ginsenosides on cardiovascular diseases. J Ginseng Res 38:161-166.

Lee JM, Robson MD, Yu LM, Shirodaria CC, Cunnington C, Kylintireas I, Digby JE Bannister T, Handa A, Wiesmann F, et al. (2009a) Effects of high-dose modifiedrelease nicotinic acid on atherosclerosis and vascular function: a randomized, placebo-controlled, magnetic resonance imaging study. J Am Coll Cardiol 54: 1787-1794. 
Lee JY, Chung J, Kim KH, An SH, Kim M, Park J, and Kwon K (2018) Fluid shear stress regulates the expression of lectin-like oxidized low density lipoprotein receptor-1 via KLF2-AP-1 pathway depending on its intensity and pattern in endothelial cells. Atherosclerosis 270:76-88.

Lee KT, Sohn IC, Kim DH, Choi JW, Kwon SH, and Park HJ (2000a) Hypoglycemic and hypolipidemic effects of tectorigenin and kaikasaponin III in the streptozotocin-lnduced diabetic rat and their antioxidant activity in vitro. Arch Pharm Res 23:461-466.

Lee RG, Willingham MC, Davis MA, Skinner KA, and Rudel LL (2000b) Differential expression of ACAT1 and ACAT2 among cells within liver, intestine, kidney, and adrenal of nonhuman primates. J Lipid Res 41:1991-2001.

Lee SM, Kim CW, Kim JK, Shin HJ, and Baik JH (2008) GCG-rich tea catechins are effective in lowering cholesterol and triglyceride concentrations in hyperlipidemic rats. Lipids 43:419-429.

Lee SM, Moon J, Cho Y, Chung JH, and Shin MJ (2013) Quercetin up-regulates expressions of peroxisome proliferator-activated receptor $\gamma$, liver X receptor $\alpha$, and ATP binding cassette transporter A1 genes and increases cholesterol efflux in human macrophage cell line. Nutr Res 33:136-143.

Lee TS, Lin CY, Tsai JY, Wu YL, Su KH, Lu KY, Hsiao SH, Pan CC, Kou YR, Hsu YP, et al. (2009b) Resistin increases lipid accumulation by affecting class A scavenger receptor, CD36 and ATP-binding cassette transporter-A1 in macrophages. Life Sci 84:97-104.

Lee TS, Pan CC, Peng CC, Kou YR, Chen CY, Ching LC, Tsai TH, Chen SF, Lyu PC, and Shyue SK (2010) Anti-atherogenic effect of berberine on LXRalpha-ABCA1dependent cholesterol efflux in macrophages. J Cell Biochem 111:104-110.

Lee WJ, Ou HC, Wu CM, Lee IT, Lin SY, Lin LY, Tsai KL, Lee SD, and Sheu WH (2009c) Sesamin mitigates inflammation and oxidative stress in endothelial cells exposed to oxidized low-density lipoprotein. J Agric Food Chem 57:11406-11417.

Lee WS, Im KR, Park YD, Sung ND, and Jeong TS (2006) Human ACAT-1 and ACAT-2 inhibitory activities of pentacyclic triterpenes from the leaves of Lycopus lucidus TURCZ. Biol Pharm Bull 29:382-384.

Lee YJ, Lee YM, Lee CK, Jung JK, Han SB, and Hong JT (2011) Therapeutic applications of compounds in the Magnolia family. Pharmacol Ther 130:157-176.

Lee YT, Lin HY, Chan YW, Li KH, To OT, Yan BP, Liu T, Li G, Wong WT, Keung W, et al. (2017) Mouse models of atherosclerosis: a historical perspective and recent advances. Lipids Health Dis 16:12.

Lei L, Xiong Y, Chen J, Yang JB, Wang Y, Yang XY, Chang CC, Song BL, Chang TY, and Li BL (2009) TNF-alpha stimulates the ACAT1 expression in differentiating monocytes to promote the CE-laden cell formation. J Lipid Res 50:1057-1067.

Leiva A, Verdejo H, Benítez ML, Martínez A, Busso D, and Rigotti A (2011) Mechanisms regulating hepatic SR-BI expression and their impact on HDL metabolism. Atherosclerosis 217:299-307.

Leng S, Iwanowycz S, Saaoud F, Wang J, Wang Y, Sergin I, Razani B, and Fan D (2016) Ursolic acid enhances macrophage autophagy and attenuates atherogenesis. J Lipid Res 57:1006-1016.

Li AC, Binder CJ, Gutierrez A, Brown KK, Plotkin CR, Pattison JW, Valledor AF, Davis RA, Willson TM, Witztum JL, et al. (2004a) Differential inhibition of macrophage foam-cell formation and atherosclerosis in mice by PPARalpha, beta/delta, and gamma. J Clin Invest 114:1564-1576.

Li CH, Gong D, Chen LY, Zhang M, Xia XD, Cheng HP, Huang C, Zhao ZW, Zheng XL, Tang XE, et al. (2017a) Puerarin promotes ABCA1-mediated cholesterol efflux and decreases cellular lipid accumulation in THP-1 macrophages. Eur J Pharmacol 811:74-86.

Li H, Dai M, and Jia W (2009a) Paeonol attenuates high-fat-diet-induced atherosclerosis in rabbits by anti-inflammatory activity. Planta Med 75:7-11.

Li H, Sureda A, Devkota HP, Pittalà V, Barreca D, Silva AS, Tewari D, Xu S, and Nabavi SM (2019) Curcumin, the golden spice in treating cardiovascular diseases. Biotechnol Adv DOI: 10.1016/j.biotechadv.2019.01.010 [published ahead of print].

Li J, Ding L, Song B, Xiao X, Qi M, Yang Q, Yang Q, Tang X, Wang Z, and Yang L (2016) Emodin improves lipid and glucose metabolism in high fat diet-induced obese mice through regulating SREBP pathway. Eur J Pharmacol 770:99-109.

Li J, Xie ZZ, Tang YB, Zhou JG, and Guan YY (2011) Ginsenoside-Rd, a purified component from panax notoginseng saponins, prevents atherosclerosis in apoE knockout mice. Eur J Pharmacol 652:104-110.

Li K, Ching D, Luk FS, and Raffai RL (2015a) Apolipoprotein E enhances microRNA$146 \mathrm{a}$ in monocytes and macrophages to suppress nuclear factor-kB-driven inflammation and atherosclerosis. Circ Res 117:e1-e11.

Li K, Yao W, Zheng X, and Liao K (2009b) Berberine promotes the development of atherosclerosis and foam cell formation by inducing scavenger receptor A expression in macrophage. Cell Res 19:1006-1017.

Li L, Sawamura T, and Renier G (2003) Glucose enhances endothelial LOX-1 expression: role for LOX-1 in glucose-induced human monocyte adhesion to endothelium. Diabetes 52:1843-1850.

Li L, Sawamura T, and Renier G (2004b) Glucose enhances human macrophage LOX1 expression: role for LOX-1 in glucose-induced macrophage foam cell formation. Circ Res 94:892-901.

Li L, Shen C, Huang YX, Li YN, Liu XF, Liu XM, and Liu JH (2018a) A new strategy for rapidly screening natural inhibitors targeting the PCSK9/LDLR interaction in vitro. Molecules 23:E2397.

Li S, Jiao Y, Wang H, Shang Q, Lu F, Huang L, Liu J, Xu H, and Chen K (2017b) Sodium tanshinone IIA sulfate adjunct therapy reduces high-sensitivity C-reactive protein level in coronary artery disease patients: a randomized controlled trial. $\mathrm{Sci}$ Rep 7:17451.

Li W, Hellsten A, Jacobsson LS, Blomqvist HM, Olsson AG, and Yuan XM (2004c) Alpha-tocopherol and astaxanthin decrease macrophage infiltration, apoptosis and vulnerability in atheroma of hyperlipidaemic rabbits. $J$ Mol Cell Cardiol 37 969-978.

Li WH, Tanimura M, Luo CC, Datta S, and Chan L (1988) The apolipoprotein multigene family: biosynthesis, structure, structure-function relationships, and evolution. J Lipid Res 29:245-271.
Li X, Feng S, Luo Y, Long K, Lin Z, Ma J, Jiang A, Jin L, Tang Q, Li M, et al. (2018b) Expression profiles of microRNAs in oxidized low-density lipoprotein-stimulated RAW 264.7 cells. In Vitro Cell Dev Biol Anim 54:99-110

Li X, Ji Z, Li S, Sun YN, Liu J, Liu Y, Tian W, Zhou YT, and Shang XM (2015b) miR-146a-5p antagonized AGEs- and P.g-LPS-induced ABCA1 and ABCG1 dysregulation in macrophages via IRAK-1 downregulation. Inflammation 38: $1761-1768$

Li X, Zhou Y, Yu C, Yang H, Zhang C, Ye Y, and Xiao S (2015c) Paeonol suppresses lipid accumulation in macrophages via upregulation of the ATP-binding cassette transporter A1 and downregulation of the cluster of differentiation 36. Int J Oncol 46:764-774.

Li XH, Li Y, Cheng ZY, Cai XG, and Wang HM (2015d) The effects of Phellinus linteus polysaccharide extracts on cholesterol efflux in oxidized low-density lipoprotein-loaded THP-1 macrophages. J Investig Med 63:752-757.

Li XM, Tang WH, Mosior MK, Huang Y, Wu Y, Matter W, Gao V, Schmitt D, Didonato JA, Fisher EA, et al. (2013) Paradoxical association of enhanced cholesterol efflux with increased incident cardiovascular risks. Arterioscler Thromb Vasc Biol 33:1696-1705

Li Y, Feng T, Liu P, Liu C, Wang X, Li D, Li N, Chen M, Xu Y, and Si S (2014) Optimization of rutaecarpine as ABCA1 up-regulator for treating atherosclerosis. ACS Med Chem Lett 5:884-888.

Li Y, Shen S, Ding S, and Wang L (2018c) LincRNA DYN-LRB2-2 upregulates cholesterol efflux by decreasing TLR2 expression in macrophages. J Cell Biochem 119: 1911-1921

Li YH, Wang L, Hong B, Xu YN, Si SY, Jiang JD, and Song DQ (2010) Synthesis and structure-activity relationship of 13-hexylberberine analogues as CD36 antagonists. Yao Xue Xue Bao 45:1128-1133.

Li ZM, Xu SW, and Liu PQ (2018d) Salvia miltiorrhizaBurge (Danshen): a golden herbal medicine in cardiovascular therapeutics. Acta Pharmacol Sin 39:802-824. Liang SJ, Zeng DY, Mai XY, Shang JY, Wu QQ, Yuan JN, Yu BX, Zhou P, Zhang FR, Liu YY, et al. (2016) Inhibition of orai1 store-operated calcium channel prevents foam cell formation and atherosclerosis. Arterioscler Thromb Vasc Biol 36:618-628.

Liang YT, Chen J, Jiao R, Peng C, Zuo Y, Lei L, Liu Y, Wang X, Ma KY, Huang Y, et al. (2015) Cholesterol-lowering activity of sesamin is associated with downregulation on genes of sterol transporters involved in cholesterol absorption. $J$ Agric Food Chem 63:2963-2969.

Libby P (2013) Mechanisms of acute coronary syndromes and their implications for therapy. N Engl J Med 368:2004-2013.

Liehn EA, Ponomariov V, Diaconu R, Streata I, Ioana M, Crespo-Avilan GE, Hernández-Reséndiz S, and Cabrera-Fuentes HA (2018) Apolipoprotein E in cardiovascular diseases: novel aspects of an old-fashioned enigma. Arch Med Res 49 $522-529$.

Lin MC, Ou TT, Chang $\mathrm{CH}$, Chan KC, and Wang CJ (2015a) Protocatechuic acid inhibits oleic acid-induced vascular smooth muscle cell proliferation through activation of AMP-activated protein kinase and cell cycle arrest in G0/G1 phase. $J$ Agric Food Chem 63:235-241.

Lin SJ, Lee IT, Chen YH, Lin FY, Sheu LM, Ku HH, Shiao MS, Chen JW, and Chen YL (2007) Salvianolic acid B attenuates MMP-2 and MMP-9 expression in vivo in apolipoprotein-E-deficient mouse aorta and in vitro in LPS-treated human aortic smooth muscle cells. J Cell Biochem 100:372-384.

Lin W, Liu C, Yang H, Wang W, Ling W, and Wang D (2015b) Chicory, a typical vegetable in Mediterranean diet, exerts a therapeutic role in established atherosclerosis in apolipoprotein E-deficient mice. Mol Nutr Food Res 59:1803-1813.

Lin XL, Hu HJ, Liu YB, Hu XM, Fan XJ, Zou WW, Pan YQ, Zhou WQ, Peng MW, and $\mathrm{Gu} \mathrm{CH}$ (2017) Allicin induces the upregulation of ABCA1 expression via PPAR $\gamma / \mathrm{LXR} \alpha$ signaling in THP-1 macrophage-derived foam cells. Int $J$ Mol Med 39:1452-1460.

Lin XL, Liu MH, Hu HJ, Feng HR, Fan XJ, Zou WW, Pan YQ, Hu XM, and Wang Z (2015c) Curcumin enhanced cholesterol efflux by upregulating ABCA1 expression through AMPK-SIRT1-LXR $\alpha$ signaling in THP-1 macrophage-derived foam cells. DNA Cell Biol 34:561-572.

Linton MRF, Yancey PG, Davies SS, Jerome WG, Linton EF, Song WL, Doran AC, and Vickers KC (2000) The role of lipids and lipoproteins in atherosclerosis, in Endotext (Feingold KR, Anawalt B, Boyce A, Chrousos G, Dungan K, Grossman A Hershman JM, Kaltsas G, Koch C, Kopp P, et al., eds), MDText.com, Inc., South Dartmouth, MA

Liu B, Liu NN, Liu WH, Zhang SW, Zhang JZ, Li AQ, and Liu SM (2016a) Inhibition of lectin-like oxidized low-density lipoprotein receptor-1 reduces cardiac fibroblast proliferation by suppressing GATA binding protein 4. Biochem Biophys Res Commun 475:329-334.

Liu C, Wang W, Lin W, Ling W, and Wang D (2016b) Established atherosclerosis might be a prerequisite for chicory and its constituent protocatechuic acid to promote endothelium-dependent vasodilation in mice. Mol Nutr Food Res 60 $2141-2150$

Liu DS, Wang SL, Li JM, Liang ES, Yan MZ, and Gao W (2017a) Allicin improves carotid artery intima-media thickness in coronary artery disease patients with hyperhomocysteinemia. Exp Ther Med 14:1722-1726.

Liu J, Huang GQ, and Ke ZP (2019) Silence of long intergenic noncoding RNA HOTAIR ameliorates oxidative stress and inflammation response in ox-LDL treated human macrophages by upregulating miR-330-5p. J Cell Physiol 234: $5134-5142$

Liu K, Zhao W, Gao X, Huang F, Kou J, and Liu B (2012a) Diosgenin ameliorates palmitate-induced endothelial dysfunction and insulin resistance via blocking IKK $\beta$ and IRS-1 pathways. Atherosclerosis 223:350-358.

Liu LT, Guo G, Wu M, and Zhang WG (2012b) The progress of the research on cardiovascular effects and acting mechanism of polydatin. Chin J Integr Med 18:714-719. Liu N, Wu C, Sun L, Zheng J, and Guo P (2014a) Sesamin enhances cholesterol efflux in RAW264.7 macrophages. Molecules 19:7516-7527.

Liu R, Li J, Cheng Y, Huo T, Xue J, Liu Y, Liu J, and Chen X (2015) Effects of ellagic acid-rich extract of pomegranates peel on regulation of cholesterol metabolism and its molecular mechanism in hamsters. Food Funct 6:780-787. 
Liu W, He P, Cheng B, Mei CL, Wang YF, and Wan JJ (2010a) Chlamydia pneumoniae disturbs cholesterol homeostasis in human THP-1 macrophages via JNKPPAR $y$ dependent signal transduction pathways. Microbes Infect 12:1226-1235.

Liu WH, Lin CC, Wang ZH, Mong MC, and Yin MC (2010b) Effects of protocatechuic acid on trans fat induced hepatic steatosis in mice. $J$ Agric Food Chem 58: 10247-10252.

Liu X, Tang Y, Cui Y, Zhang H, and Zhang D (2016c) Autophagy is associated with cell fate in the process of macrophage-derived foam cells formation and progress. J Biomed Sci 23:57.

Liu Y and Tang C (2012) Regulation of ABCA1 functions by signaling pathways. Biochim Biophys Acta 1821:522-529.

Liu Y, Zhong Y, Chen H, Wang D, Wang M, Ou JS, and Xia M (2017b) Retinol-binding protein-dependent cholesterol uptake regulates macrophage foam cell formation and promotes atherosclerosis. Circulation 135:1339-1354

Liu YR, Chen JJ, and Dai M (2014b) Paeonol protects rat vascular endothelial cells from ox-LDL-induced injury in vitro via downregulating microRNA-21 expression and TNF- $\alpha$ release. Acta Pharmacol Sin 35:483-488.

Liu Z, Wang J, Huang E, Gao S, Li H, Lu J, Tian K, Little PJ, Shen X, Xu S, et al. (2014c) Tanshinone IIA suppresses cholesterol accumulation in human macrophages: role of heme oxygenase-1. J Lipid Res 55:201-213.

Liu ZM, Ho SC, Chen YM, Tang N, and Woo J (2014d) Effect of whole soy and purified isoflavone daidzein on renal function-a 6-month randomized controlled trial in equol-producing postmenopausal women with prehypertension. Clin Biochem $\mathbf{4 7}$ $1250-1256$

Liu ZM, Ho SC, Woo J, Chen YM, and Wong C (2014e) Randomized controlled trial of whole soy and isoflavone daidzein on menopausal symptoms in equol-producing Chinese postmenopausal women. Menopause 21:653-660.

Li-Weber M (2009) New therapeutic aspects of flavones: the anticancer properties of Scutellaria and its main active constituents wogonin, baicalein and baicalin. Cancer Treat Rev 35:57-68.

Llaverias G, Lacasa D, Vázquez-Carrera M, Sánchez RM, Laguna JC, and Alegret M (2005) Cholesterol regulation of genes involved in sterol trafficking in human THP1 macrophages. Mol Cell Biochem 273:185-191.

Lopez D and McLean MP (1999) Sterol regulatory element-binding protein-1a binds to cis elements in the promoter of the rat high density lipoprotein receptor SR-BI gene. Endocrinology 140:5669-5681.

Low H, Hoang A, and Sviridov D (2012) Cholesterol efflux assay. $J$ Vis Exp 61:3810

Lu L, Liu H, Peng J, Gan L, Shen L, Zhang Q, Li L, Zhang L, Su C, and Jiang Y (2010) Regulations of the key mediators in inflammation and atherosclerosis by aspirin in human macrophages. Lipids Health Dis 9:16.

Luciani MF, Denizot F, Savary S, Mattei MG, and Chimini G (1994) Cloning of two novel ABC transporters mapping on human chromosome 9. Genomics 21:150-159.

Lukasova M, Malaval C, Gille A, Kero J, and Offermanns S (2011) Nicotinic acid inhibits progression of atherosclerosis in mice through its receptor GPR109A expressed by immune cells. J Clin Invest 121:1163-1173.

Lund-Katz S and Phillips MC (2010) High density lipoprotein structure-function and role in reverse cholesterol transport. Subcell Biochem 51:183-227.

Luo X, Fang S, Xiao Y, Song F, Zou T, Wang M, Xia M, and Ling W (2012) Cyanidin-3glucoside suppresses TNF- $\alpha$-induced cell proliferation through the repression of Nox activator 1 in mouse vascular smooth muscle cells: involvement of the STAT3 signaling. Mol Cell Biochem 362:211-218.

Luo Y, Duan H, Qian Y, Feng L, Wu Z, Wang F, Feng J, Yang D, Qin Z, and Yan X (2017) Macrophagic CD146 promotes foam cell formation and retention during atherosclerosis. Cell Res 27:352-372.

Lusis AJ (2000) Atherosclerosis. Nature 407:233-241.

Lv O, Wang L, Li J, Ma Q, and Zhao W (2016) Effects of pomegranate peel polyphenols on lipid accumulation and cholesterol metabolic transformation in L-02 human hepatic cells via the PPAR y-ABCA1/CYP7A1 pathway. Food Funct 7:4976-4983.

Lv YC, Yang J, Yao F, Xie W, Tang YY, Ouyang XP, He PP, Tan YL, Li L, Zhang M, et al. (2015) Diosgenin inhibits atherosclerosis via suppressing the MiR-19b-induced downregulation of ATP-binding cassette transporter A1. Atherosclerosis $\mathbf{2 4 0}$ $80-89$.

Lyssenko NN, Hata M, Dhanasekaran P, Nickel M, Nguyen D, Chetty PS, Saito H, Lund-Katz S, and Phillips MC (2012) Influence of C-terminal $\alpha$-helix hydrophobicity and aromatic amino acid content on apolipoprotein A-I functionality. Biochim Biophys Acta 1821:456-463.

Ma Y, Gong X, Mo Y, and Wu S (2016) Polydatin inhibits the oxidative stress-induced proliferation of vascular smooth muscle cells by activating the eNOS/SIRT1 pathway. Int $J$ Mol Med 37:1652-1660.

Maganto-Garcia E, Tarrio M, and Lichtman AH (2012) Mouse models of atherosclerosis. Curr Protoc Immunol Chapter 15:Unit 15.24.1-23.

Maguire EM, Pearce SWA, and Xiao Q (2019) Foam cell formation: a new target for fighting atherosclerosis and cardiovascular disease. Vascul Pharmacol 112:54-71.

Mahley RW (1988) Apolipoprotein E: cholesterol transport protein with expanding role in cell biology. Science 240:622-630.

Majdalawieh AF and Ro H-S (2014) The anti-atherogenic properties of sesamin are mediated via improved macrophage cholesterol efflux through PPAR $\gamma 1-\mathrm{LXR} \alpha$ and MAPK signaling. Int $J$ Vitam Nutr Res 84:79-91.

Majdalawieh AF and Ro HS (2015) Sesamol and sesame (Sesamum indicum) oil enhance macrophage cholesterol efflux via up-regulation of PPAR $\gamma 1$ and LXR $\alpha$ transcriptional activity in a MAPK-dependent manner. Eur J Nutr 54:691-700.

Majka SM, Miller HL, Helm KM, Acosta AS, Childs CR, Kong R, and Klemm DJ (2014) Analysis and isolation of adipocytes by flow cytometry, in Methods in Enzymology (Macdougald OA ed) pp 281-296, Academic Press, London.

Mäkinen PI, Lappalainen JP, Heinonen SE, Leppänen P, Lähteenvuo MT, Aarnio JV, Heikkilä J, Turunen MP, and Ylä-Herttuala S (2010) Silencing of either SR-A or CD36 reduces atherosclerosis in hyperlipidaemic mice and reveals reciprocal upregulation of these receptors. Cardiovasc Res 88:530-538.

Makino T, Hishida A, Goda Y, and Mizukami H (2008) Comparison of the major flavonoid content of $\mathrm{S}$. baicalensis, S. lateriflora, and their commercial products. J Nat Med 62:294-299.
Malekpour-Dehkordi Z, Javadi E, Doosti M, Paknejad M, Nourbakhsh M, Yassa N Gerayesh-Nejad S, and Heshmat R (2013) S-Allylcysteine, a garlic compound, increases ABCA1 expression in human THP-1 macrophages. Phytother Res 27: 357-361.

Malerød L, Juvet LK, Hanssen-Bauer A, Eskild W, and Berg T (2002) Oxysterolactivated LXRalpha/RXR induces hSR-BI-promoter activity in hepatoma cells and preadipocytes. Biochem Biophys Res Commun 299:916-923.

Malerød L, Sporstøl M, Juvet LK, Mousavi SA, Gjøen T, Berg T, Roos N, and Eskild W (2005) Bile acids reduce SR-BI expression in hepatocytes by a pathway involving FXR/RXR, SHP, and LRH-1. Biochem Biophys Res Commun 336:1096-1105.

Manna PR, Sennoune SR, Martinez-Zaguilan R, Slominski AT, and Pruitt K (2015) Regulation of retinoid mediated cholesterol efflux involves liver $\mathrm{X}$ receptor activation in mouse macrophages. Biochem Biophys Res Commun 464:312-317.

Manning-Tobin JJ, Moore KJ, Seimon TA, Bell SA, Sharuk M, Alvarez-Leite JI, de Winther MP, Tabas I, and Freeman MW (2009) Loss of SR-A and CD36 activity reduces atherosclerotic lesion complexity without abrogating foam cell formation in hyperlipidemic mice. Arterioscler Thromb Vasc Biol 29:19-26.

Mao M, Lei H, Liu Q, Chen Y, Zhao L, Li Q, Luo S, Zuo Z, He Q, Huang W, et al. (2014) Effects of miR-33a-5P on ABCA1/G1-mediated cholesterol efflux under inflammatory stress in THP-1 macrophages. PLoS One 9:e109722.

McCarthy C, Duffy MM, Mooney D, James WG, Griffin MD, Fitzgerald DJ, and Belton $\mathrm{O}$ (2013) IL-10 mediates the immunoregulatory response in conjugated linoleic acid-induced regression of atherosclerosis. FASEB J 27:499-510.

McGrath KC, Li XH, Puranik R, Liong EC, Tan JT, Dy VM, DiBartolo BA, Barter PJ, Rye KA, and Heather AK (2009) Role of 3beta-hydroxysteroid-delta 24 reductase in mediating antiinflammatory effects of high-density lipoproteins in endothelial cells. Arterioscler Thromb Vasc Biol 29:877-882.

McLaren JE, Michael DR, Ashlin TG, and Ramji DP (2011a) Cytokines, macrophage lipid metabolism and foam cells: implications for cardiovascular disease therapy. Prog Lipid Res 50:331-347.

McLaren JE, Michael DR, Guschina IA, Harwood JL, and Ramji DP (2011b) Eicosapentaenoic acid and docosahexaenoic acid regulate modified LDL uptake and macropinocytosis in human macrophages. Lipids 46:1053-1061.

McLure KG, Gesner EM, Tsujikawa L, Kharenko OA, Attwell S, Campeau E, Wasiak S, Stein A, White A, Fontano E, et al. (2013) RVX-208, an inducer of ApoA-I in humans, is a BET bromodomain antagonist. PLoS One 8:e83190.

McNeish J, Aiello RJ, Guyot D, Turi T, Gabel C, Aldinger C, Hoppe KL, Roach ML, Royer LJ, de Wet J, et al. (2000) High density lipoprotein deficiency and foam cell accumulation in mice with targeted disruption of ATP-binding cassette transporter-1. Proc Natl Acad Sci USA 97:4245-4250.

Mehta JL, Sanada N, Hu CP, Chen J, Dandapat A, Sugawara F, Satoh H, Inoue K, Kawase Y, Jishage K, et al. (2007) Deletion of LOX-1 reduces atherogenesis in LDLR knockout mice fed high cholesterol diet. Circ Res 100:1634-1642.

Meir KS and Leitersdorf E (2004) Atherosclerosis in the apolipoprotein-E-deficient mouse: a decade of progress. Arterioscler Thromb Vasc Biol 24:1006-1014.

Meuwese MC, de Groot E, Duivenvoorden R, Trip MD, Ose L, Maritz FJ, Basart DC, Kastelein JJ, Habib R, Davidson MH, et al.; CAPTIVATE Investigators (2009) ACAT inhibition and progression of carotid atherosclerosis in patients with familial hypercholesterolemia: the CAPTIVATE randomized trial. JAMA 301: 1131-1139.

Meyer JM, Graf GA, and van der Westhuyzen DR (2013) New developments in selective cholesteryl ester uptake. Curr Opin Lipidol 24:386-392.

Meyers CD, Kamanna VS, and Kashyap ML (2004) Niacin therapy in atherosclerosis. Curr Opin Lipidol 15:659-665.

Michael Gibson C, Korjian S, Tricoci P, Daaboul Y, Yee M, Jain P, Alexander JH, Steg PG, Lincoff AM, Kastelein JJ, et al. (2016) Safety and tolerability of CSL112, a reconstituted, infusible, plasma-derived apolipoprotein A-I, after acute myocardial infarction: the AEGIS-I trial (ApoA-I event reducing in ischemic syndromes I). Circulation 134:1918-1930.

Mietus-Snyder M, Glass CK, and Pitas RE (1998) Transcriptional activation of scavenger receptor expression in human smooth muscle cells requires AP-1/c-Jun and C/EBPbeta: both AP-1 binding and JNK activation are induced by phorbol esters and oxidative stress. Arterioscler Thromb Vasc Biol 18:1440-1449.

Millar JS and Cuchel M (2015) ApoA-I-directed therapies for the management of atherosclerosis. Curr Atheroscler Rep 17:60.

Min KJ, Um HJ, Cho KH, and Kwon TK (2013) Curcumin inhibits oxLDL-induced CD36 expression and foam cell formation through the inhibition of p38 MAPK phosphorylation. Food Chem Toxicol 58:77-85.

Miyazaki A, Sakashita N, Lee O, Takahashi K, Horiuchi S, Hakamata H, Morganelli PM, Chang CC, and Chang TY (1998) Expression of ACAT-1 protein in human atherosclerotic lesions and cultured human monocytes-macrophages. Arterioscler Thromb Vasc Biol 18:1568-1574.

Momtazi AA, Banach M, Pirro M, Katsiki N, and Sahebkar A (2017) Regulation of PCSK9 by nutraceuticals. Pharmacol Res 120:157-169.

Mooney D, McCarthy C, and Belton O (2012) Effects of conjugated linoleic acid isomers on monocyte, macrophage and foam cell phenotype in atherosclerosis. Pros taglandins Other Lipid Mediat 98:56-62.

Moore KJ, Kunjathoor VV, Koehn SL, Manning JJ, Tseng AA, Silver JM, McKee M, and Freeman MW (2005) Loss of receptor-mediated lipid uptake via scavenger receptor A or CD36 pathways does not ameliorate atherosclerosis in hyperlipidemic mice. J Clin Invest 115:2192-2201.

Moore KJ, Sheedy FJ, and Fisher EA (2013) Macrophages in atherosclerosis: a dynamic balance. Nat Rev Immunol 13:709-721.

Moore RE, Kawashiri MA, Kitajima K, Secreto A, Millar JS, Pratico D, and Rader DJ (2003) Apolipoprotein A-I deficiency results in markedly increased atherosclerosis in mice lacking the LDL receptor. Arterioscler Thromb Vasc Biol 23:1914-1920.

Morini E, Rizzacasa B, Pucci S, Polidoro C, Ferrè F, Caporossi D, Helmer Citterich M, Novelli G, and Amati F (2016) The human rs1050286 polymorphism alters LOX-1 expression through modifying miR-24 binding. J Cell Mol Med 20:181-187.

Morita M, Hayashi T, Ochiai M, Maeda M, Yamaguchi T, Ina K, and Kuzuya M (2014) Oral supplementation with a combination of L-citrulline and L-arginine 
rapidly increases plasma L-arginine concentration and enhances NO bioavailability. Biochem Biophys Res Commun 454:53-57.

Morris GE, Braund PS, Moore JS, Samani NJ, Codd V, and Webb TR (2017) Coronary artery disease-associated LIPA coding variant rs1051338 reduces lysosomal acid lipase levels and activity in lysosomes. Arterioscler Thromb Vasc Biol 37: $1050-1057$.

Mozos I, Stoian D, Caraba A, Malainer C, Horbańczuk JO, and Atanasov AG (2018) Lycopene and vascular health. Front Pharmacol 9:521.

Mukhamedova N, Escher G, D’Souza W, Tchoua U, Grant A, Krozowski Z, Bukrinsky M, and Sviridov D (2008) Enhancing apolipoprotein A-I-dependent cholesterol ef flux elevates cholesterol export from macrophages in vivo. J Lipid Res 49 $2312-2322$.

Murao K, Yu X, Imachi H, Cao WM, Chen K, Matsumoto K, Nishiuchi T, Wong NC, and Ishida T (2008) Hyperglycemia suppresses hepatic scavenger receptor class B type I expression. Am J Physiol Endocrinol Metab 294:E78-E87.

Murata M, Peränen J, Schreiner R, Wieland F, Kurzchalia TV, and Simons K (1995) VIP21/caveolin is a cholesterol-binding protein. Proc Natl Acad Sci USA 92 10339-10343.

Murphy AJ, Akhtari M, Tolani S, Pagler T, Bijl N, Kuo CL, Wang M, Sanson M, Abramowicz S, Welch C, et al. (2011) ApoE regulates hematopoietic stem cell proliferation, monocytosis, and monocyte accumulation in atherosclerotic lesions in mice. J Clin Invest 121:4138-4149.

Nafisa A, Gray SG, Cao Y, Wang T, Xu S, Wattoo FH, Barras M, Cohen N, Kamato D, and Little PJ (2018) Endothelial function and dysfunction: impact of metformin. Pharmacol Ther 192:150-162.

Nagashima M, Watanabe T, Terasaki M, Tomoyasu M, Nohtomi K, Kim-Kaneyama J, Miyazaki A, and Hirano T (2011) Native incretins prevent the development of atherosclerotic lesions in apolipoprotein E knockout mice. Diabetologia 54: 2649-2659.

Nagelin MH, Srinivasan S, Nadler JL, and Hedrick CC (2009) Murine 12/15-lipoxygenase regulates ATP-binding cassette transporter G1 protein degradation through p38- and JNK2-dependent pathways. J Biol Chem 284:31303-31314.

Nagy L, Tontonoz P, Alvarez JG, Chen H, and Evans RM (1998) Oxidized LDL regulates macrophage gene expression through ligand activation of PPARgamma. Cell 93:229-240.

Nakamura T, Shibata N, Nishimoto-Shibata T, Feng D, Ikemoto M, Motojima K, IsoO N, Tsukamoto K, Tsujimoto M, and Arai H (2005) Regulation of SR-BI protein levels by phosphorylation of its associated protein, PDZK1. Proc Natl Acad Sci USA 102:13404-13409.

Nakaya K, Ayaori M, and Ikewaki K (2017) Role of ATP-binding cassette transporters A1 and G1 in reverse cholesterol transport and atherosclerosis, in The HDL Handbook, 3rd ed (Komoda T ed) pp 121-151, Academic Press, London.

Napolitano M, De Pascale C, Wheeler-Jones C, Botham KM, and Bravo E (2007) Effects of lycopene on the induction of foam cell formation by modified LDL. Am $J$ Physiol Endocrinol Metab 293:E1820-E1827.

Narasimhulu CA, Riad A, and Parthasarathy S (2018) Sesame oil and an aqueous extract derived from sesame oil enhance regression of preexisting atherosclerotic lesions in low-density lipoprotein receptor knockout mice. J Med Food 21:641-646.

Nicholls SJ, Andrews J, Kastelein JJP, Merkely B, Nissen SE, Ray KK, Schwartz GG, Worthley SG, Keyserling C, Dasseux JL, et al. (2018) Effect of serial infusions of CER-001, a pre- $\beta$ high-density lipoprotein mimetic, on coronary atherosclerosis in patients following acute coronary syndromes in the CER-001 atherosclerosis regression acute coronary syndrome trial: a randomized clinical trial. JAMA Cardiol 3:815-822.

Nicholls SJ, Gordon A, Johansson J, Wolski K, Ballantyne CM, Kastelein JJP, Taylor A, Borgman M, and Nissen SE (2011) Efficacy and safety of a novel oral inducer of apolipoprotein a-I synthesis in statin-treated patients with stable coronary artery disease a randomized controlled trial. J Am Coll Cardiol 57:1111-1119.

Nicholls SJ, Tuzcu EM, Sipahi I, Schoenhagen P, Crowe T, Kapadia S, and Nissen SE (2006) Relationship between atheroma regression and change in lumen size after infusion of apolipoprotein A-I Milano. J Am Coll Cardiol 47:992-997.

Nicolosi RJ, Wilson TA, and Krause BR (1998) The ACAT inhibitor, CI-1011 is effective in the prevention and regression of aortic fatty streak area in hamsters. Atherosclerosis 137:77-85.

Nie XQ, Chen HH, Zhang JY, Zhang YJ, Yang JW, Pan HJ, Song WX, Murad F, He YQ, and Bian K (2016) Rutaecarpine ameliorates hyperlipidemia and hyperglycemia in fat-fed, streptozotocin-treated rats via regulating the IRS-1/PI3K/Akt and AMPK/ACC2 signaling pathways. Acta Pharmacol Sin 37:483-496.

Niesor EJ, Schwartz GG, Perez A, Stauffer A, Durrwell A, Bucklar-Suchankova G, Benghozi R, Abt M, and Kallend D (2015) Statin-induced decrease in ATP-binding cassette transporter A1 expression via microRNA33 induction may counteract cholesterol efflux to high-density lipoprotein. Cardiovasc Drugs Ther 29:7-14.

Nissen SE, Nicholls SJ, Wolski K, Howey DC, McErlean E, Wang MD, Gomez EV, and Russo JM (2007) Effects of a potent and selective PPAR-alpha agonist in patients with atherogenic dyslipidemia or hypercholesterolemia: two randomized controlled trials. JAMA 297:1362-1373.

Nissen SE, Tsunoda T, Tuzcu EM, Schoenhagen P, Cooper CJ, Yasin M, Eaton GM, Lauer MA, Sheldon WS, Grines CL, et al. (2003) Effect of recombinant ApoA-I Milano on coronary atherosclerosis in patients with acute coronary syndromes: a randomized controlled trial. JAMA 290:2292-2300.

Nissen SE, Tuzcu EM, Brewer HB, Sipahi I, Nicholls SJ, Ganz P, Schoenhagen P, Waters DD, Pepine CJ, Crowe TD, et al.; ACAT Intravascular Atherosclerosis Treatment Evaluation (ACTIVATE) Investigators (2006) Effect of ACAT inhibition on the progression of coronary atherosclerosis. N Engl J Med 354:1253-1263.

Niu N, Xu S, Xu Y, Little PJ, and Jin ZG (2019) Targeting mechanosensitive transcription factors in atherosclerosis. Trends Pharmacol Sci 40:253-266.

Nizamutdinova IT, Oh HM, Min YN, Park SH, Lee MJ, Kim JS, Yean MH, Kang SS, Kim YS, Chang KC, et al. (2007) Paeonol suppresses intercellular adhesion molecule-1 expression in tumor necrosis factor-alpha-stimulated human umbilical vein endothelial cells by blocking p38, ERK and nuclear factor-kappaB signaling pathways. Int Immunopharmacol 7:343-350.
Nofer JR, Kehrel B, Fobker M, Levkau B, Assmann G, and von Eckardstein A (2002) HDL and arteriosclerosis: beyond reverse cholesterol transport. Atherosclerosis 161:1-16.

Oche B, Chen L, Ma YK, Yang Y, Li CX, Geng X, Qiu LZ, Gao XM, and Wang H (2016) Cryptotanshinone and wogonin up-regulate eNOS in vascular endothelial cells via ER $\alpha$ and down-regulate iNOS in LPS stimulated vascular smooth muscle cells via ER $\beta$. Arch Pharm Res 39:249-258.

Ochiai A, Miyata S, Shimizu M, Inoue J, and Sato R (2015) Piperine induces hepatic low-density lipoprotein receptor expression through proteolytic activation of sterol regulatory element-binding proteins. PLoS One 10:e0139799.

Ogura M, Ayaori M, Terao Y, Hisada T, Iizuka M, Takiguchi S, Uto-Kondo H, Yakushiji E, Nakaya K, Sasaki M, et al. (2011) Proteasomal inhibition promotes ATP-binding cassette transporter A1 (ABCA1) and ABCG1 expression and cholesterol efflux from macrophages in vitro and in vivo. Arterioscler Thromb Vasc Biol 31:1980-1987.

Ogura S, Kakino A, Sato Y, Fujita Y, Iwamoto S, Otsui K, Yoshimoto R, and Sawamura T (2009) Lox-1: the multifunctional receptor underlying cardiovascular dysfunction. Circ J 73:1993-1999.

Oh J, Riek AE, Darwech I, Funai K, Shao J, Chin K, Sierra OL, Carmeliet G, Ostlund RE Jr, and Bernal-Mizrachi C (2015) Deletion of macrophage Vitamin D receptor promotes insulin resistance and monocyte cholesterol transport to accelerate atherosclerosis in mice. Cell Reports 10:1872-1886.

Oh J, Riek AE, Zhang RM, Williams SAS, Darwech I, and Bernal-Mizrachi C (2018) Deletion of JNK2 prevents vitamin-D-deficiency-induced hypertension and atherosclerosis in mice. $J$ Steroid Biochem Mol Biol 177:179-186.

Oh J, Weng S, Felton SK, Bhandare S, Riek A, Butler B, Proctor BM, Petty M, Chen $\mathrm{Z}$, Schechtman KB, et al. (2009) 1,25(OH)2 vitamin d inhibits foam cell formation and suppresses macrophage cholesterol uptake in patients with type 2 diabetes mellitus. Circulation 120:687-698.

Okazaki H, Igarashi M, Nishi M, Sekiya M, Tajima M, Takase S, Takanashi M, Ohta K, Tamura Y, Okazaki S, et al. (2008) Identification of neutral cholesterol ester hydrolase, a key enzyme removing cholesterol from macrophages. J Biol Chem 283 : 33357-33364.

Okazaki H, Osuga J, Tsukamoto K, Isoo N, Kitamine T, Tamura Y, Tomita S, Sekiya M, Yahagi N, Iizuka Y, et al. (2002) Elimination of cholesterol ester from macrophage foam cells by adenovirus-mediated gene transfer of hormone-sensitive lipase. J Biol Chem 277:31893-31899.

Okonkwo TJ, Osadebe PO, and Proksch P (2016) Bioactive phenylpropanoids, phenolic acid and phytosterol from landolphia owariensis $\mathrm{P}$. Beauv stringy seed pulp. Phytother Res 30:78-83.

Olagnier D, Lavergne RA, Meunier E, Lefêvre L, Dardenne C, Aubouy A, BenoitVical F, Ryffel B, Coste A, Berry A, et al. (2011) Nrf2, a PPARy alternative pathway to promote CD36 expression on inflammatory macrophages: implication for malaria. PLoS Pathog 7:e1002254.

Olson JA (1989) Provitamin A function of carotenoids: the conversion of $\beta$-carotene into vitamin A. J Nutr 119:105-108.

O’Rourke L, Gronning LM, Yeaman SJ, and Shepherd PR (2002) Glucose-dependent regulation of cholesterol ester metabolism in macrophages by insulin and leptin. $J$ Biol Chem 277:42557-42562.

Orsó E, Broccardo C, Kaminski WE, Böttcher A, Liebisch G, Drobnik W, Götz A Chambenoit O, Diederich W, Langmann T, et al. (2000) Transport of lipids from golgi to plasma membrane is defective in tangier disease patients and Abc1deficient mice. Nat Genet 24:192-196.

Ostadmohammadi V, Milajerdi A, Ghayour-Mobarhan M, Ferns G, Taghizadeh M, Badehnoosh B, Mirzaei H, and Asemi Z (2019) The effects of vitamin D supplementation on glycemic control, lipid profiles and C-reactive protein among patients with cardiovascular disease: a systematic review and meta-analysis of randomized controlled trials. Curr Pharm Des 25:201-210.

Otocka-Kmiecik A, Mikhailidis DP, Nicholls SJ, Davidson M, Rysz J, and Banach M (2012) Dysfunctional HDL: a novel important diagnostic and therapeutic target in cardiovascular disease? Prog Lipid Res 51:314-324.

Ouimet M, Franklin V, Mak E, Liao X, Tabas I, and Marcel YL (2011) Autophagy regulates cholesterol efflux from macrophage foam cells via lysosomal acid lipase. Cell Metab 13:655-667.

Out R, Hoekstra M, Habets K, Meurs I, de Waard V, Hildebrand RB, Wang Y, Chimini G, Kuiper J, Van Berkel TJ, et al. (2008) Combined deletion of macrophage ABCA1 and ABCG1 leads to massive lipid accumulation in tissue macrophages and distinct atherosclerosis at relatively low plasma cholesterol levels. Arterioscler Thromb Vasc Biol 28:258-264.

Ouyang XP, Zhou SH, Tian SW, Li X, He PP, Yin WL, and Tang CK (2012) Effect of arecoline on cholesterol efflux and ATP-binding cassette transporter A1 expression in RAW 264.7 macrophage-derived foam cells. Chin J Arteriosclerosis 4:180-185

Paigen B, Morrow A, Holmes PA, Mitchell D, and Williams RA (1987) Quantitative assessment of atherosclerotic lesions in mice. Atherosclerosis 68:231-240.

Palozza P, Catalano A, Simone RE, Mele MC, and Cittadini A (2012) Effect of lycopene and tomato products on cholesterol metabolism. Ann Nutr Metab 61:126-134 Palozza P, Simone R, Catalano A, Parrone N, Monego G, and Ranelletti FO (2011) Lycopene regulation of cholesterol synthesis and efflux in human macrophages. $J$ Nutr Biochem 22:971-978.

Pan LL and Dai M (2009) Paeonol from Paeonia suffruticosa prevents TNF-alphainduced monocytic cell adhesion to rat aortic endothelial cells by suppression of VCAM-1 expression. Phytomedicine 16:1027-1032.

Pandey NR, Renwick J, Misquith A, Sokoll K, and Sparks DL (2008) Linoleic acidenriched phospholipids act through peroxisome proliferator-activated receptors alpha to stimulate hepatic apolipoprotein A-I secretion. Biochemistry 47:1579-1587.

Panousis CG, Evans G, and Zuckerman SH (2001) TGF-beta increases cholesterol efflux and ABC-1 expression in macrophage-derived foam cells: opposing the effects of IFN-gamma. J Lipid Res 42:856-863.

Panousis CG and Zuckerman SH (2000a) Interferon-gamma induces downregulation of Tangier disease gene (ATP-binding-cassette transporter 1) in macrophagederived foam cells. Arterioscler Thromb Vasc Biol 20:1565-1571. 
Panousis CG and Zuckerman SH (2000b) Regulation of cholesterol distribution in macrophage-derived foam cells by interferon-gamma. J Lipid Res 41:75-83.

Park DW, Baek K, Kim JR, Lee JJ, Ryu SH, Chin BR, and Baek SH (2009a) Resveratrol inhibits foam cell formation via NADPH oxidase 1- mediated reactive oxygen species and monocyte chemotactic protein-1. Exp Mol Med 41:171-179.

Park JH, Noh SK, and Kim CS (2010) Effects of paprika (Capsicum annuum L.) on serum lipid profile and -tocopherol concentration in rats fed a high-cholesterol diet. Korean J Food \& Nutr 23:311-317.

Park SH, Kim JL, Lee ES, Han SY, Gong JH, Kang MK, and Kang YH (2011) Dietary ellagic acid attenuates oxidized LDL uptake and stimulates cholesterol efflux in murine macrophages. J Nutr 141:1931-1937.

Park SH, Paek JH, Shin D, Lee JY, Lim SS, and Kang YH (2015) Purple perilla extracts with $\alpha$-asarone enhance cholesterol efflux from oxidized LDL-exposed macrophages. Int J Mol Med 35:957-965.

Park YM (2014) CD36, a scavenger receptor implicated in atherosclerosis. Exp Mol Med 46:e99.

Park YM, Febbraio M, and Silverstein RL (2009b) CD36 modulates migration of mouse and human macrophages in response to oxidized LDL and may contribute to macrophage trapping in the arterial intima. J Clin Invest 119:136-145.

Parthasarathy S, Santanam N, Ramachandran S, and Meilhac O (1999) Oxidants and antioxidants in atherogenesis. An appraisal. J Lipid Res 40:2143-2157.

Patti AM, Al-Rasadi K, Giglio RV, Nikolic D, Mannina C, Castellino G, Chianetta R, Banach M, Cicero AFG, Lippi G, et al. (2018) Natural approaches in metabolic syndrome management. Arch Med Sci 14:422-441.

Penson P, Long DL, Howard G, Howard VJ, Jones SR, Martin SS, Mikhailidis DP, Muntner P, Rizzo M, Rader DJ, et al. (2019) Associations between cardiovascular disease, cancer, and very low high-density lipoprotein cholesterol in the REasons for Geographical and Racial Differences in Stroke (REGARDS) study. Cardiovasc Res 115:204-212.

Penson P, McGowan M, and Banach M (2017) Evaluating bempedoic acid for the treatment of hyperlipidaemia. Expert Opin Investig Drugs 26:251-259.

Perrey S, Legendre C, Matsuura A, Guffroy C, Binet J, Ohbayashi S, Tanaka T, Ortuno JC, Matsukura T, Laugel T, et al. (2001) Preferential pharmacological inhibition of macrophage ACAT increases plaque formation in mouse and rabbit models of atherogenesis. Atherosclerosis 155:359-370.

Peterson J, Dwyer J, Adlercreutz H, Scalbert A, Jacques P, and McCullough ML (2010) Dietary lignans: physiology and potential for cardiovascular disease risk reduction. Nutr Rev 68:571-603.

Petri MH, Laguna-Fernandez A, Arnardottir H, Wheelock CE, Perretti M, Hansson GK, and Bäck M (2017) Aspirin-triggered lipoxin A4 inhibits atherosclerosis progression in apolipoprotein $\mathrm{E}^{-/-}$mice. Br J Pharmacol 174:4043-4054.

Phillips MC (2013) New insights into the determination of HDL structure by apolipoproteins: thematic review series: high density lipoprotein structure, function, and metabolism. J Lipid Res 54:2034-2048.

Phillips MC (2014) Molecular mechanisms of cellular cholesterol efflux. J Biol Chem 289:24020-24029.

Phillips MC (2018) Is ABCA1 a lipid transfer protein? J Lipid Res 59:749-763.

Picaud S, Wells C, Felletar I, Brotherton D, Martin S, Savitsky P, Diez-Dacal B, Philpott M, Bountra C, Lingard H, et al. (2013) RVX-208, an inhibitor of BET transcriptional regulators with selectivity for the second bromodomain. Proc Natl Acad Sci USA 110:19754-19759.

Pietsch A, Weber C, Goretzki M, Weber PC, and Lorenz RL (1995) N-3 but not N-6 fatty acids reduce the expression of the combined adhesion and scavenger receptor CD36 in human monocytic cells. Cell Biochem Funct 13:211-216.

Pinkosky SL, Newton RS, Day EA, Ford RJ, Lhotak S, Austin RC, Birch CM, Smith BK, Filippov S, Groot PHE, et al. (2016) Liver-specific ATP-citrate lyase inhibition by bempedoic acid decreases LDL-C and attenuates atherosclerosis. Nat Commun 7:13457.

Plump AS, Smith JD, Hayek T, Aalto-Setälä K, Walsh A, Verstuyft JG, Rubin EM, and Breslow JL (1992) Severe hypercholesterolemia and atherosclerosis in apolipoprotein E-deficient mice created by homologous recombination in ES cells. Cell 71:343-353.

Qian H, Zhao X, Cao P, Lei J, Yan N, and Gong X (2017) Structure of the human lipid exporter ABCA1. Cell 169:1228-1239.e10

Qiao L, Zhang X, Liu M, Liu X, Dong M, Cheng J, Zhang X, Zhai C, Song Y, Lu H, et al. (2017) Ginsenoside Rb1 enhances atherosclerotic plaque stability by improving autophagy and lipid metabolism in macrophage foam cells. Front Pharmacol 8:727.

Qin L, Yang YB, Yang YX, Zhu N, Liu Z, Ni YG, Li SX, Zheng XL, and Liao DF (2016) Inhibition of macrophage-derived foam cell formation by ezetimibe via the caveolin-1/MAPK pathway. Clin Exp Pharmacol Physiol 43:182-192.

Qin S, Huang L, Gong J, Shen S, Huang J, Ren H, and Hu H (2017) Efficacy and safety of turmeric and curcumin in lowering blood lipid levels in patients with cardiovascular risk factors: a meta-analysis of randomized controlled trials. Nutr $J$ 16:68.

Qin Y, Xia M, Ma J, Hao Y, Liu J, Mou H, Cao L, and Ling W (2009) Anthocyanin supplementation improves serum LDL- and HDL-cholesterol concentrations associated with the inhibition of cholesteryl ester transfer protein in dyslipidemic subjects. Am J Clin Nutr 90:485-492.

Quinn MT, Parthasarathy S, Fong LG, and Steinberg D (1987) Oxidatively modified low density lipoproteins: a potential role in recruitment and retention of monocyte/ macrophages during atherogenesis. Proc Natl Acad Sci USA 84:2995-2998.

Rafatian N, Milne RW, Leenen FH, and Whitman SC (2013) Role of reninangiotensin system in activation of macrophages by modified lipoproteins. Am $J$ Physiol Heart Circ Physiol 305:H1309-H1320.

Raffai RL (2012) Apolipoprotein E regulation of myeloid cell plasticity in atherosclerosis. Curr Opin Lipidol 23:471-478.

Raghavan S, Singh NK, Gali S, Mani AM, and Rao GN (2018) Protein kinase C $\theta$ via activating transcription factor 2-mediated CD36 expression and foam cell formation of Ly6C ${ }^{\text {hi }}$ cells contributes to atherosclerosis. Circulation 138:2395-2412.

Rahaman SO, Lennon DJ, Febbraio M, Podrez EA, Hazen SL, and Silverstein RL (2006) A CD36-dependent signaling cascade is necessary for macrophage foam cell formation. Cell Metab 4:211-221.
Ramachandran S, Vinitha A, and Kartha CC (2016) Cyclophilin A enhances macrophage differentiation and lipid uptake in high glucose conditions: a cellular mechanism for accelerated macro vascular disease in diabetes mellitus. Cardiovasc Diabetol 15:152.

Ramirez CM, Dávalos A, Goedeke L, Salerno AG, Warrier N, Cirera-Salinas D, Suárez Y, and Fernández-Hernando C (2011) MicroRNA-758 regulates cholesterol efflux through posttranscriptional repression of ATP-binding cassette transporter A1. Arterioscler Thromb Vasc Biol 31:2707-2714.

Ramirez Bosca A, Carrión Gutierrez MA, Soler A, Puerta C, Diez A, Quintanilla E, Bernd A, and Miquel J (1997) Effects of the antioxidant turmeric on lipoprotein peroxides: implications for the prevention of atherosclerosis. Age (Omaha) 20: $165-168$.

Ramírez-Tortosa MC, Mesa MD, Aguilera MC, Quiles JL, Baró L, Ramirez-Tortosa CL, Martinez-Victoria E, and Gil A (1999) Oral administration of a turmeric extract inhibits LDL oxidation and has hypocholesterolemic effects in rabbits with experimental atherosclerosis. Atherosclerosis 147:371-378.

Rao AV and Rao LG (2007) Carotenoids and human health. Pharmacol Res 55: $207-216$.

Rauf A, Imran M, Khan IA, Ur-Rehman M, Gilani SA, Mehmood Z, and Mubarak MS (2018) Anticancer potential of quercetin: a comprehensive review. Phytother Res 32:2109-2130.

Ray KK, Bays HE, Catapano AL, Lalwani ND, Bloedon LT, Sterling LR, Robinson PL, and Ballantyne CM; CLEAR Harmony Trial (2019) Safety and efficacy of bempedoic acid to reduce LDL cholesterol. N Engl J Med 380:1022-1032.

Rayner KJ, Suárez Y, Dávalos A, Parathath S, Fitzgerald ML, Tamehiro N, Fisher EA, Moore KJ, and Fernández-Hernando C (2010) MiR-33 contributes to the regulation of cholesterol homeostasis. Science 328:1570-1573.

Reardon CA, Blachowicz L, White T, Cabana V, Wang Y, Lukens J, Bluestone J, and Getz GS (2001) Effect of immune deficiency on lipoproteins and atherosclerosis in male apolipoprotein E-deficient mice. Arterioscler Thromb Vasc Biol 21: 1011-1016.

Reisinger U, Schwaiger S, Zeller I, Messner B, Stigler R, Wiedemann D, Mayr T, Seger C, Schachner T, Dirsch VM, et al. (2009) Leoligin, the major lignan from Edelweiss, inhibits intimal hyperplasia of venous bypass grafts. Cardiovasc Res $\mathbf{8 2}$ : 542-549.

Relevy NZ, Harats D, Harari A, Ben-Amotz A, Bitzur R, Rühl R, and Shaish A (2015) Vitamin A-deficient diet accelerated atherogenesis in apolipoprotein E(-/-) mice and dietary $\beta$-carotene prevents this consequence. BioMed Res Int 2015:758723.

Remaley AT, Schumacher UK, Stonik JA, Farsi BD, Nazih H, and Brewer HB Jr (1997) Decreased reverse cholesterol transport from Tangier disease fibroblasts. Acceptor specificity and effect of brefeldin on lipid efflux. Arterioscler Thromb Vasc Biol 17:1813-1821.

Ren H, Hao J, Liu T, Zhang D, Lv H, Song E, and Zhu C (2016) Hesperetin suppresses inflammatory responses in lipopolysaccharide-induced RAW 264.7 cells via the inhibition of $\mathrm{NF}-\mathrm{\kappa B}$ and activation of $\mathrm{Nrf} 2 / \mathrm{HO}-1$ pathways. Inflammation 39: 964-973.

Reza JZ, Doosti M, Salehipour M, Packnejad M, Mojarrad M, and Heidari M (2009) Modulation peroxisome proliferators activated receptor alpha (PPAR alpha) and acyl coenzyme A: cholesterol acyltransferase1 (ACAT1) gene expression by fatty acids in foam cell [published correction appears in Lipids Health Dis (2009) 8:47]. Lipids Health Dis 8:38.

Rezvan A, Ni CW, Alberts-Grill N, and Jo H (2011) Animal, in vitro, and ex vivo models of flow-dependent atherosclerosis: role of oxidative stress. Antioxid Redox Signal 15:1433-1448.

Riccioni G, D’Orazio N, Salvatore C, Franceschelli S, Pesce M, and Speranza L (2012) Carotenoids and vitamins $\mathrm{C}$ and $\mathrm{E}$ in the prevention of cardiovascular disease. Int $J$ Vitam Nutr Res 82:15-26.

Ridker PM, Everett BM, Thuren T, MacFadyen JG, Chang WH, Ballantyne C, Fonseca F, Nicolau J, Koenig W, Anker SD, et al.; CANTOS Trial Group (2017) Antiinflammatory therapy with canakinumab for atherosclerotic disease. $N$ Engl $J$ Med 377:1119-1131.

Rigotti A, Miettinen HE, and Krieger M (2003) The role of the high-density lipoprotein receptor SR-BI in the lipid metabolism of endocrine and other tissues. Endocr Rev 24:357-387.

Ringseis R, König B, Leuner B, Schubert S, Nass N, Stangl G, and Eder K (2006) LDL receptor gene transcription is selectively induced by t10c12-CLA but not by c9t11 CLA in the human hepatoma cell line HepG2. Biochim Biophys Acta 1761: $1235-1243$

Ringseis R, Wen G, Saal D, and Eder K (2008) Conjugated linoleic acid isomers reduce cholesterol accumulation in acetylated LDL-induced mouse RAW264.7 macrophage-derived foam cells. Lipids 43:913-923.

Rinne P, Rami M, Nuutinen S, Santovito D, van der Vorst EPC, Guillamat-Prats R, Lyytikäinen LP, Raitoharju E, Oksala N, Ring L, et al. (2017) Melanocortin 1 receptor signaling regulates cholesterol transport in macrophages. Circulation 136 : 83-97.

Robb EL and Stuart JA (2014) Multiple phytoestrogens inhibit cell growth and confer cytoprotection by inducing manganese superoxide dismutase expression. Phytother Res 28:120-131.

Robbins CS, Hilgendorf I, Weber GF, Theurl I, Iwamoto Y, Figueiredo JL, Gorbatov R, Sukhova GK, Gerhardt LM, Smyth D, et al. (2013) Local proliferation dominates lesional macrophage accumulation in atherosclerosis. Nat Med 19: 1166-1172.

Robson J (2008) Lipid modification: cardiovascular risk assessment and the modification of blood lipids for the primary and secondary prevention of cardiovascular disease. Heart 94:1331-1332.

Rodriguez de Sotillo DV and Hadley M (2002) Chlorogenic acid modifies plasma and liver concentrations of: cholesterol, triacylglycerol, and minerals in (fa/fa) Zucker rats. J Nutr Biochem 13:717-726.

Rodríguez-Páez L, Juárez-Sanchez M, Antúnez-Solís J, Baeza I, and Wong C (2003) Alpha-asarone inhibits HMG-CoA reductase, lowers serum LDL-cholesterol levels and reduces biliary CSI in hypercholesterolemic rats. Phytomedicine 10:397-404. 
Rogi T, Tomimori N, Ono Y, and Kiso Y (2011) The mechanism underlying the synergetic hypocholesterolemic effect of sesamin and $\alpha$-tocopherol in rats fed a high-cholesterol diet. J Pharmacol Sci 115:408-416.

Rohatgi A, Khera A, Berry JD, Givens EG, Ayers CR, Wedin KE, Neeland IJ, Yuhanna IS, Rader DR, de Lemos JA, et al. (2014) HDL cholesterol efflux capacity and incident cardiovascular events. N Engl J Med 371:2383-2393.

Rohrer L, Ohnsorg PM, Lehner M, Landolt F, Rinninger F, and von Eckardstein A (2009) High-density lipoprotein transport through aortic endothelial cells involves scavenger receptor BI and ATP-binding cassette transporter G1. Circ Res 104 $1142-1150$.

Rong JX, Blachford C, Feig JE, Bander I, Mayne J, Kusunoki J, Miller C, Davis M, Wilson M, Dehn S, et al. (2013) ACAT inhibition reduces the progression of preexisting, advanced atherosclerotic mouse lesions without plaque or systemic toxicity. Arterioscler Thromb Vasc Biol 33:4-12.

Rong JX, Kusunoki J, Oelkers P, Sturley SL, and Fisher EA (2005) Acyl-coenzymeA (CoA):cholesterol acyltransferase inhibition in rat and human aortic smooth muscle cells is nontoxic and retards foam cell formation. Arterioscler Thromb Vasc Biol 25:122-127.

Rong JX, Shapiro M, Trogan E, and Fisher EA (2003) Transdifferentiation of mouse aortic smooth muscle cells to a macrophage-like state after cholesterol loading. Proc Natl Acad Sci USA 100:13531-13536.

Roosbeek S, Peelman F, Verhee A, Labeur C, Caster H, Lensink MF, Cirulli C, Grooten J, Cochet C, Vandekerckhove J, et al. (2004) Phosphorylation by protein kinase CK2 modulates the activity of the ATP binding cassette A1 transporter. $J$ Biol Chem 279:37779-37788.

Rosendahl AH, Perks CM, Zeng L, Markkula A, Simonsson M, Rose C, Ingvar C, Holly JM, and Jernstrom H (2015) Caffeine and caffeic acid inhibit growth and modify estrogen receptor and insulin-like growth factor I receptor levels in human breast cancer. Clin Cancer Res 21:1877-1887.

Rosenson RS, Brewer HB Jr, Chapman MJ, Fazio S, Hussain MM, Kontush A Krauss RM, Otvos JD, Remaley AT, and Schaefer EJ (2011) HDL measures, particle heterogeneity, proposed nomenclature, and relation to atherosclerotic cardiovascular events. Clin Chem 57:392-410.

Rosenson RS, Brewer HB Jr, Davidson WS, Fayad ZA, Fuster V, Goldstein J, Hellerstein M, Jiang XC, Phillips MC, Rader DJ, et al. (2012) Cholesterol efflux and atheroprotection: advancing the concept of reverse cholesterol transport. Circulation 125:1905-1919.

Ross R (1999) Atherosclerosis-an inflammatory disease. N Engl J Med 340:115-126.

Rothblat GH, de la Llera-Moya M, Atger V, Kellner-Weibel G, Williams DL, and Phillips MC (1999) Cell cholesterol efflux: integration of old and new observations provides new insights. J Lipid Res 40:781-796.

Rothblat GH and Phillips MC (2010) High-density lipoprotein heterogeneity and function in reverse cholesterol transport. Curr Opin Lipidol 21:229-238.

Rubic T, Trottmann M, and Lorenz RL (2004) Stimulation of CD36 and the key effector of reverse cholesterol transport ATP-binding cassette A1 in monocytoid cells by niacin. Biochem Pharmacol 67:411-419.

Rudel LL, Lee RG, and Parini P (2005) ACAT2 is a target for treatment of coronary heart disease associated with hypercholesterolemia. Arterioscler Thromb Vasc Biol 25:1112-1118

Ruiz E, Del Rio M, Somoza B, Ganado P, Sanz M, and Tejerina T (1999) L-Citrulline, the by-product of nitric oxide synthesis, decreases vascular smooth muscle cell proliferation. J Pharmacol Exp Ther 290:310-313.

Ruscica M, Banach M, Sahebkar A, Corsini A, and Sirtori CR (2019) ETC-1002 (Bempedoic acid) for the management of hyperlipidemia: from preclinical studies to phase 3 trials. Expert Opin Pharmacother 20:791-803.

Rust S, Rosier M, Funke H, Real J, Amoura Z, Piette J-C, Deleuze J-F, Brewer HB, Duverger $N$, Denèfle $P$, et al. (1999) Tangier disease is caused by mutations in the gene encoding ATP-binding cassette transporter 1. Nat Genet 22:352-355.

Sabol SL, Brewer HB Jr, and Santamarina-Fojo S (2005) The human ABCG1 gene: identification of LXR response elements that modulate expression in macrophages and liver. J Lipid Res 46:2151-2167.

Sahebkar A, Serban C, Ursoniu S, and Banach M (2016a) Effect of garlic on plasma lipoprotein(a) concentrations: a systematic review and meta-analysis of randomized controlled clinical trials. Nutrition 32:33-40.

Sahebkar A, Serban MC, Gluba-Brzózka A, Mikhailidis DP, Cicero AF, Rysz J, and Banach M (2016b) Lipid-modifying effects of nutraceuticals: an evidence-based approach. Nutrition 32:1179-1192.

Saito I, Saito H, Tamura Y, and Yoshida S (1992) Eicosapentaenoic acid inhibits cholesteryl ester accumulation in rat peritoneal macrophages by decreasing the number of specific binding sites of acetyl LDL. Clin Biochem 25:351-355.

Sakaguchi H, Takeya M, Suzuki H, Hakamata H, Kodama T, Horiuchi S, Gordon S, van der Laan LJ, Kraal G, Ishibashi S, et al. (1998) Role of macrophage scavenger receptors in diet-induced atherosclerosis in mice. Lab Invest 78:423-434.

Sakai K, Igarashi M, Yamamuro D, Ohshiro T, Nagashima S, Takahashi M, Enkhtuvshin B, Sekiya M, Okazaki H, Osuga J, et al. (2014) Critical role of neutral cholesteryl ester hydrolase 1 in cholesteryl ester hydrolysis in murine macrophages. J Lipid Res 55:2033-2040.

Sakashita N, Miyazaki A, Chang CC, Chang TY, Kiyota E, Satoh M, Komohara Y, Morganelli PM, Horiuchi S, and Takeya M (2003) Acyl-coenzyme A:cholesterol acyltransferase 2 (ACAT2) is induced in monocyte-derived macrophages: in vivo and in vitro studies. Lab Invest 83:1569-1581.

Saleheen D, Scott R, Javad S, Zhao W, Rodrigues A, Picataggi A, Lukmanova D, Mucksavage ML, Luben R, Billheimer J, et al. (2015) Association of HDL cholesterol efflux capacity with incident coronary heart disease events: a prospective case-control study. Lancet Diabetes Endocrinol 3:507-513.

Sallam T, Jones M, Thomas BJ, Wu X, Gilliland T, Qian K, Eskin A, Casero D, Zhang Z, Sandhu J, et al. (2018) Transcriptional regulation of macrophage cholesterol efflux and atherogenesis by a long noncoding RNA. Nat Med 24:304-312.

Samsoondar JP, Burke AC, Sutherland BG, Telford DE, Sawyez CG, Edwards JY, Pinkosky SL, Newton RS, and Huff MW (2017) Prevention of diet-induced metabolic dysregulation, inflammation, and atherosclerosis in $\mathrm{Ldll}^{-1-}$ mice by treatment with the ATP-citrate lyase inhibitor bempedoic acid. Arterioscler Thromb Vasc Biol 37:647-656.

Sankaranarayanan S, Kellner-Weibel G, de la Llera-Moya M, Phillips MC, Asztalos $\mathrm{BF}$, Bittman R, and Rothblat GH (2011) A sensitive assay for ABCA1-mediated cholesterol efflux using BODIPY-cholesterol. J Lipid Res 52:2332-2340.

Sano O, Ito S, Kato R, Shimizu Y, Kobayashi A, Kimura Y, Kioka N, Hanada K, Ueda $\mathrm{K}$, and Matsuo M (2014) ABCA1, ABCG1, and ABCG4 are distributed to distinct membrane meso-domains and disturb detergent-resistant domains on the plasma membrane. PLoS One 9:e109886.

Santamarina-Fojo S, Peterson K, Knapper C, Qiu Y, Freeman L, Cheng JF, Osorio J, Remaley A, Yang XP, Haudenschild C, et al. (2000) Complete genomic sequence of the human ABCA1 gene: analysis of the human and mouse ATP-binding cassette A promoter. Proc Natl Acad Sci USA 97:7987-7992.

Sarris J, McIntyre E, and Camfield DA (2013) Plant-based medicines for anxiety disorders, part 2: a review of clinical studies with supporting preclinical evidence [published correction appears in CNS Drugs (2013) 27:675]. CNS Drugs 27:301-319.

Sarver AL, Li L, and Subramanian S (2010) MicroRNA miR-183 functions as an oncogene by targeting the transcription factor EGR1 and promoting tumor cell migration. Cancer Res 70:9570-9580.

Sato Y, Itagaki S, Kurokawa T, Ogura J, Kobayashi M, Hirano T, Sugawara M, and Iseki K (2011) In vitro and in vivo antioxidant properties of chlorogenic acid and caffeic acid. Int $J$ Pharm 403:136-138

Sawamura T, Kume N, Aoyama T, Moriwaki H, Hoshikawa H, Aiba Y, Tanaka T, Miwa S, Katsura Y, Kita T, et al. (1997) An endothelial receptor for oxidized lowdensity lipoprotein. Nature 386:73-77.

Schachter M (2005) Chemical, pharmacokinetic and pharmacodynamic properties of statins: an update. Fundam Clin Pharmacol 19:117-125.

Schaeffer DF, Riazy M, Parhar KS, Chen JH, Duronio V, Sawamura T, and Steinbrecher UP (2009) LOX-1 augments oxLDL uptake by lysoPC-stimulated murine macrophages but is not required for oxLDL clearance from plasma. J Lipid Res 50:1676-1684.

Scharinger B, Messner B, Türkcan A, Schuster D, Vuorinen A, Pitterl F, Heinz K, Arnhard K, Laufer G, Grimm M, et al. (2016) Leoligin, the major lignan from Edelweiss, inhibits 3-hydroxy-3-methyl-glutaryl-CoA reductase and reduces cholesterol levels in ApoE-/- mice. J Mol Cell Cardiol 99:35-46.

Schlager S, Vujic N, Korbelius M, Duta-Mare M, Dorow J, Leopold C, Rainer S, Wegscheider M, Reicher H, Ceglarek U, et al. (2017) Lysosomal lipid hydrolysis provides substrates for lipid mediator synthesis in murine macrophages. Oncotarget 8:40037-40051.

Schlüter KD, Wolf A, Weber M, Schreckenberg R, and Schulz R (2017) Oxidized lowdensity lipoprotein (oxLDL) affects load-free cell shortening of cardiomyocytes in a proprotein convertase subtilisin/kexin 9 (PCSK9)-dependent way. Basic Res Cardiol 112:63.

Schmitz G and Langmann T (2005) Transcriptional regulatory networks in lipid metabolism control ABCA1 expression. Biochim Biophys Acta 1735:1-19.

Schmitz G, Langmann T, and Heimerl S (2001) Role of ABCG1 and other ABCG family members in lipid metabolism. J Lipid Res 42:1513-1520.

Schrader C, Ernst IM, Sinnecker H, Soukup ST, Kulling SE, and Rimbach G (2012) Genistein as a potential inducer of the anti-atherogenic enzyme paraoxonase-1: studies in cultured hepatocytes in vitro and in rat liver in vivo. J Cell Mol Med 16 2331-2341.

Schrijvers DM, De Meyer GR, Herman AG, and Martinet W (2007) Phagocytosis in atherosclerosis: molecular mechanisms and implications for plaque progression and stability. Cardiovasc Res 73:470-480

Schwartz GG, Olsson AG, Abt M, Ballantyne CM, Barter PJ, Brumm J, Chaitman BR, Holme IM, Kallend D, Leiter LA, et al; dal-OUTCOMES Investigators (2012) Effects of dalcetrapib in patients with a recent acute coronary syndrome. $N$ Engl $J$ Med 367:2089-2099.

Schwartz GG, Olsson AG, Ballantyne CM, Barter PJ, Holme IM, Kallend D, Leiter LA, Leitersdorf E, McMurray JJ, Shah PK, et al.; dal-OUTCOMES Committees and Investigators (2009) Rationale and design of the dal-OUTCOMES trial: efficacy and safety of dalcetrapib in patients with recent acute coronary syndrome. Am Heart $J$ 158:896-901.e3.

Schwartz K, Lawn RM, and Wade DP (2000) ABC1 gene expression and ApoA-Imediated cholesterol efflux are regulated by LXR. Biochem Biophys Res Commun 274:794-802.

Segrest JP, Jones MK, De Loof H, Brouillette CG, Venkatachalapathi YV, and Anantharamaiah GM (1992) The amphipathic helix in the exchangeable apolipoproteins: a review of secondary structure and function. J Lipid Res $\mathbf{3 3}$ 141-166.

Seidah NG, Benjannet S, Wickham L, Marcinkiewicz J, Jasmin SB, Stifani S, Basak A, Prat A, and Chretien M (2003) The secretory proprotein convertase neural apoptosis-regulated convertase 1 (NARC-1): liver regeneration and neuronal differentiation. Proc Natl Acad Sci USA 100:928-933.

Sekiya M, Osuga J, Nagashima S, Ohshiro T, Igarashi M, Okazaki H, Takahashi M, Tazoe F, Wada T, Ohta K, et al. (2009) Ablation of neutral cholesterol ester hydrolase 1 accelerates atherosclerosis. Cell Metab 10:219-228.

Sekiya M, Yamamuro D, Ohshiro T, Honda A, Takahashi M, Kumagai M, Sakai K, Nagashima S, Tomoda H, Igarashi M, et al. (2014) Absence of Nceh1 augments 25hydroxycholesterol-induced ER stress and apoptosis in macrophages. J Lipid Res 55:2082-2092.

Selmer D, Senekowitsch-Schmidtke R, Schneider W, and Elstner EF (1997) Binding and uptake of $125 i o d i n e-l a b e l l e d$, oxidized low density lipoprotein by macrophages: comparison of the effects of alpha-tocopherol, probucol, pyridoxal-5' -phosphate and magnesium-pyridoxal-5'-phosphate-glutamate. Z Naturforsch C 52:97-104.

Sendl A, Schliack M, Löser R, Stanislaus F, and Wagner H (1992) Inhibition of cholesterol synthesis in vitro by extracts and isolated compounds prepared from garlic and wild garlic. Atherosclerosis 94:79-85.

Sengupta B, Narasimhulu CA, and Parthasarathy S (2013) Novel technique for generating macrophage foam cells for in vitro reverse cholesterol transport studies. J Lipid Res 54:3358-3372. 
Serban C, Sahebkar A, Ursoniu S, Mikhailidis DP, Rizzo M, Lip GY, Kees Hovingh G, Kastelein JJ, Kalinowski L, Rysz J, et al. (2015) A systematic review and metaanalysis of the effect of statins on plasma asymmetric dimethylarginine concentrations. Sci Rep 5:9902.

Serban MC, Sahebkar A, Dragan S, Stoichescu-Hogea G, Ursoniu S, Andrica F, and Banach M (2016) A systematic review and meta-analysis of the impact of Spirulina supplementation on plasma lipid concentrations. Clin Nutr 35:842-851.

Seres L, Cserepes J, Elkind NB, Törocsik D, Nagy L, Sarkadi B, and Homolya L (2008) Functional ABCG1 expression induces apoptosis in macrophages and other cell types. Biochim Biophys Acta 1778:2378-2387.

Serfaty-Lacrosniere C, Civeira F, Lanzberg A, Isaia P, Berg J, Janus ED, Smith MP Jr, Pritchard PH, Frohlich J, Lees RS, et al. (1994) Homozygous Tangier disease and cardiovascular disease. Atherosclerosis 107:85-98.

Sesso HD, Buring JE, Norkus EP, and Gaziano JM (2005) Plasma lycopene, other carotenoids, and retinol and the risk of cardiovascular disease in men. Am J Clin Nutr 81:990-997.

Shaish A, Harari A, Hananshvili L, Cohen H, Bitzur R, Luvish T, Ulman E, Golan M, Ben-Amotz A, Gavish D, et al. (2006) 9-cis beta-carotene-rich powder of the alga Dunaliella bardawil increases plasma HDL-cholesterol in fibrate-treated patients. Atherosclerosis 189:215-221.

Shankman LS, Gomez D, Cherepanova OA, Salmon M, Alencar GF, Haskins RM, Swiatlowska P, Newman AA, Greene ES, Straub AC, et al. (2015) KLF4-dependent phenotypic modulation of smooth muscle cells has a key role in atherosclerotic plaque pathogenesis. Nat Med 21:628-637.

Sharifiyan F, Movahedian-Attar A, Nili N, and Asgary S (2016) Study of pomegranate (Punica granatum L.) peel extract containing anthocyanins on fatty streak formation in the renal arteries in hypercholesterolemic rabbits. Adv Biomed Res 5:8.

Sharma G, She ZG, Valenta DT, Stallcup WB, and Smith JW (2010) Targeting of macrophage foam cells in atherosclerotic plaque using oligonucleotidefunctionalized nanoparticles. Nano Life 1:207-214.

Sharpe LJ, Rao G, Jones PM, Glancey E, Aleidi SM, George AM, Brown AJ, and Gelissen IC (2015) Cholesterol sensing by the ABCG1 lipid transporter: requirement of a CRAC motif in the final transmembrane domain. Biochim Biophys Acta 1851:956-964.

She S, Liu W, Li T, and Hong Y (2014) Effects of puerarin in STZ-induced diabetic rats by oxidative stress and the TGF- $\beta 1 /$ Smad2 pathway. Food Funct 5:944-950.

Shen WJ, Asthana S, Kraemer FB, and Azhar S (2018a) Scavenger receptor B type 1: expression, molecular regulation, and cholesterol transport function. J Lipid Res 59:1114-1131.

Shen WJ, Azhar S, and Kraemer FB (2018b) SR-B1: a unique multifunctional receptor for cholesterol influx and efflux. Annu Rev Physiol 80:95-116.

Shetty S, Eckhardt ER, Post SR, and van der Westhuyzen DR (2006) Phosphatidylinositol-3-kinase regulates scavenger receptor class B type I subcellular localization and selective lipid uptake in hepatocytes. Arterioscler Thromb Vasc Biol 26: $2125-2131$

Shi H, Zhang C, Pasupuleti V, Hu X, Prosdocimo DA, Wu W, Qing Y, Wu S, Mohammad H, Gerson SL, et al. (2017) CCN3 regulates macrophage foam cell formation and atherosclerosis. Am J Pathol 187:1230-1237.

Shi L, Fan PS, Fang JX, and Han ZX (1988) Inhibitory effects of paeonol on experimental atherosclerosis and platelet aggregation of rabbit. Zhongguo Yao Li Xue Bao 9:555-558.

Shi Y, Hu J, Geng J, Hu T, Wang B, Yan W, Jiang Y, Li J, and Liu S (2018) Berberine treatment reduces atherosclerosis by mediating gut microbiota in apoE-/- mice. Biomed Pharmacother 107:1556-1563.

Shibuya K, Kawamine K, Miura T, Ozaki C, Edano T, Mizuno K, Yoshinaka Y, and Tsunenari Y (2018) Design, synthesis and pharmacology of aortic-selective acyl-CoA: cholesterol O-acyltransferase (ACAT/SOAT) inhibitors. Bioorg Med Chem 26:4001-4013.

Shimaoka T, Kume N, Minami M, Hayashida K, Kataoka H, Kita T, and Yonehara S (2000) Molecular cloning of a novel scavenger receptor for oxidized low density lipoprotein, SR-PSOX, on macrophages. J Biol Chem 275:40663-40666.

Shiu SW, Tan KC, Wong Y, Leng L, and Bucala R (2009) Glycoxidized LDL increases lectin-like oxidized low density lipoprotein receptor-1 in diabetes mellitus. Atherosclerosis 203:522-527.

Shoji N, Umeyama A, Takemoto T, Kajiwara A, and Ohizumi Y (1986) Isolation of evodiamine, a powerful cardiotonic principle, from Evodia rutaecarpa Bentham (Rutaceae). J Pharm Sci 75:612-613.

Simental-Mendía LE and Guerrero-Romero F (2019) Effect of resveratrol supplementation on lipid profile in subjects with dyslipidemia: a randomized doubleblind, placebo-controlled trial. Nutrition 58:7-10.

Singaraja RR, Bocher V, James ER, Clee SM, Zhang LH, Leavitt BR, Tan B, BrooksWilson A, Kwok A, Bissada N, et al. (2001) Human ABCA1 BAC transgenic mice show increased high density lipoprotein cholesterol and ApoAI-dependent efflux stimulated by an internal promoter containing liver $\mathrm{X}$ receptor response elements in intron 1. J Biol Chem 276:33969-33979.

Singh JP, Kauffman R, Bensch W, Wang GM, McClelland P, Bean J, Montrose C, Mantlo N, and Wagle A (2005) Identification of a novel selective peroxisome proliferator-activated receptor alpha agonist, 2-methyl-2-(4-\{3-[1-(4-methylbenzyl)5-oxo-4,5-dihydro-1H-1,2,4-triazol-3-yl]propyl\}phenoxy)propanoic acid (LY518674), that produces marked changes in serum lipids and apolipoprotein A-1 expression. Mol Pharmacol 68:763-768.

Singh V, Tiwari RL, Dikshit M, and Barthwal MK (2009) Models to study atherosclerosis: a mechanistic insight. Curr Vasc Pharmacol 7:75-109.

Sini S, Deepa D, Harikrishnan S, and Jayakumari N (2017) High-density lipoprotein from subjects with coronary artery disease promotes macrophage foam cell formation: role of scavenger receptor CD36 and ERK/MAPK signaling. Mol Cell Biochem 427:23-34.

Skottová N and Krecman V (1998) Silymarin as a potential hypocholesterolaemic drug. Physiol Res 47:1-7.

Smith SC Jr, Allen J, Blair SN, Bonow RO, Brass LM, Fonarow GC, Grundy SM, Hiratzka L, Jones D, Krumholz HM, et al.; AHA/ACC; National Heart, Lung, and
Blood Institute (2006) AHA/ACC guidelines for secondary prevention for patients with coronary and other atherosclerotic vascular disease: 2006 update: endorsed by the National Heart, Lung, and Blood Institute. Circulation 113:2363-2372.

Smithies O and Maeda N (1995) Gene targeting approaches to complex genetic diseases: atherosclerosis and essential hypertension. Proc Natl Acad Sci USA 92: $5266-5272$.

Soltani B, Bodaghabadi N, Ghaemi N, and Sadeghizadeh M (2017) Radiation-induced surge of macrophage foam cell formation, oxidative damage, and cytokine release is attenuated by a nanoformulation of curcumin. Int J Radiat Biol 93:303-314.

Son IS, Kim JH, Sohn HY, Son KH, Kim JS, and Kwon CS (2007) Antioxidative and hypolipidemic effects of diosgenin, a steroidal saponin of yam (Dioscorea spp.), on high-cholesterol fed rats. Biosci Biotechnol Biochem 71:3063-3071.

Song GJ, Kim SM, Park KH, Kim J, Choi I, and Cho KH (2015) SR-BI mediates high density lipoprotein (HDL)-induced anti-inflammatory effect in macrophages. Biochem Biophys Res Commun 457:112-118.

Song Y, Zhang LJ, Li H, Gu Y, Li FF, Jiang LN, Liu F, Ye J, and Li Q (2013) Polyunsaturated fatty acid relatively decreases cholesterol content in THP-1 macrophage-derived foam cell: partly correlates with expression profile of CIDE and PAT members. Lipids Health Dis 12:111.

Sosić-Jurjević B, Filipović B, Ajdzanović V, Brkić D, Ristić N, Stojanoski MM, Nestorović N, Trifunović S, and Sekulić M (2007) Subcutaneously administrated genistein and daidzein decrease serum cholesterol and increase triglyceride levels in male middle-aged rats. Exp Biol Med (Maywood) 232:1222-1227.

Soumian S, Albrecht C, Davies AH, and Gibbs RG (2005) ABCA1 and atherosclerosis. Vasc Med 10:109-119.

Speer T, Owala FO, Holy EW, Zewinger S, Frenzel FL, Stähli BE, Razavi M, Triem S, Cvija H, Rohrer L, et al. (2014) Carbamylated low-density lipoprotein induces endothelial dysfunction. Eur Heart $J$ 35:3021-3032.

Sporstøl M, Tapia G, Malerød L, Mousavi SA, and Berg T (2005) Pregnane X receptor-agonists down-regulate hepatic ATP-binding cassette transporter A1 and scavenger receptor class B type I. Biochem Biophys Res Commun 331:1533-1541.

Stangl H, Graf GA, Yu L, Cao G, and Wyne K (2002) Effect of estrogen on scavenger receptor BI expression in the rat. $J$ Endocrinol 175:663-672.

Stein S and Matter CM (2011) Protective roles of SIRT1 in atherosclerosis. Cell Cycle 10:640-647.

Steinberg D and Witztum JL (2010) Oxidized low-density lipoprotein and atherosclerosis. Arterioscler Thromb Vasc Biol 30:2311-2316.

Stoekenbroek RM, Stroes ES, and Hovingh GK (2015) ApoA-I mimetics, in High Density Lipoproteins: From Biological Understanding to Clinical Exploitation (von Eckardstein A and Kardassis D eds) pp 631-648, Springer International Publishing, Cham, Switzerland.

Straus DS and Glass CK (2007) Anti-inflammatory actions of PPAR ligands: new insights on cellular and molecular mechanisms. Trends Immunol 28:551-558.

Stumpf C, Fan Q, Hintermann C, Raaz D, Kurfürst I, Losert S, Pflederer W, Achenbach S, Daniel WG, and Garlichs CD (2013) Anti-inflammatory effects of danshen on human vascular endothelial cells in culture. Am J Chin Med 41: 1065-1077.

Su KH, Lin SJ, Wei J, Lee KI, Zhao JF, Shyue SK, and Lee TS (2014) The essential role of transient receptor potential vanilloid 1 in simvastatin-induced activation of endothelial nitric oxide synthase and angiogenesis. Acta Physiol (Oxf) 212 191-204.

Su YR, Dove DE, Major AS, Hasty AH, Boone B, Linton MF, and Fazio S (2005) Reduced ABCA1-mediated cholesterol efflux and accelerated atherosclerosis in apolipoprotein E-deficient mice lacking macrophage-derived ACAT1. Circulation 111:2373-2381.

Sugasawa N, Katagi A, Kurobe H, Nakayama T, Nishio C, Takumi H, Higashiguch F, Aihara KI, Shimabukuro M, Sata M, et al. (2019) Inhibition of atherosclerotic plaque development by oral administration of $\alpha$-glucosyl hesperidin and waterdispersible hesperetin in apolipoprotein E knockout mice. J Am Coll Nutr 38: $15-22$.

Suits AG, Chait A, Aviram M, and Heinecke JW (1989) Phagocytosis of aggregated lipoprotein by macrophages: low density lipoprotein receptor-dependent foam-cell formation. Proc Natl Acad Sci USA 86:2713-2717.

Sultan Alvi S, Ansari IA, Khan I, Iqbal J, and Khan MS (2017) Potential role of lycopene in targeting proprotein convertase subtilisin/kexin type-9 to combat hypercholesterolemia. Free Radic Biol Med 108:394-403.

Sun L, Li E, Wang F, Wang T, Qin Z, Niu S, and Qiu C (2015) Quercetin increases macrophage cholesterol efflux to inhibit foam cell formation through activating PPARy-ABCA1 pathway. Int J Clin Exp Pathol 8:10854-10860.

Sung JH, Lee SJ, Park KH, and Moon TW (2004) Isoflavones inhibit 3-hydroxy-3methylglutaryl coenzyme A reductase in vitro. Biosci Biotechnol Biochem 68 428-432.

Suzuki H, Kurihara Y, Takeya M, Kamada N, Kataoka M, Jishage K, Ueda O, Sakaguchi H, Higashi T, Suzuki T, et al. (1997) A role for macrophage scavenger receptors in atherosclerosis and susceptibility to infection. Nature 386:292-296.

Szanto A, Benko S, Szatmari I, Balint BL, Furtos I, Rühl R, Molnar S, Csiba L, Garuti R, Calandra S, et al. (2004) Transcriptional regulation of human CYP27 integrates retinoid, peroxisome proliferator-activated receptor, and liver X receptor signaling in macrophages. Mol Cell Biol 24:8154-8166.

Tabas I, García-Cardeña G, and Owens GK (2015) Recent insights into the cellular biology of atherosclerosis. J Cell Biol 209:13-22.

Tai MC, Tsang SY, Chang LY, and Xue H (2005) Therapeutic potential of wogonin: a naturally occurring flavonoid. CNS Drug Rev 11:141-150.

Tai MH, Chen PK, Chen PY, Wu MJ, Ho CT, and Yen JH (2014) Curcumin enhances cell-surface LDLR level and promotes LDL uptake through downregulation of PCSK9 gene expression in HepG2 cells. Mol Nutr Food Res 58:2133-2145.

Tall AR (1990) Plasma high density lipoproteins. Metabolism and relationship to atherogenesis. J Clin Invest 86:379-384.

Tamminen M, Mottino G, Qiao JH, Breslow JL, and Frank JS (1999) Ultrastructure of early lipid accumulation in ApoE-deficient mice. Arterioscler Thromb Vasc Biol 19:847-853. 
Tanaka M, Koyama M, Dhanasekaran P, Nguyen D, Nickel M, Lund-Katz S, Saito H, and Phillips MC (2008) Influence of tertiary structure domain properties on the functionality of apolipoprotein A-I. Biochemistry 47:2172-2180.

Tanaka M, Matsuo Y, Yamakage H, Masuda S, Terada Y, Muranaka K, Wada H, Hasegawa K, Shimatsu A, and Satoh-Asahara N (2016) Differential effects of GLP1 receptor agonist on foam cell formation in monocytes between non-obese and obese subjects. Metabolism 65:1-11.

Tancevski I, Demetz E, Eller P, Duwensee K, Hoefer J, Heim C, Stanzl U, Wehinger A, Auer K, Karer R, et al. (2010) The liver-selective thyromimetic T-0681 influences reverse cholesterol transport and atherosclerosis development in mice. PLoS One 5:e8722.

Tang B, Du J, Wang J, Tan G, Gao Z, Wang Z, and Wang L (2012) Alpinetin suppresses proliferation of human hepatoma cells by the activation of MKK7 and elevates sensitization to cis-diammined dichloridoplatium. Oncol Rep $\mathbf{2 7}$ $1090-1096$.

Tang FT, Cao Y, Wang TQ, Wang LJ, Guo J, Zhou XS, Xu SW, Liu WH, Liu PQ, and Huang HQ (2011) Tanshinone IIA attenuates atherosclerosis in ApoE(-/-) mice through down-regulation of scavenger receptor expression. Eur J Pharmacol 650: 275-284.

Tao H, Yancey PG, Babaev VR, Blakemore JL, Zhang Y, Ding L, Fazio S, and Linton MF (2015a) Macrophage SR-BI mediates efferocytosis via Src/PI3K/Rac1 signaling and reduces atherosclerotic lesion necrosis. J Lipid Res 56:1449-1460.

Tao J, Liu CZ, Yang J, Xie ZZ, Ma MM, Li XY, Li FY, Wang GL, Zhou JG, Du YH, et al. (2015b) ClC-3 deficiency prevents atherosclerotic lesion development in ApoE-/- mice. J Mol Cell Cardiol 87:237-247.

Tardif JC, Ballantyne CM, Barter P, Dasseux JL, Fayad ZA, Guertin MC, Kastelein JJP, Keyserling C, Klepp H, Koenig W, et al.; Can HDL Infusions Significantly QUicken Atherosclerosis REgression (CHI-SQUARE) Investigators (2014) Effects of the high-density lipoprotein mimetic agent CER-001 on coronary atherosclerosis in patients with acute coronary syndromes: a randomized trial. Eur Heart $J \mathbf{3 5}$ $3277-3286$

Tardif JC, Grégoire J, L'Allier PL, Anderson TJ, Bertrand O, Reeves F, Title LM, Alfonso F, Schampaert E, Hassan A, et al.; Avasimibe and Progression of Lesions on UltraSound (A-PLUS) Investigators (2004) Effects of the acyl coenzyme A:cholesterol acyltransferase inhibitor avasimibe on human atherosclerotic lesions. Circulation 110:3372-3377.

Tardif JC, Grégoire J, L'Allier PL, Ibrahim R, Lespérance J, Heinonen TM, Kouz S, Berry C, Basser R, Lavoie MA, et al.; Effect of rHDL on Atherosclerosis-Safety and Efficacy (ERASE) Investigators (2007) Effects of reconstituted high-density lipoprotein infusions on coronary atherosclerosis: a randomized controlled trial. JAMA 297:1675-1682

Tarling EJ (2013) Expanding roles of ABCG1 and sterol transport. Curr Opin Lipidol 24:138-146.

Tarling EJ and Edwards PA (2011) ATP binding cassette transporter G1 (ABCG1) is an intracellular sterol transporter. Proc Natl Acad Sci USA 108:19719-19724.

Tarling EJ and Edwards PA (2012) Dancing with the sterols: critical roles for ABCG1, ABCA1, miRNAs, and nuclear and cell surface receptors in controlling cellular sterol homeostasis. Biochim Biophys Acta 1821:386-395.

Tarr PT, Tarling EJ, Bojanic DD, Edwards PA, and Baldán A (2009) Emerging new paradigms for ABCG transporters. Biochim Biophys Acta 1791:584-593.

Terasaka N, Miyazaki A, Kasanuki N, Ito K, Ubukata N, Koieyama T, Kitayama K, Tanimoto T, Maeda N, and Inaba T (2007) ACAT inhibitor pactimibe sulfate (CS505) reduces and stabilizes atherosclerotic lesions by cholesterol-lowering and direct effects in apolipoprotein E-deficient mice. Atherosclerosis 190:239-247.

Terpstra V, Kondratenko N, and Steinberg D (1997) Macrophages lacking scavenger receptor A show a decrease in binding and uptake of acetylated low-density lipoprotein and of apoptotic thymocytes, but not of oxidatively damaged red blood cells. Proc Natl Acad Sci USA 94:8127-8131.

Teupser D, Persky AD, and Breslow JL (2003) Induction of atherosclerosis by low-fat, semisynthetic diets in LDL receptor-deficient C57BL/6J and FVB/NJ mice: comparison of lesions of the aortic root, brachiocephalic artery, and whole aorta (en face measurement). Arterioscler Thromb Vasc Biol 23:1907-1913.

Teupser D, Thiery J, Walli AK, and Seidel D (1996) Determination of LDL- and scavenger-receptor activity in adherent and non-adherent cultured cells with a new single-step fluorometric assay. Biochim Biophys Acta 1303:193-198.

Tian K, Ogura S, Little PJ, Xu SW, and Sawamura T (2019a) Targeting LOX-1 in atherosclerosis and vasculopathy: current knowledge and future perspectives. Ann N Y Acad Sci 1443:34-53.

Tian KM, Li JJ, and Xu SW (2019b) Rutaecarpine: a promising cardiovascular protective alkaloid from Evodia rutaecarpa (Wu Zhu Yu). Pharmacol Res 141:541-550.

Tiwari RL, Singh V, and Barthwal MK (2008) Macrophages: an elusive yet emerging therapeutic target of atherosclerosis. Med Res Rev 28:483-544.

Tontonoz P, Nagy L, Alvarez JG, Thomazy VA, and Evans RM (1998) PPARgamma promotes monocyte/macrophage differentiation and uptake of oxidized LDL. Cell 93:241-252.

Tošović J, Marković S, Dimitrić Marković JM, Mojović M, and Milenković D (2017) Antioxidative mechanisms in chlorogenic acid. Food Chem 237:390-398.

Toth PP and Banach M (2019) Statins: then and Now. Methodist DeBakey Cardiovasc $J$ 15:23-31.

Toth PP, Barylski M, Nikolic D, Rizzo M, Montalto G, and Banach M (2014) Should low high-density lipoprotein cholesterol (HDL-C) be treated? Best Pract Res Clin Endocrinol Metab 28:353-368.

Tsuboi T, Maeda M, and Hayashi T (2018) Administration of L-arginine plus L-citrulline or L-citrulline alone successfully retarded endothelial senescence. PLoS One 13:e192252.

Ulrich V, Konaniah ES, Herz J, Gerard RD, Jung E, Yuhanna IS, Ahmed M, Hui DY, Mineo C, and Shaul PW (2014) Genetic variants of ApoE and ApoER2 differentially modulate endothelial function. Proc Natl Acad Sci USA 111:13493-13498.

Um MY, Ahn J, and Ha TY (2013) Hypolipidaemic effects of cyanidin 3-glucoside rich extract from black rice through regulating hepatic lipogenic enzyme activities. J Sci Food Agric 93:3126-3128.
Ursoniu S, Sahebkar A, Serban MC, Antal D, Mikhailidis DP, Cicero A, Athyros V, Rizzo M, Rysz J, and Banach M; Lipid and Blood Pressure Meta-analysis Collaboration Group (2017) Lipid-modifying effects of krill oil in humans: systematic review and meta-analysis of randomized controlled trials. Nutr Rev 75:361-373.

Ussher JR and Drucker DJ (2012) Cardiovascular biology of the incretin system. Endocr Rev 33:187-215.

Uto-Kondo H, Ayaori M, Nakaya K, Takiguchi S, Yakushiji E, Ogura M, Terao Y, Ozasa H, Sasaki M, Komatsu T, et al. (2014) Citrulline increases cholesterol efflux from macrophages in vitro and ex vivo via ATP-binding cassette transporters. $J$ Clin Biochem Nutr 55:32-39.

Uto-Kondo H, Ayaori M, Ogura M, Nakaya K, Ito M, Suzuki A, Takiguchi S, Yakushiji E, Terao Y, Ozasa H, et al. (2010) Coffee consumption enhances high-density lipoprotein-mediated cholesterol efflux in macrophages. Circ Res 106:779-787.

Vaisman BL, Lambert G, Amar M, Joyce C, Ito T, Shamburek RD, Cain WJ, Fruchart-Najib J, Neufeld ED, Remaley AT, et al. (2001) ABCA1 overexpression leads to hyperalphalipoproteinemia and increased biliary cholesterol excretion in transgenic mice. J Clin Invest 108:303-309.

Vaisman BL, Vishnyakova TG, Freeman LA, Amar MJ, Demosky SJ, Liu C, Stonik JA, Sampson ML, Pryor M, Bocharov AV, et al. (2015) Endothelial expression of scavenger receptor class B, type I protects against development of atherosclerosis in mice. BioMed Res Int 2015:607120.

Van Eck M, Bos IS, Hildebrand RB, Van Rij BT, and Van Berkel TJ (2004) Dual role for scavenger receptor class B, type I on bone marrow-derived cells in atherosclerotic lesion development. Am J Pathol 165:785-794.

Vanhoutte PM, Shimokawa H, Feletou M, and Tang EH (2017) Endothelial dysfunction and vascular disease - a 30th anniversary update. Acta Physiol (Oxf) 219: $22-96$

Vasamsetti SB, Karnewar S, Gopoju R, Gollavilli PN, Narra SR, Kumar JM, and Kotamraju S (2016) Resveratrol attenuates monocyte-to-macrophage differentiation and associated inflammation via modulation of intracellular GSH homeostasis: relevance in atherosclerosis. Free Radic Biol Med 96:392-405.

Vengrenyuk Y, Nishi H, Long X, Ouimet M, Savji N, Martinez FO, Cassella CP, Moore KJ, Ramsey SA, Miano JM, et al. (2015) Cholesterol loading reprograms the microRNA-143/145-myocardin axis to convert aortic smooth muscle cells to a dysfunctional macrophage-like phenotype. Arterioscler Thromb Vasc Biol 35:535-546. Venkateswaran A, Repa JJ, Lobaccaro JM, Bronson A, Mangelsdorf DJ, and Edwards PA (2000) Human white/murine ABC8 mRNA levels are highly induced in lipidloaded macrophages. A transcriptional role for specific oxysterols. J Biol Chem $\mathbf{2 7 5}$ : 14700-14707.

Vergeer M, Korporaal SJ, Franssen R, Meurs I, Out R, Hovingh GK, Hoekstra M, Sierts JA, Dallinga-Thie GM, Motazacker MM, et al. (2011) Genetic variant of the scavenger receptor BI in humans. $N$ Engl J Med 364:136-145.

Vickers KC, Palmisano BT, Shoucri BM, Shamburek RD, and Remaley AT (2011) MicroRNAs are transported in plasma and delivered to recipient cells by highdensity lipoproteins. Nat Cell Biol 13:423-433.

Viñals M, Bermúdez I, Llaverias G, Alegret M, Sanchez RM, Vázquez-Carrera M, and Laguna JC (2005) Aspirin increases CD36, SR-BI, and ABCA1 expression in human THP-1 macrophages. Cardiovasc Res 66:141-149.

Voloshyna I, Hai O, Littlefield MJ, Carsons S, and Reiss AB (2013) Resveratrol mediates anti-atherogenic effects on cholesterol flux in human macrophages and endothelium via PPAR $\gamma$ and adenosine. Eur J Pharmacol 698:299-309.

Voloshyna I, Seshadri S, Anwar K, Littlefield MJ, Belilos E, Carsons SE, and Reiss $\mathrm{AB}$ (2014) Infliximab reverses suppression of cholesterol efflux proteins by TNF- $\alpha$ : a possible mechanism for modulation of atherogenesis. Biomed Res Int 2014: 312647.

Vromman A, Ruvkun V, Shvartz E, Wojtkiewicz G, Santos Masson G, Tesmenitsky Y, Folco E, Gram H, Nahrendorf M, Swirski FK, et al. (2019) Stage-dependent differential effects of interleukin-1 isoforms on experimental atherosclerosis. Eur Heart J 40:2482-2491.

Wagner H, Bauer R, Melchart D, Xiao P-G, and Staudinger A (2011) Fructus Evodiae Wuzhuyu, in Chromatographic Fingerprint Analysis of Herbal Medicines: Thinlayer and High Performance Liquid Chromatography of Chinese Drugs (Wagner $\mathrm{H}$, Bauer R, Melchart D, Xiao P-G, and Staudinger A eds) pp 391-401, Springer, Vienna, Austria.

Wågsäter D, Dimberg J, and Sirsjö A (2003) Induction of ATP-binding cassette A1 by all-trans retinoic acid: possible role of liver X receptor-alpha. Int $J$ Mol Med 11: $419-423$

Wallace TC, Slavin M, and Frankenfeld CL (2016) Systematic review of anthocyanins and markers of cardiovascular disease. Nutrients 8:E32.

Waltenberger B, Mocan A, Šmejkal K, Heiss EH, and Atanasov AG (2016) Natural products to counteract the epidemic of cardiovascular and metabolic disorders. Molecules 21:E807.

Wan JJ, Cheng B, Wang YF, Mei CL, Liu W, Ke L, and He P (2009) Ghrelin downregulates ACAT-1 in THP-1 derived foam cells via growth hormone secretagogue receptor-dependent pathway. Zhonghua Xin Xue Guan Bing Za Zhi 37:1030-1034.

Wang B, He PP, Zeng GF, Zhang T, and Ou Yang XP (2017a) miR-467b regulates the cholesterol ester formation via targeting ACAT1 gene in RAW 264.7 macrophages. Biochimie 132:38-44.

Wang C, Xu W, Liang M, Huang D, and Huang K (2019a) CTRP13 inhibits atherosclerosis via autophagy-lysosome-dependent degradation of CD36. FASEB J 33: $2290-2300$

Wang D, Hiebl V, Ladurner A, Latkolik SL, Bucar F, Heiß EH, Dirsch VM, and Atanasov AG (2018a) 6-Dihydroparadol, a ginger constituent, enhances cholesterol efflux from THP-1-derived macrophages. Mol Nutr Food Res 62:e1800011.

Wang D, Özen C, Abu-Reidah IM, Chigurupati S, Patra JK, Horbanczuk JO, Jóźwik A, Tzvetkov NT, Uhrin P, and Atanasov AG (2018b) Vasculoprotective effects of pomegranate (Punica granatum L.). Front Pharmacol 9:544.

Wang D, Tosevska A, Heiß EH, Ladurner A, Mölzer C, Wallner M, Bulmer A, Wagner $\mathrm{KH}$, Dirsch VM, and Atanasov AG (2017b) Bilirubin decreases macrophage cholesterol efflux and ATP-binding cassette transporter A1 protein expression. J Am Heart Assoc 6:e05520. 
Wang D, Wei X, Yan X, Jin T, and Ling W (2010a) Protocatechuic acid, a metabolite of anthocyanins, inhibits monocyte adhesion and reduces atherosclerosis in apolipoprotein E-deficient mice. J Agric Food Chem 58:12722-12728.

Wang D, Xia M, Yan X, Li D, Wang L, Xu Y, Jin T, and Ling W (2012a) Gut microbiota metabolism of anthocyanin promotes reverse cholesterol transport in mice via repressing miRNA-10b. Circ Res 111:967-981.

Wang D, Yan X, Xia M, Yang Y, Li D, Li X, Song F, and Ling W (2014a) Coenzyme Q10 promotes macrophage cholesterol efflux by regulation of the activator protein1/miR-378/ATP-binding cassette transporter G1-signaling pathway. Arterioscler Thromb Vasc Biol 34:1860-1870.

Wang D, Zou T, Yang Y, Yan X, and Ling W (2011) Cyanidin-3-O- $\beta$-glucoside with the aid of its metabolite protocatechuic acid, reduces monocyte infiltration in apolipoprotein E-deficient mice. Biochem Pharmacol 82:713-719.

Wang J, Xu P, Xie X, Li J, Zhang J, Wang J, Hong F, Li J, Zhang Y, Song Y, et al. (2017c) DBZ (Danshensu Bingpian Zhi), a novel natural compound derivative, attenuates atherosclerosis in apolipoprotein E-deficient mice. $J$ Am Heart Assoc 6 e006297.

Wang L, Bao Y, Yang Y, Wu Y, Chen X, Si S, and Hong B (2010b) Discovery of antagonists for human scavenger receptor CD36 via an ELISA-like highthroughput screening assay. J Biomol Screen 15:239-250.

Wang L, Eftekhari P, Schachner D, Ignatova ID, Palme V, Schilcher N, Ladurner A Heiss EH, Stangl H, Dirsch VM, et al. (2018c) Novel interactomics approach identifies ABCA1 as direct target of evodiamine, which increases macrophage cholesterol efflux. Sci Rep 8:11061.

Wang L, Jia XJ, Jiang HJ, Du Y, Yang F, Si SY, and Hong B (2013) MicroRNAs 185, 96 , and 223 repress selective high-density lipoprotein cholesterol uptake through posttranscriptional inhibition. Mol Cell Biol 33:1956-1964.

Wang L, Ladurner A, Latkolik S, Schwaiger S, Linder T, Hošek J, Palme V, Schilcher N, Polanský O, Heiss EH, et al. (2016a) Leoligin, the major lignan from edelweiss (Leontopodium nivale subsp. alpinum), promotes cholesterol efflux from THP-1 macrophages. J Nat Prod 79:1651-1657.

Wang L, Palme V, Rotter S, Schilcher N, Cukaj M, Wang D, Ladurner A, Heiss EH Stangl H, Dirsch VM, et al. (2017d) Piperine inhibits ABCA1 degradation and promotes cholesterol efflux from THP-1-derived macrophages. Mol Nutr Food Res 61, doi: $10.1002 / \mathrm{mnfr} .201500960$.

Wang L, Palme V, Schilcher N, Ladurner A, Heiss EH, Stangl H, Bauer R, Dirsch VM, and Atanasov AG (2017e) The dietary constituent falcarindiol promotes cholesterol efflux from THP-1 macrophages by increasing ABCA1 gene transcription and protein stability. Front Pharmacol 8:596.

Wang L, Rotter S, Ladurner A, Heiss EH, Oberlies NH, Dirsch VM, and Atanasov AG (2015a) Silymarin constituents enhance ABCA1 expression in THP-1 macrophages Molecules 21:E55.

Wang L, Waltenberger B, Pferschy-Wenzig EM, Blunder M, Liu X, Malainer C, Blazevic T, Schwaiger S, Rollinger JM, Heiss EH, et al. (2014b) Natural product agonists of peroxisome proliferator-activated receptor gamma (PPAR $)$ : a review. Biochem Pharmacol 92:73-89.

Wang L, Wesemann S, Krenn L, Ladurner A, Heiss EH, Dirsch VM, and Atanasov AG (2017f) Erythrodiol, an olive oil constituent, increases the half-life of ABCA1 and enhances cholesterol efflux from THP-1-derived macrophages. Front Pharmacol 8:375.

Wang M and Briggs MR (2004) HDL: the metabolism, function, and therapeutic importance. Chem Rev 104:119-137.

Wang M, Li L, Liu R, Song Y, Zhang X, Niu W, Kumar AK, Guo Z, and Hu Z (2018d) Obesity-induced overexpression of miRNA-24 regulates cholesterol uptake and lipid metabolism by targeting SR-B1. Gene 668:196-203.

Wang N, Chen W, Linsel-Nitschke P, Martinez LO, Agerholm-Larsen B, Silver DL, and Tall AR (2003) A PEST sequence in ABCA1 regulates degradation by calpain protease and stabilization of ABCA1 by apoA-I. J Clin Invest 111:99-107.

Wang N, Lan D, Chen W, Matsuura F, and Tall AR (2004) ATP-binding cassette transporters G1 and G4 mediate cellular cholesterol efflux to high-density lipoproteins. Proc Natl Acad Sci USA 101:9774-9779.

Wang P, He LY, Shen GD, Li RL, and Yang JL (2017g) Inhibitory effects of Dioscin on atherosclerosis and foam cell formation in hyperlipidemia rats. Inflammopharmacology 25:633-642.

Wang Q, Zhang J, Li Y, Shi H, Wang H, Chen B, Wang F, Wang Z, Yang Z, and Wang L (2018e) Green tea polyphenol epigallocatechin-3-gallate increases atherosclerotic plaque stability in apolipoprotein E-deficient mice fed a high-fat diet. Kardiol Pol 76:1263-1270

Wang S and Smith JD (2014) ABCA1 and nascent HDL biogenesis. Biofactors 40: 547-554.

Wang S, Xie X, Lei T, Zhang K, Lai B, Zhang Z, Guan Y, Mao G, Xiao L, and Wang N (2017h) Statins attenuate activation of the NLRP3 inflammasome by oxidized LDI or TNF $\alpha$ in vascular endothelial cells through a PXR-dependent mechanism. Mol Pharmacol 92:256-264.

Wang S, Zhang X, Liu M, Luan H, Ji Y, Guo P, and Wu C (2015b) Chrysin inhibits foam cell formation through promoting cholesterol efflux from RAW264.7 macrophages. Pharm Biol 53:1481-1487.

Wang W, Zhang ZZ, Wu Y, Wang RQ, Chen JW, Chen J, Zhang Y, Chen YJ, Geng M, $\mathrm{Xu} \mathrm{ZD}$, et al. (2018f) (-)-Epigallocatechin-3-gallate ameliorates atherosclerosis and modulates hepatic lipid metabolic gene expression in apolipoprotein $\mathrm{E}$ knockout mice: involvement of TTC39B. Front Pharmacol 9:195.

Wang X, Collins HL, Ranalletta M, Fuki IV, Billheimer JT, Rothblat GH, Tall AR, and Rader DJ (2007a) Macrophage ABCA1 and ABCG1, but not SR-BI, promote macrophage reverse cholesterol transport in vivo. J Clin Invest 117:2216-2224.

Wang X, Mu H, Chai H, Liao D, Yao Q, and Chen C (2007b) Human immunodeficiency virus protease inhibitor ritonavir inhibits cholesterol efflux from human macrophage-derived foam cells. Am J Pathol 171:304-314.

Wang XQ, Wan HQ, Wei XJ, Zhang Y, and Qu P (2016b) CLI-095 decreases ath erosclerosis by modulating foam cell formation in apolipoprotein E-deficient mice. Mol Med Rep 14:49-56.

Wang Y, Dubland JA, Allahverdian S, Asonye E, Sahin B, Jaw JE, Sin DD, Seidman MA, Leeper NJ, and Francis GA (2019b) Smooth muscle cells contribute the majority of foam cells in ApoE (apolipoprotein E)-deficient mouse atherosclerosis. Arterioscler Thromb Vasc Biol 39:876-887.

Wang Y, Rao L, Zhou B, Chen Y, Peng Y, Song Y, and Zhang L (2010c) The G501C polymorphism of the oxidized low-density lipoprotein-receptor 1 gene is associated with acute coronary syndrome in the Han Chinese population. DNA Cell Biol 29 201-205.

Wang Y, Wang YS, Song SL, Liang H, and Ji AG (2016c) Icariin inhibits atherosclerosis progress in Apoe null mice by downregulating CX3CR1 in macrophage. Biochem Biophys Res Commun 470:845-850.

Wang Y, Zhang Y, Wang X, Liu Y, and Xia M (2012b) Cyanidin-3-O- $\beta$-glucoside induces oxysterol efflux from endothelial cells: role of liver $\mathrm{X}$ receptor alpha. Ath erosclerosis 223:299-305.

Wang Y, Zhang Y, Wang X, Liu Y, and Xia M (2012c) Supplementation with cyanidin3 -O- $\beta$-glucoside protects against hypercholesterolemia-mediated endothelial dysfunction and attenuates atherosclerosis in apolipoprotein E-deficient mice. J Nutr 142:1033-1037.

Wang YC, Lee AS, Lu LS, Ke LY, Chen WY, Dong JW, Lu J, Chen Z, Chu CS, Chan $\mathrm{HC}$, et al. (2018g) Human electronegative LDL induces mitochondrial dysfunction and premature senescence of vascular cells in vivo. Aging Cell 17:e12792.

Wang YF, Yang XF, Cheng B, Mei CL, Li QX, Xiao H, Zeng QT, Liao YH, and Liu K (2010d) Protective effect of astragalus polysaccharides on ATP binding cassette transporter A1 in THP-1 derived foam cells exposed to tumor necrosis factor-alpha. Phytother Res 24:393-398.

Wang YH, Chen YF, Chen SR, Chen X, Chen JW, Shen XY, Mou YG, and Liu PQ (2010e) Aspirin increases apolipoprotein-A-I-mediated cholesterol efflux via enhancing expression of ATP-binding cassette transporter A1. Pharmacology 86: 320-326.

Wang YK, Hong YJ, and Huang ZQ (2005) Protective effects of silybin on human umbilical vein endothelial cell injury induced by $\mathrm{H} 2 \mathrm{O} 2$ in vitro. Vascul Pharmacol 43:198-206.

Wang YQ, Dai M, Zhong JC, and Yin DK (2012d) Paeonol inhibits oxidized low density lipoprotein-induced monocyte adhesion to vascular endothelial cells by inhibiting the mitogen activated protein kinase pathway. Biol Pharm Bull 35: $767-772$.

Wang YT, Wang YH, Ma YT, Fu ZY, Yang YN, Ma X, Li XM, Adi D, Liu F, and Chen $\mathrm{BD}$ (2017i) ACAT-1 gene polymorphism is associated with increased susceptibility to coronary artery disease in Chinese Han population: a case-control study. Oncotarget 8:89055-89063.

Wei L, Deng W, Cheng Z, Guo H, Wang S, Zhang X, He Y, and Tang Q (2016) Effects of hesperetin on platelet-derived growth factor-BB-induced pulmonary artery smooth muscle cell proliferation. Mol Med Rep 13:955-960.

Weibel GL, Hayes S, Wilson A, Phillips MC, Billheimer J, Rader DJ, and Rothblat GH (2011) Novel in vivo method for measuring cholesterol mass flux in peripheral macrophages. Arterioscler Thromb Vasc Biol 31:2865-2871.

Weinreich M and Frishman WH (2014) Antihyperlipidemic therapies targeting PCSK9. Cardiol Rev 22:140-146.

Weintraub $\mathrm{H}$ (2013) Update on marine omega-3 fatty acids: management of dyslipidemia and current omega-3 treatment options. Atherosclerosis 230:381-389.

Weng S, Sprague JE, Oh J, Riek AE, Chin K, Garcia M, and Bernal-Mizrachi C (2013) Vitamin D deficiency induces high blood pressure and accelerates atherosclerosis in mice. PLoS One 8:e54625.

Westerterp M, Fotakis P, Ouimet M, Bochem AE, Zhang H, Molusky MM, Wang W, Abramowicz S, la Bastide-van Gemert S, Wang N, et al. (2018) Cholesterol efflux pathways suppress inflammasome activation, NETosis, and atherogenesis. Circulation 138:898-912.

White SJ, Sala-Newby GB, and Newby AC (2011) Overexpression of scavenger receptor LOX-1 in endothelial cells promotes atherogenesis in the ApoE(-/-) mouse model. Cardiovasc Patho 20:369-373.

Whitman SC, Rateri DL, Szilvassy SJ, Cornicelli JA, and Daugherty A (2002) Macrophage-specific expression of class A scavenger receptors in LDL receptor(-/-) mice decreases atherosclerosis and changes spleen morphology. J Lipid Res 43 : $1201-1208$

Williams DL, Connelly MA, Temel RE, Swarnakar S, Phillips MC, de la Llera-Moya M, and Rothblat GH (1999) Scavenger receptor BI and cholesterol trafficking. Curr Opin Lipidol 10:329-339.

Willner EL, Tow B, Buhman KK, Wilson M, Sanan DA, Rudel LL, and Farese RV Jr (2003) Deficiency of acyl CoA:cholesterol acyltransferase 2 prevents atherosclerosis in apolipoprotein E-deficient mice. Proc Natl Acad Sci USA 100:1262-1267.

Wool GD, Reardon CA, and Getz GS (2008) Apolipoprotein A-I mimetic peptide helix number and helix linker influence potentially anti-atherogenic properties. J Lipid Res 49:1268-1283.

Wu C, Chen R, Liu M, Liu D, Li X, Wang S, Niu S, Guo P, and Lin W (2015a) Spiromastixones inhibit foam cell formation via regulation of cholesterol efflux and uptake in RAW264.7 macrophages. Mar Drugs 13:6352-6365.

Wu C, Luan H, Zhang X, Wang S, Zhang X, Sun X, and Guo P (2014) Chlorogenic acid protects against atherosclerosis in ApoE-/- mice and promotes cholesterol efflux from RAW264.7 macrophages. PLoS One 9:e95452.

Wu H and Ballantyne CM (2017) Dyslipidaemia: PCSK9 inhibitors and foamy monocytes in familial hypercholesterolaemia. Nat Rev Cardiol 14:385-386.

Wu M, Liu M, Guo G, Zhang W, and Liu L (2015b) Polydatin inhibits formation of macrophage-derived foam cells. Evid Based Complement Alternat Med 2015: 729017.

Wu S, Xu H, Peng J, Wang C, Jin Y, Liu K, Sun H, and Qin J (2015c) Potent antiinflammatory effect of dioscin mediated by suppression of TNF- $\alpha$-induced VCAM-1, ICAM-1and EL expression via the NF-кB pathway. Biochimie 110:62-72.

Wu WH, Wang SH, Kuan II, Kao YS, Wu PJ, Liang CJ, Chien HF, Kao CH, Huang CJ, and Chen YL (2010) Sesamin attenuates intercellular cell adhesion molecule-1 expression in vitro in TNF-alpha-treated human aortic endothelial cells and in vivo in apolipoprotein-E-deficient mice. Mol Nutr Food Res 54:1340-1350.

Wu XL, Liou CJ, Li ZY, Lai XY, Fang LW, and Huang WC (2015d) Sesamol suppresses the inflammatory response by inhibiting NF-kB/MAPK activation and 
upregulating AMP kinase signaling in RAW 264.7 macrophages. Inflamm Res 64 $577-588$

Wuttge DM, Romert A, Eriksson U, Törmä H, Hansson GK, and Sirsjö A (2001) Induction of CD36 by all-trans retinoic acid: retinoic acid receptor signaling in the pathogenesis of atherosclerosis. FASEB J 15:1221-1223.

Xia M, Hou M, Zhu H, Ma J, Tang Z, Wang Q, Li Y, Chi D, Yu X, Zhao T, et al. (2005) Anthocyanins induce cholesterol efflux from mouse peritoneal macrophages: the role of the peroxisome proliferator-activated receptor gamma-liver $\mathrm{X}$ receptor alpha-ABCA1 pathway. J Biol Chem 280:36792-36801.

Xia X, Ling W, Ma J, Xia M, Hou M, Wang Q, Zhu H, and Tang Z (2006) An anthocyanin-rich extract from black rice enhances atherosclerotic plaque stabilization in apolipoprotein E-deficient mice. J Nutr 136:2220-2225.

Xiao HB, Sui GG, and Lu XY (2017) Icariin improves eNOS/NO pathway to prohibit the atherogenesis of apolipoprotein E-null mice. Can J Physiol Pharmacol 95: $625-633$.

Xie X, Wang S, Xiao L, Zhang J, Wang J, Liu J, Shen X, He D, Zheng X, and Zhai Y (2011) DBZ blocks LPS-induced monocyte activation and foam cell formation via inhibiting nuclear factor-кB. Cell Physiol Biochem 28:649-662.

Xu J, Hu G, Lu M, Xiong Y, Li Q, Chang CC, Song B, Chang T, and Li B (2013a) MiR-9 reduces human acyl-coenzyme A:cholesterol acyltransferase-1 to decrease THP-1 macrophage-derived foam cell formation. Acta Biochim Biophys Sin (Shanghai) 45:953-962

Xu M, Zhou H, Tan KC, Guo R, Shiu SW, and Wong Y (2009) ABCG1 mediated oxidized LDL-derived oxysterol efflux from macrophages. Biochem Biophys Res Commun 390:1349-1354.

Xu S (2017) Transcriptome profiling in systems vascular medicine. Front Pharmacol 8:563.

Xu S, Huang Y, Xie Y, Lan T, Le K, Chen J, Chen S, Gao S, Xu X, Shen X, et al. (2010) Evaluation of foam cell formation in cultured macrophages: an improved method with Oil Red O staining and DiI-oxLDL uptake. Cytotechnology 62:473-481.

Xu S, Kamato D, Little PJ, Nakagawa S, Pelisek J, and Jin ZG (2019) Targeting epigenetics and non-coding RNAs in atherosclerosis: from mechanisms to therapeutics. Pharmacol Ther 196:15-43.

Xu S and Liu P (2013) Tanshinone II-A: new perspectives for old remedies. Expert Opin Ther Pat 23:149-153.

Xu S, Liu Z, and Liu P (2013b) HDL cholesterol in cardiovascular diseases: the good, the bad, and the ugly? Int $J$ Cardiol 168:3157-3159.

Xu S, Ogura S, Chen J, Little PJ, Moss J, and Liu P (2013c) LOX-1 in atherosclerosis: biological functions and pharmacological modifiers. Cell Mol Life Sci $\mathbf{7 0}$ 2859-2872.

Xu S, Pelisek J, and Jin ZG (2018) Atherosclerosis is an epigenetic disease. Trends Endocrinol Metab 29:739-742.

Xu X, Li Q, Pang L, Huang G, Huang J, Shi M, Sun X, and Wang Y (2013d) Arctigenin promotes cholesterol efflux from THP-1 macrophages through PPAR- $\gamma / L X R-\alpha$ signaling pathway. Biochem Biophys Res Commun 441:321-326.

Xu X, Pan J, and Zhou X (2014a) Amelioration of lipid profile and level of antioxidant activities by epigallocatechin-gallate in a rat model of atherogenesis. Heart Lung Circ 23:1194-1201.

Xu Y, Liu Q, Xu Y, Liu C, Wang X, He X, Zhu N, Liu J, Wu Y, Li Y, et al. (2014b) Rutaecarpine suppresses atherosclerosis in ApoE-/- mice through upregulating ABCA1 and SR-BI within RCT. J Lipid Res 55:1634-1647.

Yagyu H, Kitamine T, Osuga J, Tozawa R, Chen Z, Kaji Y, Oka T, Perrey S, Tamura Y, Ohashi K, et al. (2000) Absence of ACAT-1 attenuates atherosclerosis but causes dry eye and cutaneous xanthomatosis in mice with congenital hyperlipidemia. $J$ Biol Chem 275:21324-21330.

Yamamoto Y and Gaynor RB (2001) Therapeutic potential of inhibition of the NFkappaB pathway in the treatment of inflammation and cancer. $J$ Clin Invest 107: 135-142.

Yang H, Yan L, Qian P, Duan H, Wu J, Li B, and Wang S (2015) Icariin inhibits foam cell formation by down-regulating the expression of CD36 and up-regulating the expression of SR-BI. J Cell Biochem 116:580-588.

Yang HL, Chen SC, Senthil Kumar KJ, Yu KN, Lee Chao PD, Tsai SY, Hou YC, and Hseu YC (2012) Antioxidant and anti-inflammatory potential of hesperetin metabolites obtained from hesperetin-administered rat serum: an ex vivo approach. J Agric Food Chem 60:522-532.

Yang RX, Huang SY, Yan FF, Lu XT, Xing YF, Liu Y, Liu YF, and Zhao YX (2010) Danshensu protects vascular endothelia in a rat model of hyperhomocysteinemia. Acta Pharmacol Sin 31:1395-1400.

Yang S, Ye ZM, Chen S, Luo XY, Chen SL, Mao L, Li Y, Jin H, Yu C, Xiang FX, et al (2018a) MicroRNA-23a-5p promotes atherosclerotic plaque progression and vulnerability by repressing ATP-binding cassette transporter A1/G1 in macrophages. J Mol Cell Cardiol 123:139-149.

Yang TL, Lin FY, Chen YH, Chiu JJ, Shiao MS, Tsai CS, Lin SJ, and Chen YL (2011) Salvianolic acid B inhibits low-density lipoprotein oxidation and neointimal hyperplasia in endothelium-denuded hypercholesterolaemic rabbits. J Sci Food Agric 91:134-141.

Yang X, Yin M, Yu L, Lu M, Wang H, Tang F, and Zhang Y (2016) Simvastatin inhibited oxLDL-induced proatherogenic effects through calpain-1-PPAR $\gamma$-CD36 pathway. Can J Physiol Pharmacol 94:1336-1343.

Yang Y, Jiang W, Wang L, Zhang ZB, Si SY, and Hong B (2009) Characterization of the isoflavone pratensein as a novel transcriptional up-regulator of scavenger receptor class B type I in HepG2 cells. Biol Pharm Bull 32:1289-1294.

Yang Y, Li X, Peng L, An L, Sun N, Hu X, Zhou P, Xu Y, Li P, and Chen J (2018b) Tanshindiol C inhibits oxidized low-density lipoprotein induced macrophage foam cell formation via a peroxiredoxin 1 dependent pathway. Biochim Biophys Acta Mol Basis Dis 1864:882-890.

Yang Y, Zhang Z, Jiang W, Gao L, Zhao G, Zheng Z, Wang M, Si S, and Hong B (2007) Identification of novel human high-density lipoprotein receptor Up-regulators using a cell-based high-throughput screening assay. J Biomol Screen 12:211-219.

Yasuhara M, Saito K, Kubota H, Ohmizu H, and Suzuki T (1997) Inhibitory effect of a new ureidophenol derivative T-2591 on LDL oxidation and ACAT activity. Biol Pharm Bull 20:1056-1060.
Yeaman SJ (2004) Hormone-sensitive lipase $==$ new roles for an old enzyme. Biochem $J$ 379:11-22.

Yin K, You Y, Swier V, Tang L, Radwan MM, Pandya AN, and Agrawal DK (2015) Vitamin D protects against atherosclerosis via regulation of cholesterol efflux and macrophage polarization in hypercholesterolemic swine. Arterioscler Thromb Vasc Biol 35:2432-2442.

Yin YW, Liao SQ, Zhang MJ, Liu Y, Li BH, Zhou Y, Chen L, Gao CY, Li JC, and Zhang LL (2014) TLR4-mediated inflammation promotes foam cell formation of vascular smooth muscle cell by upregulating ACAT1 expression. Cell Death Dis 5:e1574.

Ying Z, Kherada N, Kampfrath T, Mihai G, Simonetti O, Desikan R, Selvendiran K, Sun Q, Ziouzenkova O, Parthasarathy S, et al. (2011) A modified sesamol derivative inhibits progression of atherosclerosis. Arterioscler Thromb Vasc Biol 31 $536-542$.

Yokoyama S (2006) Assembly of high-density lipoprotein. Arterioscler Thromb Vasc Biol 26:20-27.

Yokoyama S, Arakawa R, Wu CA, Iwamoto N, Lu R, Tsujita M, and Abe-Dohmae S (2012) Calpain-mediated ABCA1 degradation: post-translational regulation of ABCA1 for HDL biogenesis. Biochim Biophys Acta 1821:547-551.

Yoshida H, Yanai H, Ito K, Tomono Y, Koikeda T, Tsukahara H, and Tada N (2010) Administration of natural astaxanthin increases serum HDL-cholesterol and adiponectin in subjects with mild hyperlipidemia. Atherosclerosis 209:520-523.

Yoshimoto R, Fujita Y, Kakino A, Iwamoto S, Takaya T, and Sawamura T (2011) The discovery of LOX-1, its ligands and clinical significance. Cardiovasc Drugs Ther $\mathbf{2 5}$ 379-391.

Yoshinaka Y, Shibata H, Kobayashi H, Kuriyama H, Shibuya K, Tanabe S, Watanabe T, and Miyazaki A (2010) A selective ACAT-1 inhibitor, K-604, stimulates collagen production in cultured smooth muscle cells and alters plaque phenotype in apolipoprotein E-knockout mice. Atherosclerosis 213:85-91.

Yu P, Xiong T, Tenedero CB, Lebeau P, Ni R, MacDonald ME, Gross PL, Austin RC, and Trigatti BL (2018) Rosuvastatin reduces aortic sinus and coronary artery atherosclerosis in SR-B1 (scavenger receptor class B type 1)/ApoE (Apolipoprotein E) double knockout mice independently of plasma cholesterol lowering. Arterioscler Thromb Vasc Biol 38:26-39.

Yu R, Lv Y, Wang J, Pan N, Zhang R, Wang X, Yu H, Tan L, Zhao Y, and Li B (2016) Baicalin promotes cholesterol efflux by regulating the expression of SR-BI in macrophages. Exp Ther Med 12:4113-4120.

Yu XH, Fu YC, Zhang DW, Yin K, and Tang CK (2013) Foam cells in atherosclerosis. Clin Chim Acta 424:245-252.

Yuan X, Chen J, and Dai M (2016) Paeonol promotes microRNA-126 expression to inhibit monocyte adhesion to ox-LDL-injured vascular endothelial cells and block the activation of the PI3K/Akt/NF-kB pathway. Int J Mol Med 38:1871-1878.

Yuan Z, Liao Y, Tian G, Li H, Jia Y, Zhang H, Tan Z, Li X, Deng W, Liu K, et al. (2011) Panax notoginseng saponins inhibit Zymosan A induced atherosclerosis by suppressing integrin expression, FAK activation and NF-кB translocation. $J$ Ethnopharmacol 138:150-155.

Yue J, Li B, Jing Q, and Guan Q (2015) Salvianolic acid B accelerated ABCA1dependent cholesterol efflux by targeting PPAR- $\gamma$ and LXR $\alpha$. Biochem Biophys Res Commun 462:233-238.

Yuhanna IS, Zhu Y, Cox BE, Hahner LD, Osborne-Lawrence S, Lu P, Marcel YL, Anderson RG, Mendelsohn ME, Hobbs HH, et al. (2001) High-density lipoprotein binding to scavenger receptor-BI activates endothelial nitric oxide synthase. Nat Med 7:853-857.

Yvan-Charvet L, Matsuura F, Wang N, Bamberger MJ, Nguyen T, Rinninger F, Jiang XC, Shear CL, and Tall AR (2007a) Inhibition of cholesteryl ester transfer protein by torcetrapib modestly increases macrophage cholesterol efflux to HDL. Arterioscler Thromb Vasc Biol 27:1132-1138.

Yvan-Charvet L, Pagler TA, Seimon TA, Thorp E, Welch CL, Witztum JL, Tabas I, and Tall AR (2010a) ABCA1 and ABCG1 protect against oxidative stress-induced macrophage apoptosis during efferocytosis. Circ Res 106:1861-1869.

Yvan-Charvet L, Pagler TA, Wang N, Senokuchi T, Brundert M, Li H, Rinninger F, and Tall AR (2008) SR-BI inhibits ABCG1-stimulated net cholesterol efflux from cells to plasma HDL. J Lipid Res 49:107-114

Yvan-Charvet L, Ranalletta M, Wang N, Han S, Terasaka N, Li R, Welch C, and Tall $\mathrm{AR}$ (2007b) Combined deficiency of ABCA1 and ABCG1 promotes foam cell accumulation and accelerates atherosclerosis in mice. J Clin Invest 117:3900-3908.

Yvan-Charvet L, Wang N, and Tall AR (2010b) Role of HDL, ABCA1, and ABCG1 transporters in cholesterol efflux and immune responses. Arterioscler Thromb Vasc Biol 30:139-143.

Zani IA, Stephen SL, Mughal NA, Russell D, Homer-Vanniasinkam S, Wheatcroft SB, and Ponnambalam S (2015) Scavenger receptor structure and function in health and disease. Cells 4:178-201.

Zannis VI, Chroni A, and Krieger M (2006) Role of apoA-I, ABCA1, LCAT, and SR-BI in the biogenesis of HDL. J Mol Med (Berl) 84:276-294.

Zarubica A, Trompier D, and Chimini G (2007) ABCA1, from pathology to membrane function. Pflugers Arch 453:569-579.

Zeboudj L, Giraud A, Guyonnet L, Zhang Y, Laurans L, Esposito B, Vilar J, Chipont A, Papac-Milicevic N, Binder CJ, et al. (2018) Selective EGFR (epidermal growth factor receptor) deletion in myeloid cells limits atherosclerosis-brief report. Arterioscler Thromb Vasc Biol 38:114-119.

Zechner R, Moser R, Newman TC, Fried SK, and Breslow JL (1991) Apolipoprotein E gene expression in mouse 3T3-L1 adipocytes and human adipose tissue and its regulation by differentiation and lipid content. J Biol Chem 266:10583-10588.

Zhang H, Zhai Z, Zhou H, Li Y, Li X, Lin Y, Li W, Shi Y, and Zhou MS (2015) Puerarin inhibits oxLDL-induced macrophage activation and foam cell formation in human THP1 macrophage. BioMed Res Int 2015:403616.

Zhang J, Cai S, Peterson BR, Kris-Etherton PM, and Heuvel JP (2011) Development of a cell-based, high-throughput screening assay for cholesterol efflux using a fluorescent mimic of cholesterol. Assay Drug Dev Technol 9:136-146.

Zhang J, Nie S, Martinez-Zaguilan R, Sennoune SR, and Wang S (2016a) Formulation, characteristics and antiatherogenic bioactivities of CD36-targeted epigallocatechin gallate (EGCG)-loaded nanoparticles. J Nutr Biochem 30:14-23. 
Zhang J, Nie S, and Wang S (2013) Nanoencapsulation enhances epigallocatechin-3gallate stability and its antiatherogenic bioactivities in macrophages. J Agric Food Chem 61:9200-9209.

Zhang L, Issa Bhaloo S, Chen T, Zhou B, and Xu Q (2018) Role of resident stem cells in vessel formation and arteriosclerosis. Circ Res 122:1608-1624.

Zhang L, Liu HJ, Li TJ, Yang Y, Guo XL, Wu MC, Rui YC, and Wei LX (2008) Lentiviral vector-mediated siRNA knockdown of SR-PSOX inhibits foam cell formation in vitro. Acta Pharmacol Sin 29:847-852.

Zhang M, Wu JF, Chen WJ, Tang SL, Mo ZC, Tang YY, Li Y, Wang JL, Liu XY, Peng $\mathrm{J}$, et al. (2014) MicroRNA-27a/b regulates cellular cholesterol efflux, influx and esterification/hydrolysis in THP-1 macrophages. Atherosclerosis 234:54-64.

Zhang MJ, Zhou Y, Chen L, Wang X, Pi Y, Long CY, Sun MJ, Chen X, Gao CY, Li JC, et al. (2016b) Impaired SIRT1 promotes the migration of vascular smooth muscle cell-derived foam cells. Histochem Cell Biol 146:33-43.

Zhang W, Yancey PG, Su YR, Babaev VR, Zhang Y, Fazio S, and Linton MF (2003a) Inactivation of macrophage scavenger receptor class B type I promotes atherosclerotic lesion development in apolipoprotein E-deficient mice. Circulation 108: $2258-2263$

Zhang X, Moor AN, Merkler KA, Liu Q, and McLean MP (2007) Regulation of alternative splicing of liver scavenger receptor class $\mathrm{B}$ gene by estrogen and the involved regulatory splicing factors. Endocrinology 148:5295-5304.

Zhang Y, Zanotti I, Reilly MP, Glick JM, Rothblat GH, and Rader DJ (2003b) Overexpression of apolipoprotein A-I promotes reverse transport of cholesterol from macrophages to feces in vivo. Circulation 108:661-663.

Zhang Z, Zhang D, Du B, and Chen Z (2017) Hyperoside inhibits the effects induced by oxidized low-density lipoprotein in vascular smooth muscle cells via oxLDLLOX-1-ERK pathway. Mol Cell Biochem 433:169-176.

Zhao B, Song J, Chow WN, St Clair RW, Rudel LL, and Ghosh S (2007) Macrophagespecific transgenic expression of cholesteryl ester hydrolase significantly reduces atherosclerosis and lesion necrosis in Ldlr mice. J Clin Invest 117:2983-2992.

Zhao GJ, Tang SL, Lv YC, Ouyang XP, He PP, Yao F, Chen WJ, Lu Q, Tang YY, Zhang M, et al. (2013a) Antagonism of betulinic acid on LPS-mediated inhibition of ABCA1 and cholesterol efflux through inhibiting nuclear factor-kappaB signaling pathway and miR-33 expression. PLoS One 8:e74782.

Zhao JF, Ching LC, Huang YC, Chen CY, Chiang AN, Kou YR, Shyue SK, and Lee TS (2012) Molecular mechanism of curcumin on the suppression of cholesterol accumulation in macrophage foam cells and atherosclerosis. Mol Nutr Food Res $\mathbf{5 6}$ 691-701.

Zhao JF, Jim Leu SJ, Shyue SK, Su KH, Wei J, and Lee TS (2013b) Novel effect of paeonol on the formation of foam cells: promotion of LXR $\alpha$-ABCA1-dependent cholesterol efflux in macrophages. Am J Chin Med 41:1079-1096.

Zhao S, Li J, Wang L, and Wu X (2016) Pomegranate peel polyphenols inhibit lipid accumulation and enhance cholesterol efflux in raw264.7 macrophages. Food Funct 7:3201-3210.
Zhao W, Haller V, and Ritsch A (2015) The polyphenol PGG enhances expression of SR-BI and ABCA1 in J774 and THP-1 macrophages. Atherosclerosis 242:611-617. Zhao W, Ma G, and Chen X (2014) Lipopolysaccharide induced LOX-1 expression via TLR4/MyD88/ROS activated p38MAPK-NF-кB pathway. Vascul Pharmacol 63: $162-172$.

Zhao XY, Qiao GF, Li BX, Chai LM, Li Z, Lu YJ, and Yang BF (2009) Hypoglycaemic and hypolipidaemic effects of emodin and its effect on L-type calcium channels in dyslipidaemic-diabetic rats. Clin Exp Pharmacol Physiol 36:29-34.

Zhao Z and Moghadasian MH (2008) Chemistry, natural sources, dietary intake and pharmacokinetic properties of ferulic acid: a review. Food Chem 109:691-702.

Zhao ZZ, Wang Z, Li GH, Wang R, Tan JM, Cao X, Suo R, and Jiang ZS (2011) Hydrogen sulfide inhibits macrophage-derived foam cell formation. Exp Biol Med (Maywood) 236:169-176.

Zhong X and Liu H (2018) Honokiol attenuates diet-induced non-alcoholic steatohepatitis by regulating macrophage polarization through activating peroxisome proliferator-activated receptor $\gamma$. J Gastroenterol Hepatol 33:524-532.

Zhou JH, Pan ZY, Zhang YF, Cui WY, Long CL, and Wang H (2014) Stimulation of endothelial non-neuronal muscarinic receptor attenuates the progression of atherosclerosis via inhibiting endothelial cells activation. Zhongguo Ying Yong Sheng Li Xue Za Zhi 30:549-559.

Zhou M, Xu H, Pan L, Wen J, Guo Y, and Chen K (2008) Emodin promotes atherosclerotic plaque stability in fat-fed apolipoprotein E-deficient mice. Tohoku J Exp Med 215:61-69.

Zhou W, Lin J, Chen H, Wang J, Liu Y, and Xia M (2015) Retinoic acid induces macrophage cholesterol efflux and inhibits atherosclerotic plaque formation in apoE-deficient mice. Br J Nutr 114:509-518.

Zhou Y, Chen R, Liu D, Wu C, Guo P, and Lin W (2017) Asperlin inhibits LPS-evoked foam cell formation and prevents atherosclerosis in $\mathrm{ApoE}^{-1-}$ mice. Mar Drugs 15: E358.

Zhu L, Zhang D, Zhu H, Zhu J, Weng S, Dong L, Liu T, Hu Y, and Shen X (2018) Berberine treatment increases Akkermansia in the gut and improves high-fat dietinduced atherosclerosis in Apoe ${ }^{-/-}$mice. Atherosclerosis 268:117-126.

Zhu S and Liu JH (2015) Zerumbone, a natural cyclic sesquiterpene, promotes ABCA1-dependent cholesterol efflux from human THP-1 macrophages. Pharmacology 95:258-263.

Zhu Y, Ling W, Guo H, Song F, Ye Q, Zou T, Li D, Zhang Y, Li G, Xiao Y, et al. (2013) Anti-inflammatory effect of purified dietary anthocyanin in adults with hypercholesterolemia: a randomized controlled trial. Nutr Metab Cardiovasc Dis 23: 843-849.

Zhu ZD, Jia JQ, Zhang X, Wang YJ, and Wang DH (2010) Asymmetric dimethylarginine upregulates the expression of ACAT-1 in THP-1 macrophage-derived foam cells. Nan Fang Yi Ke Da Xue Xue Bao 30:2613-2618.

Zolberg Relevy N, Bechor S, Harari A, Ben-Amotz A, Kamari Y, Harats D, and Shaish A (2015) The inhibition of macrophage foam cell formation by 9 -cis $\beta$-carotene is driven by BCMO1 activity. PLoS One 10:e0115272. 